\title{
Relações min-max em otimização combinatória
}

\author{
Marcel Kenji de Carli Silva \\ DISSERTAÇÃO APRESENTADA \\ $\mathrm{AO}$ \\ INSTITUTO DE MATEMÁTICA E ESTATÍSTICA \\ DA \\ UNIVERSIDADE DE SÃO PAULO \\ PARA \\ OBTENÇÃO DO TÍTULO DE MESTRE \\ EM \\ CIÊNCIAS
}

Área de Concentração: Ciência da Computação

Orientador: Profa. Dra. Yoshiko Wakabayashi

Durante a elaboração deste trabalho o autor recebeu auxílio financeiro da FAPESP através do processo 04/11338-3

São Paulo, maio de 2007 



\section{Relações min-max em otimização combinatória}

Este exemplar corresponde à redação final da dissertação devidamente corrigida e defendida por Marcel K. de Carli Silva e aprovada pela comissão julgadora.

São Paulo, 3 de maio de 2007

Banca examinadora:

Profa. Dra. Yoshiko Wakabayashi (presidente) IME-USP

Prof. Dr. Paulo Feofiloff IME-USP

Prof. Dr. Cláudio Leonardo Lucchesi IC-UNICAMP 



\title{
Agradecimentos
}

\author{
Agradeço
}

à Yoshiko pela paciência e excelente orientação;

aos membros da banca examinadora pela leitura cuidadosa desta dissertação;

aos meus pais, pelo carinho ao longo de todos esses anos;

à Cristiane, por estar presente em todos os momentos;

aos amigos do BCC 2001: Cardonha, Hashimoto, Ju, Ellen e Domingos;

aos professores Yoshi e Cris, pelo constante apoio;

ao Carlinhos e ao Pil, pela diversão nas maratonas de programação e pela várzea;

aos colegas do BCC 2000: Daniel Morgato e Fernando Mario;

ao pessoal do VIP Lab: Antonio Carlos, Chicão, Domingos Soares, Fabrécio, Goca, Lobato, Luna, Marcio e Ricardo.

Obrigado a todos! 



\section{Resumo}

Relações min-max são objetos centrais em otimização combinatória. Elas basicamente afirmam que, numa dada estrutura, o valor ótimo de um certo problema de minimização é igual ao valor ótimo de um outro problema de maximização. Relações desse tipo fornecem boas caracterizações e descrições poliédricas para diversos problemas importantes, além de geralmente virem acompanhadas de algoritmos eficientes para os problemas em questão. Muitas vezes, tais algoritmos eficientes são obtidos naturalmente das provas construtivas dessas relações; mesmo quando isso não ocorre, essas relações revelam o suficiente sobre a estrutura combinatória dos problemas, levando ao desenvolvimento de algoritmos eficientes.

O foco principal desta dissertação é o estudo dessas relações em grafos. Nossa ênfase é sobre grafos orientados. Apresentamos o poderoso arcabouço poliédrico de Edmonds e Giles envolvendo fluxos submodulares, bem como o algoritmo de Frank para um caso especial desse arcabouço: o teorema de Lucchesi-Younger. Derivamos também diversas relações min-max sobre o empacotamento de conectores, desde o teorema de ramificações disjuntas de Edmonds até o teorema de junções disjuntas de Feofiloff-Younger e Schrijver.

Apresentamos também uma resenha completa sobre as conjecturas de Woodall e sua versão capacitada, conhecida como conjectura de Edmonds-Giles.

Derivamos ainda algumas relações min-max clássicas sobre emparelhamentos, $T$-junções e $S$-caminhos. Para tanto, usamos um teorema de Frank, Tardos e Sebő e um arcabouço bastante geral devido a Chudnovsky, Geelen, Gerards, Goddyn, Lohman e Seymour.

Ao longo do texto, ilustramos vários aspectos recorrentes, como o uso de ferramentas da combinatória poliédrica, a técnica do descruzamento, o uso de funções submodulares, matróides e propriedades de troca, bem como alguns resultados envolvendo subestruturas proibidas. 



\section{Abstract}

Min-max relations are central objects in combinatorial optimization. They basically state that, in a given structure, the optimum value of a certain minimization problem equals the optimum value of a different, maximization problem. Relations of this kind provide good characterizations and polyhedral descriptions to several important problems and, moreover, they often come with efficient algorithms for the corresponding problems. Usually, such efficient algorithms are obtained naturally from the constructive proofs involved; even when that is not the case, these relations reveal enough of the combinatorial structure of the problem, leading to the development of efficient algorithms.

The main focus of this dissertation is the study of these relations in graphs. Our emphasis is on directed graphs. We present Edmonds and Giles' powerful polyhedral framework concerning submodular flows, as well as Frank's algorithm for a special case of this framework: the Lucchesi-Younger Theorem. We also derive several min-max relations about packing connectors, starting with Edmonds' Disjoint Branchings Theorem and ending with FeofiloffYounger and Schrijver's Disjoint Dijoins Theorem.

We further derive some classical min-max relations on matchings, $T$-joins and $S$-paths. To this end, we use a theorem due to Frank, Tardos, and Sebö and a general framework due to Chudnovsky, Geelen, Gerards, Goddyn, Lohman, and Seymour.

Throughout the text, we illustrate several recurrent themes, such as the use of tools from polyhedral combinatorics, the uncrossing technique, the use of submodular functions, matroids and exchange properties, as well as some results involving forbidden substructures. 



\section{Sumário}

Agradecimentos $\quad$ V

Resumo vii

$\begin{array}{ll}\text { Abstract } & \text { ix }\end{array}$

$\begin{array}{ll}\text { Introdução } & 1\end{array}$

1 Preliminares $\quad 9$

1.1 Preliminares gerais . . . . . . . . . . . . . . . . . . . 10

1.2 Grafos . . . . . . . . . . . . . . . . . . . . . . 11

1.3 Grafos orientados . . . . . . . . . . . . . . . . . . 14

1.4 Hipergrafos . . . . . . . . . . . . . . . . . . . . . . . . . . . . . . . . . . .

1.5 Programação linear e inteira . . . . . . . . . . . . . . . . . 17

1.6 Matróides . . . . . . . . . . . . . . . . . . . . 20

2 Fluxos submodulares $\quad 23$

2.1 Matrizes de rede . . . . . . . . . . . . . . . . . . . 24

2.2 Famílias livres de cruzamentos e representações arbóreas . . . . . . . . . . 25

2.3 O teorema de Edmonds-Giles . . . . . . . . . . . . . . . . . . . . . . . . . . . . . . . .

2.4 Uma variante . . . . . . . . . . . . . . . . . . . . . . . . . . . . . . . . . . . . .

Resultados relacionados . . . . . . . . . . . . . . . 33

3 O algoritmo de Frank 35

3.1 Núcleos . . . . . . . . . . . . . . . . . . . . . . 36

3.2 Condições de otimalidade e potenciais . . . . . . . . . . . . . . 38

3.3 Um grafo auxiliar e circuitos axiais . . . . . . . . . . . . . . . . . . . 39

3.4 A iteração primal-dual . . . . . . . . . . . . . . . . . . . . . . . . . . . . . . . . . .

3.5 Menores núcleos justos . . . . . . . . . . . . . . . . . . . . . . . . . . . . . . . . . .

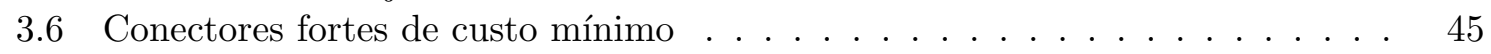

Resultados relacionados . . . . . . . . . . . . . . . 47 
4 Empacotamento de transversais de cortes $\quad 49$

4.1 O teorema das ramificações disjuntas de Edmonds . . . . . . . . . . . . . . 50

4.2 O teorema das árvores geradoras disjuntas de Tutte e Nash-Williams . . . . . 53

4.3 O teorema das bi-ramificações disjuntas de Schrijver . . . . . . . . . . . . . 56

$4.4 \mathrm{O}$ teorema das junções disjuntas . . . . . . . . . . . . . . 58

4.5 O teorema dos conectores fortes disjuntos de Schrijver . . . . . . . . . . 62

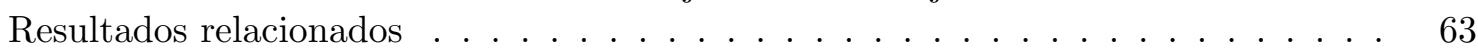

5 A conjectura de Edmonds-Giles $\quad \mathbf{6 5}$

5.1 Uma visão geral . . . . . . . . . . . . . . . . . . . . . . . . 66

5.2 Alguns casos especiais . . . . . . . . . . . . . . . . . . . . . . . . . . . . . . . . . . .

5.3 A conjectura dual planar . . . . . . . . . . . . . . . . . 72

5.4 Uma conjectura envolvendo subestruturas proibidas . . . . . . . . . . . 74

5.5 Empacotamento fracionário de junções . . . . . . . . . . . . . . 76

5.6 O teorema de Lee e Williams . . . . . . . . . . . . . . . . . . . 81

Resultados relacionados . . . . . . . . . . . . . . . . . . 89

$6 \quad T$-junções e emparelhamentos $\quad 91$

6.1 O teorema de Frank, Tardos e Sebö . . . . . . . . . . . . . . . . . . 92

6.2 Emparelhamentos . . . . . . . . . . . . . . . . . . 96

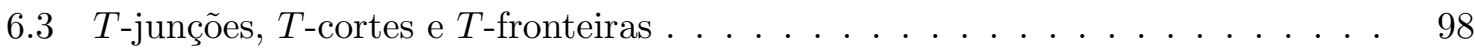

$6.4 \mathrm{Um}$ teorema de Seymour . . . . . . . . . . . . . . . . . . . . . . . 99

Resultados relacionados . . . . . . . . . . . . . . . . . . . 102

7 S-caminhos $\quad 107$

7.1 Um grafo rotulado por um grupo . . . . . . . . . . . . . . . . . 108

7.2 Bases de um matróide de $S$-caminhos _ . . . . . . . . . . . . . . . . 109

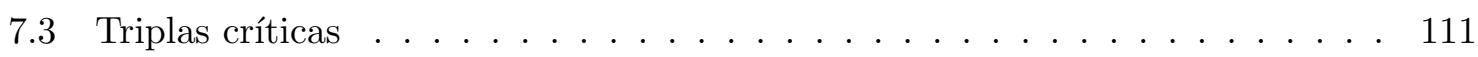

7.4 Uma relação min-max para $S$-caminhos não-nulos . . . . . . . . . . . . . 113

7.5 Conseqüências . . . . . . . . . . . . . . . . . . . . . 115

Resultados relacionados . . . . . . . . . . . . . . . . . 116

$\begin{array}{ll}\text { Considerações finais } & 117\end{array}$

$\begin{array}{ll}\text { Referências bibliográficas } & 119\end{array}$

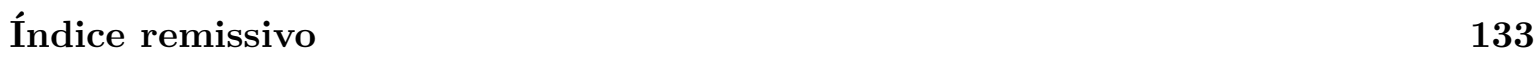




\section{Introdução}

Otimização combinatória é a área que tem como foco o estudo de problemas nos quais o objetivo é minimizar ou maximizar uma dada função sobre os elementos de um domínio discreto, tipicamente finito. Ainda que, geralmente, seja fácil enumerar todos os elementos do domínio para encontrar um elemento ótimo, na maioria das vezes essa abordagem ingênua está fadada ao fracasso, pois o tamanho do domínio cresce exponencialmente com relação ao tamanho da representação da entrada. Por isso são necessárias abordagens mais engenhosas.

Essa área está intimamente relacionada com programação linear, teoria da complexidade computacional e teoria dos grafos, e possui diversas sub-áreas, como por exemplo algoritmos de aproximação e combinatória poliédrica. O objetivo principal dessas sub-áreas é o desenvolvimento de algoritmos que resolvam de forma satisfatória alguns problemas reconhecidamente difíceis, ou seja, para os quais acredita-se ser improvável que existam algoritmos eficientes.

Neste texto, entretanto, não estamos interessados nesse tipo de problema. Nosso foco está nos problemas que admitem algoritmos eficientes, isto é, algoritmos cujo consumo de tempo seja limitado por um polinômio no tamanho da representação da entrada. Nos últimos 60 anos, o estudo de tais problemas levou ao desenvolvimento de uma rica e elegante teoria, que nos fornece uma melhor compreensão da estrutura combinatória dos problemas e, algumas vezes, nos auxilia na resolução de problemas difíceis, ainda que esse não seja o objetivo principal.

Suponha então que queremos desenvolver um algoritmo eficiente para um certo problema de otimização. Vamos usar o seguinte problema como exemplo: dado um grafo bipartido, encontre um emparelhamento de tamanho máximo.

Como podemos desenvolver nosso algoritmo? Poderíamos elaborar um procedimento que, dado um emparelhamento qualquer, tenta aplicar sobre ele alguma operação para torná-lo maior. Poderíamos então repetir esse procedimento até que ele não consiga mais encontrar um emparelhamento maior. A questão é: quando parar? Quando podemos ter certeza de que não existe nenhum emparelhamento maior? Será que a incapacidade do nosso procedimento de encontrar um emparelhamento maior implica que um tal emparelhamento não existe? Essa dúvida nos leva à busca por condições de otimalidade.

Se conseguíssemos obter um limitante superior para o tamanho de um emparelhamento e o nosso emparelhamento atingisse esse limitante, então teríamos certeza de que o nosso emparelhamento tem tamanho máximo. Felizmente, para uma vasta gama de problemas, é fácil obter limitantes. Em geral, formulamos um problema de minimização, que chamamos de dual, e que tem a seguinte propriedade: cada candidato a solução do problema dual fornece um limitante superior para o valor da solução do problema original. 
No nosso caso, um possível problema dual é o seguinte: dado um grafo bipartido, encontre uma cobertura por vértices de tamanho mínimo. É fácil ver que o tamanho de um emparelhamento nunca excede o tamanho de uma cobertura por vértices. Logo, o tamanho máximo de um emparelhamento nunca excede o tamanho mínimo de uma cobertura por vértices.

$\mathrm{Na}$ verdade, König provou que essa relação sempre vale com igualdade: em qualquer grafo bipartido, o tamanho mínimo de uma cobertura por vértices é igual ao tamanho máximo de um emparelhamento. Igualdades desse tipo são chamadas de relações min-max.

Relações min-max têm várias conseqüências importantes. Elas mostram que os problemas envolvidos estão tão interligados que muitas vezes é praticamente impossível resolver um dos problemas sem resolver simultaneamente o outro, ainda que de forma implícita.

Voltando ao nosso algoritmo, podemos tentar a seguinte abordagem, agora que obtemos uma condição de otimalidade da relação min-max de König. Se o nosso procedimento não é capaz de encontrar um emparelhamento maior, tente construir uma cobertura por vértices de mesmo tamanho do emparelhamento atual. Se isso for possível, então a cobertura encontrada certifica que o emparelhamento atual tem tamanho máximo. Na verdade, a prova de König fornece exatamente um algoritmo eficiente desse tipo.

A discussão anterior mostra ainda outra conseqüência importante da relação min-max de König: uma boa caracterização. Suponha que queiramos convencer alguém de que um certo emparelhamento não tem tamanho máximo. Para tanto, basta exibirmos um emparelhamento maior. Suponha agora que queremos convencer alguém de que um certo emparelhamento tem tamanho máximo. Não é razoável enumerarmos todos os emparelhamentos e mostrarmos que nenhum deles é maior que o emparelhamento em questão. Isso poderia levar muito tempo. Queremos um certificado sucinto da otimalidade do emparelhamento em questão. A relação min-max de König fornece exatamente uma solução para o nosso problema: basta exibirmos uma cobertura por vértices de mesmo tamanho que o emparelhamento.

A noção de boa caracterização, introduzida por Edmonds, pode ser descrita da seguinte forma. Suponha que tenhamos um candidato a solução para um problema de otimização. Se o candidato não é uma solução, existe um certificado sucinto disso: um candidato melhor. Se o candidato é uma solução, também existe um certificado sucinto desse fato.

A solução para o nosso problema foi bastante satisfatória. O algoritmo de König encontra eficientemente um emparelhamento de tamanho máximo num grafo bipartido e, como se isso não fosse bom o suficiente, ele ainda devolve uma cobertura por vértices que torna irrefutável a otimalidade do emparelhamento devolvido.

Soluções semelhantes existem para diversos outros problemas importantes em otimização combinatória, sendo a existência de relações min-max a característica unificadora. Em alguns casos, a prova da relação min-max já descreve implicitamente um algoritmo eficiente para resolver ambos os problemas em questão. Mesmo quando isso não ocorre, a relação min-max e sua prova revelam o suficiente sobre a estrutura combinatória dos problemas para que um algoritmo eficiente possa ser desenvolvido. Por isso, encontrar uma relação min-max para um problema é quase tão bom quanto construir um algoritmo eficiente para ele.

Pode-se dizer então que relações min-max são objetos centrais nesse ramo da otimização combinatória que estuda problemas eficientemente solúveis. Por essa razão, tais relações são o principal assunto deste texto. 


\section{Uma visão geral}

Estamos interessados em relações min-max para problemas de otimização sobre grafos, com ênfase sobre grafos orientados.

Alguns objetos centrais no nosso estudo são os cortes, ou seja, conjuntos de arcos que entram em determinados conjuntos de vértices. De acordo com esses subconjuntos de vértices, temos diferentes tipos de cortes. Por exemplo, se $r$ e $s$ são vértices de um grafo orientado, dizemos que um subconjunto de arcos é um $(r, s)$-corte se é exatamente o conjunto dos arcos que entram num certo subconjunto de vértices que possui $s$ mas não contém $r$.

Diversos problemas interessantes envolvem o estudo de tranversais de cortes, ou seja, conjuntos de arcos que intersectam todos os cortes de um determinado tipo. Objetos deste tipo podem ser vistos como conectores. Por exemplo, é fácil ver que as transversais minimais dos $(r, s)$-cortes correspondem aos $(r, s)$-caminhos, ou seja, os caminhos que ligam $r$ a $s$. Isso nos motiva a estudar o problema de encontrar transversais de custo mínimo. Existem várias relações min-max nessa linha, de acordo com o tipo de corte que escolhemos cobrir. Nelas, o problema de minimização envolve uma transversal de cortes de custo mínimo, e o de maximização, o empacotamento máximo dos cortes em questão, ou seja, queremos encontrar a maior coleção de cortes que são, num certo sentido, disjuntos. Essas relações são estudadas no capítulo 2, sobre fluxos submodulares.

Uma das relações min-max mais célebres sobre transversais de cortes de custo mínimo é o teorema de Lucchesi-Younger. Esse resultado é derivado no capítulo 2 de forma relativamente simples, ao custo de ser uma prova puramente existencial. Já o capítulo 3 é dedicado a um algoritmo fortemente polinomial para uma versão capacitada dessa relação min-max, desenvolvido por Frank. No fim desse capítulo, apresentamos um resultado de Schrijver que transforma esse algoritmo de Frank num algoritmo fortemente polinomial para quase todas as relações min-max derivadas no capítulo 2 .

No capítulo 4, estudamos relações min-max polares às anteriores, ou seja, que envolvem o empacotamento máximo de transversais de cortes. Nesse caso, o problema de minimização consiste em encontrar um corte de custo mínimo. Vamos ver que muitas das relações min-max vistas nos capítulos 2 e 3 continuam valendo após trocarmos os cortes e conectores nos problemas de otimização. Por exemplo, valem as seguintes relações min-max: 1) o número mínimo de arcos de um $(r, s)$-caminho é igual ao número máximo de $(r, s)$-cortes disjuntos; 2) o número mínimo de arcos de um $(r, s)$-corte é igual ao número máximo de $(r, s)$-caminhos disjuntos.

A relação min-max polar ao teorema de Lucchesi-Younger é um problema em aberto há quase 30 anos e é conhecida como conjectura de Woodall. Sua versão capacitada é conhecida como conjectura de Edmonds-Giles, que sabemos ser falsa. Porém, por ser válida para diversas classes de grafos, ela não é considerada fechada de forma satisfatória. Seria interessante, por exemplo, caracterizar exatamente para quais grafos ela é válida. No capítulo 5 , apresentamos uma resenha completa dos resultados já publicados sobre essas conjecturas.

Nos capítulos 6 e 7, passamos ao estudo de relações min-max mais clássicas, que envolvem grafos não-orientados. As principais relações min-max desse tipo referem-se ao problema do emparelhamento máximo e generalizações desse problema. Estudamos duas dessas generalizações: $T$-junções no capítulo 6 e $S$-caminhos no capítulo 7 . 


\section{Alguns aspectos recorrentes}

Alguns tipos e técnicas de prova têm sido usados repetidas vezes com sucesso para a obtenção de relações min-max. Nesta seção, vamos descrever brevemente alguns desses temas recorrentes para ter uma idéia melhor do que está por vir nos capítulos que se seguem.

Um tipo de prova bastante comum para provar relações min-max é o que faz uso de técnicas da combinatória poliédrica. Uma receita geral para provas desse tipo é a seguinte. Primeiro imergimos de alguma forma o conjunto de todos os candidatos a solução num espaço euclidiano de dimensão adequada, formando um certo conjunto $C$. Por exemplo, se os candidatos a solução forem os emparelhamentos de um grafo, o conjunto $C$ pode ser formado pelos vetores de incidência de emparelhamentos em $\mathbb{R}^{m}$, onde $m$ é o número de arestas do grafo. Feito isso, tomamos o fecho convexo dos pontos de $C$, obtendo um poliedro $P$. Agora o nosso problema de otimização pode ser formulado como um programa linear sobre $P$. Podemos agora usar técnicas da combinatória poliédrica para descobrir um sistema de inequações lineares $A x \leq b$ tal que $P=\{x: A x \leq b\}$. Podemos também estudar o programa linear dual para tentar provar que, nos casos relevantes, ele sempre admite uma solução ótima integral. Isso fornece imediatamente uma relação min-max. Um exemplo desse tipo de prova aparece no capítulo 2, sobre fluxos submodulares.

Outro tema recorrente em demonstrações de relações min-max é o uso de submodularidade. Se $f$ é uma função que associa um número a cada subconjunto de um conjunto $V$, dizemos que $f$ é submodular se $f(U \cap W)+f(U \cup W) \leq f(U)+f(W)$ para quaisquer $U, W \subseteq V$. Funções submodulares são "bem comportadas" de maneira semelhante às funções convexas. Uma função submodular particularmente importante para nós é a função $\vec{d}(S)$, que conta quantos arcos de um grafo orientado entram num certo subconjunto $S$ de vértices.

Vamos ver que, muitas vezes, uma condição necessária e suficiente para que um grafo orientado possua uma certa propriedade interessante é a de que $\vec{d}(S) \geq g(S)$ para todo $S \in \mathcal{S}$, onde $-g$ é uma função submodular e $\mathcal{S}$ é uma coleção de subconjuntos de vértices, como por exemplo a coleção dos subconjuntos não-vazios e próprios do conjunto de vértices do grafo orientado. Em geral, é fácil ver que a condição é necessária para a nossa propriedade. Por outro lado, provar sua suficiência costuma ser bem mais difícil. Digamos que temos um grafo orientado que satisfaz essa condição. Chamemos um conjunto $S \in \mathcal{S}$ de justo se $\vec{d}(S)=g(S)$. Os conjuntos justos podem ser vistos como "perigosos", no sentido de que devemos ser muito cuidadosos com eles. Por exemplo, se removermos um certo arco, a condição passa a ser violada exatamente quando esse arco entra em algum conjunto justo.

Para provar a suficiência da condição, muitas vezes é útil estudar as propriedades dos conjuntos justos. Nessa hora, a submodularidade das funções em questão torna nossa tarefa muito mais fácil. Por exemplo, se $U$ e $W$ são justos e $U \cap W, U \cup W \in \mathcal{S}$, então

$g(U)+g(W)=\vec{d}(U)+\vec{d}(W) \geq \vec{d}(U \cap W)+\vec{d}(U \cup W) \geq g(U \cap W)+g(U \cup W) \geq g(U)+g(W)$,

de modo que todas as inequações acima valem com igualdade, e portanto $U \cap W$ e $U \cup W$ também são justos. Suponha que, com essa técnica, provamos que, sempre que $U$ e $W$ são justos e se intersectam, então $U \cap W$ também é justo. Isso implica que, se $U$ é um conjunto justo minimal e $W$ é outro conjunto justo que intersecta $W$, então $U \subseteq W$. Propriedades 
desse tipo, bem como outras propriedades que fazem uso da união no lugar da intersecção, são muitas vezes fortes o suficiente para que possamos construir sobre elas um argumento que prova a suficiência da condição. Veja, por exemplo, as provas do capítulo 4, como por exemplo a prova do teorema das ramificações disjuntas de Edmonds e a do teorema das árvores geradoras disjuntas de Tutte e Nash-Williams.

Uma técnica relacionada ao uso de funções submodulares é a técnica do descruzamento. Algumas vezes, estamos interessados em obter uma certa coleção $\mathcal{C}$ de subconjuntos de vértices sendo que exigimos alguma condição envolvendo $\vec{d}(S)$ para todo $S \in \mathcal{C}$. Usando o fato de que a função $\vec{d}(S)$ é submodular, podemos encontrar uma tal coleção $\mathcal{C}$ que seja particularmente "bem estruturada", no seguinte sentido: dados dois membros de $\mathcal{C}$ que se intersectam, então ou um deles está contido no outro ou então sua união é o conjunto de todos os vértices. Nesse caso, dizemos que $\mathcal{C}$ é livre de cruzamentos. Para ver como isso impõe uma estrutura forte sobre $\mathcal{C}$, suponha que nenhum dos membros de $\mathcal{C}$ possui um certo vértice do grafo. Então a coleção $\mathcal{C}$ é laminar, ou seja, sempre que dois membros de $\mathcal{C}$ se intersectam, um deles está contido no outro. Essa estrutura forte tem se mostrado muito útil para a construção de argumentos e aparece em provas de relações min-max bastante gerais. Por exemplo, a técnica do descruzamento é central para o principal teorema do capítulo 2, sobre fluxos submodulares.

Vimos que funções submodulares podem impor uma estrutura que facilita a solução de alguns problemas. Outros objetos que têm esse efeito são os matróides. Alguns sistemas de conjuntos relacionados a matróides satisfazem um tipo de propriedade muito especial: as propriedades de troca. Por exemplo, digamos que $\mathcal{B}$ é uma coleção de subconjuntos de um certo conjunto-base e que todos os membros de $\mathcal{B}$ têm o mesmo tamanho. Sejam $B$ e $B^{\prime}$ membros de $\mathcal{B}$. Uma possível propriedade de troca é a seguinte: para qualquer subconjunto $S$ de $B$, existe um subconjunto $S^{\prime}$ de $B^{\prime}$ tal que $(B \backslash S) \cup S^{\prime}$ e $\left(B^{\prime} \backslash S^{\prime}\right) \cup S$ são membros de $\mathcal{B}$. Essa propriedade, por exemplo, é satisfeita pela coleção das bases de um matróide qualquer. Aplicada ao matróide gráfico de um grafo conexo, obtemos o seguinte: para quaisquer árvores geradoras $T$ e $T^{\prime}$ de um grafo e qualquer subconjunto de arestas $F$ de $T$, existe um subconjunto de $\operatorname{arestas} F^{\prime}$ de $T^{\prime}$ tal que, se trocarmos $F$ por $F^{\prime}$ em $T$ e $F^{\prime}$ por $F$ em $T^{\prime}$, obtemos duas árvores geradoras desse grafo. Propriedades desse tipo são muito úteis quando temos em mãos dois objetos $B$ e $B^{\prime}$ que não são bons o suficiente, mas que se tornariam bons se redistribuíssemos alguns elementos entre eles. Veja, por exemplo, a prova do teorema das bi-ramificações disjuntas de Schrijver, no capítulo 4. Veja também o capítulo 7, que faz uso de matróides.

Resultados envolvendo subestruturas proibidas também são muito comuns. Algumas vezes ocorre o seguinte fenômeno. Uma certa afirmação não é válida, e é fácil encontrar um grafo que a viola. Além disso, sempre que esse grafo específico aparece como uma subestrutura de um outro grafo, este também viola a afirmação. A ocorrência do grafo específico como subestrutura pode ser na forma de um subgrafo induzido, de um subgrafo ou de um menor. Esse grafo específico é uma obstrução para a validade da afirmação. Note que pode haver várias outras obstruções. Depois de coletar várias obstruções numa certa coleção de grafos $\mathcal{G}$, podemos conjecturar o seguinte: se proibirmos que os grafos de $\mathcal{G}$ sejam subestruturas de um certo grafo, então tal grafo satisfaz a afirmação. Em outras palavras, se um grafo não possui nenhum dos grafos de $\mathcal{G}$ como subestrutura, então ele satisfaz a afirmação. Claramente, 
resultados dessa natureza são interessantes apenas quando a coleção $\mathcal{G}$ é pequena. Existem muitos teoremas desse tipo, dentre os quais encontramos várias relações min-max.

Em geral, as provas de resultados desse tipo têm o seguinte roteiro. Primeiro selecionamos um grafo minimal que viola a afirmação do teorema, sendo que a minimalidade depende do tipo de subestrutura que estamos proibindo. Usando o fato de que o grafo viola a afirmação, descobrimos o suficiente sobre sua estrutura para encontrar alguma das obstruções como subestrutura. Provas desse tipo costumam ser bastante técnicas. Elas também costumam ter um sabor de teoria dos grafos. Incluem-se nessa categoria o teorema de Lee e Williams, sobre um caso especial da conjectura de Edmonds-Giles (capítulo 5), e um teorema de Seymour sobre $T$-junções (capítulo 6). 


\section{Abordagem e escolha dos tópicos}

Existe uma vasta gama de relações min-max. É claro que apenas uma pequena porção dessas relações e da teoria relacionada pode ser abordada numa dissertação de mestrado sem que seu tamanho ultrapasse os limites do bom senso. Felizmente, existem alguns resultados bastante gerais a partir dos quais podemos derivar várias relações min-max com muita facilidade. Alguns desses resultados gerais são arcabouços, isto é, relações min-max nas quais podemos "encaixar algumas peças" para derivar outras relações min-max. Outros resultados não têm esse formato, mas são poderosos o suficiente para derivarmos deles outras relações sem muito trabalho. Por questões de espaço e para cobrir uma grande quantidade de tópicos, decidimos dar preferência a esses resultados gerais, nos casos em que suas provas não são demasiadamente longas ou complicadas. Surpreendentemente, existem teoremas desse tipo com provas bastante simples e acessíveis.

Essa preferência tem o seguinte defeito: as relações entre os vários resultados são omitidas. Por exemplo, vamos provar no capítulo 6 um poderoso teorema de Frank, Tardos e Sebö, a partir do qual todos os demais resultados do capítulo (e são muitos) podem ser derivados. Dentre esses resultados, está o teorema do casamento de Hall, um teorema bastante simples provado em 1935. Acontece, porém, que por resultados de Anderson [1971] e Szigeti [2004], o teorema de Frank, Tardos e Sebő pode ser obtido a partir do teorema do casamento de Hall e portanto esses resultados são equivalentes! Muitas outras relações desse tipo ficam omitidas, inclusive entre os capítulos, de modo que cada capítulo se torna independente dos outros. Observamos ao leitor interessado que existem muitas outras relações interessantes entre os diversos resultados deste texto que não tivemos a oportunidade de mencionar. Algumas dessas relações estão ilustradas na figura da página 118. Para guiar o leitor um pouco melhor na sua incursão à literatura, incluímos ao fim de cada capítulo uma seção chamada "Resultados relacionados" listando diversas referências.

Também procuramos dar um enfoque maior a tópicos relacionados a grafos orientados. A razão para isso é o fato de que as relações min-max clássicas em grafos não-orientados, como por exemplo as que envolvem emparelhamentos e caminhos disjuntos, são amplamente cobertas na literatura, até mesmo por livros básicos sobre algoritmos. Já os resultados sobre grafos orientados são muito menos conhecidos.

Um tópico em específico recebeu atenção especial: a conjectura de Woodall e sua versão capacitada, conhecida como conjectura de Edmonds-Giles. A conjectura de Woodall continua em aberto há quase 30 anos e a de Edmonds-Giles, apesar de já sabermos ser falsa, não é considerada fechada de forma satisfatória. Por um longo período, nada foi publicado sobre essas conjecturas, até que, mais recentemente, alguns pesquisadores voltaram sua atenção para elas. Entretanto, não encontramos na literatura nenhuma resenha completa sobre essas

conjecturas. No capítulo 5, tentamos elaborar uma tal resenha. Consideramos que esse capítulo é uma das contribuições principais deste texto, pois cobrimos todos os resultados já publicados sobre as conjecturas, sendo quase todos eles acompanhados de provas. 


\section{Observações}

Cabem algumas observações gerais acerca do texto.

Como já mencionamos, uma possível abordagem para um problema de otimização consiste em formulá-lo como um programa linear. Nesse caso, ao obtermos um sistema de inequações que determina o poliedro em questão, o teorema de dualidade em programação linear nos fornece automaticamente uma relação min-max. Por exemplo, se o poliedro é determinado pelo sistema $A x \leq b$, então

$$
\max \{c x: A x \leq b\}=\min \{y b: y \geq 0, y A=c\} .
$$

Não estamos interessados nesse tipo de relação min-max, pois o vetor y que atinge o mínimo pode ser formado por componentes fracionários. Em termos combinatórios, uma tal relação pode não ter uma "interpretação" tão simples. Compare, por exemplo, com a relação min-max de Kőnig: num grafo bipartido, o tamanho mínimo de uma cobertura por vértices é igual ao tamanho máximo de um emparelhamento. Nessa relação, ambos os problemas de otimização têm uma interpretação combinatória clara. Já se conseguirmos garantir que sempre existe um vetor integral $y$ que atinge o mínimo, podemos obter uma interpretação combinatória do problema de minimização. Nesse caso, a equação de dualidade nos interessa, e muito, pois mostra que a relação min-max vale também numa versão capacitada.

Mencionamos também que relações min-max em geral fornecem boas caracterizações dos problemas em questão. Observamos, porém, que isso nem sempre é verdade. Por exemplo, Seymour e Thomas [1993] obtiveram uma relação min-max para largura arbórea que não fornece uma boa caracterização, no sentido de que não prova que os problemas estão na classe de complexidade $\mathbf{N P} \cap \mathbf{c o N P}$, a não ser que $\mathbf{N P}=\mathbf{c o N P}$. Lembramos que o problema de determinar a largura arbórea de um grafo é NP-difícil. Por outro lado, não encontramos na literatura nenhuma outra relação min-max que não fornece uma boa caracterização.

Também podemos ter problemas com boas caracterizações no caso de versões capacitadas de relações min-max. Geralmente, tais relações envolvem coleções de subconjuntos de vértices e não parece haver nenhuma razão que impeça que essas coleções sejam formadas por todos os subconjuntos possíveis, ou sequer por uma quantidade excessivamente grande (exponencial) de subconjuntos. Para as relações min-max capacitadas que aparecem neste texto, isso não será um problema: todas as relações desse tipo aqui tratadas podem ser formuladas como programas lineares e, nesse caso, a validade da relação min-max é exatamente o mesmo que afirmar que um certo sistema de inequações lineares é totalmente dual integral, de modo que somos salvos por um teorema de CoOK, Fonlupt E SchriJver [1986], que pode ser visto na seção 1.5.

Para quase todas as relações min-max, a prova de que o máximo nunca excede o mínimo é absolutamente trivial. Omitiremos a prova desse fato a não ser que ela não seja imediata.

Cada resultado apresentado neste texto possui uma numeração única, independentemente de ser enunciado como lema, proposição, teorema ou corolário. Portanto, esse número pode ser usado para localizar o resultado. Por exemplo, o teorema de Lucchesi-Younger é apresentado como o corolário 2.3.1. Apesar disso, muitas vezes nos referimos a esse resultado como "teorema 2.3.1 de Lucchesi-Younger". Isso não deverá causar problemas. 


\section{Capítulo 1}

\section{Preliminares}

A terminologia e a notação que usamos neste texto é bastante padrão. Porém, achamos conveniente apresentá-las para não gerar dúvidas, e também para estabelecer certas convenções. Este é o papel deste capítulo.

$\mathrm{Na}$ seção 1.1, definimos as preliminares gerais; na seção 1.2, estabelecemos a notação para teoria dos grafos; na seção 1.3, definimos diversos conceitos sobre grafos orientados; na seção 1.4, definimos alguns conceitos sobre hipergrafos; na seção 1.5, relembramos um pouco de programação linear e inteira e derivamos alguns resultados que serão usados no capítulo 2; finalmente, na seção 1.6, desenvolvemos tudo o que precisaremos da teoria de matróides.

Recomendamos a leitura apenas das duas última seções. As demais podem ser consultadas de acordo com as necessidades do leitor.

Tentamos manter o texto o mais auto-contido possível. Os poucos resultados que não provamos aqui são bastante acessíveis. Procuramos incluir as provas de alguns resultados que não consideramos tão conhecidos, como por exemplo alguns resultados básicos de teoria dos matróides. Notamos que apenas o capítulo 7 usa conceitos de teoria dos matróides.

Pressupomos que o leitor está familiarizado com alguns conceitos básicos de análise de algoritmos e de complexidade computacional, como por exemplo as definições de algoritmo polinomial e fortemente polinomial, alguns algoritmos básicos sobre grafos e algumas classes de complexidade, como a dos problemas NP-difíceis. O leitor interessado pode consultar os livros de Cormen, Leiserson, Rivest e Stein [2001] e de Schrijver [2003].

Para mais detalhes sobre grafos e grafos orientados, indicamos os livros de Diestel [2005] e de SchriJver [2003]. Um tratamento mais preciso de grafos planares pode ser visto nos livros de Diestel [2005] e de Mohar e Thomassen [2001]. Uma ótima referência para programação linear e inteira é o livro de SchriJver [1986]. Para leitores interessados em teoria dos matróides, indicamos os livros de WELSH [1976] e OXLEY [1992]. 


\subsection{Preliminares gerais}

O conjunto dos números inteiros é denotado por $\mathbb{Z}$, o dos racionais por $\mathbb{Q}$ e o dos reais por $\mathbb{R}$. Podemos adicionar o índice $\geq 0$ a um conjunto para nos referir aos elementos não-negativos desse conjunto. Por exemplo, $\mathbb{Z}_{\geq 0}$ denota o conjunto dos inteiros não-negativos.

Em geral, os conjuntos tratados neste texto são finitos. Isso deve ficar subentendido. Por exemplo, se dissermos que $V$ é um conjunto, fica implícito que $V$ é finito. As únicas exceções para essa regra são as óbvias, como por exemplo o conjunto $\mathbb{Z}$ e variantes.

Algumas vezes confundimos um conjunto unitário com seu único elemento.

Um par é um par ordenado.

Ao longo desta seção, $V$ denota um conjunto.

O tamanho de $V$ é o número de elementos de $V$ e é denotado por $|V|$. Escrevemos $S \subsetneq V$ ou $V \supsetneq S$ para indicar que $S \subseteq V$ e $S \neq V$. A coleção dos subconjuntos de $V$ é denotada por $\mathcal{P}(V)$. Um elemento $z$ é novo (com relação a $V$ ) se $z \notin V$. Dizemos que alguns conjuntos são disjuntos se eles são mutuamente disjuntos. A diferença simétrica de $U$ e $W$ é $U \triangle W:=(U \backslash W) \cup(W \backslash U)$.

Seja $\mathcal{S}$ uma coleção de subconjuntos de $V$. Denotamos $\bigcup \mathcal{S}:=\bigcup_{S \in \mathcal{S}} S$. Dizemos que a coleção $\mathcal{S}$ é disjunta se $U$ e $W$ são disjuntos para quaisquer $U, W \in \mathcal{S}$ distintos. Se $\mathcal{S}$ é uma coleção disjunta, $S \neq \varnothing$ para todo $S \in \mathcal{S}$ e $\bigcup \mathcal{S}=V$, dizemos que $\mathcal{S}$ é uma partição de $V$ e chamamos cada $S \in \mathcal{S}$ de uma parte de $\mathcal{S}$.

Seja $f: \mathcal{P}(V) \rightarrow \mathbb{R}$ uma função. Dizemos que $f$ é submodular se

$$
f(U \cap W)+f(U \cup W) \leq f(U)+f(W)
$$

para quaisquer $U, W \subseteq V$. Dizemos que $f$ é supermodular se $-f$ é submodular.

Ao longo do texto vamos definir funções $f: V \rightarrow \mathbb{Z}$ e vamos dizer que $f$ é uma função capacidade, peso, custo, etc. É óbvio que essa nomenclatura não tem qualquer significado matemático e serve simplesmente para, digamos, nos referirmos a $f(v)$ como o peso de um elemento $v \in V$, a $f(S)$ como o custo de um subconjunto $S \subseteq V$, etc.

Um vetor indexado por $V$ (ou simplesmente vetor, se o conjunto $V$ estiver claro no contexto) é uma função $x: V \rightarrow \mathbb{R}$. Dizemos que $x$ é integral se $x(v) \in \mathbb{Z}$ para todo $v \in V$. $\mathrm{O}$ suporte de $x$ é $\left\{v \in V: x_{v} \neq 0\right\}$. Seja $v \in V$. Algumas vezes escrevemos $x_{v}$ no lugar de $x(v)$. Dizemos que $x_{v}$ é um componente de $x$. Denotamos por $\mathbb{R}^{V}$ o conjunto dos vetores indexados por $V$ e por $\mathbb{Z}^{V}$ o conjunto dos vetores integrais de $\mathbb{R}^{V}$.

Seja $S \subseteq V$. O vetor de incidência de $S$ é o vetor $\chi^{S} \in \mathbb{R}^{V}$ definido da seguinte maneira:

$$
\chi^{S}(v):= \begin{cases}1, & \text { se } v \in S, \\ 0, & \text { caso contrário. }\end{cases}
$$

Sejam $x, y \in \mathbb{R}^{V}$. Denotamos por $x y$ o número $\sum_{v \in V} x_{v} y_{v}$. Abreviamos também

$$
x(S):=x \chi^{S} .
$$

Sejam $U$ e $W$ conjuntos. Uma matriz $M$ sobre $U \times W$ é um vetor indexado por $U \times W$. Algumas vezes escrevemos $M[u, w]$ no lugar de $M_{(u, w)}$. Se $M$ é quadrada, isto é, se 
$|U|=|W|$, denotamos o determinante de $M \operatorname{por} \operatorname{det}(M)$. Note que, para que $\operatorname{det}(M)$ esteja bem definido, é preciso especificar uma bijeção entre $U$ e $W$. Se não especificarmos uma tal bijeção, apenas o valor absoluto $\operatorname{de} \operatorname{det}(M)$ fica bem definido. Porém, todas as propriedades que nos interessam sobre $\operatorname{det}(M)$ independem de seu sinal, e portanto não precisamos nos preocupar com a bijeção entre $U$ e $W$ usada para definir $\operatorname{det}(M)$. Dizemos que uma matriz $M$ é totalmente unimodular se $\operatorname{det}\left(M^{\prime}\right) \in\{0, \pm 1\}$ para toda submatriz quadrada $M^{\prime}$ de $M$.

Seja $x \in \mathbb{R}^{W}$. Denotamos por $M x$ o vetor $x^{\prime}$ de $\mathbb{R}^{U}$ definido da seguinte forma. Para cada $u \in U$, defina $x_{u}^{\prime}:=\sum_{w \in W} M[u, w] x_{w}$. Seja $y \in \mathbb{R}^{U}$. Denotamos por $y M$ o vetor $y^{\prime}$ de $\mathbb{R}^{W}$ definido da seguinte forma. Para cada $w \in W$, defina $y_{w}^{\prime}:=\sum_{u \in U} y_{u} M[u, w]$.

Uma família é um multiconjunto, ou seja, um conjunto que pode conter diversas cópias indistinguíveis de um mesmo elemento. O número de cópias de um certo elemento que a família possui é a multiplicidade desse elemento.

Todas as operações sobre famílias levam em conta o fato de que elas podem possuir várias cópias indistinguíveis de um mesmo elemento. Por exemplo, digamos que todos os elementos de uma família $\mathcal{F}$ são membros de um conjunto $V$. Defina um vetor $\chi^{\mathcal{F}} \in \mathbb{R}^{V}$ da seguinte forma: para cada $v \in V$, tome como $\chi^{\mathcal{F}}(v)$ a multiplicidade de $v$ em $\mathcal{F}$. Note que isso fornece uma correspondência óbvia entre famílias com elementos em $V$ e vetores em $\mathbb{Z}_{\geq 0}^{V}$.

Com essa correspondência em mente, definimos o tamanho da família como $\chi^{\mathcal{F}}(V)$. Se $\mathcal{G}$ é outra família com elementos em $V$, então $\mathcal{F} \cup \mathcal{G}$ é a família correspondente ao vetor $\chi^{\mathcal{F}}+\chi^{\mathcal{G}}$.

Seja $\mathcal{S}$ uma família de subconjuntos de $V$ e $f: V \rightarrow \mathbb{Z}_{\geq 0}$ uma função. Dizemos que $\mathcal{S}$ é $f$-disjunta se cada elemento $v \in V$ aparece em no máximo $f(v)$ membros de $\mathcal{S}$.

\subsection{Grafos}

Um grafo é um par $G=(V, E)$, onde $V$ e $E$ são conjuntos disjuntos, e uma função implícita $\psi_{G}$ atribui a cada elemento de $E$ um subconjunto de $V$ de tamanho 1 ou 2 . Os elementos de $V$ são chamados de vértices, e os de $E$, de arestas. Dizemos que $V$ é o conjunto de vértices de $G$ e que $E$ é o conjunto de arestas de $G$. Dizemos que $G$ é um grafo sobre $V$. Algumas vezes denotamos o conjunto de vértices de $G$ por $V_{G}$ ou por $V(G)$ e o conjunto de arestas de $G$ por $E_{G}$ ou por $E(G)$.

Diversos outros parâmetros de grafos serão denotados por símbolos com o índice $G$ se $G$ for o grafo em questão. Sempre que $G$ estiver implícito no contexto, podemos omitir o índice $G$. Por exemplo, se estamos tratando de um único grafo, o índice ${ }_{G}$ pode ser omitido.

Se $e \in E$ e $\psi(e)=\{u, v\}$, onde possivelmente $u=v$, dizemos que a aresta $e$ liga $u$ a $v$ e que $u$ e $v$ são as pontas de $e$. Dizemos também que uma aresta incide ou é incidente em suas pontas. Dizemos ainda que $u$ é vizinho de $v$. Se $S \subseteq V$, denotamos por $N_{G}(S)$ o conjuntos dos vértices de $V \backslash S$ que têm algum vizinho em $S$.

Nos demais capítulos não vamos mais mencionar a função implícita $\psi$ e, a partir de agora, vamos abreviar o fato de que $\psi(e)=\{u, v\}$ escrevendo $e=u v$. Assim, quando nos referirmos a "uma aresta $u v$ ", seríamos mais precisos se disséssemos "uma aresta do tipo $u v$ ". A função implícita $\psi$ permite que existam diversas arestas com as mesmas pontas, e será conveniente enxergarmos cada aresta como um conjunto formado por 1 ou 2 vértices que é "rotulado" pelo "nome da aresta". 
Observe que podem existir arestas que têm um único vértice como ponta. Tais arestas são chamadas de laços. Se duas arestas que não são laços têm as mesmas pontas, dizemos que elas são paralelas. Um grafo que não possui nem laços e nem arestas paralelas é simples.

No restante da seção, $G=(V, E)$ denota um grafo.

Seja $v \in V$. Denote por $E^{\prime}$ o conjunto de arestas de $E$ que não são laços. O grau de $v$ em $G$ é $g_{G}(v):=\mid\left\{e \in E^{\prime}: e\right.$ incide em $\left.v\right\}|+2|\left\{e \in E \backslash E^{\prime}: e\right.$ incide em $\left.v\right\} \mid$. Se $F \subseteq E$, então $g_{F}(v)$ denota o grau de $v$ no grafo $(V, F)$. Um vértice é isolado se seu grau é nulo. O grau mínimo de $G$ é $\delta(G):=\min _{v \in V} g(v)$ e o grau máximo de $G$ é $\Delta(G):=\max _{v \in V} g(v)$. Se $\delta(G)=\Delta(G)=k$, dizemos que $G$ é $k$-regular ou simplesmente regular. Se $G$ é 3-regular, dizemos que $G$ é cúbico. Se $G$ é simples, a densidade de $G$ é a razão $|E| /\left(\begin{array}{c}|V| \\ 2\end{array}\right)$.

Seja $S \subseteq V$ e $F \subseteq E$. Denotamos por $\delta_{F}(S)$ o conjunto das arestas de $F$ que têm uma ponta em $S$ e outra fora de $S$. Abreviamos $d_{F}(S):=\left|\delta_{F}(S)\right|$. Abreviamos $\delta_{G}(S):=\delta_{E}(S)$ e $d_{G}(S):=d_{E}(S)$. Um simples argumento de contagem mostra que, para $F \subseteq E$ fixo, a função $d_{F}(S)$ é submodular.

Seja $H$ um grafo. Dizemos que $H$ é um subgrafo de $G$ se $V_{H} \subseteq V_{G}, E_{H} \subseteq E_{G}$ e $\psi_{H}$ é a restrição de $\psi_{G}$ a $E_{H}$. Nesse caso dizemos também que $G$ é um supergrafo de $H$. Dizemos que o subgrafo $H$ é gerador se $V_{H}=V_{G}$. Seja $S \subseteq V$. Denote por $E_{G}[S]$ o conjunto de todas as arestas de $G$ com as duas pontas em $S$. O subgrafo de $G$ induzido por $S$ é o subgrafo $G[S]:=(S, E[S])$. Um subgrafo $H$ de $G$ é um subgrafo induzido de $G$ se $H=G\left[V_{H}\right]$. Denotamos $G-S:=G[V \backslash S]$. Se $F \subseteq E$, denotamos $G-F:=(V, E \backslash F)$.

Um caminho em $G$ é uma seqüência $P=\left\langle v_{0}, e_{1}, v_{1}, \ldots, e_{k}, v_{k}\right\rangle$ tal que $v_{0}, \ldots, v_{k} \in V$ são distintos e $e_{i} \in E$ liga $v_{i-1}$ a $v_{i}$ para $i=1, \ldots, k$. Dizemos que $v_{0}$ e $v_{k}$ são as extremidades de $P$, que $P$ vai de $v_{0}$ a $v_{k}$, que $P$ liga $v_{0}$ a $v_{k}$ em $G$, que $v_{k}$ é acessível a partir de $v_{0}$ em $G$ e que $v_{0}$ acessa $v_{k}$ em $G$. O comprimento de $P$ é $k$. Algumas vezes abreviamos $P=v_{0} \cdots v_{k}$. Abreviamos também $V_{P}:=\left\{v_{0}, \ldots, v_{k}\right\}$ e $E_{P}:=\left\{e_{1}, \ldots, e_{k}\right\}$. Algumas vezes confundimos propositalmente o caminho $P$ com o grafo $\left(V_{P}, E_{P}\right)$ ou com o conjunto $E_{P}$.

Um circuito em $G$ é uma seqüência $C=\left\langle v_{0}, e_{1}, v_{1}, \ldots, e_{k}, v_{k}\right\rangle$ tal que $k \geq 1, v_{0}=v_{k}$, $\left\langle v_{0}, e_{1}, v_{1}, \ldots, e_{k-1}, v_{k-1}\right\rangle$ é um caminho em $G$ e as pontas da aresta $e_{k} \in E$ são $v_{k-1}$ e $v_{k}$. O comprimento de $C$ é $k$. Dizemos que $C$ é par se $k$ é par e é ímpar caso contrário. Algumas vezes abreviamos $C=v_{0} \cdots v_{k}$. Abreviamos também $V_{C}:=\left\{v_{0}, \ldots, v_{k-1}\right\}$ e $E_{C}:=$ $\left\{e_{1}, \ldots, e_{k}\right\}$. Algumas vezes confundimos propositalmente o circuito $C$ com o grafo $\left(V_{C}, E_{C}\right)$ ou com o conjunto $E_{C}$. Dizemos que $G$ é acíclico se não possui circuitos. Nesse caso, dizemos também que $G$ é uma floresta. Algumas vezes confundimos propositalmente uma floresta com seu conjunto de arestas.

Um componente de $G$ é um subgrafo $H$ de $G$ maximal tal que, para quaisquer vértices $x, y \in V_{H}, x$ acessa $y$ em $H$. Algumas vezes chamamos de componente o conjunto de vértices de um componente.

Dizemos que $G$ é conexo se possui um único componente e que $G$ é uma árvore se é conexo e acíclico. É fácil provar que $\left|E_{T}\right|=\left|V_{T}\right|-1$ para toda árvore $T$. Um subgrafo gerador de $G$ que é uma árvore é uma árvore geradora de $G$.

Seja $k \in \mathbb{Z}_{\geq 0}$. Dizemos que $G$ é $k$-conexo se $|V|>k$ e $G-S$ é conexo para qualquer $S \subseteq V$ tal que $|S|<k$. Dizemos que $G$ é $k$-aresta-conexo se $G-F$ é conexo para qualquer $F \subseteq E$ tal que $|F|<k$. 
Uma bipartição de $G$ é uma coleção $\{U, W\}$ de subconjuntos de $V$ tal que $U$ e $W$ são disjuntos, $U \cup W=V$ e toda aresta de $G$ tem uma ponta em $U$ e outra em $W$. Se $\{U, W\}$ é uma bipartição de $G$, dizemos que $G$ é $(U, W)$-bipartido. Dizemos que $G$ é bipartido se possui uma bipartição. Se $\{U, W\}$ é uma bipartição de $G$, chamamos $U$ e $W$ de classes de cor de $G$, apesar da escolha da bipartição $\{U, W\}$ não ser única. É óbvio que um grafo é bipartido se, e somente se, não possui um circuito ímpar.

Seja $S \subseteq V$ um subconjunto não-vazio. A contração de $S$ consiste na operação que transforma $G$ no grafo $G^{\prime}=\left(V^{\prime}, E\right)$ definido da seguinte maneira. Tome $V^{\prime}:=(V \backslash S) \cup\{S\}$. Altere a função $\psi_{G}$ da seguinte maneira para obter $\psi_{G^{\prime}}$. Seja $e=u v \in E$. Se $u, v \in S$, tome $\psi_{G^{\prime}}(e):=\{S\}$. Se $u \in S$ e $v \notin S$, tome $\psi_{G^{\prime}}(e):=\{S, v\}$. Para as demais arestas $e \in E$, tome $\psi_{G^{\prime}}(e):=\psi_{G}(e)$. Denotamos por $G / S$ o grafo $G^{\prime}$.

Seja $e=u v \in E$ uma aresta que não é um laço. A contração da aresta $e$ consiste na operação que transforma $G$ no grafo $(G-e) /\{u, v\}$. Se $F \subseteq E$, denotamos por $G / F$ o grafo obtido a partir de $G$ através da contração das arestas de $F$. Note que o grafo resultante é o mesmo, independente da ordem de contração de arestas.

Seja $H$ um grafo. Dizemos que $H$ é um menor de $G$ se algum grafo isomorfo a $H$ pode ser obtido a partir de algum subgrafo de $G$ através de contrações de arestas, onde o isomorfismo entre grafos é definido da maneira usual. Dizemos que $G$ é livre de $H$ como menor se nenhum menor de $G$ é isomorfo a $H$.

Dizemos que $G$ é completo se, para cada par de vértices $u, v \in V$ distintos, existe exatamente uma aresta de $G$ com pontas $u$ e $v$. Existe um único grafo completo que possui $V$ como conjunto de vértices. Tal grafo é denotado por $K_{V}$. Existe um único grafo completo com $n$ vértices, a menos de isomorfismo. Tal grafo é denotado por $K_{n}$. Seja $\{U, W\}$ uma partição de $V$. Denotamos por $K_{U, W}$ o grafo $(U, W)$-bipartido que possui precisamente uma aresta $u w$ para cada par de vértices $u \in U$ e $w \in W$. Existe um único grafo bipartido cujas classes de cores têm tamanhos $m$ e $n$, a menos de isomorfismo. Tal grafo é denotado por $K_{m, n}$.

Seja $H=(W, F)$ um grafo. Definimos $G \cup H:=(V \cup W, E \cup F)$. Denotamos por $G \times H$ o grafo $G \cup H \cup K_{V, W}$, onde supomos que $V$ e $W$ são disjuntos.

Dizemos que $M \subseteq E$ é um emparelhamento se $M$ não possui laços e cada vértice de $G$ é ponta de no máximo uma aresta de $M$.

A matriz de incidência de $G$ é a matriz $M$ sobre $V \times E$ definida da seguinte forma. Para cada vértice $v \in V$ e cada aresta $e \in E$, defina

$$
M[v, e]:= \begin{cases}1, & \text { se } v \text { é ponta de } e, \\ 0, & \text { caso contrário. }\end{cases}
$$

Dizemos que $G$ é planar se admite uma imersão no plano. Fixe uma imersão $|G|$ de $G$ no plano. As faces de $|G|$ são os componentes topológicos de $\mathbb{R}^{2} \backslash|G|$. O grafo dual de $G$ (com relação à imersão $|G|$ ) é o grafo $G^{*}$ sobre o conjunto das faces de $|G|$ e conjunto de arestas $E^{*}:=\left\{e^{*}: e \in E\right\}$, onde, para cada $e \in E$, as pontas de $e^{*}$ em $G^{*}$ são as faces de $|G|$ que têm $e$ em sua fronteira.

Pelos resultados de Kuratowski [1930] e Wagner [1937a], sabemos que um grafo é planar se, e somente se, é livre de $K_{5}$ e de $K_{3,3}$ como menores. 
Note que o conceito de dual de um grafo é relativo a uma dada imersão. Whitney [1932] mostrou que todo grafo planar 3-conexo possui essencialmente uma única imersão no plano. Assim, tais grafos possuem um único grafo dual. Uma prova curta desse resultado pode ser vista no livro de Diestel [2005].

\subsection{Grafos orientados}

Um grafo orientado é um par $D=(V, A)$, onde $V$ e $A$ são conjuntos disjuntos e uma função implícita $\psi_{D}$ atribui a cada elemento de $A$ um par de elementos de $V$. Os elementos de $V$ são chamados de vértices e os de $A$, de arcos. Dizemos que $V$ é o conjunto de vértices de $D$ e que $A$ é o conjunto de $\operatorname{arcos}$ de $D$. Algumas vezes denotamos o conjunto de vértices de $D$ por $V_{D}$ ou por $V(D)$ e o conjunto de arcos de $D$ por $A_{D}$ ou por $A(D)$.

O mesmo que dissemos para a omissão do índice ${ }_{G}$ em parâmetros de grafos vale para a omissão do índice $D$ em parâmetros de grafos orientados.

Se $a \in A$ e $\psi(a)=(u, v)$, dizemos que o arco $a$ vai de $u$ a $v$, e que $u$ é a ponta inicial de $a$ e $v$ é a ponta final de $a$. As pontas inicial e final de um arco também são chamadas simplesmente de suas pontas. Dizemos ainda que o arco $a$ incide ou é incidente em suas pontas.

Nos demais capítulos não vamos mais mencionar a função implícita $\psi$ e, a partir de agora, vamos abreviar o fato de que $\psi(a)=(u, v)$ escrevendo $a=(u, v)$ ou $a=u v$. Assim, quando nos referirmos a "um arco $u v$ ", seríamos mais precisos se disséssemos "um arco do tipo $u v$ ". A função implícita $\psi$ permite que existam diversos arcos com as mesmas pontas, e será conveniente enxergarmos cada arco como um par de vértices que é "rotulado" pelo "nome do arco".

Observe que podem existir arcos que têm um único vértice como ponta. Tais arcos são chamados de laços. Se dois arcos que não são laços têm a mesma ponta inicial e a mesma ponta final, dizemos que eles são paralelos.

No restante da seção, $D=(V, A)$ denota um grafo orientado.

Para cada arco $a=u v$, o reverso ou inverso de $a$ é um arco $a^{-1}:=v u$. Se $B \subseteq A$, denotamos $\left\{a^{-1}: a \in B\right\}$ por $B^{-1}$.

O grafo subjacente a $D$ é o grafo $G=(V, E)$ onde $E:=A$ e $\psi_{G}(a)=\{u, v\}$ se $\psi_{D}(a)=(u, v)$ para cada $a \in E$. Dizemos também que $D$ é uma orientação de $G$. Dizemos que $D$ é simples se seu grafo subjacente é simples. Nesse caso, a densidade de $D$ é a densidade de seu grafo subjacente. Um grafo orientado é uma árvore orientada se seu grafo subjacente é uma árvore. Um grafo orientado é um torneio se seu grafo subjacente é completo. Se $H$ é um grafo, dizemos que $H$ é um menor de $D$ se $H$ é um menor do grafo subjacente a $D$, e que $D$ é livre de $H$ como menor se seu grafo subjacente é livre de $H$ como menor.

Seja $D^{\prime}$ um grafo orientado. Dizemos que $D^{\prime}$ é um subgrafo de $D$ se $V_{D^{\prime}} \subseteq V_{D}, A_{D^{\prime}} \subseteq A_{D}$ e $\psi_{D^{\prime}}$ é a restrição de $\psi_{D}$ a $A_{D^{\prime}}$. Seja $S \subseteq V$. Denote por $A_{D}[S]$ o conjunto dos arcos de $D$ com as duas pontas em $S$. Dizemos que os arcos de $A_{D}[S]$ são induzidos por $S$. O subgrafo de $D$ induzido por $S$ é $D[S]:=\left(S, A_{D}[S]\right)$. Denotamos por $D-S$ o subgrafo $D[V \backslash S]$. Se 
$B \subseteq A$, denotamos $D-B:=(V, A \backslash B)$. Se $a$ é um arco, abreviamos $D+a:=(V, A \cup\{a\})$.

Seja $S \subseteq V$. Um arco entra em $S$ se tem sua ponta inicial fora de $S$ e sua ponta final em $S$. Uma arco sai de $S$ se seu reverso entra em $S$. Seja $B \subseteq A$. Denotamos por $\vec{\delta}_{B}(S)$ o conjunto dos arcos de $B$ que entram em $S$ e por $\overleftarrow{\delta}_{B}(S)$ o conjunto dos arcos de $B$ que saem de $S$. Abreviamos ainda $\overrightarrow{\delta_{D}}(S):=\vec{\delta}_{A}(S)$ e $\overleftarrow{\delta}_{D}(S):=\overleftarrow{\delta}_{A}(S)$ Abreviamos ainda $\vec{d}_{B}(S):=\left|\overrightarrow{\delta_{B}}(S)\right|$, $\overleftarrow{d}_{B}(S):=\left|\overleftarrow{\delta}_{B}(S)\right|$ e as variantes análogas dessas notações. Uma simples contagem mostra que, para $B \subseteq A$ fixo,

$$
\text { as funções } \vec{d}_{B}(S) \text { e } \overleftarrow{d}_{B}(S) \text { são submodulares. }
$$

Seja $v \in V$. Dizemos que $v$ é uma fonte se $\vec{\delta}(v)=\varnothing$ e que $v$ é um sorvedouro se $\overleftarrow{\delta}(v)=\varnothing$.

Seja $S \subseteq V$ um subconjunto não-vazio. A contração de $S$ consiste na operação que transforma o grafo orientado $D$ no grafo orientado $D^{\prime}=\left(V^{\prime}, A\right)$ definido da seguinte maneira. Tome $V^{\prime}:=(V \backslash S) \cup\{S\}$. Altere a função $\psi_{D}$ da seguinte maneira para obter $\psi_{D^{\prime}}$. Seja $a=u v \in A$. Se $u, v \in S$, tome $\psi_{D^{\prime}}(a):=(S, S)$. Se $u \in S$ e $v \notin S$, tome $\psi_{D^{\prime}}(a):=(S, v)$. Se $u \notin S$ e $v \in S$, tome $\psi_{D^{\prime}}(a):=(u, S)$. Para os demais arcos $a \in A$, tome $\psi_{D^{\prime}}(a):=\psi_{D}(a)$. Denotamos $D / S:=D^{\prime}$.

Seja $a=u v \in A$ um arco que não é um laço. A contração do arco $a$ consiste na operação que transforma $D$ no grafo orientado $(D-a) /\{u, v\}$.

Um pseudocaminho em $D$ é uma seqüência $P=\left\langle v_{0}, a_{1}, v_{1}, \ldots, a_{k}, v_{k}\right\rangle$ tal que $v_{0}, \ldots, v_{k}$ são vértices distintos de $D$ e $a_{i} \in A$ tem pontas $v_{i-1}$ e $v_{i}$ para $i=1, \ldots, k$. Dizemos que $v_{0}$ é a extremidade inicial de $P$ e que $v_{k}$ é a extremidade final de $P$, que $v_{0}$ e $v_{k}$ são as extremidades de $P$, que $P$ vai de $v_{0}$ a $v_{k}$ e que $P$ liga $v_{0}$ a $v_{k}$ em $D$. O comprimento de $P$ é $k$. Abreviamos $V_{P}:=\left\{v_{0}, \ldots, v_{k}\right\}$ e $A_{P}:=\left\{a_{1}, \ldots, a_{k}\right\}$. Um arco $a_{i}$ é um arco direto de $P$ se $a_{i}=v_{i-1} v_{i}$ e é um arco reverso de $P$ se $a_{i}=v_{i} v_{i-1}$. Algumas vezes confundimos propositalmente o pseudocaminho $P$ com o grafo $\left(V_{P}, A_{P}\right)$ ou com o conjunto $A_{P}$.

Um pseudocaminho $P=\left\langle v_{0}, a_{1}, v_{1}, \ldots, a_{k}, v_{k}\right\rangle$ em $D$ é um caminho se não possui arcos reversos. Nesse caso, dizemos que $v_{k}$ é acessível a partir de $v_{0}$ em $D$, que $v_{0}$ acessa $v_{k}$ em $D$ e que $P$ é um $\left(v_{0}, v_{k}\right)$-caminho. Algumas vezes abreviamos $P=v_{0} \cdots v_{k}$. Dizemos que $B \subseteq A$ é um $(r, s)$-corte se $B=\vec{\delta}(S)$ para algum subconjunto $S \subseteq V \backslash\{r\}$ tal que $s \in S$. Um arco $u v$ é transitivo em $D$ se $v$ é acessível a partir de $u$ em $D$.

Um pseudocircuito em $D$ é uma seqüência $C=\left\langle v_{0}, a_{1}, v_{1}, \ldots, a_{k}, v_{k}\right\rangle$ tal que $k \geq 1$, $v_{0}=v_{k},\left\langle v_{0}, a_{1}, v_{1}, \ldots, a_{k-1}, v_{k-1}\right\rangle$ é um pseudocaminho em $D$ e $a_{k} \in A$ tem pontas $v_{k-1}$ e $v_{k}$. Um arco $a_{i}$ é um arco direto de $C$ se $a_{i}=v_{i-1} v_{i}$ e é um arco reverso de $P$ se $a_{i}=v_{i} v_{i-1}$. O comprimento de $C$ é $k$. Abreviamos $V_{C}:=\left\{v_{0}, \ldots, v_{k-1}\right\}$ e $A_{C}:=\left\{a_{1}, \ldots, a_{k}\right\}$. Algumas vezes confundimos propositalmente o pseudocircuito $C$ com o grafo orientado $\left(V_{C}, A_{C}\right)$ ou com o conjunto $A_{C}$. Um pseudocircuito $C=\left\langle v_{0}, a_{1}, v_{1}, \ldots, a_{k}, v_{k}\right\rangle$ em $D$ é um circuito se não possui arcos reversos. Se $D$ não possui circuitos, dizemos que $D$ é acíclico.

Um componente forte de $D$ é um subgrafo $D^{\prime}$ de $D$ maximal tal que, para quaisquer $x, y \in V_{D^{\prime}}, x$ acessa $y$ em $D^{\prime}$. Dizemos que $D$ é fortemente conexo se possui um único componente forte. Um componente fraco de $D$ é um subgrafo de $D$ cujo grafo subjacente é um componente do grafo subjacente a $D$. Dizemos que $D$ é fracamente conexo se possui um único componente fraco. Dizemos que $D$ é fonte-sorvedouro-conexo se, para quaisquer componentes fortes $R$ e $S$ de $D$ tais que $\vec{\delta}(R)=\varnothing$ e $\overleftarrow{\delta}(S)=\varnothing$, existe um vértice de $S$ que é 
acessível a partir de algum vértice de $R$.

Dizemos que $B \subseteq A$ é uma ramificação de $D$ se o grafo subjacente a $(V, B)$ é acíclico e cada vértice $v \in V$ é ponta final de no máximo um arco de $B$. Seja $R$ o conjunto dos vértices que não são ponta final de nenhum arco de $B$. Cada elemento de $R$ é uma raiz de $B$. Dizemos que $R$ é o conjunto de raízes de $B$ e chamamos $B$ de $R$-ramificação. Se $R=\{r\}$, dizemos que $B$ é uma arborescência ou $r$-arborescência de $D$.

Dizemos que $J \subseteq A$ é uma junção de $D$ se o grafo orientado $\left(V, A \cup J^{-1}\right)$ é fortemente conexo. Dizemos que $B \subseteq A$ é um corte orientado de $D$ se $B=\vec{\delta}(S)$ para algum subconjunto não-vazio $S \subsetneq V$ tal que $\overleftarrow{\delta}(S)=\varnothing$. É claro que um subconjunto de arcos é uma junção se, e somente se, intersecta todos os cortes orientados.

A matriz de incidência de $D$ é a matriz $M$ sobre $V \times A$ definida da seguinte forma. Para cada vértice $v \in V$ e cada arco $a \in A$, defina

$$
M[v, a]:=\left\{\begin{aligned}
-1, & \text { se } v \text { é a ponta inicial de } a \\
+1, & \text { se } v \text { é a ponta final de } a \\
0, & \text { se } v \text { não é ponta de } a .
\end{aligned}\right.
$$

Dizemos que $D$ é planar se seu grafo subjacente $G$ é planar. Dada uma imersão de $D$ no plano, o grafo dual de $D$ (com relação a essa imersão) é a seguinte orientação de $G^{*}$ : se $e \in E_{G}$ corresponde a um arco $a=u v$ em $D$ e, percorrendo-se o arco $a$ de $u$ a $v$ na imersão, a face que se mantém à esquerda é $F$ e a face que se mantém à direita é $F^{\prime}$, então oriente $e^{*}$ de $F$ a $F^{\prime}$ para obter o $\operatorname{arco} a^{*}$. Denotamos essa orientação de $D^{*}$. Note que $A_{D^{*}}=\left\{a^{*}: a \in A\right\}$.

\section{Proposição 1.1 (Dualidade entre circuitos e cortes orientados)}

Seja $D=(V, A)$ um grafo orientado planar e $D^{*}$ seu dual. Então $C \subseteq A$ é um circuito em $D$ se, e somente se, o conjunto $\left\{a^{*}: a \in C\right\}$ é um corte orientado minimal em $D^{*}$.

\subsection{Hipergrafos}

Um hipergrafo é um par $\mathcal{H}=(V, \mathcal{E})$, onde $V$ é um conjunto e $\mathcal{E}$ é uma coleção de subconjuntos de $V$. Os elementos de $V$ são chamados de vértices, e os de $\mathcal{E}$, de hiper-arestas. Dizemos que $\mathcal{H}$ é um hipergrafo sobre $V$. Dizemos que $\mathcal{E}$ é o conjunto de hiper-arestas de $\mathcal{H}$. Algumas vezes denotamos o conjunto de hiper-arestas de $\mathcal{H}$ por $\mathcal{E}_{\mathcal{H}}$.

Um componente de $\mathcal{H}$ é um subconjunto $S \subseteq V$ maximal tal que $E \subseteq S$ ou $E \subseteq V \backslash S$ para toda hiper-aresta $E \in \mathcal{E}$. Dizemos que $\mathcal{H}$ é conexo se possui um único componente.

Um hipergrafo $\mathcal{C}=(V, \mathcal{E})$ é um clutter se, para quaisquer $E, F \in \mathcal{E}$ distintas, vale que $E \nsubseteq F$ e $E \nsupseteq F$. Nesse caso, definimos o bloqueador como o clutter $\mathcal{C}^{\prime}:=\left(V, \mathcal{E}^{\prime}\right)$, onde $\mathcal{E}^{\prime}$ é a coleção dos conjuntos minimais de $\left\{E^{\prime} \subseteq V: E^{\prime} \cap E \neq \varnothing\right.$ para todo $\left.E \in \mathcal{E}\right\}$. 


\subsection{Programação linear e inteira}

Sejam $x, x^{\prime} \in \mathbb{R}^{V}$. Escrevemos $x \leq x^{\prime}$ se $x_{v} \leq x_{v}^{\prime}$ para cada $v \in V$. Em alguns casos excepcionais permitimos que um componente de $x$ seja $\pm \infty$. É evidente que $-\infty \leq r \leq \infty$ para todo $r \in \mathbb{R} \cup\{ \pm \infty\}$.

Quando escrevemos $A x \leq b, A x=b$, etc., assumimos implicitamente a compatibilidade de dimensões entre a matriz $A$ e os vetores $x$ e $b$. Dizemos que $A x \leq b$ é um sistema de inequações lineares. Escrevemos 0 para indicar o vetor identicamente nulo indexado por um conjunto apropriado. Similarmente, escrevemos 1 para indicar o vetor que tem todas as componentes iguais a 1 e $\infty$ para indicar o vetor que tem todas as componentes iguais a $\infty$.

Um subconjunto $P$ de $\mathbb{R}^{V}$ é um poliedro se $P=\{x: A x \leq b\}$ para algum sistema de inequações lineares $A x \leq b$. Um vetor $x$ de $P$ é um vértice de $P$ se, para quaisquer $y, z \in P$ distintos e $\lambda \in \mathbb{R}$ com $0<\lambda<1$, vale que $x \neq \lambda y+(1-\lambda) z$. Um poliedro é integral se todos seus vértices são integrais.

Um problema de programação linear ou programa linear consiste em minimizar ou maximizar uma função linear sobre um poliedro. Por exemplo, dados vetores $c, b$ e uma matriz $A$, calcule

$$
\max \{c x: x \in P\},
$$

onde $P$ é o poliedro $\{x: A x \leq b\}$. Note que o valor de (1.6) pode ser $\pm \infty$. Os vetores de $P$ são chamados de viáveis no programa linear (1.6). Um vetor de $P$ é solução ótima do programa linear (1.6) se atinge o máximo em (1.6). Dizemos ainda que o sistema $A x \leq b$ determina o poliedro $P$.

Um dos principais resultados da teoria de poliedros bloqueadores e anti-bloqueadores é o seguinte teorema de FulKerson [1971]:

Teorema 1.2

Seja $\mathcal{C}=(V, \mathcal{E})$ um clutter e $\mathcal{C}^{\prime}=\left(V, \mathcal{E}^{\prime}\right)$ seu bloqueador. Então o sistema

$$
\begin{array}{ll}
x_{v} \geq 0 & \text { para todo } v \in V, \\
x(E) \geq 1 & \text { para todo } E \in \mathcal{E},
\end{array}
$$

determina um poliedro integral se, e somente se, o sistema

$$
\begin{array}{ll}
x_{v} \geq 0 & \text { para todo } v \in V, \\
x\left(E^{\prime}\right) \geq 1 & \text { para todo } E^{\prime} \in \mathcal{E}^{\prime},
\end{array}
$$

também determina um poliedro integral.

Teorema 1.3 (Teorema da dualidade de programação linear)

Seja $A$ uma matriz e sejam $b$ e $c$ vetores. Então

$$
\max \{c x: A x \leq b\}=\min \{y b: y \geq \mathbf{0}, y A=c\} .
$$

Na equação (1.9), o problema de minimização é chamado de programa linear dual do problema de maximização, que por sua vez é chamado de programa linear primal. 


\section{Teorema 1.4 (Folgas complementares)}

Sejam $x$ e $y$ vetores. As seguintes afirmações são equivalentes:

(i) $x$ e $y$ atingem o máximo e o mínimo, respectivamente, da equação (1.9);

(ii) se um componente de $y$ é positivo, então a respectiva inequação em $A x \leq b$ é satisfeira por $x$ com igualdade, ou seja, $y(b-A x)=0$.

\section{Teorema 1.5}

Seja $A$ uma matriz totalmente unimodular. Então, para qualquer vetor integral $b$, o poliedro $\{x: A x \leq b\}$ é integral.

\section{Teorema 1.6}

Suponha que o programa linear

$$
\max \{c x: x \geq \mathbf{0}, A x \leq b\}
$$

tem uma solução ótima $x^{*}$ tal que as colunas de $A$ correspondentes aos componentes positivos de $x^{*}$ formam uma matriz totalmente unimodular. Se o vetor $b$ é integral, então o programa linear (1.10) tem uma solução ótima integral.

Demonstração. Sejam $A^{\prime}$ e $c^{\prime}$ as partes de $A$ e $c$ correspondentes aos componentes positivos de $x^{*}$. É claro que

$$
\max \{c x: x \geq \mathbf{0}, A x \leq b\}=\max \left\{c^{\prime} x^{\prime}: x^{\prime} \geq \mathbf{0}, A^{\prime} x^{\prime} \leq b\right\}
$$

Como $A^{\prime}$ é totalmente unimodular e $b$ é integral, então pelo teorema 1.5 o lado direito de (1.11) possui uma solução ótima integral $x^{\prime *}$. Completando esse vetor $x^{\prime *}$ com componentes nulos, obtemos uma solução ótima integral para o lado esquerdo de (1.11).

Um sistema de inequações lineares $A x \leq b$ é totalmente dual integral se, para todo vetor integral $c$, o programa linear dual de $\max \{c x: A x \leq b\}$ admite uma solução ótima integral sempre que admite alguma solução ótima.

O seguinte resultado foi provado por EDMONDS E GILES [1977]:

\section{Teorema 1.7}

Seja $A x \leq b$ um sistema de inequações lineares. Se $A x \leq b$ é totalmente dual integral e $b$ e $c$ são vetores integrais, então ambos os programas lineares da equação de dualidade

$$
\max \{c x: A x \leq b\}=\min \{y b: y \geq \mathbf{0}, y A=c\}
$$

admitem soluções ótimas integrais sempre que algum deles admite alguma solução ótima.

Um sistema de inequações lineares $A x \leq b$ é box-TDI se, para quaisquer vetores $\ell$ e $u$ tais que $\ell \leq u$, o sistema $A x \leq b, \ell \leq x \leq u$ é totalmente dual integral. 
SCHRIJVER [1986] observou o seguinte:

\section{Proposição 1.8}

Seja $A x \leq b$ um sistema de inequações lineares. Se $A x \leq b$ é box-TDI, então o sistema $A x \leq b, \ell \leq x \leq u$ é totalmente dual integral para quaisquer vetores $\ell$ e $u$ tais que $\ell \leq u$, mesmo que alguns componentes de $\ell$ ou $u$ sejam $\pm \infty$.

\section{Teorema 1.9}

Seja $A x \leq b$ um sistema de inequações lineares. Suponha que, para todo vetor $c$ para o qual

$$
\max \{c x: A x \leq b\}
$$

é finito, o programa linear dual de (1.13) tem uma solução ótima $y^{*}$ tal que as linhas de $A$ correspondentes aos componentes positivos de $y^{*}$ formam uma matriz totalmente unimodular. Então o sistema $A x \leq b$ é box-TDI.

Demonstração. Sejam $\ell$ e $u$ vetores tais que $\ell \leq u$. Seja $c$ um vetor integral. O problema dual de $\max \{c x: A x \leq b, \ell \leq x \leq u\}$ é

$$
\min \{y b-w \ell+z u: y, w, z \geq \mathbf{0}, y A-w+z=c\} .
$$

Seja $y^{*}, w^{*}, z^{*}$ uma solução ótima de (1.14). Tome $c^{\prime}:=c-z^{*}+w^{*}$. A hipótese do teorema nos garante que o programa linear $\min \left\{y^{\prime} b: y^{\prime} \geq \mathbf{0}, y^{\prime} A=c^{\prime}\right\}$ tem uma solução ótima $y^{\prime *}$ tal que as linhas de $A$ correspondentes aos componentes positivos de $y^{\prime *}$ formam uma matriz totalmente unimodular. Além disso, pela escolha do vetor $c^{\prime}$, temos que $y^{\prime *}, w^{*}, z^{*}$ é uma solução ótima de (1.14). Como as linhas do sistema $A x \leq b, \ell \leq x \leq u$ correspondentes aos componentes positivos de $y^{\prime *}, w^{*}, z^{*}$ formam uma matriz totalmente unimodular, concluímos do teorema 1.6 que existe uma solução ótima integral para (1.14).

Mencionamos finalmente um resultado de Cook, Fonlupt e SchriJver [1986]. A prova desse resultado é bastante curta e acessível.

\section{Teorema 1.10}

Seja $A x \leq b$ um sistema de inequações lineares totalmente dual integral, onde $x$ é indexado por um conjunto de tamanho $n$. Então, para qualquer vetor integral $c$, o programa linear $\min \{c x: y \geq \mathbf{0}, y A=c\}$ possui uma solução ótima integral cujo suporte tem tamanho no máximo $2 n-1$. 


\subsection{Matróides}

Um matróide é um par $M=(E, \mathcal{I})$, onde $E$ é um conjunto e $\mathcal{I}$ é uma coleção de subconjuntos de $E$, que satisfaz as seguintes condições:

(i) $\varnothing \in \mathcal{I}$,

(ii) se $I \in \mathcal{I}$ e $I^{\prime} \subseteq I$, então $I^{\prime} \in \mathcal{I}$,

(iii) se $I, I^{\prime} \in \mathcal{I}$ e $|I|<\left|I^{\prime}\right|$, então existe $e \in I^{\prime} \backslash I$ tal que $I \cup\{e\} \in \mathcal{I}$.

Dizemos que $M$ é um matróide sobre $E$ e que $E$ é o conjunto-base do matróide $M$. Chamamos os membros de $\mathcal{I}$ de conjuntos independentes de $M$. Um subconjunto de $E$ que não está em $\mathcal{I}$ é um conjunto dependente de $M$.

Uma base de $M$ é um conjunto independente maximal. Seja $B$ uma base de $M$ e sejam $x, y \in E$. Algumas vezes será conveniente escrevermos " $B-x+y$ " no lugar de " $(B \backslash\{x\}) \cup\{y\}$ ". Vamos precisar da seguinte caracterização baseada em propriedades de troca:

\section{Teorema 1.11 (Caracterização de coleção de bases de um matróide)}

Seja $E$ um conjunto finito. Seja $\mathcal{B}$ uma coleção de subconjuntos de $E$ satisfazendo as seguintes condições:

(i) $\mathcal{B} \neq \varnothing \mathrm{e}$

(ii) para quaisquer $B_{1}, B_{2} \in \mathcal{B}$ e $e \in B_{1} \backslash B_{2}$, existe $f \in B_{2} \backslash B_{1}$ tal que $B_{1}-e+f \in \mathcal{B}$.

Seja $\mathcal{I}$ a coleção dos subconjuntos de $E$ que estão contidos em algum membro de $\mathcal{B}$. Então $(E, \mathcal{I})$ é um matróide que tem $\mathcal{B}$ como coleção de bases.

Demonstração. Como $\mathcal{B}$ satisfaz (1.16)(i), então $\mathcal{I}$ satisfaz (1.15)(i). Além disso, é evidente que $\mathcal{I}$ satisfaz (1.15)(ii). Resta provarmos que $\mathcal{I}$ satisfaz (1.15)(iii). Suponha que existem $I, I^{\prime} \in \mathcal{I}$ tais que $|I|<\left|I^{\prime}\right|$ e, para todo $e \in I^{\prime} \backslash I$, temos que $I \cup\{e\} \notin \mathcal{I}$. Vamos ver que isso é impossível.

Primeiro vamos mostrar que

$$
|B|=\left|B^{\prime}\right| \text { para quaisquer } B, B^{\prime} \in \mathcal{B} \text {. }
$$

Pois suponha o contrário. Dentre todos os pares $\left(B, B^{\prime}\right) \in \mathcal{B} \times \mathcal{B}$ tais que $|B|>\left|B^{\prime}\right|$, escolha um que minimiza $\left|B \backslash B^{\prime}\right|$. É óbvio que $B \backslash B^{\prime} \neq \varnothing$. Seja $e \in B \backslash B^{\prime}$. Por (1.16)(ii), existe $f \in B^{\prime} \backslash B$ tal que $B^{\prime \prime}:=B-e+f \in \mathcal{B}$. É evidente que $\left|B^{\prime \prime}\right|=|B|>\left|B^{\prime}\right|$ e que $\left|B^{\prime \prime} \backslash B^{\prime}\right|<\left|B \backslash B^{\prime}\right|$, contradizendo a escolha de $B$ e $B^{\prime}$. Essa contradição finaliza a prova de (1.17).

Dentre todos os pares $\left(B, B^{\prime}\right) \in \mathcal{B} \times \mathcal{B}$ tais que $I \subseteq B$ e $I^{\prime} \subseteq B^{\prime}$, escolha um que minimiza $\left|B^{\prime} \backslash\left(I^{\prime} \cup B\right)\right|$. Observe que, pela escolha de $I$ e $I^{\prime}$,

$$
I^{\prime} \backslash B=I^{\prime} \backslash I
$$


Vamos mostrar agora que

$$
B^{\prime} \backslash B=I^{\prime} \backslash B
$$

É óbvio que $B^{\prime} \backslash B \supseteq I^{\prime} \backslash B$. Suponha então que $B^{\prime} \backslash B \supsetneq I^{\prime} \backslash B$, ou seja, suponha que existe $e \in B^{\prime} \backslash\left(I^{\prime} \cup B\right)$. Por $(1.16)$ (ii), existe $f \in B \backslash B^{\prime}$ tal que $B^{\prime \prime}:=B^{\prime}-e+f \in \mathcal{B}$. Mas $I^{\prime} \subseteq B^{\prime \prime}$ e $\left|B^{\prime \prime} \backslash\left(I^{\prime} \cup B\right)\right|<\left|B^{\prime} \backslash\left(I^{\prime} \cup B\right)\right|$, contradizendo a escolha de $B^{\prime}$. Essa contradição conclui a prova de (1.19).

Agora vamos mostrar que

$$
B \subseteq I \cup B^{\prime}
$$

Pois suponha que existe $e \in B \backslash\left(I \cup B^{\prime}\right)$. Pela condição (1.16)(ii), existe $f \in B^{\prime} \backslash B=I^{\prime} \backslash B$ tal que $B^{\prime \prime}:=B-e+f \in \mathcal{B}$. Mas então $I \cup\{f\} \subseteq B^{\prime \prime} \in \mathcal{B}$, de modo que $I \cup\{f\} \in \mathcal{I}$. Essa contradição conclui a prova de (1.20).

Segue de (1.20) que $B \backslash B^{\prime}=I \backslash B^{\prime}$. Como $B^{\prime} \supseteq I^{\prime}$, então $I \backslash B^{\prime} \subseteq I \backslash I^{\prime}$. Concluímos que

$$
B \backslash B^{\prime} \subseteq I \backslash I^{\prime}
$$

Por (1.18) e (1.19), temos que

$$
B^{\prime} \backslash B=I^{\prime} \backslash I \text {. }
$$

Por outro lado, sabemos de (1.17) que $|B|=\left|B^{\prime}\right|$, de modo que $\left|B \backslash B^{\prime}\right|=\left|B^{\prime} \backslash B\right|$. Mas então, por (1.21) e (1.22), temos que $\left|I \backslash I^{\prime}\right| \geq\left|B \backslash B^{\prime}\right|=\left|B^{\prime} \backslash B\right|=\left|I^{\prime} \backslash I\right|$, de modo que $|I| \geq\left|I^{\prime}\right|$. Essa contradição finaliza a prova de que $\mathcal{I}$ satisfaz (1.15)(iii), e portanto do teorema.

Seja $M=(E, \mathcal{I})$ um matróide e $F \subseteq E$. Uma base de $F$ é um subconjunto de $F$ que é independente maximal. É evidente que todas as bases de $F$ têm o mesmo tamanho. Esse tamanho é chamado de posto de $F$. Seja $r_{M}: \mathcal{P}(E) \rightarrow \mathbb{R}$ a função que associa a cada $F \subseteq E$ o posto de $F$. A função $r_{M}$ é a função posto de $M$.

\section{Proposição 1.12 (Submodularidade da função posto de matróides)}

A função posto de todo matróide é submodular.

Demonstração. Seja $M=(E, \mathcal{I})$ um matróide.

Sejam $U, W \subseteq E$. Seja $B_{U \cap W}$ uma base de $U \cap W$. É fácil ver que $B_{U \cap W}$ está contido numa base de $U \cup W$. Seja $B_{U \cup W}$ uma tal base. Note que $B_{U \cup W} \cap U$ e $B_{U \cup W} \cap W$ são independentes, de modo que

$$
\begin{aligned}
r_{M}(U)+r_{M}(W) & \geq\left|B_{U \cup W} \cap U\right|+\left|B_{U \cup W} \cap W\right| \\
& =\left|\left(B_{U \cup W} \cap U\right) \cup\left(B_{U \cup W} \cap W\right)\right|+\left|\left(B_{U \cup W} \cap U\right) \cap\left(B_{U \cup W} \cap W\right)\right| \\
& =\left|B_{U \cup W} \cap(U \cup W)\right|+\left|B_{U \cup W} \cap(U \cap W)\right| .
\end{aligned}
$$

Mas como $B_{U \cup W} \cap(U \cup W)=B_{U \cup W}$ e $B_{U \cup W} \cap(U \cap W)=B_{U \cap W}$, então

$$
\begin{aligned}
r_{M}(U)+r_{M}(W) & \geq\left|B_{U \cup W} \cap(U \cup W)\right|+\left|B_{U \cup W} \cap(U \cap W)\right| \\
& =\left|B_{U \cup W}\right|+\left|B_{U \cap W}\right|=r_{M}(U \cup W)+r_{M}(U \cap W),
\end{aligned}
$$

como queríamos. 


\section{Corolário 1.12.1 (Submodularidade da função posto de matróides gráficos)}

Seja $G=(V, E)$ um grafo. Defina a função $r: \mathcal{P}(E) \rightarrow \mathbb{R}$ como $r(F):=|V|-c(V, F)$, onde $c(V, F)$ denota o número de componentes do grafo $(V, F)$. Então a função $r$ é submodular.

Demonstração. Defina $\mathcal{I}$ como a coleção de todos os subconjuntos de arestas $F \subseteq E$ tais que $(V, F)$ é uma floresta. Seja $\mathcal{B}$ a coleção dos membros maximais de $\mathcal{I}$. É fácil ver que $\mathcal{B}$ satisfaz as condições (1.16). Pelo teorema $1.11, M:=(E, \mathcal{I})$ é um matróide. É fácil ver que a função $r$ é igual à função posto de $M$. Agora o resultado segue da proposição 1.12.

Seja $M=(E, \mathcal{I})$ um matróide. Um circuito de $M$ é um conjunto dependente minimal. Se $\{e\}$ é um circuito para algum $e \in E$, dizemos que é um laço de $M$. Seja $E^{\prime}$ o conjunto de todos os elementos de $E$ que não são laços. Se $\{e, f\}$ é um circuito para certos $e, f \in E^{\prime}$ distintos, dizemos que $e$ e $f$ são paralelos.

\section{Proposição 1.13}

Seja $M=(E, \mathcal{I})$ um matróide e sejam $e, f, g \in E$. Se $e$ e $f$ são paralelos e $f$ e $g$ são paralelos, então $e$ e $g$ são paralelos.

Demonstração. É claro que $r_{M}(\{e, f\})=r_{M}(\{f, g\})=r_{M}(\{e\})=r_{M}(\{f\})=r_{M}(\{g\})=1$. Pela proposição 1.12, temos que $r_{M}(\{f\})+r_{M}(\{e, f, g\}) \leq r_{M}(\{e, f\})=r_{M}(\{f, g\})$. Logo, $r_{M}(\{e, f, g\}) \leq 1$. Então qualquer subconjunto de $\{e, f, g\}$ com dois elementos é dependente minimal. Em particular, $\{e, g\}$ é um circuito, como queríamos. 


\section{Capítulo 2}

\section{Fluxos submodulares}

Edmonds e Giles desenvolveram um poderoso arcabouço poliédrico envolvendo o conceito de fluxo submodular, generalizando e unificando vários resultados importantes em otimização combinatória. Esse arcabouço é o mais conhecido de diversos modelos propostos com o mesmo intuito e baseados em idéias muito semelhantes. Neste capítulo, apresentamos o teorema de Edmonds-Giles sobre fluxos submodulares e uma variante desse teorema devida a Schrijver, a partir dos quais derivamos várias relações min-max.

O teorema de Edmonds-Giles afirma que um certo sistema de inequações lineares $A x \leq b$ é box-TDI. Nossa estratégia para prová-lo será usar o teorema 1.9: é suficiente encontrarmos uma solução ótima $y^{*}$ para o programa linear dual de $\max \{c x: A x \leq b\}$ tal que a submatriz de $A$ correspondente ao suporte de $y^{*}$ é totalmente unimodular.

A principal idéia da demonstração será escolher uma solução $y^{*}$ cujo suporte seja algo que definiremos como uma família livre de cruzamentos. Tais famílias podem ser representadas através de uma árvore e, a partir dessa representação, poderemos mostrar que a submatriz em questão é uma matriz de rede, um tipo notável de matriz totalmente unimodular. Assim, o teorema estará provado.

Essa estratégia de escolher uma solução livre de cruzamentos é muitas vezes chamada de técnica do descruzamento (uncrossing) e tem se mostrado muito poderosa na resolução de diversos problemas de otimização combinatória. Essa técnica está intimamente relacionada com funções submodulares, que têm um papel fundamental em otimização combinatória, como será visto ao longo deste texto.

O restante do capítulo está organizado da seguinte maneira: na seção 2.1, definimos matrizes de rede e provamos que estas são totalmente unimodulares; na seção 2.2 , definimos famílias livres de cruzamentos, mostramos que elas podem ser representadas através de árvores e, a partir dessa representação, construímos uma matriz de rede; na seção 2.3, combinamos os resultados das seções anteriores para provar o teorema de Edmonds-Giles, a partir do qual obtemos o teorema de Lucchesi-Younger e o teorema de intersecção de matróides de Edmonds; finalmente, na seção 2.4, mostramos uma variante devida a Schrijver, do qual derivamos mais relações min-max. 


\subsection{Matrizes de rede}

Seja $T=\left(V, A_{T}\right)$ uma árvore orientada e $D=\left(V, A_{D}\right)$ um grafo orientado. A matriz de rede gerada por $T$ e $D$ é a matriz $N$ sobre $A_{T} \times A_{D}$ definida da seguinte maneira. Para cada par de $\operatorname{arcos} a_{T} \in A_{T}$ e $a_{D}=u v \in A_{D}$, denote por $P$ o pseudocaminho que liga $u$ a $v$ em $T$, e defina

$$
N\left[a_{T}, a_{D}\right]:=\left\{\begin{aligned}
+1, & \text { se } a_{T} \text { é um arco direto de } P, \\
-1, & \text { se } a_{T} \text { é um arco reverso de } P, \\
0, & \text { se } a_{T} \text { não é um arco de } P .
\end{aligned}\right.
$$

Vejamos alguns exemplos de matrizes de rede.

Seja $D=(V, A)$ um grafo orientado. Tome $V^{\prime}:=V \cup\{z\}$, onde $z$ é um vértice novo. Defina a árvore orientada $T:=\left(V^{\prime}, A_{T}\right)$, onde $A_{T}:=\{z v: v \in V\}$. É fácil ver que a matriz de rede gerada por $T=\left(V^{\prime}, A_{T}\right)$ e $D^{\prime}:=\left(V^{\prime}, A\right)$ é igual à matriz de incidência de $D$, identificando cada arco $z v \in A_{T}$ com o vértice $v \in V$.

Seja $G=(V, E)$ um grafo $(U, W)$-bipartido. Tome $V^{\prime}:=V \cup\{z\}$, onde $z$ é um vértice novo. Defina a árvore orientada $T:=\left(V^{\prime}, A_{T}\right)$ onde $A_{T}:=\{u z: u \in U\} \cup\{z w: w \in W\}$. Oriente todas as arestas do grafo $\left(V^{\prime}, E\right)$ de $U$ para $W$, obtendo o grafo orientado $D$. É fácil ver que a matriz de rede gerada por $T$ e $D$ é igual à matriz de incidência de $G$, identificando cada arco $u z \in A_{T}$ com o vértice $u \in U$ e cada arco $z w \in A_{T}$ com o vértice $w \in W$.

No restante desta seção vamos provar que matrizes de rede são totalmente unimodulares.

\section{Lema $2.1 \alpha$ (Hereditariedade de matrizes de rede)}

Submatrizes de uma matriz de rede também são matrizes de rede.

Demonstração. Seja $N$ uma matriz de rede gerada por $T=\left(V, A_{T}\right)$ e $D=\left(V, A_{D}\right)$. É fácil ver que remover de $N$ a coluna $a_{D} \in A_{D}$ corresponde a remover de $D$ o arco $a_{D}$. Similarmente, remover de $N$ a linha $a_{T}=u v \in A_{T}$ corresponde a contrair em $T$ o $\operatorname{arco} a_{T}$ e identificar em $D$ os vértices $u$ e $v$.

O principal resultado desta seção foi provado implicitamente por TUTTE [1965]:

\section{Teorema 2.1 (Unimodularidade total de matrizes de rede)}

Toda matriz de rede é totalmente unimodular.

Demonstração. Pelo lema $2.1 \alpha$ da hereditariedade, basta provarmos que toda matriz de rede quadrada tem determinante $0,+1$ ou -1 .

Seja $N$ uma matriz de rede quadrada gerada por $T=\left(V, A_{T}\right)$ e $D=\left(V, A_{D}\right)$. A prova é por indução na ordem de $N$, tomando como base o caso trivial em que $N$ é uma matriz $1 \times 1$.

Suponha que $\operatorname{det}(N) \neq 0$. Nosso objetivo é transformar uma linha conveniente de $N$ numa linha contendo 0s e um único 1 . As operações sobre $N$ que aplicaremos nessa transformação mantêm $N$ como uma matriz de rede e preservam o valor absoluto do determinante. Assim, após a transformação, poderemos expandir o determinante de $N$ através dessa linha e usar o lema $2.1 \alpha$ da hereditariedade e a hipótese de indução para concluir que $|\operatorname{det}(N)|=1$. 
Seja $u$ um vértice que é ponta de um único arco de $A_{T}$. Chamemos esse arco de $a_{T}$. Primeiro vamos transformar a linha $a_{T}$ numa linha contendo somente 0 s e 1s. Para tanto, reorientamos os arcos de $T$ e de $D$ de modo que todo arco incidente em $u$ tenha $u$ como ponta inicial. Observe que trocar um arco $a_{D} \in A_{D}$ pelo seu reverso $a_{D}^{-1}$ corresponde a multiplicar a coluna $a_{D}$ por -1 , e analogamente para a reorientação de arcos de $A_{T}$.

Agora vamos deixar a linha $a_{T}$ com um único 1. Escolha dois 1s nessa linha, provenientes de colunas $u v, u w \in A_{D}$. Não é difícil ver que subtrair a coluna $u v$ da coluna $u w$ corresponde a trocar o arco uw pelo arco $v w$.

Podemos assim obter uma matriz de rede com determinante igual, em módulo, ao da matriz original, e que contém uma linha com 0s e um único 1. Ao expandirmos o determinante através dessa linha, obtemos do lema $2.1 \alpha$ da hereditariedade e da hipótese de indução que $|\operatorname{det}(N)|=1$.

Matrizes de rede têm um papel fundamental em otimização combinatória. Mais referências sobre elas podem ser vistas nas notas no final do capítulo.

\subsection{Famílias livres de cruzamentos e representações arbóreas}

Seja $V$ um conjunto. Dois subconjuntos $U, W \subseteq V$ se cruzam se

$$
U \cap W \neq \varnothing, \quad U \nsubseteq W, \quad U \nsupseteq W \quad \text { e } \quad U \cup W \neq V .
$$

Uma família de subconjuntos de $V$ é livre de cruzamentos se não possui dois conjuntos que se cruzam, e é laminar se não possui conjuntos $U$ e $W$ tais que

$$
U \cap W \neq \varnothing, \quad U \nsubseteq W \quad \text { e } \quad U \nsupseteq W .
$$

É fácil ver que as famílias laminares são exatamente as que podem ser representadas no plano por um diagrama de Venn em que as linhas não se cruzam. Já as livres de cruzamentos são as que admitem uma tal representação na esfera. Isso pode ser deduzido da seguinte observação facilmente verificável:

Seja $\mathcal{C}$ uma família de subconjuntos de $V$ livre de cruzamentos e $v \in V$. Então $\mathcal{C}_{v}:=\{V \backslash S: S \in \mathcal{C}$ e $v \in S\} \cup\{S: S \in \mathcal{C}$ e $v \notin S\}$ é laminar.

Vamos ver agora que as famílias livres de cruzamentos são exatamente as que podem ser, de certa forma, representadas por árvores. Tal representação nos permitirá definir uma matriz de rede a partir de uma família livre de cruzamentos associada a um grafo orientado.

Seja $V$ um conjunto, $T=\left(V_{T}, A_{T}\right)$ uma árvore orientada e $\pi: V \rightarrow V_{T}$ uma função. Para cada arco $a=u w \in A_{T}$, defina $S_{a}:=\left\{v \in V: \pi(v) \in T_{w}\right\}$, onde $T_{w}$ é o componente fraco de $T-a$ que contém o vértice $w$. Denote $\mathcal{C}_{T, \pi}:=\left\{S_{a}: a \in A_{T}\right\}$. Dizemos que o par $(T, \pi)$ é uma representação arbórea da família $\mathcal{C}_{T, \pi}$. Se $A_{T}$ é uma arborescência de $T$, então $(T, \pi)$ é uma representação arbórea enraizada da família $\mathcal{C}_{T, \pi}$. 
A seguinte caracterização foi provada por EDMOnds E GiLEs [1977]:

\section{Teorema 2.2 (Representação arbórea de famílias livres de cruzamentos)}

Uma família é livre de cruzamentos se, e somente se, admite uma representação arbórea; e é laminar se, e somente se, admite uma representação arbórea enraizada.

Demonstração. É claro que $\mathcal{C}_{T, \pi}$ é livre de cruzamentos. Também é óbvio que $\mathcal{C}_{T, \pi}$ é laminar se $A_{T}$ é uma arborescência de $T$. Primeiro vamos mostrar que toda família laminar $\mathcal{C}$ de subconjuntos de $V$ admite uma representação arbórea enraizada. Nossa prova é por indução em $|\mathcal{C}|$, tomando como base o caso trivial em que $\mathcal{C}=\varnothing$.

Escolha um conjunto minimal $S \in \mathcal{C}$ e tome $\mathcal{C}^{\prime}:=\mathcal{C} \backslash\{S\}$. Pela hipótese de indução, a família laminar $\mathcal{C}^{\prime}$ tem uma representação arbórea enraizada $\left(T^{\prime}, \pi^{\prime}\right)$. É fácil obter uma representação arbórea enraizada de $\mathcal{C}$ se $S=\varnothing$ : basta adicionar a $T^{\prime}$ um vértice novo $w$ e um arco novo $v w$, onde $v$ é qualquer vértice de $T^{\prime}$. Suponha então que $S \neq \varnothing$.

Afirmamos que existe um vértice $u$ de $T^{\prime}$ tal que $\pi^{\prime}(s)=u$ para todo $s \in S$. De fato, suponha que $\pi^{\prime}(s) \neq \pi^{\prime}(t)$ para certos $s, t \in S$. Podemos supor que o pseudocaminho que liga $\pi^{\prime}(s)$ a $\pi^{\prime}(t)$ em $T^{\prime}$ tem um arco direto $a$. Mas então, como $\mathcal{C}$ é laminar, temos $S_{a} \subseteq S \backslash\{s\}$, contradizendo a minimalidade de $S$.

Agora é fácil obter uma representação arbórea enraizada de $\mathcal{C}$ : basta adicionar a $T^{\prime}$ um vértice novo $w$ e o arco novo $u w$, e alterar $\pi^{\prime}$ de modo que $\pi^{\prime}(s)=w$ para todo $s \in S$.

Para construir uma representação arbórea de uma família livre de cruzamentos $\mathcal{C}$, proceda da seguinte forma: fixe $v \in V$ e tome a família laminar $\mathcal{C}_{v}$ como na afirmação (2.1). Construa uma representação arbórea enraizada $(T, \pi)$ de $\mathcal{C}_{v}$ e reoriente alguns arcos de $T$ para obter uma representação arbórea de $\mathcal{C}$.

Usando representações arbóreas, podemos derivar a unimodularidade total de certas matrizes definidas a partir de um grafo orientado e de uma família livre de cruzamentos associada.

\section{Corolário 2.2.1}

Seja $D=(V, A)$ um grafo orientado e $\mathcal{C}$ uma coleção de subconjuntos de $V$. Seja $N$ a matriz $\mathcal{C} \times A$ definida como

$$
N[S, a]:=\left\{\begin{aligned}
+1, & \text { se } a \in \vec{\delta}(S), \\
-1, & \text { se } a \in \overleftarrow{\delta}(S), \\
0, & \text { caso contrário, }
\end{aligned}\right.
$$

para cada $S \in \mathcal{C}$ e $a \in A$. Se $\mathcal{C}$ é livre de cruzamentos, então $N$ é totalmente unimodular.

Demonstração. Pelo teorema 2.2 , a coleção $\mathcal{C}$ admite uma representação arbórea. Seja $(T, \pi)$ uma tal representação. Defina o grafo orientado $D^{\prime}:=\left(V_{T}, A^{\prime}\right)$, onde

$$
A^{\prime}:=\{(\pi(u), \pi(v)):(u, v) \in A\} .
$$

Não é difícil ver que a matriz de rede gerada por $T$ e $D^{\prime}$ é igual a $N$, identificando cada arco $a \in A_{T}$ com o conjunto $S_{a} \in \mathcal{C}$ e cada arco $(\pi(u), \pi(v)) \in A^{\prime}$ com o arco $(u, v) \in A$. Então, pelo teorema 2.1, a matriz $N$ é totalmente unimodular. 


\subsection{O teorema de Edmonds-Giles}

EDMONDS E GILES [1977] provaram uma relação min-max bastante geral, que inclui como casos especiais o teorema de intersecção de polimatróides de Edmonds, o teorema de Lucchesi-Younger, o problema da circulação de custo mínimo e certos problemas de orientação de grafos e de aumento de conexidade. Nesta seção, apresentamos esse arcabouço poliédrico de Edmonds e Giles e dele derivamos duas relações min-max.

Seja $V$ um conjunto. Seja $\mathcal{C}$ uma família de subconjuntos de $V$ que não é livre de cruzamentos. Suponha que queremos transformar $\mathcal{C}$ numa família livre de cruzamentos repetindo a seguinte operação sempre que possível: se $U, W \in \mathcal{C}$ se cruzam, troque $U$ e $W$ por $U \cap W$ e $U \cup W$. O lema a seguir mostra que esse processo sempre termina, já que $\sum_{S \in \mathcal{C}}|S||V \backslash S| \geq 0$ para toda família $\mathcal{C}$.

Lema $2.3 \alpha$ (Lema do descruzamento)

Seja $V$ um conjunto e sejam $U, W \subseteq V$ tais que $U \nsubseteq W$ e $U \nsupseteq W$. Então

$$
|U||\bar{U}|+|W||\bar{W}|>|U \cap W||\overline{U \cap W}|+|U \cup W||\overline{U \cup W}|,
$$

onde $\bar{S}:=V \backslash S$ para todo $S \subseteq V$.

Demonstração. Abrevie $\alpha:=|U \cap W|, \beta:=|U \backslash W|, \gamma:=|W \backslash U|$ e $\delta:=|\overline{U \cup W}|$. Então

$$
\begin{aligned}
|U||\bar{U}|+|W||\bar{W}| & =(\alpha+\beta)(\gamma+\delta)+(\alpha+\gamma)(\beta+\delta) \\
& =2 \alpha \delta+\alpha \gamma+\beta \delta+\alpha \beta+\gamma \delta+2 \beta \gamma
\end{aligned}
$$

$\mathrm{e}$

$$
\begin{aligned}
|U \cap W||\overline{U \cap W}|+|U \cup W||\overline{U \cup W}| & =\alpha(\beta+\gamma+\delta)+(\alpha+\beta+\gamma) \delta \\
& =2 \alpha \delta+\alpha \gamma+\beta \delta+\alpha \beta+\gamma \delta .
\end{aligned}
$$

Agora o lema segue da desigualdade $\beta \gamma>0$.

Seja $V$ um conjunto e $\mathcal{C}$ uma coleção de subconjuntos de $V$. Dizemos que $\mathcal{C}$ é fechada sob cruzamentos se $U \cap W, U \cup W \in \mathcal{C}$ sempre que $U, W \in \mathcal{C}$ se cruzam.

Seja $\mathcal{C}$ uma coleção de subconjuntos de $V$ fechada sob cruzamentos. Uma função $f: \mathcal{C} \rightarrow \mathbb{R}$ é chamada submodular em cruzamentos se

$$
f(U \cap W)+f(U \cup W) \leq f(U)+f(W)
$$

sempre que $U, W \in \mathcal{C}$ se cruzam.

Podemos finalmente definir fluxos submodulares. Seja $D=(V, A)$ um grafo orientado, $\mathcal{C}$ uma coleção de subconjuntos de $V$ fechada sob cruzamentos e $f: \mathcal{C} \rightarrow \mathbb{R}$ uma função submodular em cruzamentos. Um fluxo submodular é uma função $x: A \rightarrow \mathbb{R}$ tal que

$$
x(\vec{\delta}(S))-x(\overleftarrow{\delta}(S)) \leq f(S) \quad \text { para todo } S \in \mathcal{C}
$$


EDMONDS E GILES [1977] provaram o seguinte resultado:

\section{Teorema 2.3 (Teorema de Edmonds-Giles)}

O sistema (2.2) é box-TDI.

Demonstração. Considere o programa linear

$$
\max \{c x: x \text { é um fluxo submodular }\} \text {. }
$$

Nossa estratégia será encontrar uma solução ótima $y^{*}$ para o programa linear dual de (2.3) tal que as linhas da matriz de (2.3) correspondentes aos componentes positivos de $y^{*}$ formem uma matriz totalmente unimodular. Feito isso, o resultado seguirá do teorema 1.9.

Note que a matriz de (2.3) tem o mesmo formato da matriz $N$ definida no corolário 2.2.1. Assim, se o suporte de $y^{*}$ for uma coleção livre de cruzamentos, a unimodularidade total da submatriz correspondente seguirá do corolário 2.2.1. Para obter uma tal solução dual $y^{*}$, utilizaremos o lema $2.3 \alpha$ do descruzamento.

O programa linear dual de (2.3) é

$$
\min \left\{y f: y \in \mathbb{R}_{\geq 0}^{\mathcal{C}}, \sum_{S \in \mathcal{C}} y_{S}\left(\chi^{\vec{\delta}(S)}-\chi^{\overleftarrow{\delta}(S)}\right)=c\right\}
$$

Dentre todas as soluções ótimas $y$ de (2.4), escolha como $y^{*}$ uma solução ótima que minimiza a função linear

$$
\phi(y):=\sum_{S \in \mathcal{C}} y_{S}|S||V \backslash S| .
$$

Vamos mostrar que a coleção $\mathcal{C}^{*}:=\left\{S \in \mathcal{C}: y_{S}^{*}>0\right\}$ é livre de cruzamentos. Suponha o contrário, e sejam $U, W \in \mathcal{C}^{*}$ conjuntos que se cruzam. Tome $\varepsilon:=\min \left\{y_{U}^{*}, y_{W}^{*}\right\}$ e defina

$$
y_{S}^{\prime}:= \begin{cases}y_{S}^{*}-\varepsilon, & \text { se } S=U \text { ou } S=W, \\ y_{S}^{*}+\varepsilon, & \text { se } S=U \cap W \text { ou } S=U \cup W \\ y_{S}^{*}, & \text { caso contrário, }\end{cases}
$$

para todo $S \in \mathcal{C}$. É fácil verificar que

$$
\chi^{\vec{\delta}(U \cap W)}-\chi^{\overleftarrow{\delta}(U \cap W)}+\chi^{\vec{\delta}(U \cup W)}-\chi^{\overleftarrow{\delta}(U \cup W)}=\chi^{\vec{\delta}(U)}-\chi^{\overleftarrow{\delta}(U)}+\chi^{\vec{\delta}(W)}-\chi^{\overleftarrow{\delta}(W)}
$$

de modo que $y^{\prime}$ é viável no programa linear (2.4). Além disso, como $f$ é submodular em cruzamentos, temos que $y^{\prime} f \leq y^{*} f$, ou seja, $y^{\prime}$ também é solução ótima de (2.4). Porém, pelo lema $2.3 \alpha$ do descruzamento, temos $\phi\left(y^{\prime}\right)<\phi\left(y^{*}\right)$, contradizendo a escolha de $y^{*}$.

Como $\mathcal{C}^{*}$ é livre de cruzamentos, o corolário 2.2.1 nos garante que as linhas da matriz de $(2.3)$ correspondentes a $\mathcal{C}^{*}$ formam uma matriz totalmente unimodular. Pelo teorema 1.9, o sistema (2.2) é box-TDI. 
Vamos ver agora que um célebre resultado de Lucchesi E Younger [1978] pode ser facilmente obtido do arcabouço de Edmonds e Giles.

\section{Corolário 2.3.1 (Teorema de Lucchesi-Younger)}

Num grafo orientado, o tamanho mínimo de uma junção é igual ao número máximo de cortes orientados disjuntos.

Demonstração. Segue da proposição 1.8, do teorema 1.7 e do teorema 2.3 de Edmonds-Giles aplicados ao programa linear $\max \{c x: x$ é um fluxo submodular e $\ell \leq x \leq u\}$, onde $c:=\mathbf{1}$, $\mathcal{C}:=\{S: \varnothing \neq S \subsetneq V$ e $\overleftarrow{\delta}(S)=\varnothing\}, f:=-\mathbf{1}, \ell:=-\infty$ e $u:=\mathbf{0}$

É similarmente fácil mostrar que o teorema 2.3 de Edmonds-Giles implica o teorema de intersecção de polimatróides de EDMONDS [1970], uma generalização do famoso teorema de intersecção de matróides de Edmonds, que derivamos a seguir:

Corolário 2.3.2 (Teorema de intersecção de matróides de Edmonds)

Sejam $M_{1}=\left(E, \mathcal{I}_{1}\right)$ e $M_{2}=\left(E, \mathcal{I}_{2}\right)$ matróides. Seja $r_{i}$ a função posto do matróide $M_{i}$ para $i=1,2$. Então o tamanho máximo de um conjunto em $\mathcal{I}_{1} \cap \mathcal{I}_{2}$ é

$$
\min _{F \subseteq E}\left\{r_{1}(F)+r_{2}(E \backslash F)\right\}
$$

Demonstração. Sejam $E^{\prime}$ e $E^{\prime \prime}$ duas cópias disjuntas do conjunto $E$. Tome $V:=E^{\prime} \cup E^{\prime \prime}$ e $A:=\left\{\left(e^{\prime \prime}, e^{\prime}\right): e \in E\right\}$. Vamos aplicar o teorema 2.3 de Edmonds-Giles sobre o grafo orientado $D:=(V, A)$.

Tome $\mathcal{C}:=\left\{F^{\prime}: F \subseteq E\right\} \cup\left\{E^{\prime} \cup F^{\prime \prime}: F \subseteq E\right\}$, onde $F^{\prime}$ e $F^{\prime \prime}$ denotam o conjunto de cópias dos elementos de $F$ em $E^{\prime}$ e $E^{\prime \prime}$, respectivamente. Defina $f: \mathcal{C} \rightarrow \mathbb{Z}_{\geq 0}$ como

$$
\begin{aligned}
f\left(F^{\prime}\right) & :=r_{1}(F), \quad \text { para todo } F \subsetneq E, \\
f\left(V \backslash F^{\prime \prime}\right) & :=r_{2}(F), \quad \text { para todo } F \subsetneq E, \\
f\left(E^{\prime}\right) & :=\min \left\{r_{1}(E), r_{2}(E)\right\} .
\end{aligned}
$$

Agora basta aplicar a proposição 1.8, o teorema 1.7 e o teorema 2.3 de Edmonds-Giles ao programa linear $\max \{c x: x$ é um fluxo submodular e $\ell \leq x \leq u\}$, onde $c:=\mathbf{1}, \ell:=\mathbf{0}$ e $u:=\infty$.

Neste texto, não trataremos de algoritmos para encontrar um fluxo submodular de peso máximo. Porém, no capítulo 3, apresentaremos o algoritmo de FrAnK [1981a] para o teorema de Lucchesi-Younger, que é uma especialização do algoritmo de FrAnK [1982] para um caso particular de fluxos submodulares. Esperamos assim que o leitor vislumbre o funcionamento desses algoritmos. As notas no final do capítulo mencionam alguns aspectos algorítmicos de fluxos submodulares e mais aplicações. 


\subsection{Uma variante}

Além dos fluxos submodulares de Edmonds e Giles, vários arcabouços semelhantes foram propostos para generalizar relações min-max e outros resultados. Destacamos os seguintes: cut-set polyhedra de JoHnson [1975], lattice polyhedra de HofFMan [1976, 1978] e Hoffman E Schwartz [1978], kernel systems de Frank [1979b], distributive lattices de Gröflin E HofFman [1982], polymatroidal network flows de HASsin [1982] e LAWLER E MARTEL [1982b, 1982a], e generalized polymatroids de Frank [1984b] e Frank E TARdos [1988].

SCHRIJVER [1984a] desenvolveu um arcabouço bastante geral, a partir do qual todos os modelos mencionados no parágrafo anterior podem ser obtidos. As relações entre eles podem ser vistas na excelente resenha de ScHRIJVER [1984b].

Nesta seção, modificamos levemente a demonstração do teorema 2.3 de Edmonds-Giles para obter uma variante devida a SCHRIJVER [1982]. A partir desse novo modelo, derivamos mais relações min-max.

Começamos adaptando o corolário 2.2.1:

\section{Lema $2.4 \alpha$}

Seja $D=(V, A)$ um grafo orientado e $\mathcal{C}$ uma coleção de subconjuntos de $V$. Seja $N$ a matriz $\mathcal{C} \times A$ definida como

$$
N[S, a]:= \begin{cases}1, & \text { se } a \in \vec{\delta}(S), \\ 0, & \text { caso contrário, }\end{cases}
$$

para cada $S \in \mathcal{C}$ e $a \in A$. Suponha que $\mathcal{C}$ satisfaz a seguinte condição:

$$
\text { se } X, Y, Z \in \mathcal{C} \text { e } X \subseteq V \backslash Y \subseteq Z \text {, então } \vec{\delta}(X) \cap \vec{\delta}(Z)=\varnothing
$$

Se $\mathcal{C}$ é livre de cruzamentos, então a matriz $N$ é totalmente unimodular.

Demonstração. Pelo teorema 2.2, a família $\mathcal{C}$ admite uma representação arbórea $(T, \pi)$. Seja $a=u v \in A$ e $P$ o pseudocaminho que liga $\pi(u)$ a $\pi(v)$ em $T$. Afirmamos que os arcos diretos de $P$ são consecutivos, isto é, formam um caminho $P^{\prime}$. Caso contrário, existiriam $\operatorname{arcos} b, c, d \in A_{T}$ que aparecem nesta ordem em $P$ e tais que $b$ e $d$ são arcos diretos de $P$ e $c$ é um arco reverso de $P$. Mas então a condição (2.7) é violada pelos conjuntos $X:=S_{d}$, $Y:=S_{c}$ e $Z:=S_{b}$, contradizendo nossa hipótese. Se $P^{\prime}$ vai de $u^{\prime}$ a $v^{\prime}$, defina $a^{\prime}:=u^{\prime} v^{\prime}$.

Tome o grafo orientado $D^{\prime}:=\left(V_{T}, A^{\prime}\right)$, onde $A^{\prime}:=\left\{a^{\prime}: a \in A\right\}$. Não é difícil ver que a matriz de rede gerada por $T$ e $D^{\prime}$ é igual a $N$, identificando cada arco $a \in A_{T}$ com o conjunto $S_{a} \in \mathcal{C}$ e cada arco $a^{\prime} \in A^{\prime}$ com o arco $a \in A$. Logo, pelo teorema 2.1 , a matriz $N$ é totalmente unimodular.

Seja $D=(V, A)$ um grafo orientado e $\mathcal{C}$ uma coleção de subconjuntos de $V$ fechada sob cruzamentos. Uma função $g: \mathcal{C} \rightarrow \mathbb{R}$ é dita supermodular em cruzamentos se a função $-g$ é submodular em cruzamentos. 


\section{Teorema 2.4 (Variante de Schrijver)}

Seja $D=(V, A)$ um grafo orientado. Seja $\mathcal{C}$ uma coleção de subconjuntos de $V$ fechada sob cruzamentos e $g: \mathcal{C} \rightarrow \mathbb{R}$ uma função supermodular em cruzamentos. Se a coleção $\mathcal{C}$ satisfaz a condição (2.7), então o sistema

$$
\begin{array}{ll}
x_{a} \geq 0 & \text { para todo } a \in A, \\
x(\vec{\delta}(S)) \geq g(S) & \text { para todo } S \in \mathcal{C},
\end{array}
$$

é box-TDI.

Demonstração. A prova é quase idêntica à do teorema 2.3 de Edmonds-Giles. Dentre todas as soluções ótimas $y$ do programa linear dual a $\min \{c x: x$ satisfaz (2.8) $\}$, escolha como $y^{*}$ uma solução que minimiza a função linear $\phi(y)$ definida em (2.5).

Para ver que $\mathcal{C}^{*}:=\left\{S \in \mathcal{C}: y_{S}^{*}>0\right\}$ é livre de cruzamentos, suponha que $U, W \in \mathcal{C}^{*}$ se cruzam. Defina $y^{\prime} \in \mathbb{R}_{\geq 0}^{\mathcal{C}}$ como em (2.6). Como

$$
\chi^{\vec{\delta}(U \cap W)}+\chi^{\vec{\delta}(U \cup W)} \leq \chi^{\vec{\delta}(U)}+\chi^{\vec{\delta}(W)}
$$

e $g$ é supermodular em cruzamentos, então $y^{\prime}$ também é uma solução ótima do dual. Porém, pelo lema $2.3 \alpha$ do descruzamento, temos $\phi\left(y^{\prime}\right)<\phi\left(y^{*}\right)$, contradizendo a escolha de $y^{*}$.

Como a coleção $\mathcal{C}^{*}$ é livre de cruzamentos, o lema $2.4 \alpha$ nos garante que as linhas da matriz de $(2.8)$ correspondentes a $\mathcal{C}^{*}$ formam uma matriz totalmente unimodular. Segue do teorema 1.9 que o sistema (2.8) é box-TDI.

Derivamos agora uma série de relações min-max. Observe que, pela proposição 1.8 e pelos teoremas 1.7 e 2.4 ,

$$
\min \left\{c x: x \in \mathbb{Z}_{\geq 0}^{A}, x \text { satisfaz }(2.8)\right\}=\max \left\{y g: y \in \mathbb{Z}_{\geq 0}^{\mathcal{C}}, \sum_{S \in \mathcal{C}} y_{S} \chi^{\vec{\delta}(S)} \leq c\right\}
$$

sempre que $g$ e $c$ são vetores integrais e algum desses programas lineares admite alguma solução ótima. Nos corolários a seguir, sempre usaremos $g:=\mathbf{1}$ e definiremos apenas os parâmetros $D, c$ e $\mathcal{C}$ satisfazendo as hipóteses do teorema 2.4 .

Seja $G=(V, E)$ um grafo. Dizemos que $F \subseteq E$ é uma cobertura por arestas se cada vértice de $G$ é ponta de alguma aresta de $F$. Dizemos que $S \subseteq V$ é estável se o subgrafo induzido $G[S]$ não tem arestas. RADO [1933] obteve a seguinte relação min-max, que GALLAI [1958a, 1958b] atribui a König em 1932:

\section{Corolário 2.4.1 (Teorema de cobertura por arestas de König-Rado)}

Num grafo bipartido sem vértices isolados, o tamanho mínimo de uma cobertura por arestas é igual ao tamanho máximo de um conjunto estável.

Demonstração. Seja $G$ um grafo $(U, W)$-bipartido. Oriente todas as arestas de $U$ para $W$, obtendo o grafo orientado $D$. Agora o resultado segue da equação (2.9) tomando $c:=1$ e $\mathcal{C}:=\left\{V_{G} \backslash\{u\}: u \in U\right\} \cup\{\{w\}: w \in W\}$. 
Seja $D=(V, A)$ um grafo orientado e $r \in V$. Dizemos que $B \subseteq A$ é um $r$-corte ou um corte enraizado em $r$ se $B=\vec{\delta}(S)$ para algum subconjunto não-vazio $S \subseteq V \backslash\{r\}$. É óbvio que um subconjunto de arcos contém uma $r$-arborescência se, e somente se, intersecta todos os $r$-cortes. A seguinte relação min-max foi provada por FULKERSON [1974] e já aparecia implícita nos artigos de EDMONDS [1967] e Bock [1971].

Corolário 2.4.2 (Teorema da arborescência ótima de Fulkerson)

Seja $D=(V, A)$ um grafo orientado, $\ell: A \rightarrow \mathbb{Z}_{\geq 0}$ uma função comprimento e $r \in V$. Então o comprimento mínimo de uma $r$-arborescência é igual ao tamanho máximo de uma família $\ell$-disjunta de $r$-cortes.

Demonstração. Imediato de (2.9), tomando $c:=\ell$ e $\mathcal{C}:=\{S: \varnothing \neq S \subseteq V \backslash\{r\}\}$.

Seja $D=(V, A)$ um grafo orientado e $\{R, S\}$ uma partição de $V$. Dizemos que $B \subseteq A$ é uma $(R, S)$-bi-ramificação se, no grafo orientado $(V, B)$, cada vértice de $S$ é acessível a partir de algum vértice de $R$ e cada vértice de $R$ acessa algum vértice de $S$. Observe que $B \subseteq A$ é uma $(R, S)$-bi-ramificação se, e somente se, o grafo orientado $(V, B) / R$ contém uma $R$-arborescência e o grafo orientado $\left(V, B^{-1}\right) / S$ contém uma $S$-arborescência, onde $R$ e $S$ denotam os vértices contraídos.

Dizemos que $B \subseteq A$ é um $(R, S)$-bicorte se $B=\vec{\delta}(U)$ para algum subconjunto não-vazio $U \subsetneq V$ tal que $U \subseteq S$ ou $U \supseteq S$. É evidente que um subconjunto de arcos contém uma $(R, S)$-bi-ramificação se, e somente se, intersecta todos os $(R, S)$-bicortes. O resultado a seguir foi obtido por SCHRIJVER [1982]:

\section{Corolário 2.4.3 (Teorema da bi-ramificação ótima de Schrijver)}

Seja $D=(V, A)$ um grafo orientado, $\ell: A \rightarrow \mathbb{Z}_{\geq 0}$ uma função comprimento e $\{R, S\}$ uma partição de $V$. Então o comprimento mínimo de uma $(R, S)$-bi-ramificação é igual ao tamanho máximo de uma família $\ell$-disjunta de $(R, S)$-bicortes.

Demonstração. Imediato de (2.9), $\operatorname{com} c:=\ell$ e $\mathcal{C}:=\{U: \varnothing \neq U \subseteq S$ ou $S \subseteq U \subsetneq V\}$.

O teorema 2.3.1 de Lucchesi-Younger e o teorema 2.3.2 de intersecção de matróides de Edmonds também podem ser facilmente derivados da variante de Schrijver. Notamos ainda que, para derivar versões capacitadas dos teoremas anteriores, basta aplicar a equação (2.9) com uma função custo $c: A \rightarrow \mathbb{Z}_{\geq 0}$ arbitrária.

No capítulo 3, veremos um outro arcabouço para relações min-max: os conectores fortes de SchriJver [1982]. O teorema do conector forte ótimo de Schrijver também pode ser derivado da variante de Schrijver. Omitimos a prova, já que veremos depois uma redução algorítmica desse modelo para o teorema de Lucchesi-Younger. 


\section{Resultados relacionados}

As matrizes de rede são, na verdade, os blocos básicos de construção de todas as matrizes totalmente unimodulares: segundo um profundo teorema estrutural de SEYmour [1980], toda matriz totalmente unimodular pode ser construída a partir de matrizes de rede e de duas matrizes $5 \times 5$ através de certas operações de "colagem". Mais detalhes sobre a teoria relacionada a unimodularidade total podem ser encontrados no livro de programação linear e inteira de SchriJver [1986, capítulos 19-21] e no livro de decomposição de matróides de Truemper [1992, capítulos 9-12].

Na seção 2.3, usamos fluxos submodulares para provar duas relações min-max: o teorema de Lucchesi-Younger e o teorema de intersecção de matróides de Edmonds. Porém, podemos derivar também resultados puramente combinatórios a partir desse arcabouço: FrANK [1982] mostrou que uma versão fraca do teorema de orientação de NASH-WILLIAMs [1960] segue do teorema 2.3 de Edmonds-Giles. Essa versão fraca afirma que um grafo admite uma orientação $k$-arco-conexa se, e somente se, é $2 k$-aresta-conexo. Veja também Frank [1980].

FRANK E TARDOS [1989] mostraram ainda que o teorema 2.3 de Edmonds-Giles pode ser usado para resolver o seguinte problema de aumento de conexidade enraizada. Dados grafos orientados $\left(V, A_{0}\right)$ e $(V, A)$, uma função custo $c: A \rightarrow \mathbb{R}_{\geq 0}$, um vértice $r \in V$ e um inteiro $k \in \mathbb{Z}_{\geq 0}$, encontre um subconjunto de $\operatorname{arcos} A^{\prime} \subseteq A$ de custo mínimo tal que, para cada $v \in V$, o grafo orientado $\left(V, A_{0} \cup A^{\prime}\right)$ tenha $k$ caminhos de $r$ a $v$ internamente disjuntos. Veja também Frank [1999b].

A seguir, listamos algumas referências sobre aspectos algorítmicos de fluxos submodulares.

O primeiro algoritmo polinomial para fluxos submodulares foi obtido por GrötscheL, LovÁsz E SchriJver [1981] e é baseado no método dos elipsóides. O primeiro algoritmo combinatório polinomial foi desenvolvido por Cunningham E Frank [1985], utilizando a regra de caminhos aumentadores em ordem lexicográfica de SchÖNSLEBEN [1980] e LAWLER E MARTEL [1982a], a técnica de scaling de Edmonds E KARP [1972] e RöcK [1980], e idéias e resultados de FRANK [1982, 1984a].

FRANK E TARDOS [1987] obtiveram o primeiro algoritmo fortemente polinomial para o problema do fluxo submodular de peso máximo, baseando-se no método de aproximação diofantina simultânea de Lenstra, Lenstra e Lovász [1982]. Fujishige, Zimmerman E RöCK [1989] desenvolveram um algoritmo fortemente polinomial que é uma generalização mais direta do algoritmo de TARDOS [1985] para o problema da circulação de custo mínimo, o primeiro algoritmo fortemente polinomial para este problema. Esse algoritmo de Fujishige, Zimmerman e Röck usa como sub-rotina o algoritmo de Cunningham e Frank e o método de tree projection de FuJishige [1986].

Todos esses algoritmos supõem a existência de um procedimento capaz de minimizar uma função submodular arbitrária. Um tal procedimento já era conhecido para diversos casos especiais importantes. Para o caso geral, o primeiro algoritmo fortemente polinomial foi construído por Grötschel, Lovász e Schrijver [1981, 1988] e é baseado no método dos elipsóides. O desenvolvimento de um algoritmo combinatório fortemente polinomial para minimizar uma função submodular arbitrária manteve-se um problema em aberto por quase 20 anos, tendo sido resolvido em 1999 independentemente por SchriJver [2000] e IWATA, 
Fleischer e Fujishige [2001]. Os dois algoritmos foram inspirados em trabalhos anteriores de Cunningham [1984, 1985].

Para resultados mais recentes nessa linha de pesquisa, recomendamos o artigo de IWATA, McCormick e Shigeno [2005] e a monografia de Fujishige [2005].

LovÁsz [1976] forneceu uma prova combinatória do teorema 2.3.1 de Lucchesi-Younger, baseada em submodularidade.

A técnica do descruzamento também foi usada para provar uma relação min-max bastante geral de FRANK E JORDÁN [1995] para cobertura bisupermodular, que inclui alguns problemas de aumento de conexidade, o teorema de EDMONDS [1965b] sobre partição do conjunto-base de um matróide em bases, e uma extensão de um teorema de LuBIW [1991], que é uma versão capacitada de uma relação min-max de GYŐRI [1984] sobre intervalos. Veja também FRANK [1999a]. 


\section{Capítulo 3}

\section{O algoritmo de Frank}

O primeiro algoritmo fortemente polinomial para encontrar uma junção de custo mínimo foi desenvolvido por Frank. Esse algoritmo resolve simultaneamente os dois problemas de otimização de uma versão capacitada do teorema de Lucchesi-Younger: o problema da junção de custo mínimo, e o seu dual, que envolve o empacotamento de cortes orientados. Neste capítulo, apresentamos o algoritmo de Frank e o arcabouço de conectores fortes de custo mínimo de Schrijver, que fornece uma redução algorítmica de quase todas as relações min-max derivadas no capítulo 2 para o teorema de Lucchesi-Younger. Teremos assim um algoritmo fortemente polinomial para cada uma dessas relações.

Como já mencionamos na seção 2.3, o algoritmo de Frank para a versão capacitada do teorema de Lucchesi-Younger é, na verdade, uma especialização de um algoritmo mais geral para fluxos submodulares. Porém, as principais características desse algoritmo mais geral estão presentes nessa especialização. Uma boa compreensão do algoritmo de Frank para junções de custo mínimo facilita muito o estudo do algoritmo para fluxos submodulares.

O algoritmo é primal-dual e mantém, a cada iteração, uma junção e uma certa função, chamada potencial. A cada iteração, alteramos um desses objetos para que eles se aproximem de certas condições de otimalidade. A principal operação do algoritmo é a modificação da junção atual ao longo de um certo circuito num grafo auxiliar. No fim do algoritmo, quando as condições de otimalidade estão satisfeitas, usamos a função potencial para construir um empacotamento de cortes orientados que certifica que a junção obtida tem custo mínimo.

O restante do capítulo está organizado da seguinte forma: na seção 3.1, estudamos diversas propriedades de núcleos, objetos centrais no desenvolvimento do algoritmo; na seção 3.2, enunciamos as condições de otimalidade do algoritmo; na seção 3.3, mostramos como uma junção pode ser modificada ao longo de um circuito num certo grafo auxiliar; na seção 3.4, descrevemos a iteração primal-dual do algoritmo, encaixando as peças desenvolvidas nas seções anteriores; na seção 3.5, finalizamos a descrição do algoritmo ao mostrarmos como construir o grafo auxiliar de forma eficiente; concluímos o capítulo com a seção 3.6, na qual descrevemos o arcabouço de conectores fortes de custo mínimo de Schrijver. 


\section{$3.1 \quad$ Núcleos}

Seja $D=(V, A)$ um grafo orientado fracamente conexo. Um subconjunto não-vazio $N \subseteq V$ é um núcleo se $\overleftarrow{\delta}(N)=\varnothing$, isto é, se $N=V$ ou $\vec{\delta}(N)$ é um corte orientado. É fácil ver que

$$
\vec{d}(N \cap M)+\vec{d}(N \cup M)=\vec{d}(N)+\vec{d}(M)
$$

para quaisquer núcleos $N$ e $M$.

Para cada $S \subseteq V$, denote por $\kappa(S)$ o número de componentes fracos de $D-S$, sendo $\kappa(V)=0$ por convenção.

Para quaisquer núcleos $N$ e $M$, temos $\kappa(N)+\kappa(M) \leq \kappa(N \cap M)+\kappa(N \cup M)$.

Demonstração. Seja $G$ o grafo subjacente a $D$. Abrevie $\bar{E}[S]:=E_{G}[V \backslash S]$ para todo $S \subseteq V$. Vamos usar a função submodular $r: \mathcal{P}\left(E_{G}\right) \rightarrow \mathbb{R}$ definida no corolário 1.12.1. Observe que $r(\bar{E}[S])=|V|-|S|-\kappa(S)$ para todo $S \subseteq V$.

É fácil ver que $\bar{E}[N \cup M]=\bar{E}[N] \cap \bar{E}[M]$. Como $N$ e $M$ são núcleos, então não há arestas com uma ponta em $N \backslash M$ e outra em $M \backslash N$. Usando esse fato, é fácil provar que $\bar{E}[N \cap M]=\bar{E}[N] \cup \bar{E}[M]$. Pela submodularidade da função $r$, temos

$$
\begin{aligned}
\kappa(N)+\kappa(M) & =|V|-|N|-r(\bar{E}[N])+|V|-|M|-r(\bar{E}[M]) \\
& \leq|V|-|N \cap M|-r(\bar{E}[N] \cup \bar{E}[M])+|V|-|N \cup M|-r(\bar{E}[N] \cap \bar{E}[M]) \\
& =|V|-|N \cap M|-r(\bar{E}[N \cap M])+|V|-|N \cup M|-r(\bar{E}[N \cup M]) \\
& =\kappa(N \cap M)+\kappa(N \cup M),
\end{aligned}
$$

como queríamos.

Se $N \neq V$ é um núcleo, então o corte orientado $\vec{\delta}(N)$ é a união de $\kappa(N)$ cortes orientados disjuntos. Para ver isso, defina $\mathcal{L}(N):=\{V \backslash K: K$ é um componente fraco de $D-N\}$ e note que $\vec{\delta}(N)=\bigcup\{\vec{\delta}(M): M \in \mathcal{L}(N)\}$. Concluímos assim que,

$$
\text { se } N \text { é um núcleo e } J \text { é uma junção, então } \vec{d}_{J}(N) \geq \kappa(N) \text {. }
$$

Seja $J$ uma junção. Dizemos que o núcleo $N$ é justo (com relação a $J$ ) se $\vec{d}_{J}(N)=\kappa(N)$. Se, além disso, $\kappa(N)=1$, dizemos que $N$ é perigoso (com relação a $J$ ). Dada uma família $\mathcal{N}$ de núcleos, adote a abreviatura $\vec{\delta}(\mathcal{N}):=\{\vec{\delta}(N): N \in \mathcal{N}\}$. Concluímos da discussão anterior que,

se $N$ é um núcleo justo, então $\mathcal{L}(N)$ consiste de núcleos perigosos e $\vec{\delta}(\mathcal{L}(N))$ é uma partição de $\vec{\delta}(N)$.

O seguinte argumento é bastante comum em provas envolvendo submodularidade:

Sejam $N$ e $M$ núcleos justos com $N \cap M \neq \varnothing$. Então $N \cap M$ e $N \cup M$ também são núcleos justos. 
Demonstração. É evidente que $N \cap M$ e $N \cup M$ são núcleos. Pela equação (3.1) e pelas afirmações (3.2) e (3.3),

$$
\begin{aligned}
\kappa(N)+\kappa(M) & =\vec{d}_{J}(N)+\vec{d}_{J}(M)=\vec{d}_{J}(N \cap M)+\vec{d}_{J}(N \cup M) \\
& \geq \kappa(N \cap M)+\kappa(N \cup M) \geq \kappa(N)+\kappa(M),
\end{aligned}
$$

de modo que todas as inequações acima valem com igualdade. Logo, $\vec{d}_{J}(N \cap M)=\kappa(N \cap M)$ e $\vec{d}_{J}(N \cup M)=\kappa(N \cup M)$.

Aplicando a afirmação (3.5) repetidas vezes, obtemos que,

se uma coleção de núcleos justos forma um hipergrafo conexo, então sua união é um núcleo justo.

Seja $v \in V$. Denote por $N_{\min }(v)$ a intersecção de todos os núcleos justos que contêm $v$. Observe que $N_{\min }(v)$ está bem definido, já que $V$ é um núcleo justo. Note ainda que $N_{\min }(v)$ depende da junção $J$ em questão. Segue de (3.5) que

$N_{\min }(v)$ é o menor núcleo justo que contém $v$, e $w \in N_{\min }(v)$ sempre que $v w \in A$.

Um núcleo $N$ é fechado (com relação a $J$ ) se $N_{\min }(v) \subseteq N$ para todo $v \in N$. Por exemplo, $V$ é um núcleo fechado. Então

um núcleo é fechado se, e somente se, é uma união de núcleos justos disjuntos.

Demonstração. É evidente que toda união de núcleos justos disjuntos é um núcleo fechado. Seja $N$ um núcleo fechado. Considere o hipergrafo $\mathcal{H}$ sobre $N \operatorname{com} \mathcal{E}_{\mathcal{H}}:=\left\{N_{\min }(v): v \in N\right\}$. Pela afirmação (3.6), os componentes de $\mathcal{H}$ são núcleos justos, e sua união é $N$.

A partição $\mathcal{N}$ de $N$ obtida nesta última prova é chamada de partição justa de $N$. Abreviamos $\mathcal{K}(N):=\bigcup_{M \in \mathcal{N}} \mathcal{L}(M)$. É claro que $\{\vec{\delta}(M): M \in \mathcal{N}\}$ é uma partição de $\vec{\delta}(N)$. Logo, pela afirmação (3.4), temos que,

se $N$ é um núcleo fechado, então $\mathcal{K}(N)$ consiste de núcleos perigosos e $\vec{\delta}(\mathcal{K}(N))$ é uma partição de $\vec{\delta}(N)$.

Vale também o seguinte:

Sejam $J$ e $J^{\prime}$ junções. Se $N$ é um núcleo fechado com relação a $J$ e $\vec{d}_{J^{\prime}}(N)=\vec{d}_{J}(N)$, então $N$ é fechado com relação a $J^{\prime}$.

Demonstração. Seja $\mathcal{N}$ a partição justa de $N$ com relação a $J$. Então

$$
\vec{d}_{J}(N)=\sum_{M \in \mathcal{N}} \vec{d}_{J}(M)=\sum_{M \in \mathcal{N}} \kappa(M) \leq \sum_{M \in \mathcal{N}} \vec{d}_{J^{\prime}}(M)=\vec{d}_{J^{\prime}}(N)=\vec{d}_{J}(N),
$$

de modo que cada $M \in \mathcal{N}$ é justo com relação a $J^{\prime}$. Agora o resultado segue de (3.8). 


\subsection{Condições de otimalidade e potenciais}

Seja $D=(V, A)$ um grafo orientado fracamente conexo e $c: A \rightarrow \mathbb{Z}_{\geq 0}$ uma função custo. Seja $J$ uma junção e $\mathcal{N}$ uma família de núcleos tal que cada arco $a \in A$ entra em no máximo $c(a)$ dos núcleos de $\mathcal{N}$. Uma simples contagem dupla mostra que

$$
c(J)=\sum_{a \in J} c(a) \geq \sum_{a \in J}|\{N \in \mathcal{N}: a \in \vec{\delta}(N)\}|=\sum_{N \in \mathcal{N}} \vec{d}_{J}(N) \geq \sum_{N \in \mathcal{N}} 1=|\mathcal{N}| .
$$

Em particular, o custo mínimo de uma junção é pelo menos o tamanho máximo de uma tal família de núcleos.

Queremos mostrar que sempre podemos encontrar uma junção e uma família de núcleos satisfazendo (3.11) com igualdade. Para tanto, basta exigirmos que todas as relações de (3.11) valham com igualdade, isto é, basta encontrarmos uma junção $J$ e uma família $\mathcal{N}$ de núcleos tais que

(i) todo arco $a \in J$ entra em exatamente $c(a)$ núcleos de $\mathcal{N}$,

(ii) todo arco $a \in A \backslash J$ entra em no máximo $c(a)$ núcleos de $\mathcal{N}$,

(iii) $\vec{d}_{J}(N)=1$ para todo $N \in \mathcal{N}$.

Na verdade, podemos enfraquecer a condição (3.12)(i) para a seguinte exigência:

$\left(\mathrm{i}^{\prime}\right)$ todo arco $a \in J$ entra em pelo menos $c(a)$ núcleos de $\mathcal{N}$.

De fato, suponha que $J$ e $\mathcal{N}$ satisfazem as condições (3.12)(i'), (3.12)(ii) e (3.12)(iii). Para cada arco $a \in J$ que entra em $k>c(a)$ núcleos de $\mathcal{N}$, remova $k-c(a)$ desses núcleos de $\mathcal{N}$. Após realizarmos esse ajuste para cada $a \in J$, obteremos uma família $\mathcal{N}^{\prime}$ de núcleos que, com $J$, satisfaz (3.12)(i), (3.12)(ii) e (3.12)(iii).

$\mathrm{O}$ algoritmo de Frank encontra uma junção $J$ e uma família $\mathcal{N}$ de núcleos satisfazendo as condições de otimalidade $(3.12)\left(\mathrm{i}^{\prime}\right),(3.12)(\mathrm{ii})$ e (3.12)(iii). O algoritmo é primal-dual. Nesse tipo de algoritmo, seria usual trabalharmos com um vetor $y$ indexado pelos núcleos de $D$. Note que esse vetor pode ter dimensão exponencial no tamanho da codificação de $D$. Porém, Frank introduziu uma idéia que nos permite trabalhar apenas com um vetor indexado por $V$, que chamamos de potencial. No fim do algoritmo, esse potencial nos permitirá construir uma família $\mathcal{N}$ de núcleos que tem tamanho máximo.

Definimos um potencial como uma função $p: V \rightarrow \mathbb{Z}$. Para cada arco $u v \in A$, defina o custo reduzido de $u v$ (com relação a $p$ ) como $c_{p}(u v):=c(u v)-p(v)+p(u)$.

Seja $J$ uma junção e $p$ um potencial satisfazendo as seguintes condições:

(i) se $a \in J$, então $c_{p}(a) \leq 0$,

(ii) se $a \in A \backslash J$, então $c_{p}(a) \geq 0$,

(iii) se $v \in N_{\min }(u)$, então $p(v) \geq p(u)$.

No restante desta seção, vamos mostrar que, a partir de $p$, podemos construir uma família $\mathcal{N}$ de núcleos que, com $J$, satisfaz as condições de otimalidade (3.12)(i'), (3.12)(ii) e (3.12)(iii). 
Vamos denotar por $p_{0}, \ldots, p_{k}$ os valores distintos que $p$ assume, com $p_{0}<\cdots<p_{k}$. Para $i=1, \ldots, k$, defina $N_{i}:=\left\{v: p(v) \geq p_{i}\right\}$. É claro que $\varnothing \neq N_{i} \subsetneq V$ para cada $i$, contanto que $c(J) \neq 0$. Ademais,

a condição (3.13)(iii) é equivalente ao fato de que cada $N_{i}$ é um núcleo fechado.

Demonstração. Primeiro vamos demonstrar que, se a condição (3.13)(iii) é satisfeita, então cada $N_{i}$ é um núcleo fechado. Para ver que $N_{i}$ é um núcleo, seja $u v \in A$ com $u \in N_{i}$. Por (3.7) e (3.13)(iii), temos que $v \in N_{i}$, de modo que $\overleftarrow{\delta}\left(N_{i}\right)=\varnothing$. É imediato de (3.13)(iii) que, para $i=1, \ldots, k$, vale que $N_{\min }(u) \subseteq N_{i}$ sempre que $u \in N_{i}$.

Resta provarmos que, se cada $N_{i}$ é um núcleo fechado, então a condição (3.13)(iii) é satisfeita. Sejam $u, v \in V \operatorname{com} v \in N_{\min }(u)$. Se $p(u)=p_{0}$, nada temos a demonstrar. Senão, como $N_{p(u)}$ é fechado, temos $v \in N_{p(u)}$, de modo que $p(v) \geq p(u)$, como queríamos.

Estamos prontos para construir nossa família $\mathcal{N}$ de núcleos. Na verdade, o que vamos construir é um vetor integral $y$ indexado pelos núcleos de $D$ de forma que, para cada núcleo $N$, o valor $y_{N}$ denota quantas cópias de $N$ aparecem em $\mathcal{N}$. Para cada núcleo $N \in \bigcup_{i=1}^{k} \mathcal{K}\left(N_{i}\right)$, tome $y_{N}:=\sum\left\{p_{i}-p_{i-1}: N \in \mathcal{K}\left(N_{i}\right)\right\}$. Se um núcleo $M$ não aparece em $\bigcup_{i=1}^{k} \mathcal{K}\left(N_{i}\right)$, tome $y_{M}:=0$. Note que cada arco $u v \in A$ entra em exatamente $p(v)-p(u)$ núcleos de $\mathcal{N}$.

Terminamos a seção provando que $J$ e $\mathcal{N}$ satisfazem as condições de otimalidade $(3.12)\left(\mathrm{i}^{\prime}\right)$, (3.12)(ii) e (3.12)(iii). A condição (3.13)(i) nos garante que $p(v)-p(u) \geq c(u v)$ para cada arco $u v \in J$, ou seja, $u v$ entra em pelo menos $c(u v)$ núcleos de $\mathcal{N}$, satisfazendo $(3.12)\left(\mathrm{i}^{\prime}\right)$. Analogamente, (3.13)(ii) garante que (3.12)(ii) é satisfeita. Finalmente, concluímos de (3.14) e (3.9) que todo núcleo de $\mathcal{N}$ é perigoso, isto é, a condição (3.12)(iii) é satisfeita.

Nas seções a seguir, desenvolveremos um algoritmo primal-dual que encontra uma junção $J$ e uma função potencial $p$ satisfazendo as condições de otimalidade (3.13).

\subsection{Um grafo auxiliar e circuitos axiais}

Nesta seção, descrevemos uma operação fundamental usada pelo algoritmo de Frank. Vamos definir um grafo orientado auxiliar e mostrar que, usando certos circuitos deste grafo, podemos alterar uma junção do grafo original.

Seja $D=(V, A)$ um grafo orientado fracamente conexo, $J$ uma junção e $p$ um potencial. Suponha que $J$ e $p$ satisfazem a condição (3.13)(iii).

O grafo auxiliar de $D$ relativo a $J$ e $p$ é o grafo orientado $D(J, p):=(V, A(J, p))$, onde $A(J, p):=A_{J} \cup A_{\bar{J}} \cup A_{a} \mathrm{e}$

$$
\begin{aligned}
& A_{J}:=J, \\
& A_{\bar{J}}:=(A \backslash J)^{-1}, \\
& A_{a}:=\left\{u v: v \in N_{\min }(u) \text { e } p(v)=p(u)\right\} .
\end{aligned}
$$

Note que, para obter $D(J, p)$ de $D$, basta inverter todos os arcos que não estão em $J$ e adicionar os arcos de $A_{a}$, chamados de arcos artificiais. Veremos na seção 3.5 como encontrar $N_{\min }(u)$ em tempo polinomial, o que nos permitirá construir o grafo auxiliar eficientemente. 
Seja $b=x y \in J$. Suponha que existe um caminho $P=\left\langle v_{0}, a_{1}, v_{1}, \ldots, a_{k}, v_{k}\right\rangle$ de $y$ para $x$ em $D(J, p)$, ou seja, $v_{0}=y$ e $v_{k}=x$, tal que,

para quaisquer $i, j$ tais que $0 \leq i, j \leq k$ e $j \geq i+2$, vale que $v_{i} v_{j} \notin A_{a}$.

Tome o circuito $C:=P+b$. Todo circuito que surge dessa maneira é chamado de axial. Denote $C_{J}:=C \cap A_{J}$ e $C_{\bar{J}}:=C \cap A_{\bar{J}}$.

Nosso objetivo nesta seção é mostrar que $J^{\prime}:=\left(J \backslash C_{J}\right) \cup C_{\bar{J}}^{-1}$ é uma junção de $D$. Poderemos então pensar em $J^{\prime}$ como a junção obtida girando $J$ em torno do circuito axial $C$.

Antes disso, vamos tentar entender melhor a escolha dos arcos do grafo auxiliar. Seja $N$ um núcleo perigoso com relação a $J$. Caminhando pelo circuito $C$, digamos que entramos em $N$ por um arco de $J$. Note que tal arco não estará mais em $J^{\prime}$. Como $C$ é um circuito, devemos eventualmente sair de $N$ se continuarmos caminhando por $C$. Mas nenhum arco artificial sai de um núcleo justo. Então a única maneira de sairmos de $N$ é por um arco de $A_{\bar{J}}$. Observe que o inverso desse arco fará parte de $J^{\prime}$, compensando a remoção do único arco de $J$ que entrava no núcleo $N$.

Assim, provamos informalmente que, para todo núcleo $N$ que é perigoso com relação a $J$, temos $\vec{d}_{J^{\prime}}(N) \geq 1$. Vamos ver a seguir que isso pode ser estendido a todos os núcleos de $D$ :

\section{Teorema 3.1}

Para qualquer circuito axial $C_{J}$, o conjunto de $\operatorname{arcos} J^{\prime}:=\left(J \backslash C_{J}\right) \cup C_{\bar{J}}^{-1}$ é uma junção de $D$.

Demonstração. Dado um núcleo $N$, denote por $\vec{d}_{a}(N)$ o número de $\operatorname{arcos}$ artificiais de $C$ que entram em $N$ e por $\overleftarrow{d}_{a}(N)$ o número de arcos artificiais de $C$ que saem de $N$.

Começamos mostrando que, para qualquer núcleo $N$,

$$
\vec{d}_{J^{\prime}}(N)=\vec{d}_{J}(N)+\vec{d}_{a}(N)-\overleftarrow{d}_{a}(N)
$$

Isso é óbvio quando $\vec{d}_{a}(N)=\overleftarrow{d}_{a}(N)=0$. Suponha que $\vec{d}_{a}(N)>0$. Seja $a_{i}$ um arco artificial de $C$ que entra em $N$. Seja $a_{j}$ o próximo arco de $C$, em sua ordem cíclica, que sai de $N$. Se $a_{j}$ é artificial, então $a_{i}$ e $a_{j}$ não contribuem com nada para a soma (3.16). Se $a_{j}$ não é artificial, então $a_{j} \in A_{\bar{J}}$ e $a_{j}^{-1} \in J^{\prime}$, de modo que $a_{j}$ contribui com 1 para cada lado da soma (3.16). Observe que ambos os casos são consistentes com o valor de $\vec{d}_{J^{\prime}}(N)$. Um raciocínio análogo se aplica a arcos artificiais de $C$ que saem de $N$. Completamos assim a prova de (3.16).

Defina a folga de um núcleo $N$ como $f(N):=\vec{d}_{J}(N)-\kappa(N)$. Observe que $f(N) \geq 0$, com igualdade se, e somente se, $N$ é justo com relação a $J$. Por (3.1) e (3.2),

$$
f(N)+f(M) \geq f(N \cap M)+f(N \cup M)
$$

para quaisquer núcleos $N$ e $M$.

Vamos mostrar que

$$
f(N) \geq \overleftarrow{d}_{a}(N)
$$

para todo núcleo $N$. Antes, precisamos de um fato auxiliar. 
Seja $N$ um núcleo com $\overleftarrow{d}_{a}(N)>0$. Seja $a_{i}=u w$ um arco artificial de $C$ que sai de $N$ e tal que $p(w)$ é o máximo possível; se houver mais de um arco desse tipo, escolha um tal arco com $i$ mínimo. Defina $N^{\prime}:=N \cup N_{\min }(u)$, onde $N_{\min }(u)$ é relativo a $J$. Então

$$
\overleftarrow{d}_{a}\left(N^{\prime}\right)=\overleftarrow{d}_{a}(N)-1
$$

De fato, como nenhum arco artificial sai de $N_{\min }(u)$ e uw não sai de $N^{\prime}$, é evidente que $\overleftarrow{d}_{a}\left(N^{\prime}\right) \leq \overleftarrow{d}_{a}(N)-1$. Para ver que vale a igualdade, seja st um outro arco artificial de $C$ que sai de $N$. Afirmamos que $t \notin N_{\min }(u)$. Pois suponha que $t \in N_{\min }(u)$. Como $J$ e $p$ satisfazem a condição (3.13)(iii) por hipótese, temos $p(t) \geq p(u)$ e, pela maximalidade de $p(w)=p(u)$, temos $p(t)=p(u)$. Mas então, pela minimalidade de $i$, o arco $u t \in A_{a}$ viola a hipótese (3.15). Assim, $t \notin N_{\min }(u)$, de modo que o arco st sai de $N^{\prime}=N \cup N_{\min }(u)$, completando a prova de (3.19).

Podemos agora provar a afirmação (3.18). A prova é por indução em $\overleftarrow{d}_{a}(N)$. Se $\overleftarrow{d}_{a}(N)=0$, a afirmação vale trivialmente. Suponha então que $\overleftarrow{d}_{a}(N)>0$. Defina o vértice $u$ e o núcleo $N^{\prime}$ como no parágrafo anterior. Por (3.17), temos

$$
f(N)=f(N)+f\left(N_{\min }(u)\right) \geq f\left(N \cap N_{\min }(u)\right)+f\left(N^{\prime}\right) \geq 1+f\left(N^{\prime}\right),
$$

onde usamos a relação $N \cap N_{\min }(u) \subsetneq N_{\min }(u)$ para concluirmos que $N \cap N_{\min }(u)$ não é justo, ou seja, $f\left(N \cap N_{\min }(u)\right) \geq 1$. Por (3.19), podemos aplicar a hipótese de indução a $N^{\prime}$ para obtermos $f(N) \geq f\left(N^{\prime}\right)+1 \geq \overleftarrow{d}_{a}\left(N^{\prime}\right)+1=\overleftarrow{d}_{a}(N)$, concluindo a prova de (3.18).

Finalmente podemos provar o teorema. Basta mostrarmos que $\vec{d}_{J^{\prime}}(N) \geq \kappa(N)$ para todo núcleo $N$. Usando (3.16) e (3.18), temos

$$
\vec{d}_{J^{\prime}}(N)=\vec{d}_{J}(N)+\vec{d}_{a}(N)-\overleftarrow{d}_{a}(N)=\kappa(N)+f(N)+\vec{d}_{a}(N)-\overleftarrow{d}_{a}(N) \geq \kappa(N)+\vec{d}_{a}(N),
$$

como queríamos.

\subsection{A iteração primal-dual}

Podemos finalmente descrever o algoritmo primal-dual de FrAnK [1981a], que é uma especialização do algoritmo de FRANK [1982] para um caso particular de fluxos submodulares.

O algoritmo mantém uma junção $J$ e um potencial $p$. A cada iteração, eles são alterados de modo a se aproximarem das condições de otimalidade dadas por (3.13). Mais precisamente, $J$ e $p$ sempre satisfazem as condições (3.13)(ii) e (3.13)(iii) e, a cada iteração, o número de arcos de $J$ que violam (3.13)(i) diminui.

Uma iteração típica começa com uma junção $J$ e um potencial $p$ satisfazendo as condições (3.13)(ii) e (3.13)(iii) e um arco $b \in J$ que viola (3.13)(i). Ao fim da iteração, teremos uma junção $J^{\prime}$ e um potencial $p^{\prime}$ que satisfazem as condições (3.13)(ii) e (3.13)(iii), tal que o arco $b$ não viola (3.13)(i) com relação a $J^{\prime}$ e $p^{\prime}$ e, se um arco $a$ viola (3.13)(i) com relação a $J^{\prime}$ e $p^{\prime}$, então $a$ viola (3.13)(i) com relação a $J$ e $p$.

Na primeira iteração, podemos tomar como $J$ qualquer conjunto de arcos $B \subseteq A$ tal que o grafo subjacente a $(V, B)$ é uma árvore. Note que podemos obviamente supor que o grafo orientado de entrada é fracamente conexo. Podemos escolher ainda o potencial $p:=\mathbf{0}$. Assim, após no máximo $|V|-1$ iterações, teremos obtido uma junção $J$ e um potencial $p$ satisfazendo as condições de otimalidade (3.13). 
Vamos descrever agora uma iteração típica. Seja $J$ uma junção e $p$ um potencial que satisfazem (3.13)(ii) e (3.13)(iii). Seja $b=x y \in J$ um arco que viola (3.13)(i). Defina o grafo residual de $D$ relativo a $J$ e $p$ como $D^{\prime}(J, p):=\left(V, A^{\prime}(J, p)\right)$, onde $A^{\prime}(J, p):=A_{J}^{\prime} \cup A_{\bar{J}}^{\prime} \cup A_{a}^{\prime}$ e

$$
\begin{aligned}
& A_{J}^{\prime}:=\left\{u v \in J: c_{p}(u v) \geq 0\right\}, \\
& A_{\bar{J}}^{\prime}:=\left\{v u \in(A \backslash J)^{-1}: c_{p}(u v) \leq 0\right\}, \\
& A_{a}^{\prime}:=\left\{u v: v \in N_{\min }(u) \text { e } p(v)=p(u)\right\} .
\end{aligned}
$$

Note que $D^{\prime}(J, p)$ é um subgrafo do grafo auxiliar $D(J, p)$ e que $A_{a}^{\prime}=A_{a}$.

Nosso objetivo é encontrar em $D^{\prime}(J, p)$ um caminho de $y$ a $x$. Seja $R$ o conjunto dos vértices de $V$ acessíveis a partir de $y$ em $D^{\prime}(J, p)$. Temos duas possibilidades.

CASO 1: não existe um caminho de $y$ a $x$ em $D^{\prime}(J, p)$, ou seja, $x \notin R$.

Defina

$$
\varepsilon:=\min \left\{\varepsilon_{b}, \varepsilon_{J}, \varepsilon_{\bar{J}}, \varepsilon_{a}\right\}
$$

onde

$$
\begin{aligned}
& \varepsilon_{b}:=c_{p}(x y), \\
& \varepsilon_{J}:=\min \left\{-c_{p}(u v): u v \in J \text { e } u v \text { sai de } R\right\}, \\
& \varepsilon_{\bar{J}}:=\min \left\{c_{p}(u v): v u \in(A \backslash J)^{-1} \text { e } v u \text { sai de } R\right\}, \\
& \varepsilon_{a}:=\min \left\{p(v)-p(u): v \in N_{\min }(u) \text { e } u v \text { sai de } R\right\} .
\end{aligned}
$$

Então

$$
\varepsilon>0
$$

Demonstração. Como o arco $b$ viola (3.13)(i) por hipótese, então $\varepsilon_{b}>0$. Se um arco $u v \in J$ sai de $R$, então $c_{p}(u v)<0$, pois caso contrário teríamos $u v \in A_{J}^{\prime}$, e portanto $v \in R$. Logo, $\varepsilon_{J}>0$. Se um arco $v u \in(A \backslash J)^{-1}$ sai de $R$, então $c_{p}(u v)>0$, pois caso contrário teríamos $v u \in A_{\bar{J}}^{\prime}$, e portanto $u \in R$. Logo, $\varepsilon_{\bar{J}}>0$. Finalmente, se $p(v)-p(u) \leq 0$ para certos $u, v \in V$ com $u \in R$ e $v \in N_{\min }(u) \backslash R$, então obtemos de (3.13)(iii) que $p(u)=p(v)$, de modo de teríamos $u v \in A_{a}^{\prime}$, e portanto $v \in R$. Logo, $\varepsilon_{a}>0$.

Construa um novo potencial $p^{\prime}$ tomando

$$
p^{\prime}(v):= \begin{cases}p(v)+\varepsilon, & \text { se } v \in R, \\ p(v), & \text { se } v \notin R .\end{cases}
$$

Conseqüentemente, para todo arco $u v \in A$, temos

$$
c_{p^{\prime}}(u v)= \begin{cases}c_{p}(u v)+\varepsilon, & \text { se } u v \text { sai de } R, \\ c_{p}(u v)-\varepsilon, & \text { se } u v \text { entra em } R, \\ c_{p}(u v), & \text { caso contrário. }\end{cases}
$$

Por construção,

as condições (3.13)(ii) e (3.13)(iii) continuam satisfeitas com relação a $p^{\prime}$. 
Demonstração. Seja $u v \in A \backslash J$. Como a condição (3.13)(ii) é satisfeita com relação a $J$ e $p$, então $c_{p}(u v) \geq 0$. Se $c_{p^{\prime}}(u v)<0$, concluímos de (3.23) que uv entra em $R$. Mas então temos $\varepsilon \leq \varepsilon_{\bar{J}} \leq c_{p}(u v)$ e obtemos de (3.23) que $c_{p^{\prime}}(u v) \geq 0$, uma contradição.

Sejam $u, v \in V$ com $v \in N_{\min }(u)$. Como a condição (3.13)(iii) vale com relação a $J$ e $p$, temos $p(v) \geq p(u)$. Se $p^{\prime}(v)<p^{\prime}(u)$, concluímos de (3.22) que $u \in R$ e $v \in N_{\min }(u) \backslash R$. Mas então $p^{\prime}(v)=p(v)$ e $p^{\prime}(u)=p(u)+\varepsilon$, de modo que $\varepsilon>p(v)-p(u)$, contradizendo o fato de que $\varepsilon \leq \varepsilon_{a} \leq p(v)-p(u)$.

Além disso,

se um arco $u v \in J$ satisfaz (3.13)(i) com relação a $J$ e $p$, então também satisfaz (3.13)(i) com relação a $J$ e $p^{\prime}$.

Demonstração. Seja $u v \in J \operatorname{com} c_{p}(u v) \leq 0$. Se $c_{p^{\prime}}(u v)>0$, concluímos de (3.23) que $u v$ sai de $R$. Porém, como $\varepsilon \leq \varepsilon_{J} \leq-c_{p}(u v)$, obtemos de $(3.23)$ que $c_{p^{\prime}}(u v) \leq 0$, uma contradição.

Observe que, se $\varepsilon=\varepsilon_{b}$, então já cumprimos a nossa missão, pois o arco $b$ não viola a condição (3.13)(i) com relação a $p^{\prime}$. Prosseguimos para a iteração seguinte com $p^{\prime}$ no lugar de $p$.

Por outro lado, se $\varepsilon \neq \varepsilon_{b}$, repetimos o procedimento com $p^{\prime}$ no lugar de $p$. Observe que o conjunto de arcos induzido por $R$ é idêntico nos grafos $D^{\prime}\left(J, p^{\prime}\right)$ e $D^{\prime}(J, p)$. Ademais, como $\varepsilon \in\left\{\varepsilon_{J}, \varepsilon_{\bar{J}}, \varepsilon_{a}\right\}$, o conjunto dos vértices acessíveis a partir de $y$ em $D^{\prime}\left(J, p^{\prime}\right)$ contém $R$ propriamente. Assim, após no máximo $|V|$ repetições do procedimento acima, teremos ou $\varepsilon=\varepsilon_{b}$, ou então $x \in R$, isto é, aplicaremos o caso 2 descrito a seguir.

CASO 2: existe um caminho de $y$ a $x$ em $D^{\prime}(J, p)$, ou seja, $x \in R$.

Seja $P$ um caminho de $y$ a $x$ com o menor número possível de arcos. Note que $P$ é um caminho no grafo auxiliar $D(J, p)$ e satisfaz a condição (3.15). Assim, tomando o circuito axial $C:=P+b$ e aplicando o teorema 3.1 , obtemos uma junção $J^{\prime}$.

Como $b \notin J^{\prime}$, então $b$ não viola $(3.13)(\mathrm{i})$ com relação a $J^{\prime}$ e $p$. Observe também que os arcos de $J^{\prime} \backslash J$ satisfazem a condição (3.13)(i) com relação a $J^{\prime}$ e a $p$, pois todos os arcos de $A_{J}^{\prime}$ têm custo reduzido não-negativo. Além disso, os arcos de $J \backslash J^{\prime}$ satisfazem a condição (3.13)(ii) com relação a $J^{\prime}$ e a $p$, já que todos os arcos de $A_{\bar{J}}^{\prime}$ têm custo reduzido não-positivo. Finalmente, notamos que

$$
\text { a condição (3.13)(iii) é satisfeita por } J^{\prime} \text { e } p \text {. }
$$

Demonstração. Sejam $p_{0}, \ldots, p_{k}$ os valores distintos assumidos por $p$, com $p_{0}<\cdots<p_{k}$. Pela afirmação (3.14), basta mostrarmos que $N_{i}:=\left\{v: p(v) \geq p_{i}\right\}$ é um núcleo fechado com relação a $J^{\prime}$, para $i=1, \ldots, k$. Defina $\vec{d}_{a}(N)$ e $\overleftarrow{d}_{a}(N)$ como na demonstração do teorema 3.1 . Pela definição de $A_{a}^{\prime}$, temos $\vec{d}_{a}\left(N_{i}\right)=\overleftarrow{d}_{a}\left(N_{i}\right)=0$ para cada $i$. Assim, por (3.16), temos que $\vec{d}_{J^{\prime}}\left(N_{i}\right)=\vec{d}_{J}\left(N_{i}\right)$ para todo $i$. Agora basta invocar as afirmações (3.10) e (3.14).

Prosseguimos então para a iteração seguinte, com $J^{\prime}$ no lugar de $J$.

Isso finaliza a descrição da iteração primal-dual. 
Observe que, sempre que o caso 2 ocorre, temos $c\left(J^{\prime}\right)<c(J)$. Para ver isso, defina uma função custo $c^{\prime}: A^{\prime}(J, p) \rightarrow \mathbb{Z}$ da seguinte forma:

$$
c^{\prime}(a):= \begin{cases}-c(a), & \text { se } a \in A_{J}^{\prime}, \\ c\left(a^{-1}\right), & \text { se } a \in A_{J}^{\prime} \\ 0, & \text { se } a \in A_{a}^{\prime}\end{cases}
$$

Temos assim que $c\left(J^{\prime}\right)=c(J)+c^{\prime}(C)$, onde $C$ é o circuito axial em torno do qual giramos $J$ para obter $J^{\prime}$.

Vamos mostrar que $c^{\prime}(C)<0$. Suponha que o caminho $P$ de $y$ a $x$ usado no caso 2 foi $P=\left\langle v_{0}, a_{1}, v_{1}, \ldots, a_{k}, v_{k}\right\rangle$, ou seja, $v_{0}=y$ e $v_{k}=x$. Tome também $v_{k+1}:=y$ e $a_{k+1}:=b$. Note que $c^{\prime}(C)=\sum_{i=1}^{k+1} c^{\prime}\left(a_{i}\right)$. Se $a_{i} \in A_{J}^{\prime}$, então $c_{p}\left(a_{i}\right) \geq 0$ e portanto $c^{\prime}\left(a_{i}\right)=-c\left(a_{i}\right) \leq$ $p\left(v_{i-1}\right)-p\left(v_{i}\right)$. Se $a_{i} \in A_{J}^{\prime}$, então $c_{p}\left(a_{i}^{-1}\right) \leq 0$ e portanto $c^{\prime}\left(a_{i}\right)=c\left(a_{i}^{-1}\right) \leq p\left(v_{i-1}\right)-p\left(v_{i}\right)$. Se $a_{i} \in A_{a}^{\prime}$, então $c^{\prime}\left(a_{i}\right)=0 \leq p\left(v_{i-1}\right)-p\left(v_{i}\right)$. Finalmente, se $a_{i}=b$, então $c_{p}\left(a_{i}\right)>0 \mathrm{e}$ portanto $c^{\prime}\left(a_{i}\right)=-c\left(a_{i}\right)<p\left(v_{i-1}\right)-p\left(v_{i}\right)$. Concluímos que

$$
c^{\prime}(C)=\sum_{i=1}^{k+1} c^{\prime}\left(a_{i}\right)<\sum_{i=1}^{k+1}\left(p\left(v_{i-1}\right)-p\left(v_{i}\right)\right)=0,
$$

de modo que $c\left(J^{\prime}\right)<c(J)$.

Assim, podemos enxergar o circuito $C$ acima como uma estrutura análoga aos circuitos de custo negativo usados em algoritmos para circulação de custo mínimo. Sob essa perspectiva, é fácil ver que as condições de otimalidade (3.13) são necessárias e suficientes para que um certo supergrafo do grafo auxiliar $D(J, p)$ não tenha circuitos de custo negativo sob uma determinada função custo semelhante à dada por (3.27).

\subsection{Menores núcleos justos}

Os resultados das seções anteriores e os arcabouços apresentados no capítulo 2 sobre fluxos submodulares fornecem duas provas independentes da seguinte versão capacitada do teorema 2.3.1 de Lucchesi-Younger:

\section{Teorema 3.2 (Teorema capacitado de Lucchesi-Younger)}

Seja $D=(V, A)$ um grafo orientado e $c: A \rightarrow \mathbb{Z}_{\geq 0}$ uma função custo. Então o custo mínimo de uma junção é igual ao tamanho máximo de uma família de núcleos tal que cada arco $a$ entra em no máximo $c(a)$ desses núcleos.

Exceto pelo cômputo de $N_{\min }(v)$ para cada $v \in V$, é fácil ver que todos os demais passos do algoritmo de Frank podem ser implementados em tempo polinomial em $|V|$. Nesta seção, vamos mostrar como encontrar os conjuntos $N_{\min }(v)$ em tempo polinomial em $|V|$. Teremos assim um algoritmo fortemente polinomial para ambos os problemas de otimização do teorema capacitado de Lucchesi-Younger.

Seja $D=(V, A)$ um grafo orientado fracamente conexo, $J$ uma junção e $u \in V$. Vamos mostrar como encontrar $N_{\min }(u)$. 
Começamos observando o seguinte fato, que segue facilmente da afirmação (3.5):

um núcleo é justo se, e somente se, é uma intersecção de núcleos perigosos.

Para cada $a \in J$, denote $D_{a}:=\left(V, A \cup J_{a}^{-1}\right)$, onde $J_{a}:=J \backslash\{a\}$. Note que, para obter $D_{a}$ a partir de $D$, adicionamos o reverso de cada arco de $J$, exceto o de $a$. Abrevie $R_{a}:=\left\{v \in V: v\right.$ é acessível a partir de $u$ em $\left.D_{a}\right\}$ e tome $R:=\bigcap_{a \in J} R_{a}$. Então

$$
N_{\min }(u)=R .
$$

Demonstração. É fácil ver que, para cada $a \in J$, ou temos $R_{a}=V$, ou então $R_{a}$ é um núcleo perigoso. Como o conjunto $R$ é intersecção de núcleos perigosos contendo $u$, obtemos da afirmação (3.28) que $R$ é justo e contém $u$, de modo que $N_{\min }(u) \subseteq R$.

Seja $v \in V \backslash N_{\min }(u)$. Vamos mostrar que $v \in V \backslash R$. Seja $K$ o componente fraco de $D-N_{\min }(u)$ que contém $v$ e tome $N:=V \backslash K$. É claro que $N$ é um núcleo perigoso com $u \in N$ e $v \notin N$. Seja $a$ o único arco de $J$ que entra em $N$. É claro que $v \notin R_{a}$, de modo que $v \notin R$, como queríamos.

Concluímos que todos os passos do algoritmo descrito podem ser implementados em tempo polinomial em $n:=|V|$. Uma análise simples mostra que seu consumo de tempo é $O\left(n^{5}\right)$.

Observamos ainda que o cálculo dos conjuntos $N_{\min }(v)$ equivale, nos algoritmos para fluxos submodulares, à minimização de uma função submodular. Assim, esse passo pode ser considerado como a parte difícil do algoritmo.

\subsection{Conectores fortes de custo mínimo}

SCHRIJVER [1982] introduziu um arcabouço que fornece uma redução algorítmica de todas as relações min-max obtidas no capítulo 2 para o teorema capacitado de Lucchesi-Younger (exceto pelo teorema de intersecção de matróides de Edmonds). Nesta seção, apresentamos esse arcabouço que, junto com o algoritmo de Frank exposto neste capítulo, fornece um algoritmo fortemente polinomial para cada uma dessas relações min-max.

Sejam $D_{0}=\left(V, A_{0}\right)$ e $D=(V, A)$ grafos orientados. Dizemos que $B \subseteq A$ é um conector forte para $D_{0}$ se o grafo orientado $\left(V, A_{0} \cup B\right)$ é fortemente conexo. Dizemos que $B \subseteq A$ é um $D_{0}$-corte em $D$ se $B=\vec{\delta}_{A}(S)$ para algum subconjunto não-vazio $S \subsetneq V$ tal que $\vec{\delta}_{A_{0}}(S)=\varnothing$. É claro que um subconjunto de $A$ é um conector forte para $D_{0}$ se, e somente se, intersecta todos os $D_{0}$-cortes em $D$.

Seja $c: A \rightarrow \mathbb{Z}_{\geq 0}$ uma função custo. É fácil ver que o problema de encontrar um conector forte de custo mínimo para $D_{0}$ é NP-difícil. Vamos ver agora uma condição suficiente para que esse problema seja solúvel em tempo fortemente polinomial. Suponha que

para qualquer arco $u v \in A$, existem vértices $u^{\prime}, v^{\prime} \in V$ tais que, em $D_{0}$, o vértice $u^{\prime}$ é acessível a partir de $u$ e de $v^{\prime}$, e $v$ é acessível a partir de $v^{\prime}$.

Não é difícil ver que essa condição é satisfeita sempre que $A$ é um subconjunto de $A_{0}^{-1}$ ou que $D_{0}$ é fonte-sorvedouro-conexo. 


\section{Teorema 3.3 (Teorema do conector forte ótimo de Schrijver)}

Sejam $D_{0}=\left(V, A_{0}\right)$ e $D=(V, A)$ grafos orientados satisfazendo a condição (3.30) e seja $c: A \rightarrow \mathbb{Z}_{\geq 0}$ uma função custo. Então o custo mínimo de um conector forte para $D_{0}$ em $D$ é igual ao tamanho máximo de uma família $c$-disjunta de $D_{0}$-cortes em $D$.

Demonstração. Podemos supor que, se $u, v \in V$ e $v$ é acessível a partir de $u$ em $D_{0}$, então $u v \in A_{0}$. De fato, a adição desses arcos transitivos não altera nem os conectores fortes para $D_{0}$ em $D$ e nem os $D_{0}$-cortes em $D$.

Nossa prova é por indução em $\left|A \backslash A_{0}^{-1}\right|$. Para a base da indução, suponha que $A \subseteq A_{0}^{-1}$. É fácil ver que, nesse caso, este teorema se reduz ao teorema capacitado de Lucchesi-Younger.

Suponha então que $u v \in A \backslash A_{0}^{-1}$. Como $D_{0}$ e $D$ satisfazem a condição (3.30), existem vértices $u^{\prime}, v^{\prime} \in V$ tais que $u u^{\prime}, v^{\prime} u^{\prime}, v^{\prime} v \in A_{0}$. Tome $V^{\prime}:=V \cup\left\{u^{\prime \prime}, v^{\prime \prime}\right\}$, onde $u^{\prime \prime}$ e $v^{\prime \prime}$ são vértices novos, $A_{0}^{\prime}:=A_{0} \cup\left\{u u^{\prime \prime}, u^{\prime \prime} u^{\prime}, v^{\prime \prime} u^{\prime \prime}, v^{\prime \prime} v, v^{\prime} v^{\prime \prime}\right\}$ e $A^{\prime}:=(A \backslash\{u v\}) \cup\left\{u^{\prime \prime} v^{\prime \prime}\right\}$, sendo que $u^{\prime \prime} v^{\prime \prime}$ tem o mesmo custo que $u v$. Defina $D_{0}^{\prime}:=\left(V^{\prime}, A_{0}^{\prime}\right)$ e $D^{\prime}:=\left(V^{\prime}, A^{\prime}\right)$.

É claro que $\left|A^{\prime} \backslash A_{0}^{\prime-1}\right|<\left|A \backslash A_{0}^{-1}\right|$. Afirmamos que

$B \subseteq A$ é um conector forte para $D_{0}$ se, e somente se, $B^{\prime} \subseteq A^{\prime}$ é um conector forte para $D_{0}^{\prime}$,

onde $B^{\prime}:=(B \backslash\{u v\}) \cup\left\{u^{\prime \prime} v^{\prime \prime}\right\}$ se $u v \in B$ e $B^{\prime}:=B$ caso contrário. Isso é suficiente para provarmos o teorema. De fato, seja $B^{\prime}$ um conector forte de custo mínimo para $D_{0}^{\prime}$ em $D^{\prime}$ e $\mathcal{F}^{\prime}$ uma família de $D_{0}^{\prime}$-cortes como no enunciado. Então $\mathcal{F}:=\left\{\vec{\delta}_{A}(S \cap V): \vec{\delta}_{A^{\prime}}(S) \in \mathcal{F}\right\}$ é uma família de $D_{0}$-cortes satisfazendo a condição do enunciado, já que, se $\vec{\delta}_{A^{\prime}}(S)$ é um $D_{0^{\prime}}^{\prime}$-corte e $S$ contém $v$ mas não $u$, então $S$ contém $v^{\prime \prime}$ mas não $u^{\prime \prime}$, de modo que o arco $u^{\prime \prime} v^{\prime \prime}$, que tem o mesmo custo que $u v$, está em $\vec{\delta}_{A^{\prime}}(S)$. Assim, se provarmos a afirmação (3.31), o teorema seguirá por indução.

Seja $B \subseteq A$ um conector forte para $D_{0}$. Se $u v \notin B$, então é evidente que $B^{\prime}=B$ é um conector forte para $D_{0}^{\prime}$, já que o grafo orientado $\left(V^{\prime}, A_{0}^{\prime} \cup B^{\prime}\right)$ é obtido do grafo fortemente conexo $\left(V, A_{0} \cup B\right)$ através da adição de dois caminhos com extremidades em $V$ e do arco $v^{\prime \prime} u^{\prime \prime}$. Se $u v \in B$, então $B^{\prime}=(B \backslash\{u v\}) \cup\left\{u^{\prime \prime} v^{\prime \prime}\right\}$ também é um conector forte para $D_{0}^{\prime}$, pois todo caminho entre dois vértices de $V$ no grafo orientado $\left(V^{\prime}, A_{0} \cup B\right)$ que passa pelo arco $u v$ pode ser transformado num caminho no grafo $\left(V^{\prime}, A_{0}^{\prime} \cup B^{\prime}\right)$ com as mesmas extremidades através do atalho $u u^{\prime \prime} v^{\prime \prime} v$ entre $u$ e $v$.

Seja $B^{\prime} \subseteq A^{\prime}$ um conector forte para $D_{0}^{\prime}$. Se $u^{\prime \prime} v^{\prime \prime} \notin B^{\prime}$, então é claro que $B=B^{\prime}$ é um conector forte para $D_{0}$, já que qualquer caminho entre dois vértices de $V$ no grafo orientado $\left(V^{\prime}, A_{0}^{\prime} \cup B^{\prime}\right)$ que passa por um vértice fora de $V$ pode ser transformado num caminho no grafo $\left(V, A_{0} \cup B\right)$ com as mesmas extremidades e que evita os vértices $u^{\prime \prime}$ e $v^{\prime \prime}$. Se $u^{\prime \prime} v^{\prime \prime} \in B^{\prime}$, então $B=\left(B^{\prime} \backslash\left\{u^{\prime \prime} v^{\prime \prime}\right\}\right) \cup\{u v\}$ também é um conector forte para $D_{0}$, já que qualquer caminho entre dois vértices de $V$ no grafo $\left(V^{\prime}, A_{0}^{\prime} \cup B^{\prime}\right)$ que passa pelo arco $u^{\prime \prime} v^{\prime \prime}$ também passa, necessariamente, pelos $\operatorname{arcos} u u^{\prime \prime}$ e $v^{\prime \prime} v$, de modo que esse trecho do caminho pode ser substituído pelo arco $u v \in B$ para obtermos um caminho em $\left(V, A_{0} \cup B\right)$ com as mesmas extremidades.

Concluímos assim a prova da afirmação (3.31), e portanto do teorema. 
É claro da prova acima que qualquer algoritmo fortemente polinomial para o teorema capacitado de Lucchesi-Younger fornece um algoritmo fortemente polinomial para o teorema do conector forte ótimo de Schrijver.

Vamos ver agora alguns casos especiais desse arcabouço.

Se $D=(V, A)$ é um grafo orientado, então, tomando $A_{0}:=A^{-1}$, obtemos o teorema capacitado de Lucchesi-Younger.

Se $G$ é um grafo $(U, W)$-bipartido, então, tomando como $D$ a orientação de $G$ na qual todas as arestas são orientadas de $U$ para $W$ e $A_{0}:=\{w u: u \in U, w \in W\}$, obtemos uma versão capacitada do teorema 2.4.1 de cobertura por arestas de König-Rado.

Se $D=(V, A)$ é um grafo orientado e $r \in V$, então, tomando $A_{0}:=\{v r: v \in V\}$, obtemos o teorema 2.4.2 da arborescência ótima de Fulkerson.

Se $D=(V, A)$ é um grafo orientado e $\{R, S\}$ é uma partição de $V$, então, tomando $A_{0}:=\{s r: r \in R, s \in S\}$, obtemos o teorema 2.4.3 da bi-ramificação ótima de Schrijver.

Finalmente, se $D=(V, A)$ e $r, s \in V$, então, tomando $A_{0}:=\{u v: u=s$ ou $v=r\}$, obtemos o seguinte resultado:

\section{Corolário 3.3.1 (Teorema do potencial máximo e esforço mínimo)}

Seja $D=(V, A)$ um grafo orientado e $c: A \rightarrow \mathbb{Z}_{\geq 0}$ uma função custo. Sejam $r, s \in V$. Então o custo mínimo de um $(r, s)$-caminho é igual ao tamanho máximo de uma família $c$-disjunta de $(r, s)$-cortes.

\section{Resultados relacionados}

LUCCHESI [1976] apresentou um algoritmo polinomial para a versão não-capacitada do teorema 2.3.1 de Lucchesi-Younger, bem como um algoritmo fracamente polinomial para a versão capacitada.

FRANK [1981c] descreveu um algoritmo para intersecção de matróides com pesos que, como o algoritmo apresentado neste capítulo, é uma especialização de seu algoritmo para fluxos submodulares (veja FrANK [1982]).

FRANK E TARDOS [1984] mostraram que o problema da junção de custo mínimo pode ser reduzido ao problema de intersecção de matróides com pesos. A redução fornece também um empacotamento máximo de cortes orientados. Veja SCHRIJVER [2003, capítulo 55]. 



\section{Capítulo 4}

\section{Empacotamento de transversais de cortes}

Nos capítulos 2 e 3, derivamos diversas relações min-max que envolvem o empacotamento máximo de um certo tipo de corte e uma transversal de custo mínimo dos cortes desse tipo. Ou seja, o problema de maximização envolve o empacotamento de objetos do tipo corte e o de minimização envolve um objeto do tipo conector com custo mínimo. Neste capítulo, estudaremos relações min-max que são, num certo sentido, polares às anteriores: o problema de maximização envolve o empacotamento de objetos do tipo conector e o de minimização envolve um objeto do tipo corte de custo mínimo. Vamos ver que várias das relações derivadas anteriormente continuam valendo ao trocarmos de posição os termos referentes a objetos do tipo conector e corte.

As demonstrações que vamos apresentar são consideravelmente mais difíceis que as vistas no capítulo 2. Além disso, vamos seguir um caminho bastante diferente: em vez de provarmos um arcabouço geral e derivarmos deste certas relações min-max, vamos construir uma torre de resultados, na qual cada teorema se apóia no anterior. A base dessa torre é um celebrado teorema de Edmonds sobre o empacotamento de ramificações. Após erguermos essa torre, poderemos expor o arcabouço dos conectores fortes disjuntos de Schrijver, que contém como casos especiais todas as relações min-max da torre.

Todas as demonstrações que veremos fornecem algoritmos polinomiais para os respectivos problemas de minimização e maximização, contanto que tenhamos acesso a um algoritmo polinomial que encontra um $(r, s)$-corte mínimo. Um tal algoritmo pode ser visto em diversos livros básicos sobre algoritmos ou otimização combinatória.

O restante do capítulo está organizado da seguinte forma: na seção 4.1, provamos o teorema das ramificações disjuntas de Edmonds e derivamos algumas de suas consequiências; na seção 4.2, desviamos brevemente da construção da torre de resultados para derivar o teorema das árvores geradoras disjuntas de Tutte e Nash-Williams e o teorema de partição em florestas de Nash-Williams; na seção 4.3, retomamos nosso caminho e provamos o teorema das bi-ramificações disjuntas de Schrijver; na seção 4.4, provamos o teorema das junções disjuntas de Feofiloff-Younger e Schrijver; finalmente, na seção 4.5, expomos o teorema dos conectores fortes disjuntos de Schrijver. 


\subsection{O teorema das ramificações disjuntas de Edmonds}

Vamos começar com o seguinte lema:

\section{Lema $4.1 \alpha$}

Seja $V$ um conjunto. Seja $\mathcal{R}$ uma família de subconjuntos não-vazios de $V$. Defina as funções $\gamma: \mathcal{P}(V) \rightarrow \mathcal{R}$ e $g: \mathcal{P}(V) \rightarrow \mathbb{R}$ como $\gamma(S):=\{R \in \mathcal{R}: R \cap S=\varnothing\}$ e $g(S):=|\gamma(S)|$ para todo $S \subseteq V$. Então a função $g(S)$ é supermodular, isto é, para quaisquer $U, W \subseteq V$,

$$
g(U \cap W)+g(U \cup W) \geq g(U)+g(W) .
$$

Ademais, vale a igualdade em (4.1) se, e somente se, $\gamma(U \cap W)=\gamma(U) \cup \gamma(W)$.

Demonstração. Sejam $U, W \subseteq V$. É fácil ver que $\gamma(U \cup W)=\gamma(U) \cap \gamma(W)$ e que

$$
\gamma(U \cap W) \supseteq \gamma(U) \cup \gamma(W) .
$$

Então

$$
\begin{aligned}
g(U \cap W)+g(U \cup W) & =|\gamma(U \cap W)|+|\gamma(U \cup W)| \\
& \geq|\gamma(U) \cup \gamma(W)|+|\gamma(U) \cap \gamma(W)| \\
& =|\gamma(U)|+|\gamma(W)|=g(U)+g(W) .
\end{aligned}
$$

É evidente que essa relação vale com igualdade se, e somente se, vale a igualdade em (4.2).

Estamos prontos para provar o teorema das ramificações disjuntas de EDMONDS [1973]. Seguimos a prova de LovÁsz [1976].

Teorema 4.1 (Teorema das ramificações disjuntas de Edmonds)

Seja $D=(V, A)$ um grafo orientado e sejam $R_{1}, \ldots, R_{k}$ subconjuntos não-vazios de $V$. Então existem ramificações disjuntas $B_{1}, \ldots, B_{k}$ tais que $B_{i}$ é uma $R_{i}$-ramificação para todo $i$ se, e somente se,

$$
\vec{d}(S) \geq\left|\left\{i: R_{i} \cap S=\varnothing\right\}\right|
$$

para todo subconjunto não-vazio $S \subseteq V$.

Demonstração. A condição (4.3) é obviamente necessária. Vamos mostrar que ela também é suficiente. Para tanto, vamos construir uma ramificação de cada vez. Cada ramificação será construída arco por arco.

A prova é por indução em $\left|V \backslash R_{1}\right|+\cdots+\left|V \backslash R_{k}\right|$, tomando como base o caso trivial em que $R_{1}=\cdots=R_{k}=V$. Suponha que $R_{i} \neq V$ para algum $i$. Podemos supor que $R_{1} \neq V$. Vamos mostrar que existe um arco $x y \in \overleftarrow{\delta}\left(R_{1}\right)$ que pode ser usado para a construção de $B_{1}$. Isto é, se substituirmos $R_{1}$ por $R_{1} \cup\{y\}$ e $D$ por $D-x y$, a condição (4.3) continua válida, de modo que podemos aplicar a hipótese de indução para construir as ramificações e adicionar o arco $x y$ a $B_{1}$. 
Suponha então que $R_{1} \neq V$. Tome a família $\mathcal{R}:=\left\{R_{1}, \ldots, R_{k}\right\}$ e defina, para todo $S \subseteq V$,

$$
\gamma_{\mathcal{R}}(S):=\{R \in \mathcal{R}: R \cap S=\varnothing\}
$$

e $g_{\mathcal{R}}(S):=\left|\gamma_{\mathcal{R}}(S)\right|$. Observe que a condição (4.3) é equivalente a $\vec{d}_{D}(S) \geq g_{\mathcal{R}}(S)$ para todo subconjunto não-vazio $S \subseteq V$.

Dentre todos os conjuntos $S \subseteq V$ satisfazendo

(i) $S \cap R_{1} \neq \varnothing$,

(ii) $S \backslash R_{1} \neq \varnothing \mathrm{e}$

(iii) $\vec{d}_{D}(S)=g_{\mathcal{R}}(S)$,

seja $W$ um conjunto minimal. Note que um tal conjunto existe, pois $V$ satisfaz as condições (4.4).

É evidente que $g_{\mathcal{R}}\left(W^{\prime}\right) \geq g_{\mathcal{R}}(W)$ para todo $W^{\prime} \subseteq W$. Como temos $R_{1} \in \gamma_{\mathcal{R}}\left(W \backslash R_{1}\right)$ e $R_{1} \notin \gamma_{\mathcal{R}}(W)$, então

$$
\vec{d}_{D}\left(W \backslash R_{1}\right) \geq g_{\mathcal{R}}\left(W \backslash R_{1}\right)>g_{\mathcal{R}}(W)=\vec{d}_{D}(W) .
$$

Logo, existe um arco $a=x y \in A$ com $x \in W \cap R_{1}$ e $y \in W \backslash R_{1}$. Agora basta mostrarmos que a condição (4.3) continua valendo ao trocarmos $D$ por $D-a$ e $R_{1}$ por $R_{1} \cup\{y\}$, pois nesse caso podemos aplicar a hipótese de indução e incluir o arco $a$ em $B_{1}$.

Tome $D^{\prime}:=D-a$. Tome ainda $R_{1}^{\prime}:=R_{1} \cup\{y\}$ e $R_{i}^{\prime}:=R_{i}$ para $i=2, \ldots, k$. Abrevie $\mathcal{R}^{\prime}:=\left\{R_{1}^{\prime}, \ldots, R_{k}^{\prime}\right\}$ e defina $\gamma_{\mathcal{R}^{\prime}}(S):=\left\{R^{\prime} \in \mathcal{R}^{\prime}: R^{\prime} \cap S=\varnothing\right\}$ e $g_{\mathcal{R}^{\prime}}(S):=\left|\gamma_{\mathcal{R}^{\prime}}(S)\right|$ para todo subconjunto não-vazio $S \subseteq V$.

Suponha que a condição (4.3) não vale para o grafo $D^{\prime}$ e para os conjuntos $R_{1}^{\prime}, \ldots, R_{k}^{\prime}$, isto é, suponha que existe um subconjunto não-vazio $U \subseteq V$ tal que

$$
\vec{d}_{D^{\prime}}(U)<g_{\mathcal{R}^{\prime}}(U) .
$$

Neste caso, é claro que $\vec{d}_{D^{\prime}}(U)=\vec{d}_{D}(U)-1$ e $g_{\mathcal{R}^{\prime}}(U)=g_{\mathcal{R}}(U)$. Concluímos que $x y \in \vec{\delta}_{D}(U)$ e $\vec{d}_{D}(U)=g_{\mathcal{R}}(U)$. Como $y \in U$, então $R_{1}^{\prime} \notin \gamma_{\mathcal{R}^{\prime}}(U)$, e portanto $R_{1} \notin \gamma_{\mathcal{R}}(U)$.

Vamos mostrar agora que $U \cap W$ satisfaz as condições (4.4). Isso concluirá a prova do teorema, já que $U \cap W \subseteq W \backslash\{x\}$, contradizendo a minimalidade de $W$.

Usando a submodularidade da função $\vec{d}_{D}(S)$ e a supermodularidade da função $g_{\mathcal{R}}(S)$, provada no lema $4.1 \alpha$, obtemos que

$$
\begin{aligned}
\vec{d}_{D}(U \cap W) & \leq \vec{d}_{D}(U)+\vec{d}_{D}(W)-\vec{d}_{D}(U \cup W) \\
& \leq g_{\mathcal{R}}(U)+g_{\mathcal{R}}(W)-g_{\mathcal{R}}(U \cup W) \leq g_{\mathcal{R}}(U \cap W) .
\end{aligned}
$$

Como, por hipótese, $\vec{d}_{D}(U \cap W) \geq g_{\mathcal{R}}(U \cap W)$, segue que todas as desigualdades em (4.5) valem com igualdade, ou seja, $U \cap W$ satisfaz (4.4)(iii). Ademais, o lema $4.1 \alpha$ nos garante que $\gamma_{\mathcal{R}}(U \cap W)=\gamma_{\mathcal{R}}(U) \cup \gamma_{\mathcal{R}}(W)$. Como $R_{1} \notin \gamma_{\mathcal{R}}(U) \cup \gamma_{\mathcal{R}}(W)=\gamma_{\mathcal{R}}(U \cap W)$, então $(U \cap W) \cap R_{1} \neq \varnothing$, ou seja, $U \cap W$ satisfaz (4.4)(i). Finalmente, como $y \in U \cap W$, então $(U \cap W) \backslash R_{1} \neq \varnothing$, ou seja, $U \cap W$ satisfaz (4.4)(ii). 
Para a relação min-max a seguir, lembramos que, se $D=(V, A)$ é um grafo orientado e $r \in V$ é um vértice, então dizemos que $B \subseteq A$ é um $r$-corte se $B=\vec{\delta}(S)$ para algum subconjunto não-vazio $S \subseteq V \backslash\{r\}$.

\section{Corolário 4.1.1 (Teorema das arborescências disjuntas de Edmonds)}

Seja $D$ um grafo orientado e $r \in V_{D}$. Então o tamanho mínimo de um $r$-corte é igual ao número máximo de $r$-arborescências disjuntas.

Demonstração. Imediato do teorema 4.1 das ramificações disjuntas de Edmonds, tomando como $k$ o tamanho mínimo de um $r$-corte e $R_{i}:=\{r\}$ para $i=1, \ldots, k$.

Vale também a seguinte versão capacitada:

\section{Corolário 4.1.2}

Seja $D=(V, A)$ um grafo orientado, $c: A \rightarrow \mathbb{Z}_{\geq 0}$ uma função capacidade e $r \in V$. Então a capacidade mínima de um $r$-corte é igual ao tamanho máximo de uma família $c$-disjunta de $r$-arborescências.

Demonstração. Segue diretamente do teorema 4.1.1 das arborescências disjuntas de Edmonds, substituindo cada arco $a$ por $c(a)$ cópias paralelas do arco $a$.

Podemos derivar também uma das versões do teorema de Menger [1927]:

\section{Corolário 4.1.3 (Teorema de Menger)}

Seja $D=(V, A)$ um grafo orientado e sejam $r, s \in V$. Então o tamanho mínimo de um $(r, s)$-corte é igual ao número máximo de $(r, s)$-caminhos disjuntos nos arcos.

Demonstração. Seja $k$ o tamanho mínimo de um $(r, s)$-corte. Para cada vértice $v \in V \backslash\{r, s\}$, adicione $k$ arcos paralelos $s v$. Chame o grafo orientado resultante de $D^{\prime}$. É claro que o tamanho mínimo de um $r$-corte de $D^{\prime}$ é $k$. Pelo teorema 4.1.1 das arborescências disjuntas de Edmonds, existem $k r$-arborescências disjuntas em $D^{\prime}$. Em cada uma dessas $r$-arborescências, os arcos de um $(r, s)$-caminho estão todos contidos em $A$. Obtemos assim $k(r, s)$-caminhos disjuntos nos arcos.

Vale também a seguinte versão capacitada, obtida por DANTzIG E FulKerson [1956]:

\section{Corolário 4.1.4 (Teorema do fluxo máximo e corte mínimo)}

Seja $D=(V, A)$ um grafo orientado, $c: A \rightarrow \mathbb{Z}_{\geq 0}$ uma função capacidade e sejam $r, s \in V$. Então a capacidade mínima de um $(r, s)$-corte é igual ao tamanho máximo de uma família $c$-disjunta de $(r, s)$-caminhos.

Demonstração. Segue imediatamente do teorema 4.1.3 de Menger, substituindo cada arco $a$ por $c(a)$ cópias paralelas do arco $a$.

Veja as notas no final do capítulo para referências sobre algoritmos fortemente polinomiais para encontrar um $(r, s)$-corte de capacidade mínima. Tais algoritmos são necessários para transformar as provas dos teoremas desse capítulo em algoritmos eficientes para as respectivas relações min-max. 


\subsection{O teorema das árvores geradoras disjuntas de Tutte e Nash-Williams}

Nosso objetivo nesta seção é derivar o famoso teorema de Tutte e Nash-Williams sobre empacotamento de árvores geradoras. Para isso, usaremos o teorema 4.1.1 das arborescências disjuntas de Edmonds e um teorema de orientação de Frank.

Começamos provando o teorema de Frank [1993]. A seguinte notação será conveniente. Se $G=(V, E)$ é um grafo e $\mathcal{P}$ é uma partição de $V$, então $\delta_{G}(\mathcal{P})$ denota o conjunto de arestas de $G$ que têm suas pontas em partes diferentes de $\mathcal{P}$. Quando o grafo $G$ está claro no contexto, abreviamos $\delta(\mathcal{P}):=\delta_{G}(\mathcal{P})$.

Teorema 4.2

Seja $G=(V, E)$ um grafo, $r \in V$ e $k \in \mathbb{Z}_{\geq 0}$. Então existe uma orientação $D$ de $G$ tal que

$$
\vec{d}(S) \geq k
$$

para todo subconjunto não-vazio $S \subseteq V \backslash\{r\}$ se, e somente se,

$$
|\delta(\mathcal{P})| \geq k(|\mathcal{P}|-1)
$$

para toda partição $\mathcal{P}$ de $V$.

Demonstração. Primeiro provamos a necessidade da condição (4.7). Seja $D$ uma orientação de $G$ satisfazendo (4.6) e $\mathcal{P}$ uma partição de $V$. Para cada parte $S$ de $\mathcal{P}$ que não contém $r$, temos $\vec{d}(S) \geq k$. Assim, $|\delta(\mathcal{P})|=\sum_{S \in \mathcal{P}} \vec{d}(S) \geq k(|\mathcal{P}|-1)$.

Agora vamos provar a suficiência de (4.7). Seja $G$ um grafo que satisfaz (4.7). Dada qualquer orientação $D$ de $G$, podemos adicionar arcos artificiais da forma $r v$, com $v \in V$, possivelmente com várias cópias do arco $r v$ para um mesmo $v$, de modo que o grafo resultante satisfaça a condição (4.6). Seja $\gamma(D)$ o número mínimo de arcos artificiais que precisam ser adicionados a $D$ para que o grafo resultante satisfaça (4.6).

Seja $D$ uma orientação de $G$ que minimiza $\gamma(D)$. Vamos mostrar que $\gamma(D)=0$, ou seja, que $D$ satisfaz (4.6). Suponha então que $\gamma(D)>0$. Chame de $D^{\prime}$ o grafo obtido após a adição dos arcos artificiais. Evidentemente podemos supor que $\vec{d}_{D^{\prime}}(r)=0$.

Dizemos que um subconjunto não-vazio $S \subseteq V \backslash\{r\}$ é justo se $\vec{d}_{D^{\prime}}(S)=k$.

Seja $r w$ um arco artificial de $D^{\prime}$ e $W$ o conjunto dos vértices acessíveis a partir de $w$ em $D^{\prime}$. Suponha que existe um vértice $u \in W$ que não está em nenhum conjunto justo. É claro que $u \neq w$, pois caso contrário teríamos que $D^{\prime}-r w$ satisfaz (4.6), contradizendo a minimalidade de $\gamma(D)$. Seja $P$ um caminho que liga $w$ a $u$ em $D^{\prime}$. Inverta a orientação dos arcos de $P$ e remova o arco artificial $r w$. É fácil ver que o grafo resultante satisfaz (4.6), o que contradiz a escolha de $D$, pois o número de arcos artificiais diminuiu. Concluímos então que

$$
\text { todo vértice } u \in W \text { está em algum conjunto justo. }
$$

Nosso objetivo agora será encontrar uma partição de $V$ que viola a condição (4.7). Essa contradição finalizará a prova. Antes, vamos mostrar que,

$$
\text { se } U \text { é um conjunto justo e } U \cap W \neq \varnothing \text {, então } U \subseteq W \text {. }
$$


Suponha que $U \backslash W \neq \varnothing$ e vamos ver que isso é impossível. Tome $Z:=V \backslash W$. Então o conjunto $U \cap Z \subseteq V \backslash\{r\}$ é não-vazio e $\vec{d}_{D^{\prime}}(Z)=0$, de modo que

$$
k=\vec{d}_{D^{\prime}}(U)+\vec{d}_{D^{\prime}}(Z)=\vec{d}_{D^{\prime}}(U \cap Z)+\vec{d}_{D^{\prime}}(U \cup Z)+d_{D^{\prime}}(U, Z) \geq k
$$

onde $d_{D^{\prime}}(U, Z)$ denota o número de $\operatorname{arcos}$ de $D^{\prime}$ com uma ponta em $U \backslash Z$ e outra em $Z \backslash U$. Segue que a última relação de (4.10) vale com igualdade, de modo que $U \cap Z$ é justo, e $\vec{d}_{D^{\prime}}(U \cup Z)$ e $d_{D^{\prime}}(U, Z)$ são ambos nulos. Como $\vec{d}_{D^{\prime}}(U \cup Z)=0$, então $\overleftarrow{d}_{D^{\prime}}(W \backslash U)=0$. Logo, temos que $w \in U$, pois $U \cap W \neq \varnothing$. Mas agora temos $r \in Z \backslash U$ e $w \in U \backslash Z$, de modo que o arco artificial $r w$ contradiz o fato de que $d_{D^{\prime}}(U, Z)=0$. Isso conclui a prova de (4.9).

Seja $\mathcal{P}_{W}$ a coleção dos conjuntos justos maximais contidos em $W$. Pelas afirmações (4.8) e (4.9), temos $\bigcup \mathcal{P}_{W}=W$. Afirmamos que $\mathcal{P}_{W}$ é uma partição de $W$. De fato, suponha que $X, Y \in \mathcal{P}_{W} \operatorname{com} X \cap Y \neq \varnothing$. Então

$$
k+k=\vec{d}_{D^{\prime}}(X)+\vec{d}_{D^{\prime}}(Y) \geq \vec{d}_{D^{\prime}}(X \cap Y)+\vec{d}_{D^{\prime}}(X \cup Y) \geq k+k
$$

de modo que todas as relações em (4.11) valem com igualdade. Em particular, $X \cup Y$ é justo, e portanto $X=Y=X \cup Y$. Segue que $\mathcal{P}_{W}$ é uma partição de $W$.

Tome $\mathcal{P}:=\mathcal{P}_{W} \cup\{V \backslash W\}$. Para toda parte $S \in \mathcal{P}_{W}$, temos $\vec{d}_{D^{\prime}}(S)=k$, pois cada um desses conjuntos é justo. Como o arco artificial $r w$ entra em exatamente uma parte de $\mathcal{P}_{W}$, então $\vec{d}_{D}(S)<\vec{d}_{D^{\prime}}(S)$ para pelo menos uma parte $S$ de $\mathcal{P}_{W}$. Ademais, pela definição de $W$, temos $\vec{d}_{D^{\prime}}(V \backslash W)=0$. Concluímos assim que

$$
|\delta(\mathcal{P})|=\sum_{S \in \mathcal{P}} \vec{d}_{D}(S)<\sum_{S \in \mathcal{P}} \vec{d}_{D^{\prime}}(S)=k(|\mathcal{P}|-1)
$$

ou seja, a partição $\mathcal{P}$ viola a condição (4.7). Essa contradição finaliza a prova.

Podemos agora derivar facilmente a seguinte relação min-max, obtida independentemente por TutTe [1961a] e Nash-Williams [1961]:

\section{Corolário 4.2.1}

\section{(Teorema das árvores geradoras disjuntas de Tutte e Nash-Williams)}

Seja $G$ um grafo e $k \in \mathbb{Z}_{\geq 0}$. Então $G$ possui $k$ árvores geradoras disjuntas nas arestas se, e somente se, para toda partição $\mathcal{P}$ de $V$, vale a condição (4.7).

Demonstração. A necessidade de (4.7) é óbvia. Para provar sua suficiência, suponha que ela é válida. Fixe um vértice arbitrário $r \in V_{G}$. Pelo teorema 4.2, existe uma orientação $D$ de $G$ tal que o tamanho mínimo de um $r$-corte em $D$ é pelo menos $k$. Pelo teorema 4.1 .1 das arborescências disjuntas de Edmonds, $D$ possui $k r$-arborescências disjuntas, que fornecem $k$ árvores geradoras disjuntas nas arestas em $G$. 
Podemos também derivar a seguinte relação min-max de NASH-Williams [1964], que é um caso particular do teorema de EDmonds [1965d] sobre partição do conjunto-base de um matróide em conjuntos independentes:

\section{Corolário 4.2.2 (Teorema de partição em florestas de Nash-Williams)}

Seja $G=(V, E)$ um grafo e $k \in \mathbb{Z}_{\geq 0}$. Então $E$ pode ser particionado em $k$ florestas se, e somente se,

$$
|E[S]| \leq k(|S|-1)
$$

para todo subconjunto não-vazio $S \subseteq V$.

Demonstração. A necessidade da condição (4.12) segue facilmente do fato de que, se $F \subseteq E$ é uma floresta, então $F[S] \leq|S|-1$ para todo subconjunto não-vazio $S \subseteq V$.

Vamos provar a suficiência de (4.12). Chame um subconjunto não-vazio $S \subseteq V$ de justo (em $G$ ) se $S$ satisfaz (4.12) com igualdade. Note que todo conjunto unitário é justo.

Suponha que $V$ não é justo. Seja $u \in V$. Seja $U$ um conjunto justo maximal contendo $u$. Então $U \neq V$. Seja $w \in V \backslash U$. Afirmamos que nenhum conjunto justo contém ambos $w$ e $u$. Pois suponha que um tal conjunto existe. Chame-o de $W$. Pela submodularidade da função $d(S)$, temos

$$
\begin{aligned}
k(|U|-1)+k(|W|-1) & =|E[U]|+|E[W]|=\frac{1}{2}(g(U)-d(U)+g(W)-d(W)) \\
& \leq \frac{1}{2}(g(U \cap W)-d(U \cap W)+g(U \cup W)-d(U \cup W)) \\
& =|E[U \cap W]|+|E[U \cup W]| \\
& \leq k(|U \cap W|-1)+k(|U \cup W|-1) \\
& =k(|U|-1)+k(|W|-1),
\end{aligned}
$$

de modo que $U \cup W$ também é justo, contradizendo a maximalidade de $U$. Concluímos que nenhum conjunto justo contém ambos $w$ e $u$, de modo que o grafo $G+u w$ também satisfaz (4.12).

Repetindo o procedimento acima, podemos construir um supergrafo $H=\left(V, E_{H}\right)$ de $G$ tal que $V$ é justo em $H$. Vamos provar agora que $H$ satisfaz a condição (4.7). Seja $\mathcal{P}$ uma partição de $V$. Então $\left|\delta_{H}(\mathcal{P})\right|=\left|E_{H}\right|-\sum_{S \in \mathcal{P}}|E[S]| \geq k(|V|-1)-k \sum_{S \in \mathcal{P}}(|S|-1)=$ $k(|V|-1)-k(|V|-|\mathcal{P}|)=k(|\mathcal{P}|-1)$. Portanto, pelo teorema 4.2.1 das árvores geradoras disjuntas de Tutte e Nash-Williams, $H$ possui $k$ árvores geradoras disjuntas nas arestas. Como o conjunto $V$ é justo em $H$, então toda aresta de $H$ está em alguma dessas árvores. Assim, ao restringirmos essas árvores ao conjunto de arestas do grafo original $G$, obtemos $k$ florestas que particionam $E$, como queríamos.

O teorema de partição em florestas de Nash-Williams é a única relação min-max desse capítulo para a qual a demonstração não fornece imediatamente um algoritmo polinomial. Indicamos o livro de SchriJver [2003, capítulo 51] ao leitor interessado num tal algoritmo. 


\subsection{O teorema das bi-ramificações disjuntas de Schrijver}

Nesta seção, provamos uma relação min-max polar ao teorema 2.4 .3 da bi-ramificação ótima de Schrijver.

Um passo fundamental na prova será o de "colar" duas ramificações ao longo de um corte. Para isso, vamos mostrar uma propriedade de troca para ramificações.

\section{Lema $4.3 \alpha$ (Propriedade de troca para ramificações)}

Seja $D=(V, A)$ um grafo orientado. Suponha que $A$ pode ser particionado em $\left\{B_{1}, B_{2}\right\}$, onde $B_{i}$ é uma $R_{i}$-ramificação para $i=1,2$. Sejam $R_{1}^{\prime}, R_{2}^{\prime} \subseteq V$ subconjuntos tais que $R_{1}^{\prime} \cup R_{2}^{\prime}=R_{1} \cup R_{2}$ e $R_{1}^{\prime} \cap R_{2}^{\prime}=R_{1} \cap R_{2}$. Então $A$ pode ser particionado em $\left\{B_{1}^{\prime}, B_{2}^{\prime}\right\}$, onde $B_{i}^{\prime}$ é uma $R_{i}^{\prime}$-ramificação para $i=1,2$, se, e somente se, cada componente forte $K$ de $D$ com $\vec{d}(K)=0$ intersecta ambos $R_{1}^{\prime}$ e $R_{2}^{\prime}$.

Demonstração. Para ver que a condição é necessária, basta notar que toda ramificação tem pelo menos uma raiz em cada componente forte $K \operatorname{com} \vec{d}(K)=0$.

Para a suficiência da condição, basta provarmos que

$$
\vec{d}(S) \geq\left\{i \in\{1,2\}: R_{i}^{\prime} \cap S \neq \varnothing\right\}
$$

para todo subconjunto não-vazio $S \subseteq V$. De fato, se vale a condição (4.13), então pelo teorema 4.1 das ramificações disjuntas de Edmonds, existem ramificações disjuntas $B_{1}^{\prime}$ e $B_{2}^{\prime}$ tais que $B_{i}^{\prime}$ é uma $R_{i}^{\prime}$-ramificação para $i=1,2$. Além disso, como $\left|R_{1}^{\prime}\right|+\left|R_{2}^{\prime}\right|=\left|R_{1}\right|+\left|R_{2}\right|$, então $\left|B_{1}^{\prime}\right|+\left|B_{2}^{\prime}\right|=\left|B_{1}\right|+\left|B_{2}\right|$, de modo que $\left\{B_{1}^{\prime}, B_{2}^{\prime}\right\}$ é de fato uma partição de $A$.

Suponha então que (4.13) é violada por um subconjunto não-vazio $U \subseteq V$. Então o lado direito de (4.13) vale 1 ou 2. Se for 2 , então $U$ é disjunto de $R_{1}^{\prime} \cup R_{2}^{\prime}=R_{1} \cup R_{2}$, ou seja, $U$ é disjunto de $R_{1}$ e de $R_{2}$. Mas então temos que $\vec{d}(U) \geq 2$, uma contradição. Portanto, o lado direito de (4.13) é 1 e $\vec{d}(U)=0$. Se escolhermos um tal $U$ minimal, então $U$ é um componente forte de $D$ tal que $\vec{d}(U)=0$. Mas então, por hipótese, o lado direito de (4.13) vale 0. Essa contradição garante a validade da condição (4.13), e portanto, do lema.

Vamos relembrar as definições de $(R, S)$-bi-ramificação e $(R, S)$-bicorte.

Seja $D=(V, A)$ um grafo orientado e $\{R, S\}$ uma bipartição de $V$. Dizemos que $B \subseteq A$ é uma $(R, S)$-bi-ramificação se, no grafo orientado $(V, B)$, cada vértice de $S$ é acessível a partir algum vértice de $R$ e cada vértice de $R$ acessa algum vértice de $S$. Dizemos que $B \subseteq A$ é um $(R, S)$-bicorte se $B=\vec{\delta}(U)$ para algum subconjunto não-vazio $U \subsetneq V$ tal que $U \subseteq S$ ou $U \supseteq S$.

Podemos provar agora a seguinte relação min-max, obtida por SCHRIJVER [1982]:

\section{Teorema 4.3 (Teorema das bi-ramificações disjuntas de Schrijver)}

Seja $D=(V, A)$ um grafo orientado e $\{R, S\}$ uma bipartição de $V$. Então o tamanho mínimo de um $(R, S)$-bicorte é igual ao número máximo de $(R, S)$-bi-ramificações disjuntas.

Demonstração. Dizemos que $B \subseteq A$ é uma co-ramificação se $B^{-1}$ é uma ramificação no grafo orientado $\left(V, A^{-1}\right)$. Nesse caso, se $B^{-1}$ é uma $R$-ramificação em $\left(V, A^{-1}\right)$, dizemos que $B$ é uma $R$-co-ramificação. 
Seja $k$ o tamanho mínimo de um $(R, S)$-bicorte. Pelo teorema 4.1 das ramificações disjuntas de Edmonds, o grafo orientado $D$ possui $k R$-ramificações disjuntas, digamos, $B_{1}, \ldots, B_{k}$, e $k S$-co-ramificações disjuntas, digamos, $B_{1}^{\prime}, \ldots, B_{k}^{\prime}$. Escolha $B_{1}, \ldots, B_{k}$ e $B_{1}^{\prime}, \ldots, B_{k}^{\prime}$ de modo a minimizar a soma

$$
\sum_{i=1}^{k} \sum_{\substack{j=1 \\ j \neq i}}^{k}\left|B_{i} \cap B_{j}^{\prime}\right| .
$$

Vamos mostrar que a soma (4.14) vale 0. Note que isso conclui a prova do teorema, já que, nesse caso, $B_{1} \cup B_{1}^{\prime}, \ldots, B_{k} \cup B_{k}^{\prime}$ são $k(R, S)$-bi-ramificações disjuntas.

Suponha então que a soma (4.14) é positiva. Podemos supor, sem perda de generalidade, que $B_{1} \cap B_{2}^{\prime} \neq \varnothing$. Defina os conjuntos

$$
\begin{aligned}
X & :=\left(B_{1} \cup B_{2}\right) \cap A[S], & Y & :=\left(B_{1} \cup B_{2}\right) \cap \vec{\delta}(S), \\
X^{\prime} & :=\left(B_{1}^{\prime} \cup B_{2}^{\prime}\right) \cap A[R], & Y^{\prime} & :=\left(B_{1}^{\prime} \cup B_{2}^{\prime}\right) \cap \vec{\delta}(S) .
\end{aligned}
$$

Observe que $Y \cap Y^{\prime} \neq \varnothing$. Nosso objetivo agora será reorganizar as $(R, S)$-bi-ramificações $B_{1} \cup B_{1}^{\prime}$ e $B_{2} \cup B_{2}^{\prime}$ de modo a torná-las disjuntas. Para isso, vamos primeiro redistribuir os arcos de $Y \cup Y^{\prime}$. Depois, temos que redistribuir os arcos de $X \cup X^{\prime}$ para encaixá-los corretamente nos arcos redistribuídos de $Y \cup Y^{\prime}$, formando duas $(R, S)$-bi-ramificações disjuntas.

Começamos reorganizando $Y \cup Y^{\prime}$. Seja $\mathcal{K}$ o conjunto de componentes fortes $K$ do grafo orientado $(S, X)$ tais que $\vec{d}_{X}(K)=0$. É claro que $\vec{d}_{Y}(K)=\vec{d}_{B_{1} \cup B_{2}}(K) \geq 2$ para cada $K \in \mathcal{K}$. Seja $\mathcal{K}^{\prime}$ o conjunto de componentes fortes $K^{\prime}$ do grafo orientado $\left(R, X^{\prime}\right)$ tais que $\overleftarrow{d}_{X^{\prime}}\left(K^{\prime}\right)=0$ É claro que $\overleftarrow{d}_{Y^{\prime}}\left(K^{\prime}\right)=\overleftarrow{d}_{B_{1}^{\prime} \cup B_{2}^{\prime}}\left(K^{\prime}\right) \geq 2$ para cada $K^{\prime} \in \mathcal{K}^{\prime}$

Construa um grafo $H$ sobre $Y \cup Y^{\prime}$ com conjunto de arestas $M \cup M^{\prime}$, onde $M$ e $M^{\prime}$ são definidos a seguir. Para cada $K \in \mathcal{K}$, escolha dois $\operatorname{arcos} a, a^{\prime} \in \vec{\delta}_{Y}(K)$ e adicione a aresta $a a^{\prime}$ a $M$; para cada $K^{\prime} \in \mathcal{K}^{\prime}$, escolha dois $\operatorname{arcos} a, a^{\prime} \in \overleftarrow{\delta}_{Y^{\prime}}\left(K^{\prime}\right)$ e adicione a aresta $a a^{\prime}$ a $M^{\prime}$ Note que $M$ e $M^{\prime}$ são emparelhamentos, de modo que os componentes de $H$ são caminhos e circuitos pares. Portanto, o grafo $H$ é bipartido. Seja $\left\{V_{1}, V_{2}\right\}$ uma bipartição de $H$. Tome $Y_{i}:=Y \cap V_{i}$ e $Y_{i}^{\prime}:=Y^{\prime} \cap V_{i}$ para $i=1,2$. Obtemos assim uma bipartição $\left\{Y_{1}, Y_{2}\right\}$ de $Y$ e uma bipartição $\left\{Y_{1}^{\prime}, Y_{2}^{\prime}\right\}$ de $Y^{\prime}$ tais que

(i) $\vec{d}_{Y_{i}}(K) \geq 1$ para todo $K \in \mathcal{K}$ e $i=1,2$,

(ii) $\overleftarrow{d}_{Y_{i}^{\prime}}\left(K^{\prime}\right) \geq 1$ para todo $K^{\prime} \in \mathcal{K}^{\prime}$ e $i=1,2$,

(iii) $Y_{1} \cap Y_{2}^{\prime}=\varnothing$ e $Y_{2} \cap Y_{1}^{\prime}=\varnothing$.

Agora vamos redistribuir os arcos de $X$ de modo a formar $R$-ramificações $\tilde{B}_{1}$ e $\tilde{B}_{2}$ tais que $\tilde{B}_{i} \cap \vec{\delta}(S)=Y_{i}$ para $i=1,2$. Para cada $A^{\prime} \subseteq A$, defina $f\left(A^{\prime}\right):=\left\{v: u v \in A^{\prime}\right\}$. Vamos aplicar o lema $4.3 \alpha$ da propriedade de troca para ramificações sobre o grafo orientado $(S, X)$ com conjuntos de raízes $f\left(Y_{1}\right)$ e $f\left(Y_{2}\right)$. A relação (4.15)(i) nos garante que a condição do lema é satisfeita. Obtemos então uma bipartição $\left\{X_{1}, X_{2}\right\}$ de $X$ tal que $X_{i}$ é uma $f\left(Y_{i}\right)$-ramificação para $i=1,2$. Defina $\tilde{B}_{i}:=Y_{i} \cup X_{i}$ para $i=1,2$. Observe que $\tilde{B}_{1}$ e $\tilde{B}_{2}$ são $R$-ramificações disjuntas tais que $\tilde{B}_{1} \cup \tilde{B}_{2}=B_{1} \cup B_{2}$. 
De forma absolutamente análoga, podemos construir $S$-co-ramificações disjuntas $\tilde{B}_{1}^{\prime}$ e $\tilde{B}_{2}^{\prime}$ tais que $\tilde{B}_{i}^{\prime} \cap \vec{\delta}(S)=Y_{i}^{\prime}$ para $i=1,2$ e $\tilde{B}_{1}^{\prime} \cup \tilde{B}_{2}^{\prime}=B_{1}^{\prime} \cup B_{2}^{\prime}$.

Por (4.15)(iii), temos que $\tilde{B}_{1} \cap \tilde{B}_{2}^{\prime}=\varnothing$ e $\tilde{B}_{2} \cap \tilde{B}_{1}^{\prime}=\varnothing$. Portanto, se substituirmos as $(R, S)$-bi-ramificações $B_{1} \cup B_{1}^{\prime}$ e $B_{2} \cup B_{2}^{\prime}$ por $\tilde{B}_{1} \cup \tilde{B}_{1}^{\prime}$ e $\tilde{B}_{2} \cup \tilde{B}_{2}^{\prime}$, a soma (4.14) diminui. Essa contradição conclui a prova do teorema.

Essa relação min-max também vale em sua versão capacitada:

\section{Corolário 4.3.1}

Seja $D=(V, A)$ um grafo orientado, $c: A \rightarrow \mathbb{Z}_{\geq 0}$ uma função capacidade e $\{R, S\}$ uma bipartição de $V$. Então a capacidade mínima de um $(R, S)$-bicorte é igual ao tamanho máximo de uma família $c$-disjunta de $(R, S)$-bi-ramificações.

Demonstração. Segue diretamente do teorema 4.3 das bi-ramificações disjuntas de Schrijver, substituindo cada arco $a$ por $c(a)$ cópias paralelas do arco $a$.

\subsection{O teorema das junções disjuntas}

Nosso objetivo nesta seção é provar uma relação min-max polar à do teorema capacitado de Lucchesi-Younger (teorema 3.2), porém restrita a grafos fonte-sorvedouro-conexos. Este é um caso particular da conjectura de Edmonds-Giles, que estudaremos melhor no capítulo 5.

Como aquecimento, vamos usar o teorema 4.1.1 das arborescências disjuntas de Edmonds para provar o seguinte teorema de FrANK [1979b]:

\section{Teorema 4.4}

Seja $D=(V, A)$ um grafo orientado e $c: A \rightarrow \mathbb{Z}_{\geq 0}$ uma função capacidade. Se $D$ possui uma arborescência, então a capacidade mínima de um corte orientado é igual ao tamanho máximo de uma família $c$-disjunta de junções.

Demonstração. Suponha que $D$ possui uma $r$-arborescência para um certo $r \in V$. A prova se baseia no fato de que todo corte orientado é um $r$-corte.

Seja $k$ a capacidade mínima de um corte orientado. Para cada $\operatorname{arco} a \in A$, crie um $\operatorname{arco} a^{-1}$ com capacidade $k$. Chame o grafo resultante de $D^{\prime}=\left(V, A^{\prime}\right)$ e a função capacidade resultante de $c^{\prime}$. Note agora que a capacidade mínima de um $r$-corte de $D^{\prime}$ (com relação a $c^{\prime}$ ) é $k$. Logo, pela versão capacitada do teorema das arborescências disjuntas de Edmonds (corolário 4.1.2), $D^{\prime}$ possui $r$-arborescências $c^{\prime}$-disjuntas $B_{1}^{\prime}, \ldots, B_{k}^{\prime}$.

Tome $J_{i}:=B_{i}^{\prime} \cap A$ para $i=1, \ldots, k$. Afirmamos que $J_{i}$ é uma junção de $D$ para cada $i$. De fato, seja $\vec{\delta}_{D}(S)$ um corte orientado de $D$. Abrevie $J:=J_{i}$ e $B^{\prime}:=B_{i}^{\prime}$ para algum $i$. Então $\vec{\delta}_{A}(S)=\vec{\delta}_{A^{\prime}}(S)$, pois $\overleftarrow{\delta}_{A}(S)=\varnothing$. Logo, $\vec{\delta}_{J}(S)=\vec{\delta}_{B^{\prime}}(S) \neq \varnothing$. Assim, cada $J_{i}$ é uma junção. Ademais, cada arco $a \in A$ está em no máximo $c^{\prime}(a)=c(a)$ das junções $J_{1}, \ldots, J_{k}$, como queríamos.

A seguir, vamos mostrar que a relação min-max do teorema 4.4 também vale para grafos fonte-sorvedouro-conexos. Esse resultado foi provado independentemente por FEOFILOFF E Younger [1987] e SCHriJver [1982]. 
Seja $D=(V, A)$ um grafo orientado e $k \in \mathbb{Z}_{\geq 0}$. Dizemos que $B \subseteq A$ é uma $k$-junção de $D$ se $\vec{d}_{B}(S) \geq k$ para todo subconjunto não-vazio $S \subsetneq V$ tal que $\overleftarrow{\delta}_{D}(S)=\varnothing$.

O resultado que vamos provar se refere à partição de uma $k$-junção em $k$ junções, de onde a relação min-max seguirá facilmente. Seguimos a prova de ScHriJver [2003], que pode ser vista como uma extrapolação da prova do teorema 4.4 acima.

\section{Teorema 4.5}

Num grafo orientado fonte-sorvedouro-conexo, qualquer $k$-junção pode ser particionada em $k$ junções.

Demonstração. Escolha como contra-exemplo um grafo orientado $D=(V, A)$ e uma $k$-junção $B$ de $D$ tal que $|V|+|B|$ seja o menor possível. Note que $D$ é acíclico, já que cada componente forte pode ser contraído para um único vértice. É claro que podemos supor que, se $u, v \in V$ são vértices distintos e $v$ é acessível a partir de $u$, então $u v \in A$.

Nossa prova prossegue da seguinte maneira. Primeiro derivamos diversas propriedades do contra-exemplo minimal. Após isso, vamos particionar o conjunto de vértices em $\{R, S\}$ como um preparativo para a aplicação do teorema 4.3 das bi-ramificações disjuntas de Schrijver. Concluímos aplicando esse teorema e transformando as $k$ bi-ramificações obtidas em $k$ junções. Ou seja, seguimos a mesma abordagem da prova do teorema 4.4, substituindo o teorema 4.1.1 das arborescências disjuntas de Edmonds pelo teorema 4.3 das bi-ramificações disjuntas de Schrijver.

Começamos mostrando que,

$$
\text { se } \vec{\delta}(U) \text { é um corte orientado com } \vec{d}_{B}(U)=k \text {, então }|U| \in\{1,|V|-1\} \text {. }
$$

Pois suponha o contrário. Defina $D^{\prime}:=D / U$ e $D^{\prime \prime}:=D / \bar{U}$, onde $\bar{U}:=V \backslash U$. Observe que $D^{\prime}$ e $D^{\prime \prime}$ também são fonte-sorvedouro-conexos. Seja $B^{\prime}$ o conjunto de arcos de $B$ que têm ponta inicial em $\bar{U}$ e $B^{\prime \prime}$ o conjunto de $\operatorname{arcos}$ de $B$ que têm ponta final em $U$.

Como cada corte orientado $C^{\prime}$ de $D^{\prime}$ é um corte orientado em $D$ e $\overleftarrow{\delta}(U)=\varnothing$, então é fácil ver que $\left|B^{\prime} \cap C^{\prime}\right| \geq k$. Assim, $B^{\prime}$ é uma $k$-junção de $D^{\prime}$. Pela minimalidade de $|V|+|B|$, temos que $B^{\prime}$ pode ser particionado em $k$ junções de $D^{\prime}$, digamos, $J_{1}^{\prime}, \ldots, J_{k}^{\prime}$. Ademais, como $\vec{d}_{B}(U)=k$, então $\vec{d}_{J^{\prime}}(U)=1$ para cada $J^{\prime} \in\left\{J_{1}^{\prime}, \ldots, J_{k}^{\prime}\right\}$. Similarmente, podemos particionar $B^{\prime \prime}$ em junções $J_{1}^{\prime \prime}, \ldots, J_{k}^{\prime \prime}$ tais que $\vec{d}_{J^{\prime \prime}}(U)=1$ para todo $J^{\prime \prime} \in\left\{J_{1}^{\prime \prime}, \ldots, J_{k}^{\prime \prime}\right\}$. Podemos supor que, para cada $i$, os conjuntos $J_{i}^{\prime}$ e $J_{i}^{\prime \prime}$ têm exatamente um arco em comum: o arco que entra em $U$. Logo, os conjuntos $J_{1}^{\prime} \cup J_{1}^{\prime \prime}, \ldots, J_{k}^{\prime} \cup J_{k}^{\prime \prime}$ são disjuntos.

Afirmamos que $J_{i}^{\prime} \cup J_{i}^{\prime \prime}$ é uma junção para $D$, para $i=1, \ldots, k$. Pois suponha que $\vec{\delta}_{D}(W)$ é um corte orientado disjunto de $J_{i}^{\prime} \cup J_{i}^{\prime \prime}$. Então $U \cap W \neq \varnothing$ e $U \nsubseteq W$, pois caso contrário $\vec{\delta}(W)$ seria um corte orientado em $D^{\prime}$, de modo que um arco de $J_{i}^{\prime}$ entraria em $W$. Aplicando o mesmo argumento anterior para o grafo $D^{\prime \prime}$, concluímos que $\bar{U} \cap W \neq \varnothing$ e $\bar{U} \nsubseteq W$, ou seja, $W \nsubseteq U$ e $U \cup W \neq V$. Concluímos assim que $U$ e $W$ se cruzam.

Como $0=\overleftarrow{d}_{D}(U)+\overleftarrow{d}_{D}(W) \geq \overleftarrow{d}_{D}(U \cap W)+\overleftarrow{d}_{D}(U \cup W) \geq 0$, concluímos que $\vec{\delta}_{D}(U \cap W)$ e $\vec{\delta}_{D}(U \cup W)$ também são cortes orientados de $D$. Mas $\vec{\delta}_{D}(U \cup W)$ é um corte orientado de $D^{\prime}$, de modo que algum arco de $J_{i}^{\prime}$ entra em $U \cup W$. Além disso, $\vec{\delta}_{D}(U \cap W)$ é um corte orientado de $D^{\prime \prime}$, de modo que algum arco de $J_{i}^{\prime \prime}$ entra em $U \cap W$. Como exatamente um arco de $J_{i}^{\prime} \cap J_{i}^{\prime \prime}$ entra em $U$, então algum arco de $J_{i}^{\prime} \cup J_{i}^{\prime \prime}$ entra em $W$, uma contradição. 
Segue então que $J_{1}^{\prime} \cup J_{1}^{\prime \prime}, \ldots, J_{k}^{\prime} \cup J_{k}^{\prime \prime}$ são junções disjuntas que particionam $B$. Mas isso é uma contradição, pois $D$ e $B$ formam um contra-exemplo. Essa contradição conclui a prova da afirmação (4.16).

Seja $X$ o conjunto de fontes de $D$ e $Y$ o conjunto de sorvedouros de $D$. Vamos mostrar agora que,

$$
\text { se } u v \in B \text {, então } u \in X \text { ou } v \in Y \text {. }
$$

Pois suponha o contrário. Seja $\overrightarrow{\delta_{D}}(U)$ um corte orientado de $D$ contendo o arco $u v$. Pela afirmação (4.16), é impossível que $\vec{d}_{B}(U)=k$. Logo, $\vec{d}_{B}(U)>k$. Mas então $B^{\prime}:=B \backslash\{u v\}$ é uma $k$-junção de $D$ com tamanho menor que $|B|$. Como $|V|+|B|$ é mínimo, a $k$-junção $B^{\prime}$ pode ser particionada em $k$ junções de $D$, e portanto $B$ também o pode. Essa contradição finaliza a prova da afirmação (4.17).

Agora vamos provar que

se $u v, u^{\prime} v^{\prime} \in B$ e $v$ é acessível a partir de $u^{\prime}$, então $u^{\prime} \in X$ ou $v \in Y$.

Suponha o contrário e abrevie $a:=u v$ e $a^{\prime}:=u^{\prime} v^{\prime}$. Pela afirmação (4.17), temos $u \in X$ e $v^{\prime} \in Y$. Como $D$ é fonte-sorvedouro-conexo, então $u v^{\prime} \in A$. Ademais, como $u \in X$ e $u^{\prime} \notin X$, então $a \neq a^{\prime}$. Seja $a^{\prime \prime}:=u v^{\prime}$ um arco novo e tome $D^{\prime}:=D+a^{\prime \prime}$ e $B^{\prime}:=\left(B \backslash\left\{a, a^{\prime}\right\}\right) \cup\left\{a^{\prime \prime}\right\}$.

Afirmamos que $B^{\prime}$ é uma $k$-junção de $D^{\prime}$. De fato, seja $\vec{\delta}_{D^{\prime}}(U)$ um corte orientado de $D^{\prime}$. Se $|U| \in\{1,|V|-1\}$, então $U=\{y\}$ para algum sorvedouro $y$ ou $U=V \backslash\{x\}$ para alguma fonte $x$. Nesse caso, é fácil ver que $\vec{d}_{B^{\prime}}(U)=\vec{d}_{B}(U) \geq k$. Já se $1<|U|<|V|-1$, então a afirmação (4.16) nos garante que $\vec{d}_{B}(U)>k$. Mas então temos que $\vec{d}_{B^{\prime}}(U) \geq \vec{d}_{B}(U)-1 \geq k$, já que, se ambos $a$ e $a^{\prime}$ entram em $U$, então $u \notin U$ e $v^{\prime} \in U$, de modo que $a^{\prime \prime}$ entra em $U$. Logo, $B^{\prime}$ é uma $k$-junção de $D^{\prime}$.

Como $\left|B^{\prime}\right|<|B|$, então $B^{\prime}$ pode ser particionado em $k$ junções, digamos, $J_{1}^{\prime}, \ldots, J_{k}^{\prime}$. Suponha que $a^{\prime \prime} \in J_{1}^{\prime}$. Afirmamos agora que $J_{1}:=\left(J_{1}^{\prime} \backslash\left\{a^{\prime \prime}\right\}\right) \cup\left\{a, a^{\prime}\right\}$ é uma junção de $D$. Para provar isto, basta notarmos que, se um corte orientado $\overrightarrow{\delta_{D}}(U)$ contém $a^{\prime \prime}$, então $\overrightarrow{\delta_{D}}(U)$ também contém $a$ ou $a^{\prime}$. Pois suponha o contrário. Então $u, v \notin U$ e $u^{\prime}, v^{\prime} \in U$. Segue que $u^{\prime} \neq v$, de modo que o arco $u^{\prime} v \in A$ sai de $U$, o que é uma contradição, já que $\overrightarrow{\delta_{D}}(U)$ é um corte orientado.

Mas então $\left\{J_{1}, J_{2}^{\prime}, \ldots, J_{k}^{\prime}\right\}$ é uma partição de $B$ em $k$ junções de $D$. Essa contradição conclui a prova da afirmação (4.18).

Estamos quase prontos para aplicar o teorema das bi-ramificações disjuntas de Schrijver de forma análoga à prova do teorema 4.4. Antes, precisamos particionar $V \operatorname{em}\{R, S\}$. Defina

$$
\begin{aligned}
B^{\prime} & :=\{u v \in B: u \notin X \text { ou } v \notin Y\}, \\
D_{B} & :=\left(V, A \cup B^{\prime-1}\right), \\
R & :=\left\{v \in V: X \text { é acessível a partir de } v \text { em } D_{B}\right\}, \\
S & :=V \backslash R .
\end{aligned}
$$

É evidente que $X \subseteq R$ e $\vec{\delta}_{A}(R)=\varnothing$. É claro também que, se $u v \in \overleftarrow{\delta}_{B}(R)$, então $u \in X$ e $v \in Y$, pois do contrário teríamos $v \in R$. 
Vamos mostrar agora que $Y \subseteq S$. Suponha que $D_{B}$ tem um caminho de $Y$ para $X$. Seja $P$ um tal caminho com o menor número possível de arcos. Pela definição de $B^{\prime}$, é claro que $P$ tem pelo menos dois arcos. Sejam $v^{\prime} u^{\prime}$ e $v u$ o primeiro e o último arco de $P$, respectivamente. É claro que $v^{\prime} u^{\prime}, v u \in B^{\prime-1}$, já que $v^{\prime} \in Y$ e $u \in X$. Pela afirmação (4.17) e pela minimalidade de $P$, o caminho $P-v^{\prime}-u$ não usa nenhum arco de $B^{\prime-1}$, de modo que $v$ é acessível a partir de $u^{\prime}$ em $D$. Aplicando a afirmação (4.18), obtemos que ou $u^{\prime} \in X$ ou $v \in Y$. Mas então ou $v^{\prime} u^{\prime} \notin B^{\prime-1}$ ou $v u \notin B^{\prime-1}$. Essa contradição conclui a prova de que $Y \subseteq S$.

Resumindo, temos que

(i) $X \subseteq R$,

(ii) $Y \subseteq S$,

(iii) $\vec{\delta}_{A}(R)=\varnothing$, e

(iv) se $u v \in \overleftarrow{\delta}_{B}(R)$, então $u \in X$ e $v \in Y$.

Para cada arco $a \in A$, substitua $a$ por $k$ cópias paralelas do arco $a^{-1}$. Chame o grafo orientado resultante de $D^{\prime}=\left(V, A^{\prime}\right)$. Note que $\vec{d}_{A^{\prime} \cup B}(U) \geq k$ para cada subconjunto não-vazio $U \subsetneq V$. Então, pelo teorema 4.3 das bi-ramificações disjuntas de Schrijver, o conjunto $A^{\prime} \cup B$ pode ser particionado em $k(R, S)$-bi-ramificações, digamos, $B_{1}, \ldots, B_{k}$. Tome $J_{i}:=B_{i} \cap B$ para $i=1, \ldots, k$. Vamos mostrar que cada $J_{i}$ é uma junção de $D$. Essa contradição concluirá a prova do teorema.

Suponha que $J:=J_{i}$ não é uma junção de $D$. Seja $\overrightarrow{\delta_{D}}(U)$ um corte orientado de $D$ disjunto de $J$. Como $D$ é fonte-sorvedouro-conexo, se $U$ contém alguma fonte, então $U$ contém todos os sorvedouros. Assim, ou $U$ não contém fontes ou então $U$ contém todos os sorvedouros.

Suponha que $U$ não contém fontes. Como $U$ contém algum sorvedouro, então $U \nsubseteq R$. Além disso, como $A^{\prime} \cup J$ é uma $(R, S)$-bi-ramificação e $U \cap S \neq \varnothing$, então $\vec{\delta}_{A^{\prime} \cup J}(U \cap S) \neq \varnothing$. Por outro lado, temos que $0=\overleftarrow{d}_{A}(U)+\overleftarrow{d}_{A}(S) \geq \overleftarrow{d}_{A}(U \cap S)+\overleftarrow{d}_{A}(U \cup S) \geq 0$, de modo que $\overleftarrow{d}_{A}(U \cap S)=0$. Isso implica que $\vec{d}_{A^{\prime}}(U \cap S)=0$. Mas então algum arco $u v$ de $J$ entra em $U \cap S$. Como estamos supondo que $\vec{\delta}_{J}(U)=\varnothing$, então devemos ter $u \in U \backslash S \subseteq R$ e $v \in U \cap S \subseteq S$, ou seja, $u v \in \overleftarrow{\delta}_{A}(R)$. Pela afirmação (4.19)(iv), temos $u \in X$. Mas isso é uma contradição, pois estamos supondo que $U$ não contém fontes.

Suponha, finalmente, que $U$ contém todos os sorvedouros e pelo menos uma fonte. É claro que $X \backslash U \neq \varnothing$. Logo, $U \cup S \neq V$. Como $A^{\prime} \cup J$ é uma $(R, S)$-bi-ramificação e $S \subseteq U \cup S \neq V$, então $\vec{\delta}_{A^{\prime} \cup J}(U \cup S) \neq \varnothing$. Por outro lado, $0=\overleftarrow{d}_{A}(U)+\overleftarrow{d}_{A}(S) \geq \overleftarrow{d}_{A}(U \cap S)+\overleftarrow{d}_{A}(U \cup S) \geq 0$, de modo que $\overleftarrow{d}_{A}(U \cup S)=0$. Isso implica que $\vec{d}_{A^{\prime}}(U \cup S)=0$. Mas então algum arco $u v$ de $J$ entra em $U \cup S$. Como estamos supondo que $\vec{\delta}_{J}(U)=\varnothing$, então devemos ter $u \in R$ e $v \in S \backslash U \subseteq S \backslash Y$, ou seja, $u v \in \overleftarrow{\delta}_{A}(R)$. Pela afirmação (4.19)(iv), temos $v \in Y$. Mas isso é uma contradição, pois estamos supondo que $U$ contém todos os sorvedouros. 
Obtemos assim a seguinte relação min-max, provada independentemente por FEOFILOFF E Younger [1987] e SCHRIJVER [1982]:

Corolário 4.5.1 (Teorema das junções disjuntas de Feofiloff-Younger e Schrijver) Num grafo orientado fonte-sorvedouro-conexo, o tamanho mínimo de um corte orientado é igual ao número máximo de junções disjuntas.

Demonstração. Segue diretamente do teorema 4.5, tomando como $k$ o tamanho mínimo de um corte orientado e como $k$-junção o conjunto de arcos do grafo orientado.

Vale também a versão capacitada:

\section{Corolário 4.5.2}

Seja $D=(V, A)$ um grafo orientado e $c: A \rightarrow \mathbb{Z}_{\geq 0}$ uma função capacidade. Se o grafo $D$ é fonte-sorvedouro-conexo, então a capacidade mínima de um corte orientado é igual ao tamanho máximo de uma família $c$-disjunta de junções.

Demonstração. Adicione $c(a)$ cópias paralelas de cada arco $a \in A$ e aplique o teorema 4.5, tomando como $k$ a capacidade mínima de um corte orientado e a $k$-junção formada pelo conjunto de arcos novos.

\subsection{O teorema dos conectores fortes disjuntos de Schrijver}

Vamos relembrar algumas definições. Sejam $D_{0}=\left(V, A_{0}\right)$ e $D=(V, A)$ grafos orientados. Dizemos que $B \subseteq A$ é um conector forte para $D_{0}$ se o grafo orientado $\left(V, A_{0} \cup B\right)$ é fortemente conexo. Dizemos que $B \subseteq A$ é um $D_{0}$-corte em $D$ se $B=\vec{\delta}_{A}(S)$ para algum subconjunto não-vazio $S \subsetneq V$ tal que $\overrightarrow{\vec{\delta}_{A_{0}}}(S)=\varnothing$.

SCHRIJver [1982] derivou o seguinte arcabouço do teorema 4.5.1 das junções disjuntas:

Teorema 4.6 (Teorema dos conectores fortes disjuntos de Schrijver)

Sejam $D_{0}=\left(V, A_{0}\right)$ e $D=(V, A)$ grafos orientados. Se $D_{0}$ é fonte-sorvedouro-conexo, então o tamanho mínimo de um $D_{0}$-corte em $D$ é igual ao número máximo de conectores fortes disjuntos para $D_{0}$ em $D$.

Omitimos a prova desse teorema, pois ela é absolutamente análoga à prova do teorema 3.3 do conector forte ótimo de Schrijver, sendo que usamos o teorema 4.5.1 das junções disjuntas no lugar do teorema capacitado de Lucchesi-Younger para a base da indução.

Vamos ver agora que esse arcabouço contém quase todos os resultados provados neste capítulo.

No que se segue, seja $D=(V, A)$ um grafo orientado.

Se $r, s \in V$, então, tomando $A_{0}:=\{u v: u=s$ ou $v=r\}$, obtemos o teorema 4.1 .3 de Menger.

Se $r \in V$, então, tomando $A_{0}:=\{v r: v \in V\}$, obtemos o teorema 4.1.1 das arborescências disjuntas de Edmonds. 
Se $\{R, S\}$ é uma partição de $V$, então, tomando $A_{0}:=\{s r: r \in R, s \in S\}$, obtemos o teorema 4.3 das bi-ramificações disjuntas de Schrijver.

Se $D_{0}=\left(V, A_{0}\right)$ é um grafo orientado fonte-sorvedouro-conexo e $A \subseteq A_{0}^{-1}$, obtemos o teorema 4.5 .

Lembramos que, se $G=(V, E)$ é um grafo, então $F \subseteq E$ é uma cobertura por arestas se cada vértice de $G$ é ponta de alguma aresta de $F$. Gupta $[1967,1978]$ provou a seguinte relação min-max.

\section{Corolário 4.6.1}

Num grafo bipartido, o grau mínimo é igual ao número máximo de coberturas por arestas disjuntas.

Demonstração. Seja $G$ um grafo $(U, W)$-bipartido. Oriente todas as arestas de $U$ para $W$, obtendo o grafo orientado $D$. Tome $D_{0}:=\left(U \cup W, A_{0}\right)$, onde $A_{0}:=\{w u: u \in U$ e $w \in W\}$. Agora basta aplicar o teorema 4.6 dos conectores fortes disjuntos de Schrijver.

\section{Resultados relacionados}

Como foi mencionado no começo do capítulo, as demonstrações de todos os teoremas, com uma única exceção, fornecem algoritmos polinomiais para os respectivos problemas de minimização e maximização, contanto que tenhamos acesso a um algoritmo polinomial que encontra um $(r, s)$-corte mínimo. Existem diversos algoritmos fortemente polinomiais que encontram um $(r, s)$-corte de capacidade mínima, dentre os quais destacamos as variantes de EDMONDS E KARP [1972] e Dinits [1970] para o algoritmo de caminhos aumentadores de Ford e Fulkerson [1957] e o algoritmo push-relabel de GoldBerg [1987] e GoldBerg E TARJAN [1988]. Esses algoritmos podem ser vistos em vários livros clássicos de otimização combinatória e até mesmo em livros básicos sobre algoritmos, como por exemplo o livro de Cormen, Leiserson, Rivest e Stein [2001].

Notamos que os algoritmos fornecidos pelas provas dos teoremas são polinomiais para as versões não-capacitadas das relações min-max, mas não é trivial transformá-los em algoritmos polinomiais para as versões capacitadas. Para ver como isso pode ser feito, indicamos o livro de Schrijver [2003, capítulos 53 e 57]. Veja também a seção 5.5 .

A técnica da elegante prova de LovÁsz [1976] do teorema 4.1 das ramificações disjuntas de Edmonds já foi usada com sucesso na resolução de outros problemas. Veja, por exemplo, Okamura e Seymour [1981] e Friedman e Pippenger [1987].

Na seção 4.1, derivamos do teorema 4.1.1 das arborescências disjuntas de Edmonds uma relação min-max para o número máximo de $r$-arborescências. FrANK [1979a, 1981b] mostrou como derivar do teorema 4.1.1 das arborescências disjuntas de Edmonds relações min-max para o empacotamento de arborescências sem uma escolha prévia de raízes e para a partição do conjunto de arcos em ramificações.

FEOFILOFF [1983] também forneceu um algoritmo polinomial para o teorema 4.5.1 das junções disjuntas de Feofiloff-Younger e Schrijver. 
Descrevemos a seguir um arcabouço bastante geral obtido por SchriJver [1983b]. Seja $D=(V, A)$ um grafo orientado e $\mathcal{S}$ uma família de subconjuntos de $V$. Dizemos que $B \subseteq A$ é um corte induzido por $\mathcal{S}$ se $B=\vec{\delta}(S)$ para algum $S \in \mathcal{S}$. Uma cobertura para $\mathcal{S}$ é um conjunto de arcos que intersecta todos os cortes induzidos por $\mathcal{S}$.

\section{Teorema 4.7}

Seja $V$ um conjunto e $\mathcal{S}$ uma coleção de subconjuntos de $V$ fechada sob cruzamentos. As seguintes afirmações são equivalentes:

(i) Para todo grafo orientado $D=(V, A)$, o tamanho mínimo de um corte induzido por $\mathcal{S}$ é igual ao número máximo de coberturas disjuntas para $\mathcal{S}$.

(ii) Para todo grafo orientado $D=(V, A)$ e para toda função comprimento $\ell: A \rightarrow \mathbb{Z}_{\geq 0}$, o comprimento mínimo de uma cobertura para $\mathcal{S}$ é igual ao tamanho máximo de uma família $\ell$-disjunta de cortes induzidos por $\mathcal{S}$.

(iii) $\varnothing \in \mathcal{S}$ ou $V \in \mathcal{S}$ ou não existem conjuntos $V_{1}, \ldots, V_{5} \in \mathcal{S}$ tais que $V_{1} \subseteq V_{2} \cap V_{3}, V_{2} \cup V_{3}=V, V_{3} \cup V_{4} \subseteq V_{5}$ e $V_{3} \cap V_{4}=\varnothing$.

Esse arcabouço contém todas as relações min-max contidas no teorema 4.6 dos conectores fortes disjuntos de Schrijver, inclusive este. A prova desse resultado não é muito difícil. Porém, é bastante longa e técnica. Ela segue, de certa forma, uma estratégia semelhante à que adotamos neste capítulo.

SCHRIJVER [1985] obteve ainda um outro arcabouço, relativo a colorações supermodulares, a partir do qual é fácil derivar o teorema 4.3 das bi-ramificações disjuntas de Schrijver e alguns outros resultados. 


\section{Capítulo 5}

\section{A conjectura de Edmonds-Giles}

Em 1977, Edmonds e Giles conjecturaram que, dada uma função custo nos arcos de um grafo orientado, o custo mínimo de um corte orientado é igual ao tamanho máximo de um empacotamento de junções. Essa afirmação pode ser vista como polar a uma versão capacitada do célebre teorema de Lucchesi-Younger.

Schrijver publicou um contra-exemplo para a conjectura em 1980. Porém, na década de 80, Feofiloff, Younger e Schrijver mostraram que a conjectura vale para certas classes de grafos. Após esses resultados, a conjectura permaneceu em estado dormente por quase 20 anos, quando Cornuéjols e Guenin encontraram dois novos contra-exemplos, retomando a discussão da conjectura. Desde então, novos resultados foram encontrados.

Mesmo com os contra-exemplos conhecidos e com as provas de validade para certas classes de grafos, a conjectura de Edmonds-Giles não é considerada fechada de forma satisfatória. Uma tal forma seria caracterizar exatamente para quais grafos a conjectura é válida.

Uma questão relacionada é a conjectura de Woodall, que é a versão não-capacitada da conjectura de Edmonds-Giles: num grafo orientado, o tamanho mínimo de um corte orientado é igual ao número máximo de junções disjuntas. Sobre essa conjectura sabemos menos ainda. Nenhum contra-exemplo é conhecido e, essencialmente para todas as classes de grafos para as quais sabemos que ela é válida, também vale a conjectura de Edmonds-Giles, que é mais geral. Isso contrasta com as outras relações min-max que vimos até agora, nas quais os casos capacitado e não-capacitado são claramente equivalentes.

Neste capítulo, vamos estudar melhor essas conjecturas, os contra-exemplos conhecidos, os casos especiais em que elas valem, bem como certos problemas relacionados. Tentaremos seguir os desenvolvimentos em sua ordem cronológica.

O restante do capítulo está organizado da seguinte forma: na seção 5.1, fornecemos uma breve visão geral sobre a conjectura de Edmonds-Giles; na seção 5.2, mostramos alguns casos especiais nos quais a conjectura é válida; na seção 5.3, discutimos uma versão da conjectura obtida por dualidade planar; na seção 5.4, mencionamos desenvolvimentos recentes devidos a Cornuéjols, Guenin e Williams relacionados a uma conjectura envolvendo subestruturas proibidas; na seção 5.5, tratamos do empacotamento fracionário de junções; finalmente, na seção 5.6, provamos um resultado recente de Lee e Williams sobre a validade da conjectura para grafos orientados livres do envelope como menor. 


\subsection{Uma visão geral}

Seja $D=(V, A)$ um grafo orientado e $c: A \rightarrow \mathbb{Z}_{\geq 0}$ uma função custo. Segundo a versão capacitada do teorema de Lucchesi-Younger, vista no capítulo 3, o custo mínimo de uma junção é igual ao tamanho máximo de uma família $c$-disjunta de cortes orientados. Vimos ainda no capítulo 4 que diversas relações min-max envolvendo conector de custo mínimo e empacotamento máximo de cortes continua valendo após trocarmos esses termos de posição. EDMONDS E GILES [1977] conjecturaram o seguinte:

\section{Conjectura 5.1 (Conjectura de Edmonds-Giles)}

Seja $D=(V, A)$ um grafo orientado e $c: A \rightarrow \mathbb{Z}_{\geq 0}$ uma função capacidade. Então a capacidade mínima de um corte orientado é igual ao tamanho máximo de uma família $c$-disjunta de junções.

Sabemos que a conjectura de Edmonds-Giles é falsa: SchriJver [1980] construiu um contra-exemplo, que descrevemos a seguir. Seja $D=(V, A)$ o grafo orientado da figura 5.1. Defina uma função capacidade $c: A \rightarrow \mathbb{Z}_{\geq 0}$ na qual cada arco fino tem capacidade 0 e cada arco grosso tem capacidade 1 . Denote o conjunto de arcos grossos por $B$.

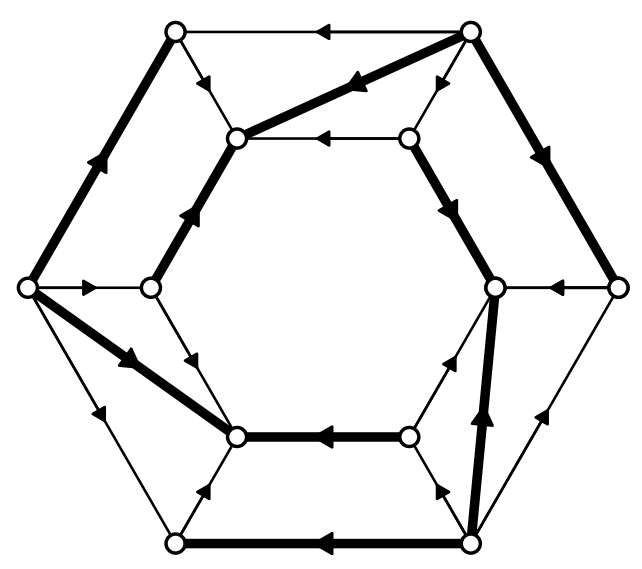

Figura 5.1: O contra-exemplo de Schrijver.

Afirmamos que a capacidade mínima de um corte orientado é 2. Para ver isso, comece verificando que $B \backslash\{a\}$ é uma junção para qualquer arco $a \in B$. Seja $\vec{\delta}(S)$ um corte orientado de $D$. Então existe um arco $a$ em $\vec{\delta}(S) \cap B$. Como $B \backslash\{a\}$ é uma junção, existe um arco $b$ em $\vec{\delta}(S) \cap(B \backslash\{a\})$. Assim, $c(\vec{\delta}(S)) \geq 2$. Ademais, $\vec{d}_{B}(s)=2$ para todo sorvedouro $s$ de $D$. Portanto, a capacidade mínima de um corte orientado é 2 .

Suponha que a conjectura de Edmonds-Giles vale para o grafo orientado $D$ com a função capacidade $c$. Então existem duas junções disjuntas contidas em $B$, digamos, $J_{1}$ e $J_{2}$. Observe que $(V, B)$ consiste de três pseudocaminhos de comprimento 3 e que cada vértice interno desses pseudocaminhos é uma fonte ou um sorvedouro. Portanto, arcos consecutivos de cada pseudocaminho devem estar em junções diferentes. 
Seja $P$ o pseudocaminho que passa pelo arco grosso mais inferior da figura 5.1. Suponha, sem perda de generalidade, que o arco do meio de $P$ está em $J_{1}$ e os arcos das pontas de $P$ estão em $J_{2}$. Agora existem 4 formas de distribuir os arcos dos outros dois pseudocaminhos entre as junções $J_{1}$ e $J_{2}$. Como pode ser visto na figura 5.2, em cada uma delas, existe um corte orientado disjunto de $J_{1}$ ou de $J_{2}$. Essa contradição nos permite concluir que esse grafo é de fato um contra-exemplo para a conjectura de Edmonds-Giles.
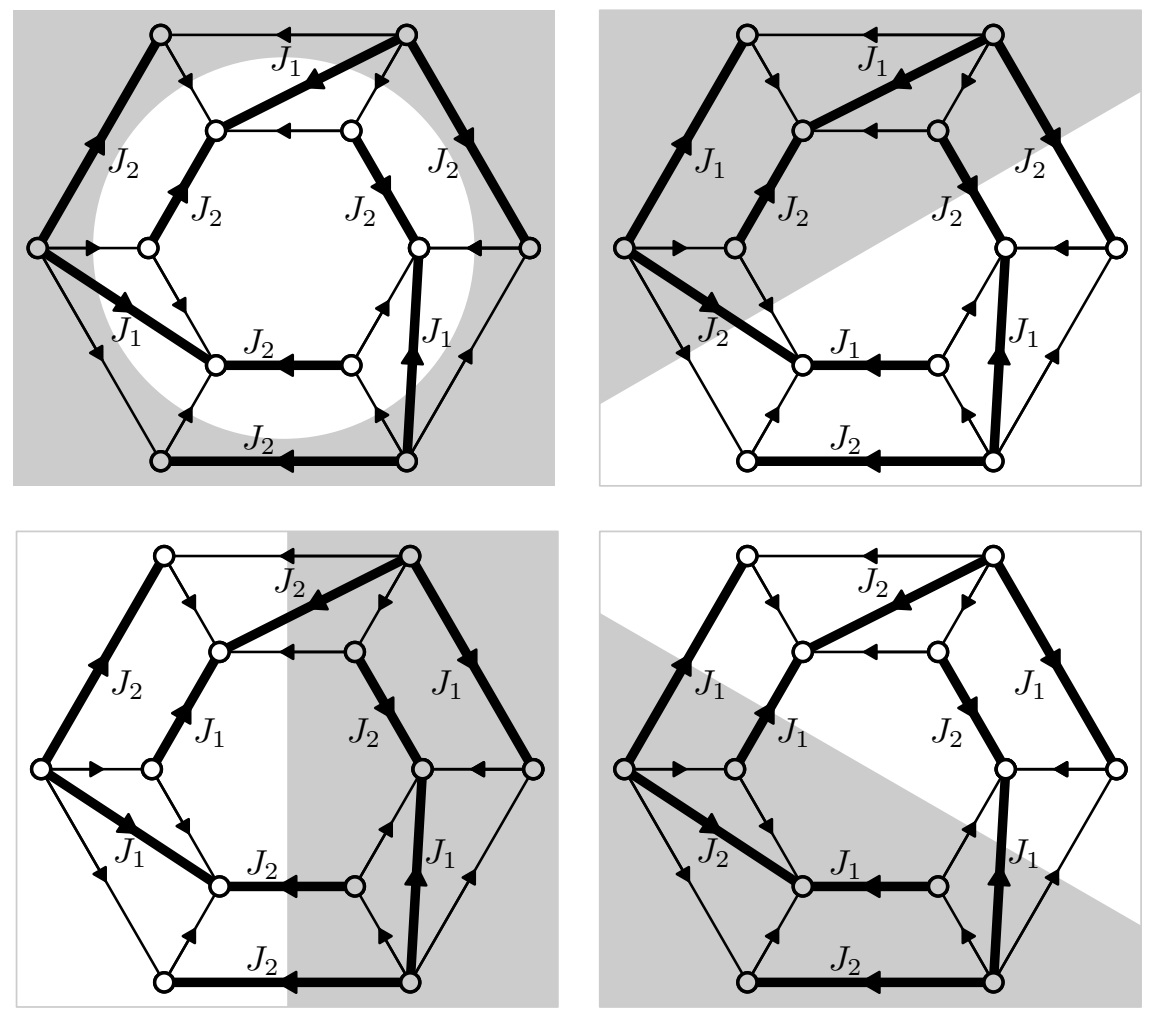

Figura 5.2: Cortes orientados não cobertos por $J_{1}$ ou $J_{2}$.

$\mathrm{Na}$ verdade, esse contra-exemplo de Schrijver faz parte de uma família infinita de contraexemplos, nos quais os pseudocircuitos interno e externo têm comprimento $2(2 k+1)$, com $k \geq 1$. Os contra-exemplos dessa família com $k=2$ e $k=3$ estão ilustrados na figura 5.3.

Vamos ver que todo membro dessa família é um contra-exemplo para a conjectura de Edmonds-Giles. Chame de $B$ o conjunto de arcos grossos. Da mesma forma como antes, é fácil ver que a capacidade mínima de um corte orientado é 2. Suponha então que existem junções disjuntas $J_{1}, J_{2} \subseteq B$. Então os arcos de cada um dos pseudocaminhos formados pelos arcos de $B$ devem ser distribuídos entre $J_{1}$ e $J_{2}$ de modo que arcos consecutivos estejam em junções diferentes. 

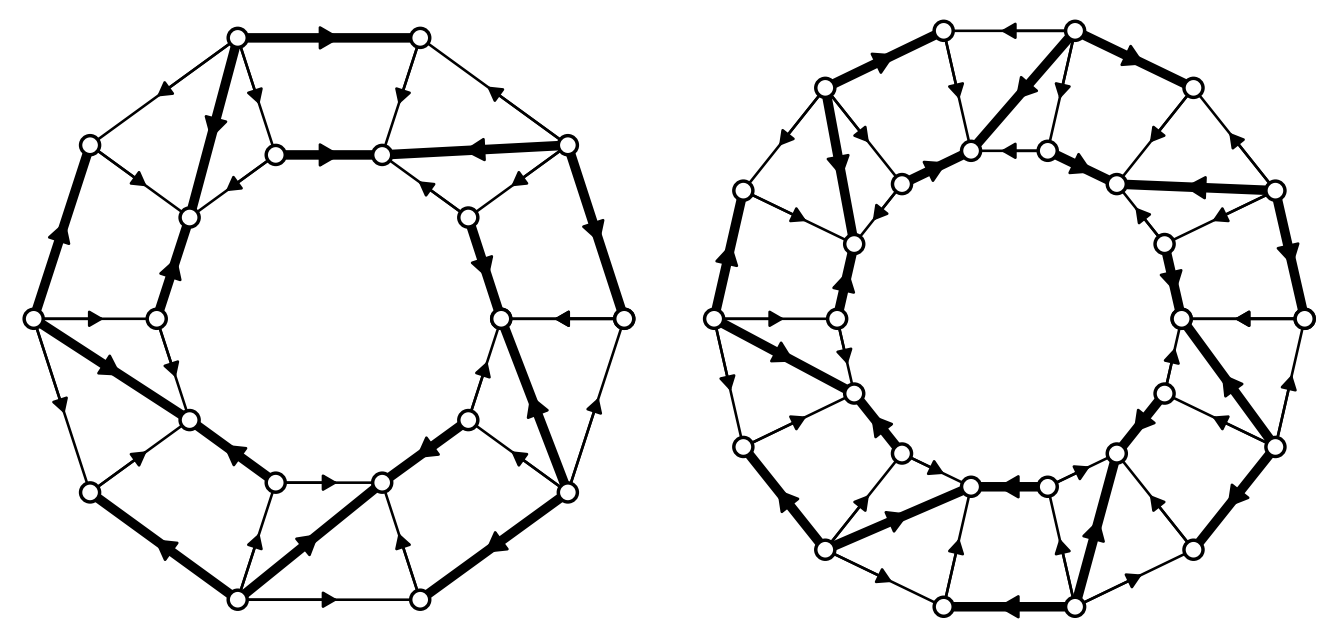

Figura 5.3: O segundo e terceiro membro da família infinita de contra-exemplos de Schrijver.

Seguindo a figura 5.4, vamos estabelecer um pouco de terminologia. Dois arcos de $B$ são chamados de correspondentes se formam lados correspondentes dos polígonos interno e externo, como é o caso dos $\operatorname{arcos} a$ e $a^{\prime}$. Um arco de $B$ é um arco de transferência se vai do polígono externo para o interno, como o arco $b$. Dois pseudocaminhos de $(V, B)$ são chamados de consecutivos se possuem arcos correspondentes. Para $i=1,2$, um pseudocaminho de $(V, B)$ é do tipo $i$ se seu arco de transferência está em $J_{i}$.

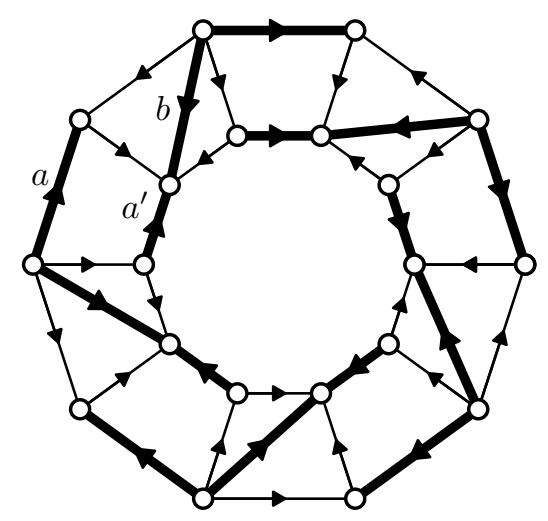

Figura 5.4: Terminologia para os contra-exemplos.

Suponha que dois pseudocaminhos consecutivos sejam do mesmo tipo, digamos, do tipo 1. Então eles possuem dois arcos correspondentes em $J_{2}$. Neste caso, é fácil encontrar cortes orientados que forcem todos os arcos de transferência a estarem em $J_{1}$. Mas então o corte orientado que separa o circuito externo do interno é disjunto de $J_{2}$, uma contradição. Logo, pseudocaminhos consecutivos são de tipos diferentes. Mas isso é impossível, já que existe um número ímpar de pseudocaminhos organizados de forma cíclica. Concluímos que cada membro dessa família infinita é um contra-exemplo para a conjectura de Edmonds-Giles. 
Note que essa prova também serve para o contra-exemplo com $k=1$. Porém, a primeira prova que mostramos também serve para outros contra-exemplos, que veremos mais adiante.

De uma perspectiva algorítmica, é fácil encontrar em tempo fortemente polinomial um corte orientado de capacidade mínima num grafo orientado: basta adicionar o reverso de cada arco, atribuindo-lhe capacidade infinita, e aplicar no grafo resultante um algoritmo de $(r, s)$-corte de capacidade mínima para cada par de vértices $r$ e $s$.

Não se sabe como encontrar um empacotamento máximo de junções em tempo polinomial, mesmo no caso não-capacitado. É possível que esse problema seja NP-difícil, mas não há evidências nessa direção.

Passemos agora ao caso não-capacitado. Woodall [1978] propôs a seguinte conjectura:

\section{Conjectura 5.2 (Conjectura de Woodall)}

Num grafo orientado, o tamanho mínimo de um corte orientado é igual ao número máximo de junções disjuntas.

Como em quase todas as relações min-max que vimos anteriormente o caso capacitado e o não-capacitado são equivalentes, é natural pensar que a conjectura de Woodall é falsa já que a conjectura de Edmonds-Giles é falsa. Em muitas relações min-max, uma construção elementar serve para provar a versão capacitada a partir da não-capacitada. Por exemplo, podemos substituir um arco de capacidade positiva por várias cópias paralelas do mesmo arco ou por um caminho, e remover ou contrair arcos de capacidade nula. Vamos tentar seguir uma abordagem semelhante para tentar mostrar que a conjectura de Woodall implica a conjectura de Edmonds-Giles.

Para cada arco $a$ com capacidade $c(a) \geq 1$, substitua o arco $a$ por $c(a)$ cópias paralelas desse arco. A intenção dessa construção é fazer com que a capacidade de cada corte orientado do grafo original seja igual ao tamanho do corte orientado equivalente no grafo derivado e permitir que um arco $a$ apareça em $c(a)$ junções diferentes. O problema é: o que fazer com os arcos de capacidade 0? As duas opções óbvias são contrair ou remover tais arcos. Porém, se contrairmos um arco, podemos destruir alguns cortes orientados, ou seja, pode ser que um corte orientado do grafo original não corresponda a nenhum corte orientado do grafo derivado. Por outro lado, se removermos um arco, podemos introduzir novos cortes orientados, ou seja, pode ser que um corte orientado do grafo obtido não corresponda a nenhum corte orientado do grafo original. Na verdade, é fácil provar que a remoção de um arco não cria novos cortes orientados se, e somente se, o arco é transitivo.

Assim, essa tentativa ingênua de mostrar que a conjectura de Woodall implica a conjectura de Edmonds-Giles está fadada ao fracasso. Não sabemos então se as duas conjecturas são equivalentes. Na verdade, a conjectura de Woodall continua em aberto.

Isso descarta a possibilidade de usarmos o seguinte argumento indutivo. Encontre uma junção $J$ que "deixe espaço suficiente" para que existam $k-1$ junções disjuntas nos arcos restantes, onde $k$ é o tamanho mínimo de um corte orientado. Agora "proíba" que os arcos de $J$ estejam nas outras $k-1$ junções (o que equivale a atribuir capacidade 0 a eles) e encontre indutivamente $k-1$ junções disjuntas que, com $J$, formarão um empacotamento de tamanho $k$. É claro que, se um argumento desse tipo funcionasse, valeria a conjectura de Edmonds-Giles. Para abordarmos a conjectura de Woodall, então, parece ser necessário encontrarmos todas as $k$ junções disjuntas de uma vez só, uma tarefa extremamente difícil. 


\section{$5.2 \quad$ Alguns casos especiais}

No capítulo 4, provamos o teorema das junções disjuntas de Feofiloff-Younger e Schrijver, que mostra que a conjectura de Edmonds-Giles vale para grafos fonte-sorvedouro-conexos. Nesta seção, vamos ver mais alguns casos especiais em que as conjecturas são válidas.

Começamos com um resultado de Schrijver [1982]. Seja $D_{0}$ um grafo orientado. Dizemos que um grafo orientado é uma extensão transitiva de $D_{0}$ se pode ser obtido a partir de $D_{0}$ através da adição de arcos transitivos em $D_{0}$.

\section{Teorema 5.3}

Seja $D=(V, A)$ uma extensão transitiva de uma árvore orientada e $c: A \rightarrow \mathbb{Z}_{\geq 0}$ uma função capacidade. Então a capacidade mínima de um corte orientado é igual ao tamanho máximo de uma família $c$-disjunta de junções.

Demonstração. Vamos seguir a estratégia sugerida no fim da seção 5.1: queremos encontrar uma junção que "deixe espaço suficiente" para encontrarmos as outras junções indutivamente. Seja $k$ a capacidade mínima de um corte orientado. Prosseguimos por indução em $k$, tomando como base o caso trivial em que $k=0$.

Chame uma junção $J$ de admissível se $\chi^{J} \leq c$, isto é, se $J$ não usa arcos de capacidade 0 . Chame $J$ de adequada se $\left(c-\chi^{J}\right)(\vec{\delta}(S)) \geq k-1$ para todo corte orientado $\vec{\delta}(S)$. Essa última condição exige que a junção $J$ deixe espaço suficiente para as outras junções. Observe que, se encontrarmos uma junção admissível e adequada, poderemos aplicar a hipótese de indução usando a função capacidade $c-\chi^{J}$ e com isso concluir a prova.

Seja $T$ uma árvore orientada da qual $D$ é uma extensão transitiva. Seja $N$ a matriz de rede gerada por $T$ e $D$. Não é difícil ver que as linhas de $N$ são exatamente os vetores de incidência dos cortes orientados minimais de $D$.

Considere o sistema de inequações lineares $\mathbf{0} \leq x \leq c, N x \geq \mathbf{1}, N(c-x) \geq(k-1) \mathbf{1}$. Escreva esse sistema como $A x \leq b$. Se existe um vetor integral $x$ satisfazendo esse sistema, então existe uma junção $J$ satisfazendo $\chi^{J} \leq x \leq c$, de modo que $J$ é admissível e adequada. Então é suficiente encontrarmos um vetor integral $x$ tal que $A x \leq b$. Mas como $N$ é totalmente unimodular (teorema 2.1), então $A$ também o é, e portanto basta que exista algum vetor $x$ tal que $A x \leq b$. Mas tal vetor existe: tome $x:=(1 / k) c$. Como $N c \geq k \mathbf{1}$, então $N x \geq \mathbf{1}$ e $N(c-x)=(k-1)(1 / k) N c \geq(k-1) \mathbf{1}$.

Vamos ver agora um caso em que a conjectura de Woodall vale. Antes precisamos de algumas definições e resultados auxiliares.

Seja $G=(V, E)$ um grafo. Uma orelha de $G$ é um caminho $P=v_{0} \cdots v_{k}$ tal que $k \geq 1$ e $V \cap V_{P}=\left\{v_{0}, v_{k}\right\}$, ou então um circuito $C=v_{0} \cdots v_{k}$ tal que $V \cap V_{C}=\left\{v_{0}\right\}$.

Um grafo $G$ possui uma decomposição em orelhas se existem grafos $G_{0}, \ldots, G_{k}$ tais que $G_{0}$ é um circuito, $G_{k}=G$ e, para $i=0, \ldots, k-1$, temos que $G_{i+1}=G_{i} \cup O_{i}$, onde $O_{i}$ é uma orelha de $G_{i}$. 


\section{Lema 5.4 $\alpha$ (Decomposição em orelhas de grafos 2-aresta-conexos)}

Um grafo admite uma decomposição em orelhas se, e somente se, é 2-aresta-conexo.

Demonstração. É evidente que qualquer grafo que admite uma decomposição em orelhas é 2-aresta-conexo. Seja $G=(V, E)$ um grafo 2-aresta-conexo. Seja $H$ um subgrafo maximal de $G$ que admite uma decomposição em orelhas. Note que tal subgrafo está bem definido, já que $G$ obviamente possui um circuito. Afirmamos que $H=G$. Pois suponha que $H \neq G$. Claramente, $H$ não pode ser gerador, pois cada aresta de $E_{G} \backslash E_{H}$ dá origem a uma orelha de $H$. Então seja $v$ um vértice de $V_{G} \backslash V_{H}$ que é vizinho de algum vértice de $H$, digamos, $w$. Como $G$ é 2 -aresta-conexo, existe um caminho $P$ de $v$ para qualquer vértice de $H$ em $G-v w$. Escolha um tal $P$ com o menor número possível de arestas. Mas então $P+v w$ é uma orelha de $H$, contradizendo a maximalidade de $H$.

Agora é fácil derivar o seguinte resultado de RoBBins [1939]:

\section{Lema $5.4 \beta$}

Um grafo admite uma orientação fortemente conexa se, e somente se, é 2-aresta-conexo.

Demonstração. A necessidade da condição é evidente. A suficiência segue imediatamente do lema $5.4 \alpha$, orientando o circuito inicial e cada orelha da forma óbvia.

O seguinte resultado é considerado parte do folclore (SCHRIJver [2003] o atribui a Frank).

\section{Teorema 5.4}

Existem 2 junções disjuntas em todo grafo orientado em que o tamanho mínimo de um corte orientado é 2 .

Demonstração. Suponha que, num grafo orientado $D=(V, A)$, o tamanho mínimo de um corte orientado é 2. Seja $G$ o grafo subjacente a $D$. É óbvio que $G$ é 2-aresta-conexo. Pelo lema $5.4 \beta$, existe uma orientação fortemente conexa $D^{\prime}$ de $G$. Defina $J_{=}:=A \cap A_{D^{\prime}}$ e $J_{\neq}:=A \backslash J_{=}$, ou seja, particionamos os arcos de $D$ em $\left\{J_{=}, J_{\neq}\right\}$, sendo que um arco fica em $J_{=}$exatamente quando tem a mesma orientação em $D$ e em $D^{\prime}$.

Seja $\overrightarrow{\delta_{D}}(S)$ um corte orientado de $D$. Como $D^{\prime}$ é fortemente conexo, então $\vec{\delta}_{D^{\prime}}(S) \neq \varnothing$, de modo que algum arco de $J_{=}$entra em $S$. Além disso, $\overleftarrow{\delta}_{D^{\prime}}(S) \neq \varnothing$, de modo que algum arco de $J_{\neq}$entra em $S$. Segue que $J_{=}$e $J_{\neq}$são junções.

Este é o único resultado que sabemos sobre a conjectura de Woodall que não se aplica também à conjectura de Edmonds-Giles. 


\subsection{A conjectura dual planar}

Uma elegante teoria sobre dualidade no plano nos fornece novas perspectivas sobre diversos resultados. Vamos ver um exemplo usando o teorema de Lucchesi-Younger.

Seja $D=(V, A)$ um grafo orientado. Dizemos que $Q \subseteq A$ é um quebra-circuitos se $D-Q$ é acíclico.

\section{Teorema 5.5 (Teorema planar de Lucchesi-Younger)}

Num grafo orientado planar, o tamanho mínimo de um quebra-circuitos é igual ao número máximo de circuitos disjuntos nos arcos.

Demonstração. Segue imediatamente do teorema 2.3.1 de Lucchesi-Younger, já que circuitos e cortes orientados minimais são trocados no grafo orientado dual (proposição 1.1).

Essa relação min-max também vale em sua versão capacitada, que omitimos.

Uma tentativa natural de extensão desse resultado seria remover a hipótese de planaridade. Porém, essa hipótese não pode ser removida, como mostra a seguinte orientação do $K_{3,3}$ : oriente todos os arcos de uma classe de cores para a outra, e depois inverta a orientação de um conjunto de arcos que forma um emparelhamento perfeito. Nesse grafo, é fácil ver que o tamanho mínimo de um quebra-circuitos é 2 e que não há 2 circuitos disjuntos nos arcos.

Voltando à conjectura de Edmonds-Giles, a dualidade no plano nos fornece a seguinte conjectura:

\section{Conjectura 5.6 (Conjectura planar de Edmonds-Giles)}

Seja $D=(V, A)$ um grafo orientado planar e $w: A \rightarrow \mathbb{Z}_{\geq 0}$ uma função peso. Então o peso mínimo de um circuito é igual ao tamanho máximo de uma família $w$-disjunta de quebracircuitos.

Como o contra-exemplo de Schrijver é planar, então seu dual fornece um contra-exemplo para essa conjectura. Porém, se dualizarmos a conjectura de Woodall, o contra-exemplo de Schrijver não se aplica:

\section{Conjectura 5.7 (Conjectura planar de Woodall)}

Num grafo orientado planar, o comprimento mínimo de um circuito é igual ao número máximo de quebra-circuitos disjuntos.

A hipótese de planaridade não pode ser removida dessa conjectura: segundo DonADELLI E KoHAYAKAWA [2002], Thomassen construiu um contra-exemplo não-planar para a afirmação da conjectura planar de Woodall. O contra-exemplo é o torneio $T$ descrito a seguir. Tome $V_{T}:=X \cup Y \cup Z$, onde $X:=\left\{x_{1}, \ldots, x_{5}\right\}, Y:=\left\{y_{1}, \ldots, y_{5}\right\}$ e $Z:=z_{1}, \ldots, z_{5}$ são disjuntos. O subgrafo induzido por $X$ tem os seguintes arcos:

$$
\begin{aligned}
& x_{1} x_{2}, x_{2} x_{3}, x_{3} x_{1}, \\
& x_{1} x_{4}, x_{4} x_{5}, x_{5} x_{1}, \\
& x_{2} x_{5}, x_{5} x_{3}, x_{3} x_{4}, x_{4} x_{2} .
\end{aligned}
$$


Os arcos induzidos por $Y$ e $Z$ são análogos. Resta descrevermos os arcos entre os conjuntos $X, Y$ e $Z$. Para $i=1, \ldots, 5$, crie $\operatorname{arcos} y_{i} x_{i}, x_{i} z_{i}, z_{i} y_{i}$. Para $i, j \in\{1, \ldots, 5\}$ distintos, crie $\operatorname{arcos} x_{i} y_{j}, y_{i} z_{j}, z_{i} x_{j}$. É claro que o comprimento mínimo de um circuito em $T$ é 3 , mas não existem 3 quebra-circuitos disjuntos. Para provar isso, Donadelli e Kohayakawa afirmam que "uma análise tediosa de casos mostra que, para destruir todos os circuitos de $T$, precisamos remover no mínimo $20+5+5+3+3+3=39>35=105 / 3$ arcos, de um total de 105 arcos". Logo, todo quebra-circuitos de $T$ tem mais de um terço do total de $\operatorname{arcos}$ de $T$, de modo que não pode haver 3 quebra-circuitos disjuntos. Infelizmente, não conseguimos reproduzir tal análise de casos, nem encontrar outro argumento para provar essa afirmação.

Donadelli E Kohayakawa [2002], usando uma versão esparsa do lema de regularidade de Szemerédi [1978] (veja os livros de Bollobás [1998] e Diestel [2005]), descoberta independentemente por Kohayakawa e Rödl (veja, por exemplo, KoHAYAKAWA [1997]), provaram o seguinte teorema:

\section{Teorema 5.8}

Seja $\ell \geq 3$ um inteiro e $\beta>0$ um real. Então para qualquer inteiro $n$ suficientemente grande, existe um grafo orientado simples $D$ com $n$ vértices e $O\left(n^{1+1 /(\ell-1)}\right)$ arcos tal que o comprimento mínimo de um circuito é $\ell$ e qualquer conjunto de $\operatorname{arcos} B \subseteq A_{D}$ tal que $|B| \geq(1 / 2+\beta)\left|A_{D}\right|$ induz um circuito de comprimento $\ell$.

Como conseqüência, eles provaram que existem infinitos grafos orientados, com densidade arbitrariamente pequena, que mostram que a hipótese de planaridade não pode ser removida da conjectura planar de Woodall:

\section{Corolário 5.8.1}

Para qualquer inteiro $\ell \geq 3$, existem infinitos grafos orientados simples com densidade arbitrariamente pequena tais que o comprimento mínimo de um circuito é $\ell$ e o número máximo de quebra-circuitos disjuntos é estritamente menor que $\ell$.

Demonstração. Seja $\varepsilon>0$. Tome $\beta:=1 / 2-1 / \ell$. Escolha $n$ suficientemente grande para que o grafo orientado $D$ devolvido pelo teorema 5.8 tenha densidade no máximo $\varepsilon$. Abrevie $m:=\left|A_{D}\right|$.

Suponha que existem $\ell$ quebra-circuitos disjuntos. Então existe um quebra-circuitos $Q$ $\operatorname{com}|Q| \leq m / \ell$. Tome $D^{\prime}:=D-Q$. Então $\left|A_{D^{\prime}}\right| \geq(1-1 / \ell) m=(1 / 2+\beta) m$ de modo que, pelo teorema 5.8, o grafo orientado $D^{\prime}$ possui um circuito, o que contradiz nossa suposição de que $Q$ é um quebra-circuitos.

A conjectura planar de Edmonds-Giles nos fornece uma nova perspectiva para a conjectura de Edmonds-Giles restrita a grafos planares, pois podemos usar propriedades de circuitos para abordar o problema. Na seção 5.6, vamos provar a conjectura planar de Edmonds-Giles para grafos orientados planares livres de $K_{5}-e$ como menor, onde $K_{5}-e$ denota o grafo obtido do $K_{5}$ após a remoção de uma aresta. Com isso, obteremos via dualidade no plano que a conjectura de Edmonds-Giles vale para grafos orientados planares livres do envelope como menor. 


\subsection{Uma conjectura envolvendo subestruturas proibidas}

Cornú́Jols e Guenin [2002] encontraram dois novos contra-exemplos para a conjectura de Edmonds-Giles, retomando a discussão sobre essa conjectura após quase duas décadas sem publicações a respeito. Os contra-exemplos estão ilustrados na figura 5.5. Como nas figuras anteriores, arcos finos têm capacidade 0 e arcos grossos têm capacidade 1 .

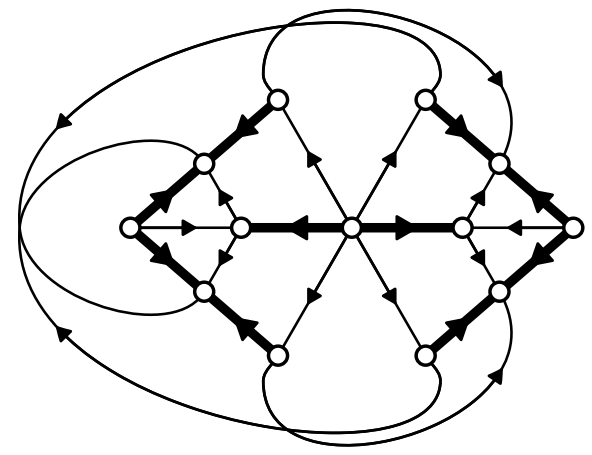

(a)

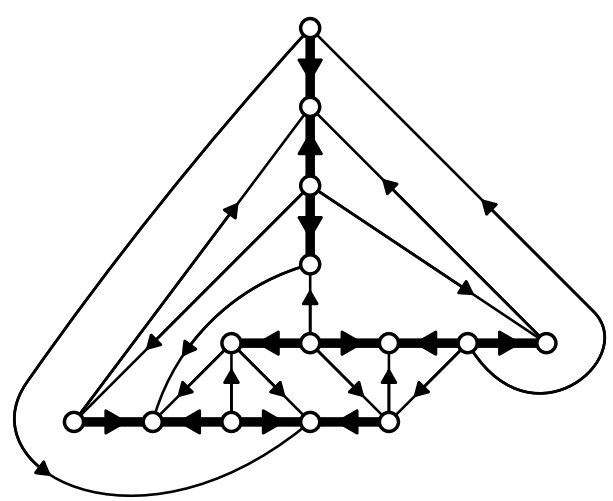

(b)

Figura 5.5: Os contra-exemplos de Cornuéjols e Guenin.

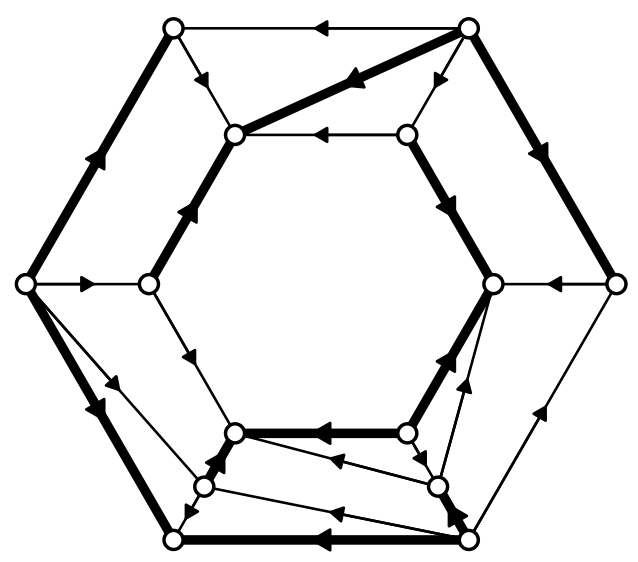

Figura 5.6: Uma nova imersão do segundo contra-exemplo de Cornuéjols e Guenin.

A prova de que esses grafos são de fato contra-exemplos pode ser feita de maneira idêntica à nossa primeira prova sobre o contra-exemplo de Schrijver: basta mostrar que, para toda forma de particionar os arcos dos pseudocaminhos formados por arcos grossos em 2 junções, sempre resta um corte orientado que não é coberto por uma das junções. 
O grafo orientado da figura 5.5(a) não é planar: é fácil encontrar um $K_{3,3}$ como menor nesse grafo. Já o grafo orientado da figura 5.5(b) é bastante similar ao contra-exemplo de Schrijver, como mostra uma segunda imersão planar desse grafo ilustrada na figura 5.6, obtida por Williams [2004].

Vamos considerar agora o problema de determinar quais grafos orientados satisfazem a conjectura de Edmonds-Giles para qualquer função capacidade. Chame o grafo orientado do contra-exemplo de Schrijver de $D_{1}$ e os grafos orientados dos contra-exemplos de Cornuéjols e Guenin de $D_{2}$ e $D_{3}$. É fácil ver que, se um grafo orientado $D$ pode ser contraído a uma extensão transitiva de $D_{1}, D_{2}$ ou $D_{3}$, então existe uma função capacidade sobre seus arcos com relação à qual $D$ é um contra-exemplo para a conjectura de Edmonds-Giles: basta atribuir uma capacidade suficientemente grande aos arcos contraídos, as capacidades do contraexemplo apropriado aos arcos de $D_{i}$, e capacidade 0 aos arcos transitivos. CornuÉJols E GUENIN [2002] conjecturaram que também vale a afirmação recíproca:

\section{Conjectura 5.9}

Seja $D=(V, A)$ um grafo orientado. Se existe uma função capacidade $c: A \rightarrow \mathbb{Z}_{\geq 0}$ tal que a capacidade mínima de um corte orientado é estritamente maior que o tamanho máximo de uma família $c$-disjunta de junções, então $D$ pode ser contraído a uma extensão transitiva de $D_{1}, D_{2}$ ou $D_{3}$.

Williams [2004] forneceu diversos contra-exemplos para essa conjectura modificando os grafos orientados $D_{1}, D_{2}$ e $D_{3}$. Poderíamos tentar consertar a asserção da conjectura 5.9 adicionando os novos grafos orientados encontrados por Williams. Porém, isso aumentaria demais o número de subestruturas proibidas na conjectura. Para contornar essa dificuldade, Williams propôs o uso de várias outras operações de redução além da contração de arcos. Com isso, ele modificou a asserção da conjectura 5.9 da seguinte maneira:

\section{Conjectura 5.10}

Seja $D=(V, A)$ um grafo orientado. Se existe uma função capacidade $c: A \rightarrow \mathbb{Z}_{\geq 0}$ tal que a capacidade mínima de um corte orientado é estritamente maior que o tamanho máximo de uma família $c$-disjunta de junções, então $D$ pode ser reduzido a algum grafo orientado de $\mathcal{D}_{1} \cup \mathcal{D}_{2} \cup \mathcal{D}_{3}$.

Nessa afirmação, $\mathcal{D}_{1}:=\left\{D_{1}\right\}$ e, para $i=2,3$, $\mathcal{D}_{i}$ é uma família de oito grafos orientados obtidos a partir de $D_{i}$, e as operações de redução são as introduzidas por Williams. $\mathrm{Na}$ verdade, as operações de redução de Williams se aplicam a pares $(D, c)$, onde $c$ é uma função capacidade sobre os arcos do grafo orientado $D$, e as subestruturas proibidas de $\mathcal{D}_{1} \cup \mathcal{D}_{2} \cup \mathcal{D}_{3}$ também são pares da forma $(D, c)$. Decidimos, porém, omitir esse detalhe técnico.

Williams [2004] estudou propriedades dos contra-exemplos minimais para a conjectura de Edmonds-Giles, onde a minimalidade é com relação às suas operações de redução. Ele provou que, se a capacidade mínima de um corte orientado é 2 , então as capacidades dos arcos são 0 ou 1 e os arcos de capacidade 1 formam pseudocaminhos disjuntos da mesma forma que nos contra-exemplos de Schrijver e Cornuéjols e Guenin, isto é, os vértices internos 
são fontes ou sorvedouros que induzem cortes orientados de capacidade mínima e os arcos de cada pseudocaminho são alternadamente diretos e reversos. Ele mostrou ainda que o número desses pseudocaminhos é pelo menos 3 e que os únicos contra-exemplos minimais com exatamente 3 desses pseudocaminhos são os grafos orientados de $\mathcal{D}_{1} \cup \mathcal{D}_{2} \cup \mathcal{D}_{3}$. Além disso, ele provou que não existe nenhum contra-exemplo minimal com exatamente 4 desses pseudocaminhos.

Ressaltamos que os contra-exemplos em que a capacidade mínima de um corte orientado é 2 parecem ser muito importantes. Cornú́Jols, Guenin e Margot [2000] conjecturaram, no contexto muito mais geral de clutters, que a capacidade mínima de um corte orientado é 2 para todo contra-exemplo minimal para a conjectura de Edmonds-Giles.

WiLliams [2004] conjecturou ainda que todo grafo acíclico que possui uma super-fonte e um super-sorvedouro satisfaz a conjectura de Edmonds-Giles, onde uma super-fonte é uma fonte que acessa todos os sorvedouros e um super-sorvedouro é um sorvedouro acessível a partir de todas as fontes. Note que, se provada, essa afirmação generalizaria o teorema 4.5.1 das junções disjuntas de Feofiloff-Younger e Schrijver.

\subsection{Empacotamento fracionário de junções}

Seja $D=(V, A)$ um grafo orientado. Denote por $\mathcal{J}$ o conjunto das junções de $D$.

A versão capacitada do teorema de Lucchesi-Younger (teorema 3.2) pode ser reformulada em termos de total dual integralidade da seguinte maneira: o sistema de inequações lineares

$$
\begin{array}{ll}
x_{a} \geq 0 & \text { para todo } a \in A, \\
x(\vec{\delta}(S)) \geq 1 & \text { para todo corte orientado } \vec{\delta}(S),
\end{array}
$$

é totalmente dual integral. Pelo teorema 1.7, o poliedro determinado por esse sistema é integral. Como o clutter dos cortes orientados minimais é o bloqueador do clutter das junções minimais, segue da teoria de poliedros bloqueadores e anti-bloqueadores (teorema 1.2) que o poliedro determinado por

$$
\begin{array}{ll}
x_{a} \geq 0 & \text { para todo } a \in A, \\
x(J) \geq 1 & \text { para todo } J \in \mathcal{J},
\end{array}
$$

também é integral. Portanto, é óbvio que os vértices desse poliedro são vetores de incidência de cortes orientados. Assim, pelo teorema 1.3 de dualidade, temos que, para qualquer função capacidade $c: A \rightarrow \mathbb{R}_{\geq 0}$, a capacidade mínima de um corte orientado é igual a

$$
\max \left\{\lambda(\mathcal{J}): \lambda \geq 0, \sum_{J \in \mathcal{J}} \lambda_{J} \chi^{J} \leq c\right\}
$$

Motivados por essa relação, definimos um empacotamento fracionário de junções, ou simplesmente empacotamento fracionário, como um vetor $\lambda$ indexado por $\mathcal{J}$ tal que $\lambda \geq 0$ e $\sum_{J \in \mathcal{J}} \lambda_{J} \chi^{J} \leq c$. Definimos o valor de um empacotamento fracionário $\lambda$ como $\lambda(\mathcal{J})$. Um empacotamento fracionário $\lambda$ é semi-integral se $2 \lambda$ é integral. 
Uma possibilidade de pesquisa para a conjectura de Edmonds-Giles seria descobrir se o sistema (5.2) é totalmente dual semi-integral, ou seja, se para qualquer função capacidade $c: A \rightarrow \mathbb{Z}_{\geq 0}$, existe um empacotamento fracionário de valor máximo que é semi-integral. Se isso fosse provado, teríamos a seguinte relação min-max: a capacidade mínima de um corte orientado é igual à metade do número máximo de junções tais que cada arco $a$ aparece em no máximo $2 c(a)$ dessas junções. Nessa direção, Shepherd E VetTa [2005] provaram o seguinte resultado:

\section{Teorema 5.11}

Seja $D=(V, A)$ um grafo orientado e $c: A \rightarrow \mathbb{Z}_{\geq 0}$ uma função capacidade. Então existe um empacotamento fracionário semi-integral cujo valor é metade da capacidade mínima de um corte orientado.

Demonstração. Seja $k$ a capacidade mínima de um corte orientado. Fixe um vértice $r \in V$. Para cada arco do grafo, crie um arco reverso com capacidade $k$. Chame o grafo orientado resultante de $D^{\prime}$ e a nova função capacidade de $c^{\prime}$. É claro que a capacidade mínima de um $r$-corte em $D^{\prime}$ (com relação a $c^{\prime}$ ) é pelo menos $k$. Pelo corolário 4.1.2, existe uma família $c^{\prime}$-disjunta de $k r$-arborescências, digamos, $\left\{B_{1}^{\prime}, \ldots, B_{k}^{\prime}\right\}$. Tome $B_{i}:=B_{i}^{\prime} \cap A$ para cada $i$ e observe que $\left\{B_{1}, \ldots, B_{k}\right\}$ forma uma família $c$-disjunta. Ademais, para todo corte orientado $\overrightarrow{\delta_{D}}(U)$ de $D$ tal que $r \notin U$, temos que $B_{i} \cap \overrightarrow{\delta_{D}}(U) \neq \varnothing$ para todo $i$.

Aplicando o argumento acima ao grafo $D^{-1}$, obtemos uma família $c$-disjunta $\left\{\tilde{B}_{1}, \ldots, \tilde{B}_{k}\right\}$ satisfazendo a seguinte propriedade: para todo corte orientado $\vec{\delta}_{D}(U)$ tal que $r \in U$, vale que $\tilde{B}_{i} \cap \vec{\delta}_{D}(U) \neq \varnothing$ para todo $i$. Tome $J_{i}:=B_{i} \cup \tilde{B}_{i}$ para $i=1, \ldots, k$. Então $J_{i}$ é uma junção para cada $i$. Para obter um empacotamento fracionário $\lambda$ como prometido pelo enunciado, basta tomar $\lambda_{J}:=1 / 2$ se $J \in\left\{J_{1}, \ldots, J_{k}\right\}$ e $\lambda_{J}:=0$ para as demais junções $J$.

Vamos descrever agora um algoritmo combinatório fortemente polinomial que constrói um empacotamento fracionário de valor máximo. Esse algoritmo é devido a SchriJver [2003, capítulo 57].

Seja $D=(V, A)$ um grafo orientado. Para qualquer função capacidade $c: A \rightarrow \mathbb{R}_{\geq 0}$, denote por $\tau(c)$ a capacidade mínima de um corte orientado, e por $\nu^{*}(c)$ o valor máximo de um empacotamento fracionário, ambos com relação a $c$. Nosso algoritmo recebe uma função capacidade $c: A \rightarrow \mathbb{R}_{\geq 0}$ e encontra um empacotamento fracionário de valor $\nu^{*}(c)$.

A idéia básica do algoritmo é a seguinte. Se $\tau(c)=0$, devolvemos o vetor $\mathbf{0}$. Já se $\tau(c)>0$, tentamos encontrar uma junção $J$ e um real positivo $\lambda_{J}$ tal que

$$
c-\lambda_{J} \chi^{J} \geq 0
$$

$\mathrm{e}$

$$
\tau\left(c-\lambda_{J} \chi^{J}\right)=\tau(c)-\lambda_{J}
$$

Se conseguirmos encontrar tais $J$ e $\lambda_{J}$, podemos aplicar indutivamente o algoritmo sobre $c^{\prime}:=c-\lambda_{J} \chi^{J}$ para obter um empacotamento fracionário $\lambda^{\prime}$ (com relação a $c^{\prime}$ ) de valor $\nu^{*}\left(c^{\prime}\right)=\tau\left(c^{\prime}\right)=\tau(c)-\lambda_{J}$. Assim, $\lambda:=\lambda^{\prime}+\lambda_{J} \chi^{J}$ será um empacotamento fracionário com relação a $c$ de valor $\tau(c)=\nu^{*}(c)$, como queremos. 
Seja $J$ uma junção e $\lambda_{J}$ um real não-negativo. Dizemos que $\lambda_{J}$ é admissível para $J$ se $J$ e $\lambda_{J}$ satisfazem (5.4) e é redutor para $J$ se $J$ e $\lambda_{J}$ satisfazem (5.5). Dizemos que $J$ é admissível se existe um real positivo que é admissível para $J$. Dizemos que $J$ é redutora se existe um real positivo que é redutor para $J$.

O algoritmo é iterativo. A cada iteração construímos uma junção admissível $J$ que será candidata a redutora. Depois calculamos o maior real $\lambda_{J}^{*}$ que é admissível e redutor para $J$. Se $\lambda_{J}^{*}>0$, prosseguimos para a iteração seguinte $\operatorname{com} c-\lambda_{J}^{*} \chi^{J}$ no lugar de $c$. A dificuldade surge quando $\lambda_{J}^{*}=0$. Se isso ocorre, precisamos garantir que a junção construída na iteração seguinte será, de alguma forma, melhor que a junção $J$. Para tanto, vamos manter uma lista $\mathcal{U}$ de subconjuntos de $V$ tal que, a cada iteração, $c(\vec{\delta}(U))=\tau(c)$ para todo $U \in \mathcal{U}$. Vamos usar a lista $\mathcal{U}$ para construir a junção $J$ a cada iteração e, sempre que $J$ não for redutora, vamos aumentar a lista $\mathcal{U}$ para que as junções seguintes sejam "melhores".

Observe que, se $J$ é uma junção, $\lambda_{J}$ é um real e $\vec{\delta}(S)$ é um corte orientado, então $\left(c-\lambda_{J} \chi^{J}\right)(\vec{\delta}(S))=c(\vec{\delta}(S))-\lambda_{J} \vec{d}_{J}(S)$. Logo, a junção $J$ é redutora exatamente se $\vec{d}_{J}(S)=1$ para todo corte orientado $\vec{\delta}(S)$ tal que $c(\vec{\delta}(S))=\tau(c)$. Por essa razão, a junção admissível construída a cada iteração será tal que $\vec{d}_{J}(U)=1$ para todo $U \in \mathcal{U}$.

Prosseguimos a descrição do algoritmo da seguinte forma. Primeiro vamos mostrar como construir uma junção admissível $J$ tal que $\vec{d}_{J}(U)=1$ para todo $U \in \mathcal{U}$. Depois descrevemos como calcular o maior real $\lambda_{J}^{*}$ que é admissível e redutor para $J$. Finalmente, vamos provar que o algoritmo descrito é fortemente polinomial.

Para construir uma junção admissível $J$ tal que $\vec{d}_{J}(U)=1$ para todo $U \in \mathcal{U}$, basta usar o algoritmo de Frank (descrito no capítulo 3) para encontrar uma junção de comprimento mínimo com relação à função comprimento $\ell^{\prime}$ definida da seguinte forma. Comece tomando $\ell:=\sum_{U \in \mathcal{U}} \chi^{\vec{\delta}^{(U)}}$. Agora tome $\ell^{\prime}(a):=\ell(a)$ se $c(a)>0$ e $\ell^{\prime}(a):=\infty$ se $c(a)=0$. Para fins de implementação, podemos substituir $\infty$ por $|\mathcal{U}|+1$. Vamos mostrar que tal junção satisfaz as propriedades desejadas.

Primeiro vamos mostrar que

$\mathcal{U}$ é uma família de núcleos que atinge o máximo no teorema capacitado de Lucchesi-Younger (teorema 3.2), com relação à função comprimento $\ell^{\prime}$.

Basta encontrarmos uma junção $J$ que satisfaz com $\mathcal{U}$ as condições de otimalidade (3.12). Seja $\lambda^{\prime}$ um empacotamento fracionário de valor máximo (com relação a $c$ ). Como $\tau(c)>0$, então $\lambda_{J}^{\prime}>0$ para alguma junção $J$. Pelas condições de folgas complementares do programa linear $(5.3)$ e seu dual (teorema 1.4 ), temos que $\vec{d}_{J}(S)=1$ para todo corte orientado $\vec{\delta}(S)$ de capacidade mínima. Em particular, temos que $\vec{d}_{J}(U)=1$ para todo $U \in \mathcal{U}$. Portanto, a condição (3.12)(iii) é satisfeita. Como $\lambda_{J}^{\prime}>0$, nenhum arco de $J$ tem capacidade nula, de modo que $\ell(a)=\ell^{\prime}(a)$ para todo arco $a \in J$. Note que qualquer arco $a$ entra em exatamente $\ell(a)$ dos núcleos de $\mathcal{U}$. Mas então as condições (3.12)(i) e (3.12)(ii) também são satisfeitas. Isso conclui a prova da afirmação (5.6). 
Seja $J$ uma junção de comprimento mínimo com relação a $\ell^{\prime}$. Pela afirmação (5.6), temos que $J$ e $\mathcal{U}$ satisfazem as condições de otimalidade (3.12). Vamos mostrar que $J$ é admissível e satisfaz $\vec{d}_{J}(U)=1$ para todo $U \in \mathcal{U}$. Esta última propriedade é imediata de (3.12)(iii). Como $|\mathcal{U}|$ é finito, é claro da condição $(3.12)(\mathrm{i})$ que $\ell(a)=\ell^{\prime}(a)$ para todo $a \in J$, de modo que $c(a)>0$ para todo $a \in J$. Portanto, $J$ é admissível.

Agora vamos mostrar como encontrar o maior real $\lambda_{J}^{*}$ que é admissível e redutor para uma junção admissível $J$. Considere a função de $\lambda_{J}$ definida como $f\left(\lambda_{J}\right):=\tau\left(c-\lambda_{J} \chi^{J}\right)$. Note que

$$
f\left(\lambda_{J}\right)=\min \left\{c(\vec{\delta}(S))-\lambda_{J} \vec{d}_{J}(S): \vec{\delta}(S) \text { é um corte orientado }\right\} .
$$

Segue que $f\left(\lambda_{J}\right)$ é uma função côncava e linear por partes. Para encontrar o valor desejado de $\lambda_{J}^{*}$, prosseguimos da seguinte maneira. Começamos tomando $\lambda_{J}^{*}:=\min \{c(a): a \in J\}>0$. Se $f\left(\lambda_{J}^{*}\right)=\tau(c)-\lambda_{J}^{*}$, já cumprimos a nossa missão. Caso contrário, encontramos um corte orientado de capacidade mínima com relação a $c-\lambda_{J}^{*} \chi^{J}$, digamos, $\vec{\delta}(U)$. Note que $\vec{d}_{J}(U)>1$. Agora modificamos $\lambda_{J}^{*}$ da seguinte maneira: o novo valor de $\lambda_{J}^{*}$ é a abscissa do ponto de intersecção das retas $\tau(c)-\lambda_{J}$ e $c(\vec{\delta}(U))-\lambda_{J} \vec{d}_{J}(U)$, ou seja, o novo valor de $\lambda_{J}^{*}$ é

$$
\frac{c(\vec{\delta}(U))-\tau(c)}{\vec{d}_{J}(U)-1} .
$$

Agora repetimos o processo até encontrarmos $\lambda_{J}^{*}$ tal que $f\left(\lambda_{J}^{*}\right)=\tau(c)-\lambda_{J}^{*}$. Chamamos as iterações desse processo de iterações internas para distingui-las das iterações externas, que são as que constroem $J$, executam as iterações internas e atualizam a função capacidade.

Vamos mostrar mais adiante que esse processo de obtenção de $\lambda_{J}^{*}$ é fortemente polinomial, e portanto termina. Ao fim do processo, se o valor inicial de $\lambda_{J}^{*}$ foi modificado alguma vez, adicione à lista $\mathcal{U}$ o conjunto $U$ tal que $\vec{\delta}(U)$ foi o corte orientado usado na última modificação de $\lambda_{J}^{*}$. Note que, com relação a $c-\lambda_{J}^{*} \chi^{J}$, o corte orientado $\vec{\delta}(U)$ tem capacidade mínima, e portanto pode ser adicionado a $\mathcal{U}$.

Agora vamos provar que o algoritmo descrito é fortemente polinomial. Vejamos primeiro que, fixada uma junção $J$, podemos encontrar o valor desejado de $\lambda_{J}^{*}$ após no máximo $|J|$ iterações internas. Suponha que, numa iteração interna e na seguinte, o valor de $\lambda_{J}^{*}$ foi modificado. Chame os objetos $\lambda_{J}^{*}$ e $U$ de $\lambda_{J}$ e $U$ numa iteração e de $\lambda_{J}^{\prime}$ e $U^{\prime}$ na iteração seguinte. Como $\vec{\delta}(U)$ tem capacidade mínima com relação $c-\lambda_{J} \chi^{J}$, então

$$
\left(c-\lambda_{J} \chi^{J}\right)(\vec{\delta}(U)) \leq\left(c-\lambda_{J} \chi^{J}\right)\left(\vec{\delta}\left(U^{\prime}\right)\right) .
$$

Além disso, como na iteração interna seguinte o valor de $\lambda_{J}^{\prime}$ foi modificado usando (5.7), então

$$
\left(c-\lambda_{J}^{\prime} \chi^{J}\right)\left(\vec{\delta}\left(U^{\prime}\right)\right)<\tau(c)-\lambda_{J}^{\prime}=\left(c-\lambda_{J}^{\prime} \chi^{J}\right)(\vec{\delta}(U)) .
$$

Mas então

$$
\lambda_{J}^{\prime}\left(\vec{d}_{J}(U)-\vec{d}_{J}\left(U^{\prime}\right)\right)<c(\vec{\delta}(U))-c\left(\vec{\delta}\left(U^{\prime}\right)\right) \leq \lambda_{J}\left(\vec{d}_{J}(U)-\vec{d}_{J}\left(U^{\prime}\right)\right) .
$$

Como $\tau\left(c-\lambda_{J} \chi^{J}\right)<\tau(c)-\lambda_{J}$, então $\lambda_{J}^{\prime}<\lambda_{J}$, e portanto $\vec{d}_{J}\left(U^{\prime}\right)<\vec{d}_{J}(U)$. Segue que o número máximo de iterações internas para o cálculo de $\lambda_{J}^{*}$ é $|J|$. 
Antes de limitarmos o número de iterações externas, precisamos de algumas definições. Uma coleção $\mathcal{R}$ de subconjuntos de $V$ é um reticulado se é fechada sob união e intersecção, isto é, se $U \cap W, U \cup W \in \mathcal{R}$ sempre que $U, W \in \mathcal{R}$. Não é difícil ver que todo reticulado não-vazio é completamente determinado pelo seu conjunto minimal $L$, pelo seu conjunto maximal $M$ e pela pré-ordem $\preceq$ definida a seguir (uma pré-ordem é uma relação reflexiva e transitiva). Dados $u, v \in V$, escrevemos $u \preceq v$ se todo conjunto de $\mathcal{R}$ que contém $v$ também contém $u$. Não é difícil ver que $S \subseteq V$ está em $\mathcal{R}$ se, e somente se, $L \subseteq S \subseteq M$ e $S$ é fechada sob $\preceq$, ou seja, para todo $v \in S$ e para todo $u \preceq v$, vale que $u \in S$.

Seja $\mathcal{R}^{\prime}$ outro reticulado. Seja $L^{\prime}$ o conjunto minimal de $\mathcal{R}^{\prime}, M^{\prime}$ o conjunto maximal de $\mathcal{R}^{\prime}$ e $\preceq^{\prime}$ a pré-ordem associada a $\mathcal{R}^{\prime}$. Lembramos que uma relação sobre $V$ é um subconjunto do produto cartesiano $V \times V$, de modo que o tamanho de uma pré-ordem está bem definido. Se $\mathcal{R} \subsetneq \mathcal{R}^{\prime}$, então ou $L^{\prime} \subsetneq L$, ou $M^{\prime} \supsetneq M$, ou então o tamanho da pré-ordem $\preceq$ ' é estritamente maior que o da pré-ordem $\preceq$. Portanto, se temos um reticulado $\mathcal{R}$ e queremos aumentar a coleção $\mathcal{R}$, sempre preservando a propriedade de que $\mathcal{R}$ é um reticulado, podemos aumentar $\mathcal{R}$ no máximo $|V||V \times V|=|V|^{3}$ vezes.

Lembramos que uma coleção $\mathcal{C}$ de subconjuntos de $V$ é fechada sob cruzamentos se $U \cap W, U \cup W \in \mathcal{C}$ sempre que $U, W \in \mathcal{C}$ se cruzam. Seja $\mathcal{S}$ uma coleção de subconjuntos de $V$. A coleção fechada sob cruzamentos gerada por $\mathcal{S}$ é a intersecção de todas as coleções fechadas sob cruzamentos e que contêm $\mathcal{S}$.

Voltemos à análise do algoritmo. Precisamos limitar o número de iterações externas. Considere uma iteração externa qualquer. Ocorre exatamente um dentre os seguintes casos: ou o valor encontrado pelas iterações internas foi $\lambda_{J}^{*}=\min \{c(a): a \in J\}$, ou então o valor inicial de $\lambda_{J}^{*}$ foi modificado pelo menos uma vez, de modo que um certo conjunto $U$ será adicionado à lista $\mathcal{U}$. Se $\lambda_{J}^{*}=\min \{c(a): a \in J\}$, então na iteração seguinte, o número de arcos com capacidade positiva é estritamente menor. Logo, esse caso ocorre em no máximo $|A|$ das iterações externas.

Suponha então que um conjunto $U$ será adicionado à lista $\mathcal{U}$. Vimos que tal $U$ satisfaz $\vec{d}_{J}(U)>1$. Seja $\mathcal{U}^{\times}$a coleção fechada sob cruzamentos gerada por $\mathcal{U}$. Pela submodularidade da função $\vec{d}_{J}(S)$, temos que $\vec{d}_{J}(U)=1$ para todo $U \in \mathcal{U}^{\times}$. Logo, $U \notin \mathcal{U}^{\times}$. Observe que, para cada par de vértices distintos $x, y \in V$, a coleção $\mathcal{U}_{x, \bar{y}}^{\times}:=\left\{U \in \mathcal{U}^{\times}: x \in U, y \notin U\right\}$ é um reticulado. Logo, ao adicionarmos $U$ à lista $\mathcal{U}$, pelo menos um dos reticulados $\mathcal{U}_{x, \bar{y}}^{\times}$aumenta. Pelas observações anteriores, cada reticulado não-vazio pode aumentar no máximo $|V|^{3}$ vezes. Logo, no máximo $|V|^{2}|V|^{3}+|V|^{2}$ das iterações externas são desse tipo (a parcela $|V|^{2}$ leva em conta os reticulados que passam de vazios a não-vazios).

Concluímos que o número total de iterações (externas e internas) é limitado por um polinômio em $|V|$ e $|A|$. Como todos os demais passos podem ser executados em tempo fortemente polinomial, segue que o nosso algoritmo é fortemente polinomial.

Não é difícil combinar esse algoritmo com o teorema 4.5.1 das junções disjuntas de Feofiloff-Younger e Schrijver para obter um algoritmo semi-fortemente polinomial (ou seja, fortemente polinomial exceto por certas operações de arredondamento) para a versão capacitada do problema do empacotamento máximo de junções em grafos fonte-sorvedouro-conexos. Veja SchriJver [2003, capítulos 51, 56 e 57]. 


\subsection{O teorema de Lee e Williams}

LEE E WiLliams [2006] provaram recentemente que a conjectura planar de Edmonds-Giles vale para todo grafo orientado planar livre de $K_{5}-e$ como menor, usando uma caracterização de tais grafos obtida por WAGNer [1960]. Lembramos que $K_{5}-e$ é o grafo obtido do $K_{5}$ após a remoção de uma aresta. Segue por dualidade no plano que a conjectura de Edmonds-Giles vale para todo grafo orientado planar livre do envelope como menor, onde o envelope, também conhecido como prisma ou prisma triangular, é o grafo ilustrado na figura 5.7. Nesta seção, vamos provar esse resultado.

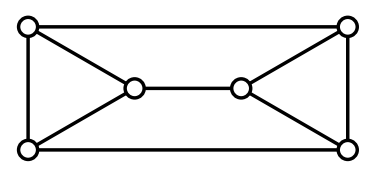

Figura 5.7: O envelope. O dual planar é $K_{5}-e$.

Começamos com um teorema de decomposição de WAGner [1960]. Uma roda é um grafo da forma $C \times K_{1}$ para algum circuito $C$ de comprimento pelo menos 3 .

\section{Teorema 5.12}

Os únicos grafos simples que são planares, 3-conexos e não têm $K_{5}-e$ como menor são as rodas e o envelope.

Não provaremos este teorema neste texto. Notamos, porém, que ele pode ser facilmente provado usando o seguinte teorema de TUTTE [1961b]:

\section{Teorema 5.13}

Um grafo simples $G$ é 3 -conexo se, e somente se, existem grafos simples $G_{0}, \ldots, G_{k}$ tais que $G_{0}=K_{4}, G_{k}=G$ e, para $i=0, \ldots, k-1$, o grafo $G_{i+1}$ possui uma aresta $x y$ tal que $G_{i}=G_{i+1} / x y$ e ambos $x$ e $y$ têm grau pelo menos 3 em $G_{i+1}$.

Uma prova curta desse resultado foi obtida por Thomassen [1980] e também pode ser vista no livro de Diestel [2005].

Para provar o teorema 5.12 de Wagner usando o teorema 5.13, proceda da seguinte forma. Seja $G$ um grafo 3-conexo planar e livre de $K_{5}-e$ como menor. Sejam $G_{0}, \ldots, G_{k}$ grafos como no teorema de Tutte. Então cada $G_{i}$ é planar e livre de $K_{5}-e$ como menor. Note ainda que $G_{0}$ é uma roda. Agora uma simples análise de casos mostra que, se $G_{i}$ é uma roda, então $G_{i+1}$ ou é uma roda, ou é o envelope, ou então contém $K_{5}-e$ como menor. Ademais, se $G_{i}$ é o envelope e $i<k$, então $G_{i+1}$ contém $K_{5}-e$ como menor.

Antes de provarmos o teorema de Lee e Williams, precisamos introduzir um pouco de terminologia e notação. Precisamos também provar alguns lemas.

Seja $D$ um grafo orientado e $w: A_{D} \rightarrow \mathbb{Z}_{\geq 0}$ uma função peso. Denotamos o peso mínimo de um circuito de $D$ por $\tau(D, w)$ e o tamanho máximo de uma família $w$-disjunta de quebracircuitos de $D$ por $\nu(D, w)$. 


\section{Lema $5.14 \alpha$}

Seja $D=(V, A)$ um grafo orientado e $w: A \rightarrow \mathbb{Z}_{\geq 0}$ uma função peso. Suponha que existem vértices $x, y \in V$ tais que $D^{\prime}:=D-x-y$ não é fracamente conexo. Seja $U$ o conjunto de vértices de um dos componentes fracamente conexos de $D^{\prime}$. Tome $D_{1}^{\prime}:=D[U \cup\{x, y\}]$ e $D_{2}^{\prime}:=D-U-A[\{x, y\}]$. Para $i=1,2$, seja $w_{i}^{\prime}$ a função $w$ restrita a $A\left(D_{i}^{\prime}\right)$ e $\alpha_{i}$ o peso mínimo de um caminho de $x$ para $y$ em $D_{i}^{\prime}$ com relação a $w_{i}^{\prime}$. Tome $\alpha:=\min \left\{\tau(D, w), \alpha_{1}, \alpha_{2}\right\}$. Sejam $a:=x y$ e $b:=y x$ arcos novos. Para $i=1,2$, tome $D_{i}:=D_{i}^{\prime}+a+b$ e defina uma função peso $w_{i}$ sobre $A\left(D_{i}\right)$ a partir de $w_{i}^{\prime}$ atribuindo peso $\alpha$ ao arco $a$ e $\tau(D, w)-\alpha$ ao arco $b$. Se $\tau\left(D_{i}, w_{i}\right)=\nu\left(D_{i}, w_{i}\right)$ para $i=1,2$, então $\tau(D, w)=\nu(D, w)$.

Demonstração. Abrevie $\tau:=\tau(D, w)$ e $\nu:=\nu(D, w)$. Abrevie também $\tau_{i}:=\tau\left(D_{i}, w_{i}\right)$ e $\nu_{i}:=\nu\left(D_{i}, w_{i}\right)$ para $i=1,2$. Começamos mostrando que

$$
\tau_{i}=\tau, \text { para } i=1,2
$$

É claro que $\tau_{i} \leq \tau$ devido aos arcos novos $a$ e $b$. Suponha que existe um circuito $C$ em $D_{i}$ com $w_{i}(C)<\tau$. É claro então que $C$ passa por $a$ ou por $b$. Suponha primeiro que $C$ passa por $b$. Então $C-b$ é um caminho de $x$ para $y$ em $D_{i}^{\prime}$ e $w_{i}^{\prime}(C-b)<\alpha \leq \alpha_{i}$, o que contradiz a escolha de $\alpha_{i}$. Concluímos que $C$ deve passar por $a$. Então $C-a$ é um caminho de $y$ para $x$ em $D_{i}^{\prime}$ com $w_{i}^{\prime}(C-a)<\tau-\alpha_{i}$. Seja $P_{x y}$ um caminho de $x$ para $y$ em $D_{i}^{\prime}$ tal que $w_{i}^{\prime}\left(P_{x y}\right)=\alpha_{i}$. Então $(C-a) \cup P_{x y}$ contém um circuito $C^{\prime}$ tal que $w\left(C^{\prime}\right)<\tau-\alpha_{i}+\alpha_{i}=\tau$. Essa contradição conclui a prova de (5.8).

Para $i=1,2$, como $\tau=\tau_{i}$ e $\tau_{i}=\nu_{i}$ por hipótese, então existem $\tau$ quebra-circuitos de $D_{i}$ que são $w_{i}$-disjuntos, digamos, $Q_{1}^{i}, \ldots, Q_{\tau}^{i}$. Como o circuito formado pelos arcos $a$ e $b$ tem peso $\tau$, podemos supor que $Q_{j}^{i} \cap\{a, b\}=\{a\}$ para todo $j \in\{1, \ldots, \alpha\}$ e que $Q_{j}^{i} \cap\{a, b\}=\{b\}$ para todo $j \in\{\alpha+1, \ldots, \tau\}$.

Tome $Q_{j}:=\left(Q_{j}^{1} \cup Q_{j}^{2}\right) \backslash\{a, b\}$ para $j=1, \ldots, \tau$. Afirmamos que

$$
Q_{j} \text { é um quebra-circuitos de } D \text { para todo } j=1, \ldots, \tau \text {. }
$$

De fato, seja $C$ um circuito de $D$. Se $C$ é um circuito de $D_{1}^{\prime}$ ou de $D_{2}^{\prime}$, então é evidente que $Q_{j} \cap C \neq \varnothing$. Suponha então que $C$ passa pelos vértices $x$ e $y$ e que $C=P_{x y} \cup P_{y x}$, onde $P_{x y}$ é um caminho de $x$ para $y$ e $P_{y x}$ é um caminho de $y$ para $x$. Vamos supor que $P_{x y}$ é um caminho em $D_{1}^{\prime}$ e que $P_{y x}$ é um caminho em $D_{2}^{\prime}$. O outro caso é análogo.

Suponha que $j \in\{1, \ldots, \alpha\}$. Como $P_{x y}+b$ é um circuito em $D_{1}$ e $b \notin Q_{j}^{1}$, temos que $Q_{j} \cap P_{x y} \neq \varnothing$. Suponha então que $j \in\{\alpha+1, \ldots, \tau\}$. Como $P_{y x}+a$ é um circuito em $D_{2}$ e $a \notin Q_{j}^{2}$, temos que $Q_{j} \cap P_{y x} \neq \varnothing$. Em ambos os casos, $Q_{j} \cap C \neq \varnothing$. Isso conclui a prova da afirmação (5.9).

Como $\left\{A\left(D_{1}^{\prime}\right), A\left(D_{2}^{\prime}\right)\right\}$ é uma partição de $A$, então $Q_{1}, \ldots, Q_{\tau}$ são obviamente $w$-disjuntos, como queríamos. 
Com esse lema, já podemos derivar o seguinte resultado de LeE E WAKABAYASHI [2001]:

\section{Teorema 5.14}

Seja $D=(V, A)$ um grafo orientado e $w: A \rightarrow \mathbb{Z}_{\geq 0}$ uma função peso. Se $D$ é livre de $K_{4}$ como menor, então o peso mínimo de um circuito é igual ao tamanho máximo de uma família $w$-disjunta de quebra-circuitos.

Demonstração. Seja $D$ um contra-exemplo com o menor número de vértices.

Uma simples análise de casos mostra que a afirmação do teorema é válida se $D$ possui no máximo 3 vértices. Logo, podemos supor que $D$ tem pelo menos 4 vértices.

Seja $G$ o grafo subjacente a $D$. Pela minimalidade de $\left|V_{D}\right|$, é óbvio que $G$ é 2-conexo. Se existem $x, y \in V$ distintos tais que $G-x-y$ é desconexo, então $\tau(D, w)=\nu(D, w)$ pela minimalidade de $\left|V_{D}\right|$ e pelo lema $5.14 \alpha$. Concluímos que $G$ é 3-conexo. Mas é fácil ver que todo grafo 3 -conexo possui $K_{4}$ como menor. Portanto, $D$ não é livre de $K_{4}$ como menor. Essa contradição conclui a prova.

Precisamos introduzir agora mais notação e terminologia.

Seguindo as mesmas idéias da prova do teorema 5.3, dizemos que um quebra-circuitos $Q$ de $D$ é admissível (para $w)$ se $\chi^{Q} \leq w$ e é adequado $($ em $(D, w))$ se $\tau\left(D, w-\chi^{Q}\right) \geq$ $\tau(D, w)-1$. Por conveniência, se $Q$ é um quebra-circuitos admissível para $w$ e adequado em $(D, w)$, dizemos que $Q$ é admissível e adequado para $(D, w)$.

Observe que, se $Q_{1}, \ldots, Q_{\tau}$ é uma família $w$-disjunta de quebra-circuitos de $D$ tal que $\tau=\tau(D, w)$, então $Q_{j}$ é um quebra-circuitos admissível e adequado para $(D, w)$, para todo $j$.

Vamos usar ainda uma operação que altera os pesos nos arcos de um corte. Seja $U \subsetneq V$ um subconjunto não-vazio. $\mathrm{O}$ deslocamento de $w$ para dentro de $U$ é a operação que transforma $w$ na função peso

$$
\varrho(w, U):=w+\chi^{\vec{\delta}(U)}-\chi^{\overleftarrow{\delta}(U)}
$$

Suponha que $w^{\prime}:=\varrho(w, U) \geq 0$. É evidente que $w(C)=w^{\prime}(C)$ para todo circuito $C$ de $D$. Logo,

$$
\tau(D, w)=\tau(D, \varrho(w, U)) .
$$

Além disso, é claro que

$$
\begin{aligned}
& \text { um quebra-circuitos é adequado em }(D, w) \text { se, e somente se, } \\
& \text { é adequado em }(D, \varrho(w, U)) \text {. }
\end{aligned}
$$

Será conveniente realizarmos sucessivos deslocamentos para dentro de $U$ até que algum arco que sai de $U$ obtenha peso 0 . Para cada $i \geq 0$, denote

$$
\varrho^{i}(w, U):= \begin{cases}w, & \text { se } i=0, \\ \varrho\left(\varrho^{i-1}(w, U), U\right), & \text { caso contrário. }\end{cases}
$$

Denote ainda

$$
\overleftarrow{w}_{\min }(U):= \begin{cases}\min \{w(a): a \in \overleftarrow{\delta}(U)\}, & \text { se } \overleftarrow{\delta}(U) \neq \varnothing \\ \tau(D, w), & \text { caso contrário. }\end{cases}
$$

Finalmente, denote $\varrho^{*}(w, U):=\varrho^{i^{*}}(w, U)$, onde $i^{*}:=\overleftarrow{w}_{\min }(U)$. 


\section{Lema $5.15 \alpha$ (Lema fraco da remoção)}

Seja $D=(V, A)$ um grafo orientado e $w: A \rightarrow \mathbb{Z}_{\geq 0}$ uma função peso. Suponha que existe um arco $a \in A \operatorname{com} w(a) \geq \tau(D, w)$. Seja $D^{\prime}:=D-a$ e $w^{\prime}$ a função peso $w$ restrita aos arcos de $D^{\prime}$. Se $\tau\left(D^{\prime}, w^{\prime}\right)=\nu\left(D^{\prime}, w^{\prime}\right)$, então $\tau(D, w)=\nu(D, w)$.

Demonstração. Abrevie $\tau:=\tau(D, w)$. É evidente que $\tau\left(D^{\prime}, w^{\prime}\right) \geq \tau$. Sejam $Q_{1}, \ldots, Q_{\tau}$ quebra-circuitos $w^{\prime}$-disjuntos de $D^{\prime}$. Então $Q_{1} \cup\{a\}, \ldots, Q_{\tau} \cup\{a\}$ são quebra-circuitos $w$-disjuntos de $D$.

\section{Lema $5.15 \beta$ (Lema forte da remoção)}

Seja $D=(V, A)$ um grafo orientado e $w: A \rightarrow \mathbb{Z}_{\geq 0}$ uma função peso. Suponha que existe um subconjunto não-vazio $U \subsetneq V$ bem como um arco $a \in \vec{\delta}(U)$ tais que $w(a)+\overleftarrow{w}_{\min }(U) \geq \tau(D, w)$ e $\overleftarrow{w}_{\min }(U) \cdot \vec{d}_{A_{0}}(U)<\tau(D, w)$, onde $A_{0}:=\{a \in A: w(a)=0\}$. Seja $D^{\prime}:=D-a$ e $w^{\prime}$ a função peso $\varrho^{*}(w, U)$ restrita aos arcos de $D^{\prime}$. Se $\tau\left(D^{\prime}, w^{\prime}\right)=\nu\left(D^{\prime}, w^{\prime}\right)$, então existe um quebra-circuitos admissível e adequado para $(D, w)$.

Demonstração. Abrevie $\tau:=\tau(D, w)$ e $w^{\prime \prime}:=\varrho^{*}(w, U)$. Suponha que $\tau\left(D^{\prime}, w^{\prime}\right)=\nu\left(D^{\prime}, w^{\prime}\right)$. Pela equação (5.10), temos que $\tau=\tau\left(D, w^{\prime \prime}\right)$. Como $w(a)+\overleftarrow{w}_{\min }(U) \geq \tau$, então $w^{\prime \prime}(a) \geq \tau$. Logo, pelo lema fraco da remoção, existem $\tau$ quebra-circuitos $w^{\prime \prime}$-disjuntos de $D$, digamos, $Q_{1}, \ldots, Q_{\tau}$. Pela afirmação (5.11), como $Q_{j}$ é adequado em $\left(D, w^{\prime \prime}\right)$, então $Q_{j}$ é adequado em $(D, w)$. Resta encontrarmos, dentre os quebra-circuitos $Q_{1}, \ldots, Q_{\tau}$, um quebra-circuitos que é admissível para $w$. Observe que um quebra-circuitos $Q_{j}$ pode não ser admissível para $w$, já que $Q_{j}$ pode conter um arco $b \in \vec{\delta}(U)$ tal que $w(b)=0$ e $w^{\prime \prime}(b)>0$. Porém, como

$$
\sum\left\{w^{\prime \prime}(b): b \in \vec{\delta}_{A_{0}}(U)\right\}=\overleftarrow{w}_{\min }(U) \cdot \vec{d}_{A_{0}}(U)<\tau
$$

então pelo menos um dos $\tau$ quebra-circuitos $Q_{1}, \ldots, Q_{\tau}$ não tem nenhum arco de $\vec{\delta}_{A_{0}}(U)$, e portanto é admissível.

\section{Lema $5.15 \gamma$}

Seja $D$ um grafo orientado. Seja $C$ um pseudocircuito de $D$ que não é um circuito. Se $Q$ é um quebra-circuitos minimal que inclui todos os arcos diretos de $C$, então $Q$ inclui pelo menos um arco reverso de $C$.

Demonstração. Como $Q$ é minimal, para todo arco $a \in Q$, existe um circuito $C_{a}$ tal que $C_{a} \cap Q=\{a\}$. Denote o conjunto dos arcos direto de $C$ por $C^{\rightarrow}$ e tome $C^{\leftarrow}:=C \backslash C^{\rightarrow}$. É fácil ver que, no grafo $C^{*}:=\left(C^{\leftarrow} \cup \bigcup\left\{C_{a}: a \in C^{\rightarrow}\right\}\right) \backslash C^{\rightarrow}$, todo vértice é ponta inicial de algum arco e todo vértice é ponta final de algum arco. Mas então existe um circuito em $C^{*}$ que só pode intersectar $Q$ em $C^{\leftarrow}$.

Podemos finalmente provar o teorema de LEE E WiLliams [2006]:

\section{Teorema 5.15 (Teorema de Lee e Williams)}

Seja $D=(V, A)$ um grafo orientado e $w: A \rightarrow \mathbb{Z}_{\geq 0}$ uma função peso. Suponha que $D$ é planar e livre de $K_{5}-e$ como menor. Então o peso mínimo de um circuito é igual ao tamanho máximo de uma família $w$-disjunta de quebra-circuitos de $D$. 
Demonstração. Dentre todos os contra-exemplos $(D, w)$, escolha os que minimizam $\tau(D, w)$. Dentre esses, escolha os que minimizam $\left|V_{D}\right|$. Finalmente, dentre esses, escolha um que minimiza $\left|A_{D}\right|$. Seja $(D, w)$ o contra-exemplo escolhido.

Começamos observando algumas propriedades de $D$ e $w$. Abrevie $\tau:=\tau(D, w)$.

Observe que

não existe nenhum quebra-circuitos admissível e adequado para $(D, w)$.

De fato, suponha que $Q$ é um quebra-circuitos admissível e adequado para $(D, w)$. Tome a função peso $w^{\prime}:=w-\chi^{Q}$. Então $\tau\left(D, w^{\prime}\right)=\tau-1$. Pela minimalidade de $\tau(D, w)$, temos que $\tau\left(D, w^{\prime}\right)=\nu\left(D, w^{\prime}\right)$. Assim, combinando $\nu\left(D, w^{\prime}\right)=\tau-1$ quebra-circuitos $w^{\prime}$-disjuntos com $Q$, obtemos $\tau$ quebra-circuitos $w$-disjuntos em $D$, o que contradiz o fato de que $(D, w)$ é um contra-exemplo para o teorema.

Chame um arco de crítico se ele está em algum circuito de peso $\tau$. Podemos supor que

todo arco com peso positivo é crítico.

De fato, suponha que um arco $a \operatorname{com} w(a)>0$ não está em nenhum circuito de peso $\tau$. Diminua o peso de $a$ de modo que $\tau(D, w)$ seja preservado e que ou a passe a estar em algum circuito de peso mínimo ou então seu novo peso seja 0 . Por exemplo, se $a=x y$, tome $w^{\prime}(a):=\max \{0, \tau-\alpha\}$, onde $\alpha$ denota o peso mínimo de um caminho de $y$ para $x$. Repita isso para cada arco de $D$. Chame de $w^{\prime}$ a função peso obtida ao fim desse procedimento e note que $w^{\prime} \leq w$ e $\tau\left(D, w^{\prime}\right)=\tau$. Então, com relação à função peso $w^{\prime}$, cada arco de $D$ com peso positivo é crítico. Ademais, $\left(D, w^{\prime}\right)$ é um contra-exemplo pois, se $Q_{1}, \ldots, Q_{\tau}$ são quebra-circuitos $w^{\prime}$-disjuntos, então eles também são $w$-disjuntos. Isso conclui a prova de que podemos supor a validade de (5.13).

É fácil ver ainda que, pela escolha do contra-exemplo,

$$
D \text { não possui laços nem arcos paralelos. }
$$

Além disso, é óbvio que

todo arco está em algum circuito; em particular, $D$ não tem cortes orientados.

Finalmente, notamos que,

se dois $\operatorname{arcos} a$ e $b$ formam um circuito, então $w(a), w(b)>0$.

De fato, se $w(a)=0$, então $w(b) \geq \tau$. Pela minimalidade de $\left|A_{D}\right|$ e pelo lema fraco da remoção, existem $\tau$ quebra-circuitos $w$-disjuntos em $D$, contradizendo o fato de que $(D, w)$ é um contra-exemplo.

Seja $G$ o grafo simples subjacente a $D$. Como na prova do teorema 5.14, $G$ é 3 -conexo. Pelo teorema 5.12 de Wagner, temos que $G$ é uma roda ou um envelope. Em ambos os casos, $G$ possui um vértice de grau 3 . Seja $u$ um tal vértice e sejam $x, y, z$ seus vizinhos em $G$. Pela afirmação (5.15), temos que $\vec{\delta}(u) \neq \varnothing$ e $\overleftarrow{\delta}(u) \neq \varnothing$. Assim, pela afirmação (5.14), as únicas configurações possíveis para os arcos incidentes em $u$ são as que estão listadas na figura 5.8. 


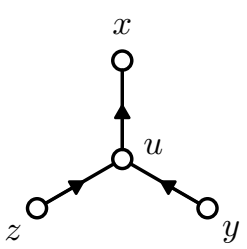

(1)

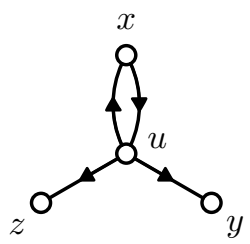

(5)

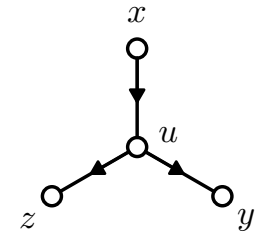

$(2)$

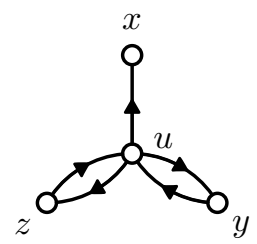

(6)

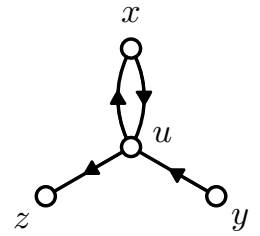

(3)

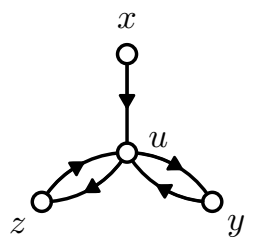

(7)

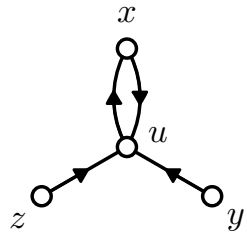

$(4)$

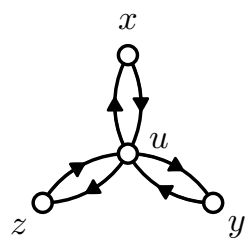

(8)

Figura 5.8: As configurações possíveis para os arcos incidentes em $u$.

Vamos mostrar que nenhuma das configurações de (3) a (8) é possível. Começamos eliminando as configurações (6) a (8). Em todas essas configurações, existe no máximo um arco que não forma um circuito de comprimento 2. Pela afirmação (5.16), esse é o único arco que pode ter peso 0 . Assim, escolhendo apropriadamente um conjunto $U$ como $U:=\{u\}$ ou $U:=V \backslash\{u\}$, teremos que $\vec{d}_{A_{0}}(U)=0$, de modo que podemos aplicar o lema forte da remoção sobre $U$ e algum arco apropriado de $\vec{\delta}(U)$ para obter um quebra-circuitos admissível e adequado em $(D, w)$. Mas isso contradiz a afirmação (5.12).

Vamos eliminar agora as configurações (4) e (5). Por simetria, basta eliminarmos a configuração (4). Chame de $C$ o circuito formado pelos $\operatorname{arcos} u x$ e $x u$. Note que $C$ é o único circuito de $D$ que passa por $x u$. Tome $D^{\prime}:=D-x u$ e seja $w^{\prime}$ a restrição de $w$ aos $\operatorname{arcos}$ de $D^{\prime}$. Vamos mostrar que

$$
\tau=\tau\left(D^{\prime}, w^{\prime}\right)
$$

É evidente que $\tau\left(D^{\prime}, w^{\prime}\right) \geq \tau$. Pela afirmação (5.16), temos que $w(x u)>0$. Assim, obtemos da afirmação (5.13) que $\tau=w(u x)+w(x u)$. Pela afirmação (5.15), o arco zu está em algum circuito de $D$, digamos $C^{\prime}$, e tal circuito certamente passa pelo arco $u x$. Observe que pelo menos um arco de $C^{\prime}-u x$ tem peso positivo, pois caso contrário, teríamos que $w\left(C^{\prime}\right)=w(u x)<w(u x)+w(x u)=\tau$. Seja $a$ um tal arco. É evidente que nenhum circuito de $D$ que passa por $a$ também passa por $x u$. Agora a equação (5.17) segue da afirmação (5.13).

Pela minimalidade de $\left|A_{D}\right|$, temos que $\tau\left(D^{\prime}, w^{\prime}\right)=\nu\left(D^{\prime}, w^{\prime}\right)$. Portanto, existem $\tau$ quebracircuitos $w^{\prime}$-disjuntos em $D^{\prime}$. Agora adicione o arco $x u$ a cada um dos quebra-circuitos que não contém $u x$ para obter $\tau$ quebra-circuitos $w$-disjuntos de $D$. Mas então $\tau=\nu(D, w)$, contradizendo o fato de que $(D, w)$ é um contra-exemplo. 
Resta eliminarmos a configuração (3). Se $w(y u) \geq w(x u)$ ou $w(u z) \geq w(u x)$, então podemos eliminar a configuração (3) com um argumento semelhante ao que usamos para eliminar as configurações (6)-(8). Suponha então que

$$
w(y u)<w(x u) \quad \text { e } \quad w(u z)<w(u x) .
$$

Remova o vértice $u$ e adicione arcos entre os vértices $x, y$ e $z$ como na figura 5.9, atribuindo os pesos $w(y u)+w(u x)$ ao arco $y x, w(y u)+w(u z)$ ao arco $y z$ e $w(x u)+w(u z)$ ao arco $x z$. Chame o grafo resultante de $D^{\prime}$ e a função peso resultante de $w^{\prime}$.
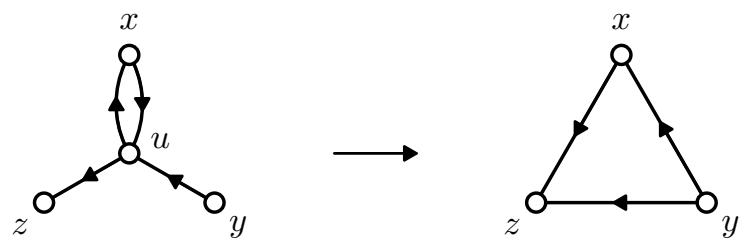

Figura 5.9: Modificando a configuração 3.

Note que, com essa alteração, todos os circuitos de $D$ têm um circuito correspondente em $D^{\prime}$ e com o mesmo peso, exceto pelo circuito formado pelos arcos $u x$ e $x u$, que obviamente não aparece em $D^{\prime}$. Afirmamos que

$$
\tau=w(u x)+w(x u) .
$$

Pois suponha que $\tau<w(u x)+w(x u)$. Por (5.18), sabemos que $w(u x)>0$ e $w(x u)>0$. Pela afirmação (5.13), o arco ux está num circuito $C_{u x}$ de peso mínimo e o arco $x u$ está num circuito $C_{x u}$ de peso mínimo. Mas então o grafo orientado $D$ possui um circuito $C$ tal que $C \subseteq\left(C_{x u}-x u\right) \cup\left(C_{u x}-u x\right)$ e com peso $w(C) \leq \tau-w(x u)+\tau-w(u x)<\tau$. Essa contradição conclui a prova de (5.19).

Usando um argumento semelhante ao usado na prova de (5.17), podemos mostrar que o circuito formado pelos $\operatorname{arcos} u x$ e $x u$ não é o único circuito de peso mínimo de $D$, de modo que $\tau\left(D^{\prime}, w^{\prime}\right)=\tau$. Pela minimalidade de $\left|V_{D}\right|$, temos que $\tau\left(D^{\prime}, w^{\prime}\right)=\nu\left(D^{\prime}, w^{\prime}\right)$. Sejam $Q_{1}^{\prime}, \ldots, Q_{\tau}^{\prime}$ quebra-circuitos $w^{\prime}$-disjuntos de $D^{\prime}$. Vamos mostrar que pelo menos um desses quebra-circuitos pode ser modificado para um quebra-circuitos admissível e adequado para $(D, w)$.

Seja $Q_{i}^{\prime}$ um dos quebra-circuitos de $D^{\prime}$ e $C^{\prime}$ o pseudocircuito de $D^{\prime}$ formado pelos arcos $y x$, $y z$ e $x z$. Pelo lema $5.15 \gamma$, as únicas possibilidades para o conjunto $Q_{i}^{\prime} \cap C^{\prime}$ são as ilustradas na figura 5.10, onde os arcos de $Q_{i}^{\prime} \cap C^{\prime}$ são exatamente os arcos pontilhados.

Pelas relações (5.18) e (5.19), sabemos que o arco $y z$ aparece em no máximo $w^{\prime}(y z)=$ $w(y u)+w(u z)<w(u x)+w(x u)=\tau$ dos quebra-circuitos $Q_{1}^{\prime}, \ldots, Q_{\tau}^{\prime}$. Logo, pelo menos um dos quebra-circuitos $Q_{i}^{\prime}$ é do tipo (a), (b) ou (c) na figura 5.10. Se $Q_{i}^{\prime}$ é do tipo (a) ou (b), então é fácil ver que $Q_{i}^{\prime} \cup\{u x\}$ é um quebra-circuitos admissível e adequado para $(D, w)$. Já se $Q_{i}^{\prime}$ é do tipo (c), então é fácil ver que $Q_{i}^{\prime} \cup\{x u\}$ é um quebra-circuitos admissível e adequado para $(D, w)$. Em ambos os casos, obtemos um quebra-circuitos admissível e adequado para $(D, w)$, contradizendo a afirmação (5.12). 


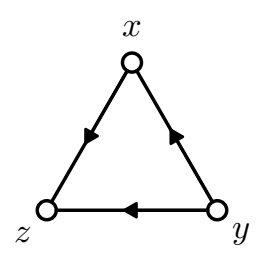

(a)

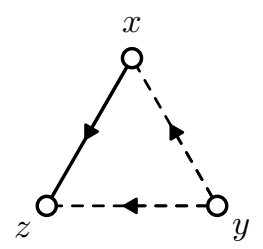

(d)

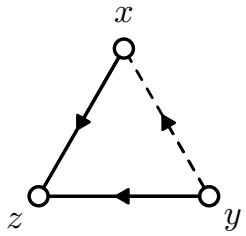

(b)

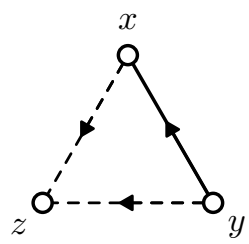

(e)

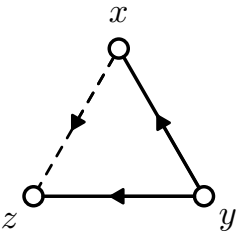

(c)

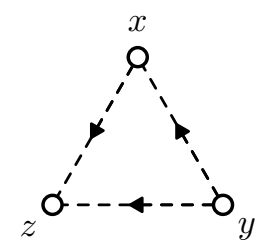

(f)

Figura 5.10: As possibilidades para $Q_{i}^{\prime} \cap C^{\prime}$.

Logo, as únicas configurações possíveis para os arcos incidentes em vértices de grau $3 \mathrm{em} G$ são as configurações (1) e (2) da figura 5.8. Como o envelope é cúbico e na roda existe no máximo um vértice de grau diferente de 3 , segue que o grafo orientado $D$ não tem circuitos de comprimento 2.

Vamos arrematar a prova usando o teorema das junções disjuntas de Feofiloff-Younger e Schrijver. Notamos, entretanto, que não precisamos de um resultado tão poderoso: LEE [2006] observou que, com um pouco mais de trabalho, podemos terminar esta prova sem apelarmos para esse teorema.

Seja $D^{*}$ o dual planar de $D$. Suponha que $G$ é o envelope. Então o dual planar de $G$ é $K_{5}-e$ e, pela afirmação (5.15), o grafo orientado $D^{*}$ é uma orientação acíclica de $K_{5}-e$. É fácil ver que qualquer orientação acíclica de $K_{5}-e$ é fonte-sorvedouro-conexo. De fato, se um dos 3 vértices de grau 4 de $K_{5}-e$ é uma fonte ou um sorvedouro em $D^{*}$, é claro que $D^{*}$ é fonte-sorvedouro-conexo. Caso contrário, um dos vértices de grau 3 é uma fonte e é fácil ver que a $D^{*}$ é fonte-sorvedouro-conexo. Logo, pelo teorema das junções disjuntas de Feofiloff-Younger e Schrijver e por dualidade no plano, existem $\tau$ quebra-circuitos $w$-disjuntos de $D$, uma contradição.

Suponha então que $G$ é uma roda. Então o dual planar de $G$ também é uma roda. É fácil ver que qualquer orientação acíclica de uma roda é fonte-sorvedouro-conexa. Assim, obtemos uma contradição exatamente como no parágrafo anterior.

Concluímos que não existe nenhum contra-exemplo para o teorema.

\section{Corolário 5.15.1}

Seja $D=(V, A)$ um grafo orientado planar e $c: A \rightarrow \mathbb{Z}_{\geq 0}$ uma função capacidade. Se $D$ é livre do envelope como menor, então a capacidade mínima de um corte orientado é igual ao tamanho máximo de uma família $c$-disjunta de junções.

Demonstração. Imediato do teorema 5.15 de Lee e Williams via dualidade no plano. 


\section{Resultados relacionados}

Barahona, Fonlupt E Mahjoub [1994] mostraram que a mesma relação min-max do teorema 5.5 planar de Lucchesi-Younger vale para todo grafo orientado livre de $K_{3,3}$ como menor. Eles se basearam num teorema de WAGNER [1937b] sobre a decomposição de grafos livres de $K_{3,3}$ como menor em grafo planares e cópias do $K_{5}$. Seymour [1996] mostrou que a relação min-max também vale para todo grafo orientado euleriano cujo grafo subjacente é linklessly embeddable, isto é, que pode ser imerso em $\mathbb{R}^{3}$ evitando que quaisquer dois circuitos disjuntos nos vértices estejam ligados da mesma forma que duas argolas de uma corrente. Seymour se baseou na caracterização de tais grafos por menores proibidos obtida por Robertson, Seymour e Thomas [1995]. 



\section{Capítulo 6}

\section{$T$-junções e emparelhamentos}

A teoria de emparelhamentos é muito rica e elegante e permeia as áreas de otimização combinatória e teoria dos grafos. O problema do emparelhamento máximo, por exemplo, é um dos modelos bem-resolvidos mais fundamentais da otimização combinatória. O teorema de emparelhamentos de König, que resolve esse problema em grafos bipartidos, foi uma das primeiras relações min-max descobertas. Posteriormente, as contribuições pioneiras de Tutte e Edmonds resolveram o problema em grafos não-bipartidos. Os artigos seminais de Edmonds publicados em 1965 merecem destaque especial, pois abriram áreas que antes eram inacessíveis pelos métodos clássicos baseados em fluxos e unimodularidade total.

Diversas extensões de emparelhamentos foram propostas. Uma delas envolve o conceito de $T$-junção e está intimamente relacionado com o problema chinês do carteiro: dado um grafo com custos nas arestas, encontre um passeio que percorre cada aresta pelo menos uma vez e que tem o menor custo possível. Esse conceito também está surpreendentemente relacionado com o problema do corte máximo e de multifluxos em grafos planares, bem como outros problemas importantes em otimização combinatória.

Diversas relações min-max para o tamanho mínimo de uma $T$-junção já foram provadas. A maioria delas envolve o empacotamento de $T$-cortes. Frank e Szigeti observaram que um teorema de Frank, Tardos e Sebő implica todas essas relações min-max de forma relativamente simples e que esse próprio teorema pode ser provado facilmente usando uma técnica de Sebő.

Dentre as consequiências desse teorema de Frank, Tardos e Sebő está uma caracterização de Seymour dos grafos que satisfazem uma certa relação min-max envolvendo o tamanho mínimo de uma $T$-junção. Essa caracterização é um caso especial de um difícil teorema de Seymour sobre matróides binários com a propriedade fluxo-máximo corte-mínimo.

Neste capítulo, vamos apresentar o teorema de Frank, Tardos e Sebő e derivar dele diversas relações min-max para o tamanho mínimo de uma $T$-junção. Vamos provar também alguns resultados clássicos sobre emparelhamentos.

O restante do capítulo está organizado da seguinte maneira: na seção 6.1, introduzimos os conceitos de $T$-junção e $T$-corte e provamos o teorema de Frank, Tardos e Sebő; na seção 6.2, derivamos diversas relações min-max clássicas para emparelhamentos; na seção 6.3, derivamos várias relações min-max para o tamanho mínimo de uma $T$-junção; finalmente, na seção 6.4, apresentamos uma prova curta para a caracterização de Seymour. 


\subsection{O teorema de Frank, Tardos e Sebő}

Seja $G=(V, E)$ um grafo e $T \subseteq V$. Dizemos que $J \subseteq E$ é uma $T$-junção se $T$ é o conjunto dos vértices de grau ímpar do grafo $(V, J)$.

Por exemplo, um subconjunto de arestas é uma $\varnothing$-junção se, e somente se, é uma união de circuitos disjuntos nas arestas. Se $r, s \in V$ são distintos, então um subconjunto de arestas é uma $\{r, s\}$-junção minimal se, e somente se, é um caminho que liga $r$ a $s$.

Chame um conjunto $T \subseteq V$ de compatível se $G$ possui uma $T$-junção. É evidente que $T \subseteq V$ é compatível se, e somente se, $|T \cap K|$ é par para cada componente $K$ de $G$.

Sejam $T$ e $T^{\prime}$ conjuntos compatíveis. Observe que,

se $J$ é uma $T$-junção e $J^{\prime}$ é uma $T^{\prime}$-junção, então $J \triangle J^{\prime}$ é uma $\left(T \triangle T^{\prime}\right)$-junção.

De fato, temos que

$$
\begin{aligned}
g_{J \triangle J^{\prime}}(v) & \equiv g_{J \triangle J^{\prime}}(v)+2 g_{J \cap J^{\prime}}(v) & & (\bmod 2) \\
& \equiv g_{J}(v)+g_{J^{\prime}}(v) & & (\bmod 2)
\end{aligned}
$$

ou seja, $g_{J \triangle J^{\prime}}(v)$ é ímpar se, e somente se, precisamente um dentre $g_{J}(v)$ e $g_{J^{\prime}}(v)$ é ímpar.

Usando essas propriedades, é fácil ver que

toda $T$-junção é uma união de circuitos e $|T| / 2$ caminhos disjuntos nas arestas, onde os caminhos ligam pares disjuntos de vértices de $T$.

Uma $T$-junção é mínima se tem tamanho mínimo dentre todas as $T$-junções. O seguinte fato foi observado por GUAN [1960]:

\section{Lema 6.1 $\alpha$ (Lema de Guan)}

Seja $G$ um grafo e $T$ um conjunto compatível. Então uma $T$-junção $J$ é mínima se, e somente se, $|C \cap J| \leq|C \backslash J|$ para todo circuito $C$ de $G$.

Demonstração. Seja $J$ uma $T$-junção mínima e $C$ um circuito de $G$. Pela afirmação (6.1), o conjunto de arestas $J^{\prime}:=J \triangle C$ é uma $T$-junção. Como $|J| \leq\left|J^{\prime}\right|$, então é óbvio que $|C \cap J| \leq|C \backslash J|$.

Seja $J$ uma $T$-junção e suponha que existe uma $T$-junção $J^{\prime}$ satisfazendo $\left|J^{\prime}\right|<|J|$. Pela afirmação (6.1), o conjunto $J \triangle J^{\prime}$ é uma $\varnothing$-junção, ou seja, é uma união de circuitos disjuntos nas arestas. É claro que, para pelo menos um desses circuitos, digamos, $C$, vale que $|C \cap J|>|C \backslash J|$, pois caso contrário teríamos $\left|J^{\prime}\right| \geq|J|$.

Uma função $w: E \rightarrow\{+1,-1\}$ é conservativa se $w(C) \geq 0$ para todo circuito $C$ de $G$. Dado um conjunto compatível $T$ e uma $T$-junção $J$, defina uma função peso $w^{J}: E \rightarrow \mathbb{Z}$ da seguinte maneira: para cada $e \in E$, tome

$$
w^{J}(e):= \begin{cases}-1, & \text { se } e \in J \\ +1, & \text { caso contrário. }\end{cases}
$$


É óbvio que a seguinte afirmação é equivalente ao lema $6.1 \alpha$ de Guan:

$$
\text { uma } T \text {-junção } J \text { é mínima se, e somente se, } w^{J} \text { é conservativa. }
$$

Dizemos que $S \subseteq V$ é $T$-par se $|S \cap T|$ é par e é $T$-ímpar se $|S \cap T|$ é ímpar. Dizemos que $F \subseteq E$ é um $T$-corte se $F=\delta(S)$ para algum subconjunto $T$-ímpar $S$. Note que

$$
d_{J}(S) \text { é ímpar se, e somente se, } S \text { é } T \text {-ímpar. }
$$

Isso segue imediatamente da seguinte equação:

$$
\sum_{v \in S} g_{J}(v)=d_{J}(S)+2|E[S] \cap J|
$$

Em particular, toda $T$-junção intersecta todo $T$-corte, e portanto o tamanho mínimo de uma $T$-junção nunca é menor que o número máximo de $T$-cortes disjuntos.

Seja $H$ um subgrafo de $G$. Dizemos que $H$ é $T$-par se $V_{H}$ é $T$-par e é $T$-ímpar se $V_{H}$ é $T$-ímpar. Denote por $q_{T}(H)$ o número de componentes $T$-ímpares de $H$.

Estamos prontos para provar o teorema de Frank, TARdos e Sebö [1984]. Seguimos a prova apresentada no artigo de FrAnk e SzIGEti [1994], que usa uma técnica de SEBö [1987].

\section{Teorema 6.1 (Teorema de Frank, Tardos e Sebő)}

Seja $G=(V, E)$ um grafo $(U, W)$-bipartido e $T$ um conjunto compatível. Então o tamanho mínimo de uma $T$-junção é igual a

$$
\max \left\{\sum_{S \in \mathcal{U}} q_{T}(G-S)\right\}
$$

onde o máximo é tomado sobre todas as partições $\mathcal{U}$ de $U$.

Demonstração. Primeiro mostramos que $\min \geq \max$. Seja $J$ uma $T$-junção e $\mathcal{U}$ uma partição de $U$. Como $G$ é $(U, W)$-bipartido, temos que

$$
\begin{aligned}
|J| & =\sum_{S \in \mathcal{U}} \sum\left\{d_{J}(K): K \text { é um componente de } G-S\right\} \\
& \geq \sum_{S \in \mathcal{U}} \sum\{1: K \text { é um componente } T \text {-ímpar de } G-S\} \\
& =\sum_{S \in \mathcal{U}} q_{T}(G-S)
\end{aligned}
$$

já que, se $K$ é um componente $T$-ímpar de $G-S$, então $\delta(K)$ é um $T$-corte, e portanto $d_{J}(K) \geq 1$. 
Vamos mostrar agora que existem uma $T$-junção $J$ e uma partição $\mathcal{U}$ de $U$ que satisfazem a inequação (6.6) com igualdade. Para tanto, basta mostrarmos que

existem uma $T$-junção $J$ e uma partição $\mathcal{U}$ de $U$ tais que, para cada parte $S \in \mathcal{U}$ e cada componente $K$ de $G-S$, existe no máximo uma aresta de $J$ com uma ponta em $S$ e outra em $K$.

Observe que isso faz com que a única inequação de (6.6) valha com igualdade.

Seja $J$ uma $T$-junção mínima. Pela afirmação (6.3), a função peso $w^{J}$ é conservativa. Chame uma aresta de negativa se seu peso é negativo. Para provarmos (6.7), é suficiente provarmos que,

se $w$ é uma função conservativa, então existe uma partição $\mathcal{U}$ de $U$ tal que, para cada parte $S \in \mathcal{U}$ e cada componente $K$ de $G-S$, existe no máximo uma aresta negativa com uma ponta em $S$ e outra em $K$.

Seja $J$ o conjunto das arestas negativas. Nossa prova é por indução em $|J|$. Se $J=\varnothing$, basta tomarmos $\mathcal{U}:=\{U\}$. Suponha então que $J \neq \varnothing$.

Nossa estratégia será encontrar um vértice apropriado $s$ que incida numa única aresta negativa. Depois vamos contrair as arestas do corte $\delta(s)$ para obter um novo grafo bipartido. Se tomarmos cuidado na escolha de $s$, a função $w$ restrita às arestas do grafo resultante ainda será conservativa e poderemos usar indução. No fim, incluímos o conjunto $\{s\}$ na partição encontrada de acordo com a conveniência.

Seja $r$ um vértice incidente em alguma aresta negativa. Dentre todos os caminhos $P$ de $G$ que começam em $r$, escolha os que minimizam $w(P)$. Dentre esses, escolha um que tem o menor número de arestas. Seja $P$ o caminho escolhido. Seja $s$ a outra extremidade de $P$ e $x s$ a aresta de $P$ incidente em $s$.

Pela escolha de $r$, é evidente que $w(P)<0$. Para cada vértice $v$ de $P$ distinto de $s$, denote por $P_{v s}$ o subcaminho de $P$ que vai de $v$ até $s$. Como $P$ tem o menor número possível de arestas, é claro que

$$
w\left(P_{v s}\right)<0 \text { para todo vértice } v \text { de } P \text { distinto de } s \text {. }
$$

Afirmamos que

$$
x s \text { é a única aresta negativa incidente em } s \text {. }
$$

De fato, suponha que sy é outra aresta negativa. Se $P$ passa por $y$, então por (6.9) o circuito $P_{y s}+s y$ tem peso negativo, uma contradição. Logo, $P$ não passa por $y$. Mas então $P+s y$ é um caminho de peso menor que o de $P$, contradizendo a escolha de $P$. Isso conclui a prova de (6.10). 
Tome $G^{\prime}:=G / \delta(s)$. Seja $w^{\prime}: E\left(G^{\prime}\right) \rightarrow\{+1,-1\}$ a função peso correspondente a $w$. Queremos mostrar que

$$
w^{\prime} \text { é conservativa. }
$$

Para tanto, basta mostrarmos que,

em $G-s$, não existe nenhum caminho de peso negativo ligando vizinhos de $s$.

Pois suponha que $Q$ é um caminho em $G-s$ com extremidades $y, z \in N(s)$ e com peso negativo. É claro que $y$ e $z$ são distintos de $x$, pois caso contrário $Q+z s+s y$ seria um circuito de peso negativo.

Para cada vértice $v$ de $Q$, denote por $Q_{y v}$ o subcaminho de $Q$ que liga $y$ a $v$ e por $Q_{v z}$ o subcaminho de $Q$ que liga $v$ a $z$. Note que, como $w(Q)<0$, então, para cada vértice $v$ de $Q$, temos que $w\left(Q_{y v}\right)<0$ ou $w\left(Q_{v z}\right)<0$.

Suponha que $P$ e $Q$ possuem um vértice em comum. Dentre todos os vértices $v$ comuns a $P$ e $Q$, escolha o que minimiza o comprimento de $P_{v s}$. Podemos supor que $w\left(Q_{y v}\right)<0$. Mas então $\left(Q_{y v} \cup P_{v s}\right)+s y$ é um circuito de peso negativo, por (6.9). Isso é uma contradição. Logo, $P$ e $Q$ não têm vértices em comum.

Como $G$ é bipartido e as extremidades de $Q$ estão na mesma classe de cor, então $Q$ tem comprimento par. Logo, $w(Q) \leq-2$. Mas então $(P+s y) \cup Q$ é um caminho que começa em $r$ e que tem peso menor que $w(P)$, contradizendo a escolha de $P$. Essa contradição conclui a prova de (6.12), e portanto a de (6.11).

Estamos prontos para aplicar a hipótese de indução. Seja $s^{\prime} \in V\left(G^{\prime}\right)$ o vértice contraído. Suponha que $s \in U$. Tome $U^{\prime}:=U \backslash\{s\}$ e $W^{\prime}:=\left(W \backslash N_{G}(s)\right) \cup\left\{s^{\prime}\right\}$. Observe que $G^{\prime}$ é $\left(U^{\prime}, W^{\prime}\right)$-bipartido. Pela hipótese de indução, existe uma partição $\mathcal{U}^{\prime}$ de $U^{\prime}$ tal que, para cada parte $S^{\prime} \in \mathcal{U}^{\prime}$ e cada componente $K^{\prime}$ de $G^{\prime}-S^{\prime}$, existe no máximo uma aresta negativa com uma ponta em $S^{\prime}$ e outra em $K^{\prime}$. Então $\mathcal{U}^{\prime} \cup\{\{s\}\}$ satisfaz (6.8).

Suponha agora que $s \in W$. Tome $U^{\prime}:=\left(U \backslash N_{G}(s)\right) \cup\left\{s^{\prime}\right\}$ e $W^{\prime}:=W \backslash\{s\}$. Note que $G^{\prime}$ é $\left(U^{\prime}, W^{\prime}\right)$-bipartido. Pela hipótese de indução, existe uma partição $\mathcal{U}^{\prime}$ de $U^{\prime}$ tal que, para cada parte $S^{\prime} \in \mathcal{U}^{\prime}$ e cada componente $K^{\prime}$ de $G^{\prime}-S^{\prime}$, existe no máximo uma aresta negativa com uma ponta em $S^{\prime}$ e outra em $K^{\prime}$. Seja $S^{\prime}$ a parte de $\mathcal{U}^{\prime}$ que contém $s^{\prime}$. Tome $S:=\left(S^{\prime} \backslash\left\{s^{\prime}\right\}\right) \cup N_{G}(s)$ e $\mathcal{U}:=\left(\mathcal{U}^{\prime} \backslash\left\{S^{\prime}\right\}\right) \cup\{S\}$ e note que $\mathcal{U}$ satisfaz (6.8).

\section{Corolário 6.1.1}

Seja $G=(V, E)$ um grafo e $T$ um conjunto compatível. Então o tamanho mínimo de uma $T$-junção é igual a

$$
\max \left\{\frac{1}{2} \sum_{S \in \mathcal{V}} q_{T}(G-S)\right\}
$$

onde o máximo é tomado sobre todas as partições $\mathcal{V}$ de $V$.

Demonstração. Subdivida cada aresta de $G$, isto é, substitua cada aresta $e=x y \in E$ por um caminho $x w_{e} y$, onde $w_{e}$ é um vértice novo para cada $e \in E$. Seja $G^{\prime}$ o grafo resultante. Tome $W:=\left\{w_{e}: e \in E\right\}$. Agora o corolário segue do teorema 6.1 de Frank, Tardos e Sebö aplicado ao grafo $(V, W)$-bipartido $G^{\prime}$, tomando partições de $V$. 


\subsection{Emparelhamentos}

Nesta seção, vamos derivar alguns resultados clássicos sobre emparelhamentos. Como algumas das provas são bem conhecidas, não vamos nos deter em muitos detalhes.

Seja $G=(V, E)$ um grafo. Lembramos que $M \subseteq E$ é um emparelhamento se cada vértice de $G$ é ponta de no máximo uma aresta de $M$. Se $u v \in M$, dizemos que $u$ está emparelhado com $v$. Dizemos que $M$ satura um vértice $v$ se $v$ é ponta de alguma aresta de $M$ e que $M$ satura $S \subseteq V$ se $M$ satura cada vértice de $S$. Um emparelhamento é perfeito se satura $V$. Note que um emparelhamento perfeito é uma $V$-junção mínima.

Começamos com a famosa caracterização de TutTe [1947] de grafos que admitem um emparelhamento perfeito:

Corolário 6.1.2 (Teorema de emparelhamentos perfeitos de Tutte)

Um grafo $G=(V, E)$ possui um emparelhamento perfeito se, e somente se,

$$
q_{V}(G-S) \leq|S|
$$

para todo $S \subseteq V$.

Demonstração. É fácil ver que a condição (6.14) é necessária, já que cada componente ímpar de $G-S$ possui pelo menos um vértice emparelhado com um vértice de $S$.

Suponha que $G$ não possui um emparelhamento perfeito. Se $V$ não é compatível, então a condição (6.14) é violada por $S:=\varnothing$. Suponha então que $V$ é compatível. Então o tamanho mínimo de uma $V$-junção é estritamente maior que $|V| / 2$. Pelo corolário 6.1.1, existe uma partição $\mathcal{V}$ de $V$ tal que

$$
\frac{1}{2} \sum_{S \in \mathcal{V}} q_{V}(G-S)>|V| / 2=\frac{1}{2} \sum_{S \in \mathcal{V}}|S|
$$

Então existe uma parte $S$ de $\mathcal{V}$ tal que $q_{V}(G-S)>|S|$, como queríamos.

Derivamos agora a fórmula de Tutte-Berge, uma relação min-max obtida por BERGE [1958] que caracteriza o tamanho máximo de um emparelhamento:

Corolário 6.1.3 (Fórmula de Tutte-Berge)

Num grafo $G=(V, E)$ o tamanho máximo de um emparelhamento é igual a

$$
\min _{S \subseteq V}\left\{\frac{1}{2}\left(|V|+|S|-q_{V}(G-S)\right)\right\}
$$

Demonstração. Assim como na prova do teorema de emparelhamentos perfeitos de Tutte, é fácil ver que o máximo nunca excede o mínimo. Seja $k:=\max \left\{q_{V}(G-S)-|S|: S \subseteq V\right\}$. Seja $X$ um conjunto de $k$ vértices novos. É fácil verificar que o grafo $G^{\prime}:=G \times K_{X}$ satisfaz a condição (6.14). Pelo teorema 6.1.2 de emparelhamentos perfeitos de Tutte, o grafo $G^{\prime}$ possui um emparelhamento perfeito $M^{\prime}$. Agora $M:=M^{\prime} \cap E$ é um emparelhamento em $G$ de tamanho (6.15), como queríamos. 
Passemos agora para grafos bipartidos. Começamos com o teorema do casamento de HALL [1935]:

Corolário 6.1.4 (Teorema do casamento de Hall)

Um grafo $(U, W)$-bipartido possui um emparelhamento que satura $U$ se, e somente se,

$$
|N(S)| \geq|S|
$$

para todo $S \subseteq U$.

Demonstração. Claramente a condição (6.16) é necessária. Para ver que ela é suficiente, considere um grafo $G$ que satisfaz (6.16). Se $|V|$ é ímpar, acrescente um vértice novo a $W$ e chame o conjunto resultante de $W^{\prime}$; se $|V|$ é par, tome $W^{\prime}:=W$. Não é difícil ver que o grafo $G^{\prime}:=G \cup K_{W^{\prime}}$ satisfaz a condição (6.14). Logo, pelo teorema 6.1.2 de emparelhamentos perfeitos de Tutte, o grafo $G^{\prime}$ possui um emparelhamento perfeito $M^{\prime}$. É claro que $M:=$ $M^{\prime} \cap E$ é um emparelhamento em $G$ que satura $U$, como queríamos.

Provamos a seguir o teorema de emparelhamentos de KöNIG [1931].

Seja $G=(V, E)$ um grafo. Dizemos que $K \subseteq V$ é uma cobertura por vértices se $G-K$ não possui arestas.

\section{Corolário 6.1.5 (Teorema de emparelhamentos de König)}

Num grafo bipartido, o tamanho mínimo de uma cobertura por vértices é igual ao tamanho máximo de um emparelhamento.

Demonstração. Seja $K$ uma cobertura por vértices de tamanho mínimo. Abrevie $U_{K}:=K \cap U$ e $W_{K}:=K \cap W$. O grafo $G_{U}:=G\left[U_{K} \cup W^{\prime}\right]$, onde $W^{\prime}:=W \backslash W_{K}$, é $\left(U_{K}, W^{\prime}\right)$-bipartido e é fácil ver que ele satisfaz a condição (6.16), pois caso contrário poderíamos encontrar uma cobertura por vértices de tamanho menor que $|K|$. Logo, pelo teorema 6.1.4 do casamento de Hall, o grafo $G_{U}$ possui um emparelhamento $M_{U}$ que satura $U_{K}$. Analogamente, o grafo subgrafo de $G$ induzido por $W_{K} \cup\left(U \backslash U_{K}\right)$ possui um emparelhamento $M_{W}$ que satura $W_{K}$. É claro que $M_{U} \cup M_{W}$ é um emparelhamento em $G$ de tamanho $|K|$, como queríamos.

O último corolário desta seção foi provado por KőNIG [1916]. Uma coloração de arestas de um grafo $G$ é uma partição de $E_{G}$ em emparelhamentos.

\section{Corolário 6.1.6 (Teorema de coloração de arestas de König)}

Num grafo bipartido, o tamanho mínimo de uma coloração de arestas é igual ao grau máximo.

Demonstração. Seja $G$ um grafo bipartido. Tome $\Delta:=\Delta(G)$. É fácil ver que existe um supergrafo $G^{\prime}$ de $G$ que é $\Delta$-regular. Também é fácil ver que todo grafo bipartido regular satisfaz a condição (6.16). Logo, pelo teorema 6.1.4 do casamento de Hall, o grafo $G^{\prime}$ possui um emparelhamento perfeito $M^{\prime}$. É claro que $M:=M^{\prime} \cap E$ é um emparelhamento em $G$ que satura todos os vértices de grau $\Delta$. Mas então $\Delta(G-M)=\Delta-1$ e o teorema segue por indução.

Discutimos aspectos algorítmicos de emparelhamentos nas notas no final do capítulo. 


\subsection{T-junções, $T$-cortes e $T$-fronteiras}

Nesta seção, veremos algumas relações min-max para o tamanho mínimo de uma $T$-junção. Começamos com um resultado de SeYmour [1981]:

\section{Corolário 6.1.7}

Num grafo bipartido, se $T$ é um conjunto compatível, então o tamanho mínimo de uma $T$-junção é igual ao número máximo de $T$-cortes disjuntos.

Demonstração. Imediata do teorema 6.1 de Frank, Tardos e Sebő, tomando o conjunto de $T$-cortes $\{\delta(K): K$ é um componente $T$-ímpar de $G-S$ para algum $S \in \mathcal{U}\}$.

Essa relação min-max em geral não vale para grafos não-bipartidos. Note, por exemplo, que ela não vale se tomarmos $G:=K_{4}$ e $T:=V_{G}$.

Seja $G=(V, E)$ um grafo e $T$ um conjunto compatível. Uma família de $T$-cortes é bidisjunta se cada aresta de $G$ está em no máximo 2 desses $T$-cortes. O valor de uma família bidisjunta de $T$-cortes é a metade do tamanho dessa família.

O seguinte resultado foi provado por Lovász [1975]:

\section{Corolário 6.1.8}

Num grafo, se $T$ é um conjunto compatível, então o tamanho mínimo de uma $T$-junção é igual ao valor máximo de uma família bidisjunta de $T$-cortes.

Demonstração. Imediato do corolário 6.1.7, subdividindo cada aresta.

Seja $G=(V, E)$ um grafo conexo e $T$ um conjunto compatível. Uma partição $\mathcal{P}$ de $V$ é uma $T$-partição se, para toda parte $S$ de $\mathcal{P}$, o subgrafo induzido $G[S]$ é conexo e $S$ é $T$-ímpar. Se $\mathcal{P}$ é uma $T$-partição, então o conjunto de $\operatorname{arestas} \delta_{G}(\mathcal{P})$ é uma $T$-fronteira. Lembramos que $\delta_{G}(\mathcal{P})$ denota o conjunto das arestas de $G$ que têm suas pontas em partes diferentes de $\mathcal{P}$.

Seja $\mathcal{P}$ uma $T$-partição. É claro que $|\mathcal{P}|$ é par. Definimos o valor da $T$-partição $\mathcal{P}$ como $|\mathcal{P}| / 2$ e o valor da $T$-fronteira $\delta(\mathcal{P})$ como o valor de $\mathcal{P}$. Observe que isso está bem definido: se $F \subseteq E$ é uma $T$-fronteira, então existe uma única $T$-partição $\mathcal{P}$ de $V$ tal que $F=\delta(\mathcal{P})$, a saber, a partição $\mathcal{P}=\{K: K$ é um componente de $G-F\}$.

Observe que $\delta(S)$ é um $T$-corte para cada parte $S$ da $T$-partição $\mathcal{P}$. Logo, se $J$ é uma $T$-junção, então $|J \cap \delta(\mathcal{P})|$ é pelo menos o valor da $T$-fronteira $\delta(\mathcal{P})$. O valor de uma família disjunta de $T$-fronteiras é a soma dos valores das $T$-fronteiras da família. É claro que o valor de qualquer família disjunta de $T$-fronteiras é um limitante inferior para o tamanho mínimo de uma $T$-junção.

Vamos derivar agora uma relação min-max provada por SEBő [1988]. Seguimos a prova de Frank e Szigeti [1994]. Lembre-se que, na prova do corolário 6.1.1, subdividimos cada aresta do grafo original e aplicamos o teorema 6.1 de Frank, Tardos e Sebő tomando partições do conjunto original de vértices. Desta vez, vamos tomar partições do conjunto de vértices resultantes das subdivisões. 


\section{Corolário 6.1.9}

Num grafo conexo, se $T$ é um conjunto compatível, então o tamanho mínimo de uma $T$-junção é igual ao valor máximo de uma família disjunta de $T$-fronteiras.

Demonstração. Subdivida cada aresta do grafo $G$, isto é, substitua cada aresta $e=x y \in E$ por um caminho $x u_{e} y$, onde $u_{e}$ é um vértice novo para cada aresta $e \in E$. Chame o grafo resultante de $G^{\prime}$. Tome $U:=\left\{u_{e}: e \in E\right\}$ e note que $G^{\prime}$ é $(U, V)$-bipartido.

Pelo teorema 6.1 de Frank, Tardos e Sebő, existem uma $T$-junção $J^{\prime} \subseteq E\left(G^{\prime}\right)$ e uma partição $\mathcal{U}$ de $U$ tais que

$$
\left|J^{\prime}\right|=\sum_{S \in \mathcal{U}} q_{T}\left(G^{\prime}-S\right)
$$

Escolha uma tal partição $\mathcal{U}$ que maximiza $|\mathcal{U}|$.

Seja $S \in \mathcal{U}$ tal que $q_{T}(S)>0$. Vamos mostrar que

$$
E_{G}(S):=\left\{e \in E: u_{e} \in S\right\} \text { é uma } T \text {-fronteira em } G \text { de valor } q_{T}\left(G^{\prime}-S\right) / 2 .
$$

Basta mostrarmos que $\mathcal{P}:=\left\{K^{\prime} \cap V: K\right.$ é um componente de $\left.G^{\prime}-S\right\}$ é uma $T$-partição e que $E_{G}(S)=\delta_{G}(\mathcal{P})$.

Seja $K \in \mathcal{P}$ e $K^{\prime}$ o componente de $G^{\prime}-S$ tal que $K=K^{\prime} \cap V$. Como $K^{\prime}$ é um componente de $G^{\prime}-S$, é claro que $G[K]$ é conexo. Suponha que $K$ é $T$-par. Então $K^{\prime}$ também é $T$-par. Observe agora que $G^{\prime}-S$ tem no máximo $|S|+1$ componentes. Como $q_{T}\left(G^{\prime}-S\right)>0$, então $G^{\prime}-S$ possui pelo menos 2 componentes $T$-ímpares. Logo, $|S| \geq 2$. Seja $e \in E_{G}(S)$ uma aresta com uma única ponta em $K$. Então $\mathcal{U}^{\prime}:=(\mathcal{U} \backslash\{S\}) \cup\left\{S \backslash\left\{u_{e}\right\},\left\{u_{e}\right\}\right\}$ satisfaz (6.17) e $\left|\mathcal{U}^{\prime}\right|>|\mathcal{U}|$, contradizendo a escolha de $\mathcal{U}$. Concluímos que $\mathcal{P}$ é uma $T$-partição.

É evidente que $\delta_{G}(\mathcal{P}) \subseteq E_{G}(S)$. Suponha que $\delta_{G}(\mathcal{P}) \nsupseteq E_{G}(S)$. Então existe uma aresta $e \in E$ com as duas pontas em algum componente $K$ tal que $u_{e} \in S$. Como $q_{T}\left(G^{\prime}-S\right)>0$, é evidente que $|S| \geq 2$. Então novamente $\mathcal{U}^{\prime}:=(\mathcal{U} \backslash\{S\}) \cup\left\{S \backslash\left\{u_{e}\right\},\left\{u_{e}\right\}\right\}$ satisfaz (6.17) e $\left|\mathcal{U}^{\prime}\right|>|\mathcal{U}|$, contradizendo a escolha de $\mathcal{U}$. Segue que $\delta_{G}(\mathcal{P})=E_{G}(S)$ e isso conclui a prova de $(6.18)$.

Concluímos que $\left\{E_{G}(S): S \in \mathcal{U}\right.$ e $\left.q_{T}\left(G^{\prime}-S\right)>0\right\}$ é uma família disjunta de $T$-fronteiras de valor $\left|J^{\prime}\right| / 2$. Isso conclui a prova, pois $J^{\prime}$ corresponde de maneira óbvia a uma $T$-junção $J$ de $G$ de tamanho $\left|J^{\prime}\right| / 2$.

As notas do final do capítulo contêm algumas referências sobre algoritmos para $T$-junções.

\subsection{Um teorema de Seymour}

Nesta seção, vamos provar um caso especial de um difícil teorema de SEYmour [1977] sobre matróides binários com a propriedade fluxo-máximo corte-mínimo. Esse caso especial caracteriza os pares $(G, T)$ formados por um grafo $G$ e por um conjunto compatível $T$ tais que o tamanho mínimo de uma $T$-junção é igual ao número máximo de $T$-cortes disjuntos. SEBŐ [1988] notou que essa caracterização segue facilmente do corolário 6.1.9 combinado com um certo lema sobre grafos bicríticos. Vamos provar a caracterização usando essa estratégia. Novamente seguimos Frank E SzIGETi [1994]. 
Dizemos que um grafo $G$ é bicrítico se $G-u-v$ tem um emparelhamento perfeito para cada $u, v \in V$ distintos. Pelo teorema 6.1.2 de emparelhamentos perfeitos de Tutte,

um grafo $G$ é bicrítico se, e somente se, $q_{V}(G-S) \leq|S|-2$ para todo subconjunto $S \subseteq V$ tal que $|S| \geq 2$.

Seja $G=(V, E)$ um grafo conexo, $T$ um conjunto compatível e $\delta(\mathcal{P})$ uma $T$-fronteira. O grafo de fronteiras de $\delta(\mathcal{P})$ é o grafo obtido de $G$ através da contração de cada parte de $\mathcal{P}$. Uma $T$-fronteira é bicrítica se seu grafo de fronteiras é bicrítico.

\section{Lema $6.2 \alpha$}

Seja $G=(V, E)$ um grafo conexo e $T$ um conjunto compatível. Se uma família disjunta de $T$-fronteiras de valor máximo tem tamanho máximo, então cada $T$-fronteira da família é bicrítica.

Demonstração. Seja $\mathcal{F}$ uma família disjunta de $T$-fronteiras de valor máximo e de tamanho máximo. Suponha que uma $T$-fronteira $\delta(\mathcal{P}) \in \mathcal{F}$ não é bicrítica. Seja $H$ o grafo de fronteiras de $\delta(\mathcal{P})$. Cada parte $C \in \mathcal{P}$ denota o vértice de $H$ obtido da contração de $C$, isto é, $V_{H}=\mathcal{P}$. Como $H$ não é bicrítico, então pela afirmação (6.19) existe um subconjunto $S \subseteq \mathcal{P}$ tal que $q_{\mathcal{P}}(H-S)>|S|-2$ e $|S| \geq 2$. Ademais, como $|\mathcal{P}|$ é par, temos que $q_{\mathcal{P}}(H-S) \equiv|S|(\bmod 2)$. Logo,

$$
q_{\mathcal{P}}(H-S) \geq|S| \geq 2 .
$$

Seja $K$ um componente ímpar de $H-S$. Defina uma partição $\mathcal{P}_{K}$ de $V_{H}=\mathcal{P}$ da seguinte forma. Para cada vértice de $K$, crie uma parte de $\mathcal{P}_{K}$ que é um conjunto unitário contendo esse vértice. Por fim, crie uma parte de $\mathcal{P}_{K}$ que contém todos os vértices de $H$ fora de $K$. Tome

$$
\mathcal{P}_{K}^{\prime}:=\left\{\bigcup X: X \in \mathcal{P}_{K}\right\} .
$$

Note que $\mathcal{P}_{K}^{\prime}$ é uma $T$-partição, e portanto $\delta_{G}\left(\mathcal{P}_{K}^{\prime}\right)$ é uma $T$-fronteira. Além disso, o valor da $T$-fronteira $\delta_{G}\left(\mathcal{P}_{K}^{\prime}\right)$ é $(|K|+1) / 2$.

Seja $K$ um componente par de $H-S$. Defina uma partição $\mathcal{P}_{K}$ de $V_{H}=\mathcal{P}$ da seguinte forma. Escolha um vértice $u$ de $K$ que é vizinho de $S$. Para cada vértice de $K \backslash\{u\}$, crie uma parte de $\mathcal{P}_{K}$ que é um conjunto unitário contendo esse vértice. Por fim, crie uma parte de $\mathcal{P}_{K}$ que contém todos os vértices de $H$ fora de $K \backslash\{u\}$. Defina $\mathcal{P}_{K}^{\prime}$ como em (6.21) e note que $\mathcal{P}_{K}^{\prime}$ é uma $T$-partição e que $\delta_{G}\left(\mathcal{P}_{K}^{\prime}\right)$ é uma $T$-fronteira de valor $|K| / 2$.

Tome $\mathcal{F}^{\prime}:=\left\{\delta\left(\mathcal{P}_{K}^{\prime}\right): K\right.$ é um componente de $\left.H-S\right\}$. Observe que $\mathcal{F}^{\prime}$ é uma família disjunta de $T$-fronteiras tal que $\delta_{G}\left(\mathcal{P}_{K}^{\prime}\right) \subseteq \delta_{G}(\mathcal{P})$ para cada $\delta_{G}\left(\mathcal{P}_{K}^{\prime}\right) \in \mathcal{F}^{\prime}$. Ademais, o valor de $\mathcal{F}^{\prime}$ é

$$
\frac{1}{2}\left(|H-S|+q_{\mathcal{P}}(H-S)\right) \geq \frac{1}{2}|H|=\frac{1}{2}|\mathcal{P}|,
$$

ou seja, não é inferior ao valor da $T$-fronteira $\delta_{G}(\mathcal{P})$. Além disso, $\left|\mathcal{F}^{\prime}\right| \geq q_{\mathcal{P}}(H-S) \geq|S| \geq 2$. Mas então $\mathcal{F}^{\prime \prime}:=\left(\mathcal{F} \backslash\left\{\delta_{G}(\mathcal{P})\right\}\right) \cup \mathcal{F}^{\prime}$ é uma família disjunta de $T$-fronteiras de valor máximo e com $\left|\mathcal{F}^{\prime \prime}\right|>|\mathcal{F}|$, contradizendo a escolha de $\mathcal{F}$. 
Vamos ver agora o lema de Sebő sobre grafos bicríticos:

\section{Lema $6.2 \beta$}

Seja $G=(V, E)$ um grafo bicrítico com $|V| \geq 4$. Então existe uma partição $\left\{V_{1}, V_{2}, V_{3}, V_{4}\right\}$ de $V$ tal que, para cada $i$, o subgrafo induzido $G\left[V_{i}\right]$ é conexo e $V_{i}$ é ímpar e, para quaisquer $i, j \in\{1,2,3,4\}$ distintos, existe uma aresta entre $V_{i}$ e $V_{j}$.

Demonstração. Vamos encontrar em $G$ um circuito ímpar $C$ que será a base de construção das partes $V_{1}, V_{2}$ e $V_{3}$, sendo que a parte $V_{4}$ virá de algum componente de $G-V_{C}$.

É claro que $G$ possui um emparelhamento perfeito. Seja $M$ um emparelhamento perfeito de $G$ e $u v \in M$. Tome $M_{u v}:=M \backslash\{u v\}$. Como $|V| \geq 4$ e $G$ é bicrítico, o vértice $u$ tem um vizinho $z$ distinto de $v$. Seja $M_{v z}$ um emparelhamento perfeito de $G-v-z$. É claro que um dos componentes do grafo $\left(V, M_{u v} \triangle M_{v z}\right)$ é um caminho $P_{u z}$ entre $u$ e $z$. Tome o circuito $C:=P_{u z}+u z$. Esse circuito será a base da construção da nossa partição $\left\{V_{1}, V_{2}, V_{3}, V_{4}\right\}$ de $V$.

Nomeie os vértices de $C$ como $u_{0}, \ldots, u_{k}$ em sua ordem cíclica de maneira que $u_{0}=u \mathrm{e}$ $u_{i} u_{i+1} \in M$ para todo $i$ ímpar. Observe que, pela existência do emparelhamento perfeito $M$, que possui a aresta $u v$, o componente $K$ de $G-V_{C}$ que contém o vértice $v$ é ímpar e todos os demais componentes são pares.

Afirmamos que $K$ tem pelo menos 3 vizinhos em $C$. De fato, se $u$ é o único vizinho de $K$ em $C$, então $G-u-u_{1}$ tem um componente ímpar e portanto $G$ não é bicrítico, uma contradição. Além disso, pela afirmação (6.19), o componente ímpar $K$ não pode ter somente 2 vizinhos em $C$.

Sejam $u_{0}, u_{i}$ e $u_{j}$ vizinhos do componente $K$, com $0<i<j$. Se existe uma aresta $u_{h} u_{h+1} \in M$ tal que $i \leq h<j$, defina $V_{1}^{\prime}:=\left\{u_{1}, \ldots, u_{h}\right\}, V_{2}^{\prime}:=\left\{u_{h+1}, \ldots, u_{k}\right\}$ e $V_{3}^{\prime}:=\left\{u_{0}\right\}$. Caso contrário, temos que $j=i+1$ e $i$ é par. Defina então $V_{1}^{\prime}:=\left\{u_{i}\right\}, V_{2}^{\prime}:=\left\{u_{j}\right\}$ e $V_{3}^{\prime}:=V_{C} \backslash\left\{u_{i}, u_{j}\right\}$.

Note que $\mathcal{V}:=\left\{V_{1}^{\prime}, V_{2}^{\prime}, V_{3}^{\prime}\right\}$ é uma partição de $V_{C}$. Para cada componente par $K^{\prime}$ de $G-V_{C}$, escolha arbitrariamente uma parte $V_{i}^{\prime}$ de $\mathcal{V}$ na qual $K^{\prime}$ possui um vizinho e atribua $s\left(K^{\prime}\right):=i$. Para $i=1,2,3$, defina $V_{i}:=V_{i}^{\prime} \cup \bigcup\left\{K^{\prime}: s\left(K^{\prime}\right)=i\right\}$. É fácil ver que a partição $\left\{V_{1}, V_{2}, V_{3}, V_{4}\right\}$ satisfaz as condições do enunciado.

Um par $(G, T)$ formado por um grafo conexo $G=(V, E)$ e um conjunto $T$ compatível é um graft. Seja $e=u v \in E$. A contração da aresta $e$ no graft $(G, T)$ é a operação que transforma o graft $(G, T)$ no graft $\left(G^{\prime}, T^{\prime}\right)$ tal que $G^{\prime}:=G / e$ e $T^{\prime}$ é definido da seguinte maneira. Tomamos $T^{\prime}:=T \backslash\{u, v\}$ se $\{u, v\}$ é $T$-par e $T^{\prime}:=(T \backslash\{u, v\}) \cup\left\{v_{e}\right\}$ caso contrário.

Se um graft $\left(G^{\prime}, T^{\prime}\right)$ pode ser obtido a partir de um graft $(G, T)$ através de uma seqüência de contrações de arestas, dizemos que $\left(G^{\prime}, T^{\prime}\right)$ é um menor de $(G, T)$. É fácil ver que

$\left(K_{4}, V\left(K_{4}\right)\right)$ é um menor de um graft $(G, T)$ se, e somente se, existe uma $T$-partição $\left\{V_{1}, V_{2}, V_{3}, V_{4}\right\}$ de $V_{G}$ tal que, para quaisquer $i, j \in\{1,2,3,4\}$ distintos, existe uma aresta de $G$ entre $V_{i}$ e $V_{j}$. 
Podemos finalmente provar o caso especial do teorema de SEymour [1977].

\section{Teorema 6.2}

Seja $(G, T)$ um graft. Se $\left(K_{4}, V\left(K_{4}\right)\right)$ não é um menor de $(G, T)$, então o tamanho mínimo de uma $T$-junção é igual ao número máximo de $T$-cortes disjuntos.

Demonstração. Pelo corolário 6.1.9, existem uma $T$-junção $J \subseteq E_{G}$ e uma família disjunta de $T$-fronteiras, digamos $\mathcal{F}$, cujo valor é igual a $|J|$. Escolha uma tal família tal que $|\mathcal{F}|$ é o maior possível. Afirmamos que cada $T$-fronteira $\delta_{G}(\mathcal{P}) \in \mathcal{F}$ é um $T$-corte. Pois suponha que uma $T$-fronteira $\delta_{G}(\mathcal{P}) \in \mathcal{F}$ é tal que $|\mathcal{P}| \geq 4$. Pelo lema $6.2 \alpha$, o grafo de fronteiras $G_{\mathcal{P}}$ de $\mathcal{P}$ é bicrítico. Então a partição $\left\{V_{1}^{\prime}, V_{2}^{\prime}, V_{3}^{\prime}, V_{4}^{\prime}\right\}$ fornecida pelo lema $6.2 \beta$ aplicado a $G_{\mathcal{P}}$ pode ser usada para construirmos uma partição $\left\{V_{1}, V_{2}, V_{3}, V_{4}\right\}$ de $V_{G}$ satisfazendo as condições da afirmação (6.22), de modo que $\left(K_{4}, V\left(K_{4}\right)\right)$ é um menor de $(G, T)$, uma contradição.

\section{Corolário 6.2.1}

Seja $(G, T)$ um graft. Então, para qualquer função peso $w: E_{G} \rightarrow \mathbb{Z}_{\geq 0}$, o peso mínimo de uma $T$-junção é igual ao tamanho máximo de uma família $w$-disjunta de $T$-cortes se, e somente se, $\left(K_{4}, V\left(K_{4}\right)\right)$ não é um menor de $(G, T)$.

Demonstração. Se $\left(K_{4}, V\left(K_{4}\right)\right)$ é um menor de $(G, T)$, então a relação min-max falha para a função peso $w:=\chi^{\delta(\mathcal{P})}$, onde $\mathcal{P}$ é a partição dada pela afirmação (6.22).

Se $\left(K_{4}, V\left(K_{4}\right)\right)$ não é um menor de $(G, T)$, então o resultado segue do teorema 6.2 , contraindo cada aresta de peso nulo e substituindo cada aresta $e$ de peso positivo por um caminho de comprimento $w_{e}$.

\section{Resultados relacionados}

A referência básica para o estudo da teoria de emparelhamentos é o clássico livro de Lovász e Plummer [1986]. Uma referência mais atualizada é o livro de SchriJver [2003, capítulos 16-38].

RIZzI [2000, 1998] obteve provas curtas do teorema 6.1 .5 de emparelhamentos de König e do teorema 6.1.6 de coloração de arestas de König.

Vamos discutir agora algoritmos para o problema do emparelhamento máximo em grafos arbitrários. Existem basicamente três tipos de algoritmos para esse problema.

O primeiro deles envolve a busca por caminhos de aumento. Se $G$ é um grafo e $M$ é um emparelhamento, um caminho de aumento é um caminho $P$ tal que as extremidades de $P$ não são saturadas por $M$ e as arestas de $P$ estão alternadamente dentro e fora de $M$. Note que, se $P$ é um caminho de aumento, então $M \triangle P$ é um emparelhamento maior que $M$. $\mathrm{Na}$ verdade, é fácil provar que um emparelhamento é máximo se, e somente se, não existe nenhum caminho de aumento.

À primeira vista, essa condição de otimalidade parece não ser muito útil do ponto de vista algorítmico, já que não é tão fácil procurar por caminhos de aumento em grafos não-bipartidos sem fazer uma busca exaustiva. Essa dificuldade foi contornada por EDMONDS [1965e] em seu artigo seminal: durante a busca por caminhos de aumento, devemos contrair certos circuitos ímpares, chamados por Edmonds de blossoms. Usando essa idéia, Edmonds obteve o primeiro algoritmo polinomial para o problema do emparelhamento máximo. 
EDMONDS [1965c] desenvolveu também um algoritmo primal-dual fortemente polinomial para o problema do emparelhamento de peso máximo, obtendo como subproduto um sistema de inequações lineares que determina o politopo dos emparelhamentos, isto é, o fecho convexo dos vetores de incidência de emparelhamentos. Cunningham e Marsh [1978] provaram que esse sistema é totalmente dual integral, generalizando assim a fórmula de Tutte-Berge. Uma prova curta desse último resultado foi obtida por SCHRIJVER [1983a].

Um outro tipo de algoritmo é baseado na matriz de Tutte, que descrevemos a seguir. Seja $G=(V, E)$ um grafo. Para cada aresta $e \in E$, crie uma indeterminada $x_{e}$, sendo que as indeterminadas $\left\{x_{e}: e \in E\right\}$ são independentes e comutativas. A matriz de Tutte de $G$ é a matriz $M$ sobre $V \times V$ definida da seguinte forma. A matriz $M$ é anti-simétrica, ou seja, sua transposta é $-M$. Para cada par de vértices $u, v \in V$, tome $M[u, v]:= \pm x_{e}$ se $e=u v \in E$ e $M[u, v]:=0$ caso contrário.

TUTTE [1947] provou que um grafo $G$ possui um emparelhamento perfeito se, e somente se, sua matriz de Tutte é não-singular. Isso implica que o posto da matriz de Tutte é igual ao dobro do tamanho máximo de um emparelhamento. Entretanto, esse resultado não fornece imediatamente um algoritmo polinomial via eliminação gaussiana, pois as matrizes intermediárias da eliminação podem conter entradas exponencialmente grandes. LovÁsz [1979] mostrou que, se substituirmos as indeterminadas da matriz de Tutte por inteiros sorteados aleatoriamente, então com alta probabilidade o posto da matriz resultante será igual ao da matriz de Tutte. Isso fornece um algoritmo probabilístico para descobrir o tamanho máximo de um emparelhamento. GEELEN [2000] apresentou um algoritmo polinomial que substitui deterministicamente as indeterminadas da matriz de Tutte por inteiros entre 1 e $|V|$ de modo que a matriz resultante tenha o mesmo posto da matriz de Tutte. HARvey [2006] apresentou recentemente um algoritmo probabilístico de fácil implementação baseado na matriz de Tutte e que é o assintoticamente mais rápido até o momento.

Um terceiro tipo de algoritmo baseia-se no teorema estrutural de Gallai-Edmonds, que mostra que existe um certo conjunto canônico que atinge o mínimo na fórmula de Tutte-Berge e que revela a estrutura de todos os emparelhamentos máximos de um grafo. Apresentamos a seguir esse resultado obtido independentemente por GALLAI [1963, 1964] e EDMONDS [1965e].

Um grafo $G$ é chamado hipo-emparelhável se $G-v$ possui um emparelhamento perfeito para todo vértice $v \in V_{G}$. Num grafo hipo-emparelhável, cada emparelhamento de tamanho máximo é chamado de quase-perfeito.

\section{Teorema 6.3 (Teorema estrutural de Gallai-Edmonds)}

Seja $G$ um grafo. Defina $D:=\left\{v \in V_{G}\right.$ : algum emparelhamento máximo não satura $\left.v\right\}$, $A:=N(D)$ e $C:=V \backslash(D \cup A)$. Então

(i) $S:=D$ atinge o mínimo em (6.15),

(ii) todo componente de $G[D]$ é hipo-emparelhável, e

(iii) todo emparelhamento máximo de $G$ é formado por um emparelhamento perfeito de $G[C]$, um emparelhamento quase-perfeito de cada componente de $G[D]$ e, para cada $v \in A$, uma aresta ligando $v$ a algum vértice de $D$. 
A partição $\{D, A, C\}$ de $V$ obtida nesse teorema pode ser encontrada pelo algoritmo de Edmonds [1965e]. Aqui, entretanto, não estamos interessados num algoritmo que nos forneça essa partição, mas sim num algoritmo cujo funcionamento é baseado no teorema estrutural de Gallai-Edmonds. Algoritmos desse tipo foram chamados por SEBö E SzEgő [2004] de algoritmos estruturais. Vamos descrever brevemente o algoritmo estrutural de LovÁsZ E Plummer [1986].

Ao longo do algoritmo mantemos uma lista $\mathcal{L}$ de emparelhamentos, todos de tamanho $k$ para um certo $k$. Inicialmente, tomamos $\mathcal{L}:=\varnothing$, de modo que $k=0$. Cada passo consiste no seguinte. Primeiro montamos a partição $\{D, A, C\}$ de $V$ como no teorema estrutural de Gallai-Edmonds, porém usando os emparelhamentos da lista $\mathcal{L}$ no lugar do conjunto de todos os emparelhamentos máximos. Por exemplo, $D$ é o conjunto de vértices que não são saturados por algum emparelhamento em $\mathcal{L}$. Se $S:=D$ e $k$ satisfazem com igualdade a fórmula de Tutte-Berge, então cada emparelhamento em $\mathcal{L}$ é máximo. Caso contrário, alguma das conclusões do teorema estrutural de Gallai-Edmonds é violada. Cada violação leva à descoberta de um novo emparelhamento $M^{\prime}$ tal que, ou $\left|M^{\prime}\right|>k$, ou então $\left|M^{\prime}\right|=k$ e $M^{\prime}$ não satura algum vértice fora de $D$. No primeiro caso, tomamos $\mathcal{L}:=\left\{M^{\prime}\right\}$ e $k$ é incrementado. No segundo caso, adicionamos $M^{\prime}$ a $\mathcal{L}$, de modo que a parte $D$ do passo seguinte conterá propriamente a parte $D$ do passo atual. Diferente do que ocorre com o algoritmo de Edmonds, onde a grande dificuldade é encontrar os caminhos de aumento, no algoritmo estrutural os caminhos de aumento "simplesmente aparecem".

Esse algoritmo estrutural não é competitivo com os demais algoritmos em termos de tempo de execução, porém a idéia de usar o teorema estrutural como guia para a elaboração do algoritmo é bastante interessante e foi usada com sucesso para outros problemas, como mencionaremos mais adiante.

Foram propostas diversas extensões de emparelhamentos, como por exemplo o teorema do $f$-fator de TutTe $[1952,1974,1981]$ (veja Anstee [1985] para uma prova algorítmica) e o conceito de jump system de Bouchet e Cunningham [1995]. Lovász [1997] provou uma relação min-max envolvendo jump systems que contém como casos especiais o teorema do $f$-fator e do teorema de intersecção de matróides de Edmonds. Recomendamos a resenha de Cunningham [2002] ao leitor interessado.

Mencionaremos agora brevemente algumas extensões de emparelhamentos com resultados recentes.

Cunningham e Geelen [1997] provaram uma relação min-max envolvendo o conceito de path-matching que contém como casos especiais a fórmula de Tutte-Berge e o teorema de intersecção de matróides de Edmonds. Essa relação min-max foi simplificada por FrANK E Szegő [2002]. Spille E Weismantel [2002] desenvolveram um algoritmo polinomial para path-matchings que é uma generalização comum dos algoritmos de Edmonds para os problemas do emparelhamento máximo e da intersecção de matróides. SpILlE E SzEGő [2004] obtiveram ainda um teorema estrutural do tipo Gallai-Edmonds para path-matchings que generaliza o teorema estrutural de Gallai-Edmonds para emparelhamentos. Veja também os artigos de PAP E SzEGő [2004] e PAP [2004]. 
Uma outra generalização comum para emparelhamentos e intersecção de matróides foi proposta por LAWLER [1976] e envolve o conceito de matroid matching (também conhecido como matroid parity). Esse modelo contém problemas NP-difíceis, mas LovÁsz [1980b, 1981] obteve uma relação min-max e um algoritmo fortemente polinomial para o caso em que o matróide em questão é linear (veja também LovÁsz [1980a]). Recentemente, GeELEn, IwATA E Murota [2003] estenderam esses resultados para delta-matróides (uma generalização de matróides que é um tipo especial de jump system). Geelen e Iwata [2005] relacionaram essa extensão com uma generalização da matriz de Tutte e forneceram uma nova relação min-max e mais aplicações.

No capítulo a seguir vamos estudar uma generalização de emparelhamentos proposta por Chudnovsky, Geelen, Gerards, Goddyn, Lohman e Seymour [2006].

Passemos agora para $T$-junções.

É fácil ver que o problema da $T$-junção de peso mínimo pode ser reduzido ao problema do emparelhamento perfeito de peso mínimo (mesmo que a função peso tenha componentes negativos) e portanto também pode ser resolvido em tempo fortemente polinomial. Isso foi observado por EDMONDS [1965a].

O problema da $T$-junção de peso mínimo está relacionado com diversos outros problemas importantes em otimização combinatória. Por exemplo, o problema do caminho de custo mínimo que liga $r$ a $s$ em grafos não-orientados, restrito ao caso em que os custos podem ser negativos mas não há circuitos de custo negativo, é simplesmente o problema da $\{r, s\}$-junção de peso mínimo.

É claro que o problema chinês do carteiro se reduz ao problema da $T$-junção de peso mínimo em que $T$ é o conjunto de vértices de grau ímpar de $G$. Esse problema também está relacionado com o problema de multifluxos em grafos planares (veja SEYMOUR [1981]) e com o problema do corte máximo em grafos planares (veja HADLOCK [1975] e BARAHONA [1990]).

SEBő $[1990,1986]$ provou um teorema estrutural do tipo Gallai-Edmonds para $T$-junções e elaborou um algoritmo estrutural para $T$-junções baseado nesse teorema.

A caracterização devida a Seymour que provamos na seção 6.4 é um caso especial de um difícil teorema de Seymour [1977], para o qual Guenin [2002] encontrou uma prova curta recentemente. Esse teorema de Seymour também caracteriza os grafts $(G, T)$ que satisfazem a seguinte relação min-max: o tamanho mínimo de um $T$-corte é igual ao número máximo de $T$-junções disjuntas. Uma prova curta dessa caracterização foi obtida por Codato, Conforti e Serafini [1996]. A capacidade mínima de um T-corte pode ser obtida em tempo fortemente polinomial, como mostraram PADBERG E RAO [1982].

Neste texto, derivamos o teorema do casamento de Hall do teorema de emparelhamentos perfeitos de Tutte, e derivamos este do teorema de Frank, Tardos e Sebő. O caminho pode ser revertido, como mostraram ANDERson [1971] e Szigeti [2004]. Isso mostra que esses resultados são, na verdade, equivalentes.

Recomendamos a resenha de FRANK [1996] e a dissertação de REY [1999] para os leitores interessados em mais detalhes sobre $T$-junções, $T$-cortes e funções conservativas. 



\section{Capítulo 7}

\section{$S$-caminhos}

No capítulo 6, estudamos relações min-max para emparelhamentos e para uma extensão desse conceito: as $T$-junções. Neste capítulo, vamos estudar uma outra generalização de emparelhamentos: os $S$-caminhos.

Estamos interessados em resolver problemas do seguinte tipo: dado um grafo, encontre uma coleção máxima de caminhos disjuntos nos vértices e cujas extremidades satisfazem certas restrições. Uma possível restrição, que dá o nome ao capítulo, é a de que as extremidades dos caminhos estejam num certo conjunto $S$. Neste caso, se $S=V$, esse é exatamente o problema do emparelhamento máximo. Outras variantes são formadas com outras restrições sobre as extremidades e permitindo caminhos internamente disjuntos ou disjuntos nas arestas.

A versão mais geral desse problema é conhecida como o problema de $\mathcal{S}$-caminhos de Mader e consiste no seguinte: dado um grafo e uma partição $\mathcal{S}$ de um subconjunto de vértices $S$, encontre uma coleção máxima de caminhos disjuntos nos vértices tais que as extremidades de cada caminho estejam em partes distintas de $\mathcal{S}$. Mader provou uma relação min-max para esse problema. Por mais de 20 anos, as únicas provas conhecidas para essa relação eram bastante longas e complicadas. Recentemente, Schrijver encontrou uma prova curta para essa relação e uma extensão foi obtida por Chudnovsky, Geelen, Gerards, Goddyn, Lohman e Seymour.

Essa extensão é, na verdade, um arcabouço que fornece relações min-max para diversas variantes do problema dos $S$-caminhos. Uma novidade desse arcabouço é o fato de que as arestas do grafo são rotuladas por elementos de um grupo, chamados de pesos. Com isso, podemos definir pesos de caminhos. Se escolhermos o grupo e os rótulos adequadamente, podemos obter uma correspondência entre os $S$-caminhos de peso não-nulo e os tipos de caminhos que queremos encontrar. Neste capítulo, vamos provar a relação min-max obtida por Chudnovsky et al. para o número máximo de $S$-caminhos de peso não-nulo disjuntos nos vértices. A prova não é difícil e baseia-se em alguns conceitos básicos de matróides.

O restante do capítulo está organizado da seguinte maneira: na seção 7.1, estabelecemos a noção de grafos rotulados por elementos de um grupo e definimos $S$-caminhos não-nulos; na seção 7.2, construímos um matróide a partir de $S$-caminhos; na seção 7.3, provamos um lema sobre certas triplas, chamadas de críticas; na seção 7.4, provamos a relação min-max para o número máximo de $S$-caminhos não-nulos disjuntos nos vértices; finalmente, na seção 7.5, apresentamos alguns casos especiais dessa relação, dentre os quais o teorema dos $\mathcal{S}$-caminhos disjuntos de Mader. 


\subsection{Um grafo rotulado por um grupo}

Seja $\Gamma$ um grupo. Usamos notação aditiva, mas notamos que $\Gamma$ não precisa ser abeliano.

Seja $D=(V, A)$ um grafo orientado e $\gamma: A \rightarrow \Gamma$ uma função peso. Se $a=u v \in A$, denotamos $\gamma(a, v):=\gamma_{a}$ e $\gamma(a, u):=-\gamma_{a}$.

Seja $P=\left\langle v_{0}, a_{1}, v_{1}, \ldots, a_{k}, v_{k}\right\rangle$ um pseudocaminho em $D$. Definimos o peso de $P$ como $\gamma(P):=\sum_{i=1}^{k} \gamma\left(a_{i}, v_{i}\right)$, ou seja, somamos os pesos dos arcos diretos de $P$ e o oposto dos pesos dos arcos reversos de $P$. Se o grupo $\Gamma$ não é abeliano, tomamos a soma na ordem em que percorremos o pseudocaminho. Um pseudocaminho $P$ é não-nulo se $\gamma(P) \neq 0$.

Seja $S \subseteq V$. Um $S$-caminho é um pseudocaminho com pelo menos um arco e com as duas extremidades em $S$.

Denote por $\nu(D, S, \gamma)$ o número máximo de $S$-caminhos não-nulos disjuntos nos vértices. Vamos usar essa notação mesmo quando $\gamma$ é uma função de um superconjunto de $A$ para $\Gamma$.

Ao longo das próximas seções vamos provar uma relação min-max para $\nu(D, S, \gamma)$ obtida por Chudnovsky, Geelen, Gerards, Goddyn, Lohman e Seymour [2006].

Uma operação fundamental será a de deslocar o peso dos arcos incidentes num certo vértice, que descrevemos a seguir.

Seja $x \in V$ e $\beta \in \Gamma$. O deslocamento da função peso $\gamma$ de $\beta$ em $x$ é a operação que transforma $\gamma$ na função peso $\gamma^{\prime}$ definida como

$$
\gamma_{a}^{\prime}:= \begin{cases}\gamma_{a}+\beta, & \text { se } v=x \\ -\beta+\gamma_{a}, & \text { se } u=x \\ \gamma_{a}, & \text { caso contrário, }\end{cases}
$$

para cada arco $a=u v \in A$. Note que, se $x \notin S$, então $\gamma^{\prime}(P)=\gamma(P)$ para todo $S$-caminho $P$, mesmo quando o grupo $\Gamma$ não é abeliano.

Se $\gamma^{\prime}$ é uma função peso obtida a partir de $\gamma$ através de deslocamentos em vértices fora de $S$, dizemos que $\gamma^{\prime}$ é $S$-equivalente a $\gamma$.

Vamos definir o peso de pseudocircuitos de forma análoga ao peso de pseudocaminhos. Seja $C=\left\langle v_{0}, a_{1}, v_{1}, \ldots, a_{k}, v_{k}\right\rangle$ um pseudocircuito em $D$. Definimos o peso de $C$ como $\gamma(C):=\sum_{i=1}^{k} \gamma\left(a_{i}, v_{i}\right)$. Um pseudocircuito $C$ é não-nulo se $\gamma(C) \neq 0$. Observe que o deslocamento da função peso em qualquer vértice não altera o peso de nenhum pseudocircuito.

Dizemos que $B \subseteq A$ é $S$-balanceado (com relação a $\gamma$ ) se o conjunto $B$ não contém $S$-caminhos não-nulos nem pseudocircuitos não-nulos. Denote por $V_{B}$ o conjunto dos vértices que são pontas de algum arco de $B$.

Como aquecimento para as seções seguintes, vamos provar a seguinte proposição:

\section{Proposição 7.1}

Seja $D=(V, A)$ um grafo orientado, $\Gamma$ um grupo e $\gamma: A \rightarrow \Gamma$ uma função peso. Seja $S \subseteq V$. Então $B \subseteq A$ é $S$-balanceado se, e somente se, existe uma função peso $\gamma^{\prime}: A \rightarrow \Gamma$ que é $S$-equivalente a $\gamma$ tal que $\gamma_{a}^{\prime}=0$ para todo $a \in B$.

Demonstração. Como o deslocamento da função peso em vértices fora de $S$ não altera o peso de nenhum $S$-caminho, é claro que essa condição é suficiente. 
Vamos provar que ela é necessária por indução em $\left|V_{B} \backslash S\right|$. Se $V_{B} \subseteq S$, então cada arco de $B$ tem as duas pontas em $S$, de modo que $\gamma_{a}=0$ para todo $a \in B$. Suponha então que existe $v \in V_{B} \backslash S$.

Seja $R$ o conjunto de todos os vértices acessíveis a partir de $v$ através de pseudocaminhos com todos os arcos em $B$. Se $R \cap S=\varnothing$, tome $S^{\prime}:=S \cup\{v\}$. É óbvio que $B$ é $S^{\prime}$-balanceado.

Suponha então que $R \cap S \neq \varnothing$. Seja $P=\left\langle v_{0}, a_{1}, v_{1}, \ldots, a_{k}, v_{k}\right\rangle$ um pseudocaminho contido em $B$ de comprimento mínimo que liga $v=v_{0}$ a algum vértice de $S$. Tome $s:=v_{k}, u:=v_{k-1}$ e $a:=a_{k}$. É evidente que $u \in V_{B} \backslash S$. Desloque a função peso $\gamma$ em $u$ de maneira que a função peso resultante $\gamma^{\prime}$ satisfaça $\gamma_{a}^{\prime}=0$. Tome $S^{\prime}:=S \cup\{u\}$. Como $B$ não contém nenhum $S$-caminho não-nulo, então $B$ também não contém nenhum pseudocaminho não-nulo ligando $u$ a um vértice de $S \backslash\{s\}$. Como $B$ não contém nenhum pseudocircuito não-nulo, então $B$ também não contém nenhum pseudocaminho não-nulo ligando $u$ a $s$. Concluímos que $B$ é $S^{\prime}$-balanceado com relação a $\gamma^{\prime}$.

Em ambos os casos, $B$ é $S^{\prime}$-balanceado com relação a uma função $S$-equivalente a $\gamma$ e $\left|V_{B} \backslash S^{\prime}\right|<\left|V_{B} \backslash S\right|$. Agora o resultado segue por indução.

A demonstração acima fornece um algoritmo eficiente que decide se um conjunto de arcos é $S$-balanceado.

\subsection{Bases de um matróide de $S$-caminhos}

Nesta seção, vamos mostrar que uma certa coleção de conjuntos construída a partir de $S$-caminhos forma a coleção de bases de um matróide.

Seja $D=(V, A)$ um grafo orientado, $\Gamma$ um grupo e $\gamma: A \rightarrow \Gamma$ uma função peso. Seja $S \subseteq V$. Defina a deficiência de $S \operatorname{como} \operatorname{def}(D, S, \gamma):=|S|-2 \nu(D, S, \gamma)$.

Seja $\mathcal{P}$ um conjunto de pseudocaminhos disjuntos nos vértices. Dizemos que $\mathcal{P}$ é uma $S$-coleção se satisfaz as seguintes condições:

(i) cada vértice de $S$ é extremidade de algum pseudocaminho em $\mathcal{P}$,

(ii) a extremidade inicial de cada pseudocaminho de $\mathcal{P}$ está em $S$,

(iii) cada pseudocaminho de $\mathcal{P}$ que é um $S$-caminho é não-nulo.

Seja $\mathcal{P}$ uma $S$-coleção. Dizemos que um pseudocaminho de $\mathcal{P}$ é solto se sua extremidade final não está em $S$. Observe então que cada pseudocaminho de $\mathcal{P}$ ou é solto ou então é um $S$-caminho não-nulo. Dizemos que $\mathcal{P}$ é ótima se contém $\nu(D, S, \gamma) S$-caminhos. Denote por $A(\mathcal{P})$ o conjunto $\bigcup_{P \in \mathcal{P}} A_{P}$.

Vamos definir agora o conjunto-base do nosso matróide.

Tome $\Gamma^{\prime}:=\{\gamma(P): P$ é um pseudocaminho em $D\}$ e note que $\Gamma^{\prime}$ é finito mesmo quando $\Gamma$ é infinito. Agora tome $E:=\{(s, 0): s \in S\} \cup\left\{(v, \gamma): v \in V \backslash S, \gamma \in \Gamma^{\prime}\right\}$.

Vamos definir agora um matróide sobre $E$. Seja $\mathcal{P}$ uma $S$-coleção. Denote por $B(\mathcal{P})$ o conjunto de todos os pares $(v, \gamma(P))$, onde $P$ é um pseudocaminho solto de $\mathcal{P}$ e $v$ é a extremidade final de $P$. Tome $\mathcal{B}:=\{B(\mathcal{P}): \mathcal{P}$ é uma $S$-coleção ótima $\}$. 


\section{Lema $7.2 \alpha$}

A coleção $\mathcal{B}$ é a coleção de bases de um matróide sobre $E$.

Demonstração. Basta provarmos que a coleção $\mathcal{B}$ satisfaz as condições (1.16) do teorema 1.11. É evidente que $\mathcal{B}$ satisfaz (1.16)(i). Suponha que $\mathcal{B}$ não satisfaz (1.16)(ii). Então

existem $S$-coleções ótimas $\mathcal{P}$ e $\mathcal{P}^{\prime}$ e $(u, \alpha) \in B(\mathcal{P}) \backslash B\left(\mathcal{P}^{\prime}\right)$ tais que, para cada $\left(u^{\prime}, \alpha^{\prime}\right) \in B\left(\mathcal{P}^{\prime}\right) \backslash B(\mathcal{P})$, vale que $B(\mathcal{P})-(u, \alpha)+\left(u^{\prime}, \alpha^{\prime}\right) \notin \mathcal{B}$.

Escolha tais $\mathcal{P}$ e $\mathcal{P}^{\prime}$ de modo que

$$
\left|A(\mathcal{P}) \backslash A\left(\mathcal{P}^{\prime}\right)\right|
$$

seja o menor possível.

Vamos usar repetidamente a seguinte afirmação:

Não existe uma $S$-coleção ótima $\mathcal{P}^{\prime \prime}$ tal que $B(\mathcal{P}) \backslash B\left(\mathcal{P}^{\prime \prime}\right)=\{(u, \alpha)\}$ e $\left|A\left(\mathcal{P}^{\prime \prime}\right) \backslash A\left(\mathcal{P}^{\prime}\right)\right|<\left|A(\mathcal{P}) \backslash A\left(\mathcal{P}^{\prime}\right)\right|$.

Pois suponha o contrário. Como $\mathcal{P}$ e $\mathcal{P}^{\prime \prime}$ são $S$-coleções ótimas, então $|B(\mathcal{P})|=\left|B\left(\mathcal{P}^{\prime \prime}\right)\right|$, de modo que existe um único elemento em $B\left(\mathcal{P}^{\prime \prime}\right) \backslash B(\mathcal{P})$. Seja $\left(u^{\prime \prime}, \alpha^{\prime \prime}\right)$ tal elemento. Como $B\left(\mathcal{P}^{\prime \prime}\right)=B(\mathcal{P})-(u, \alpha)+\left(u^{\prime \prime}, \alpha^{\prime \prime}\right) \in \mathcal{B}$, então $\left(u^{\prime \prime}, \alpha^{\prime \prime}\right) \notin B\left(\mathcal{P}^{\prime}\right)$. Pela minimalidade de (7.3), existe $\left(u^{\prime}, \alpha^{\prime}\right) \in B\left(\mathcal{P}^{\prime}\right) \backslash B\left(\mathcal{P}^{\prime \prime}\right)$ tal que $B\left(\mathcal{P}^{\prime \prime}\right)-\left(u^{\prime \prime}, \alpha^{\prime \prime}\right)+\left(u^{\prime}, \alpha^{\prime}\right) \in \mathcal{B}$. Mas então temos que $B(\mathcal{P})-(u, \alpha)+\left(u^{\prime}, \alpha^{\prime}\right)=B\left(\mathcal{P}^{\prime \prime}\right)-\left(u^{\prime \prime}, \alpha^{\prime \prime}\right)+\left(u^{\prime}, \alpha^{\prime}\right) \in \mathcal{B}$, contradizendo (7.2). Isso conclui a prova da afirmação (7.4).

Vamos adotar a seguinte notação. Se $P=\left\langle v_{0}, a_{1}, v_{1}, \ldots, a_{k}, v_{k}\right\rangle$ é um pseudocaminho e $i, j \in\{0, \ldots, k\}$, então $P\left[v_{i}, v_{j}\right]$ denota o pseudocaminho em $P$ que vai de $v_{i}$ a $v_{j}$.

Seja $P=\left\langle v_{0}, a_{1}, v_{1}, \ldots, a_{k}, v_{k}\right\rangle$ o pseudocaminho de $\mathcal{P}$ que termina em $u$, isto é, $v_{k}=u$. Seja $P^{\prime}$ o pseudocaminho de $\mathcal{P}^{\prime}$ que tem $v_{0} \in S$ como extremidade. Vamos mostrar que

$$
A_{P} \subseteq A_{P^{\prime}}
$$

Suponha o contrário. Escolha o menor $i$ tal que $a_{i} \notin A_{P^{\prime}}$. Tome $\mathcal{P}^{\prime \prime}:=\mathcal{P}-P+P\left[v_{0}, v_{i-1}\right]$ e note que $\mathcal{P}^{\prime \prime}$ satisfaz as condições de (7.4). Essa contradição conclui a prova de (7.5).

Suponha que $P^{\prime}=\left\langle v_{0}^{\prime}, a_{1}^{\prime}, v_{1}^{\prime}, \ldots, a_{\ell}^{\prime}, v_{\ell}^{\prime}\right\rangle$. Podemos supor que $v_{0}=v_{0}^{\prime}$. Vamos mostrar que

$$
\text { existe } Q \in \mathcal{P} \backslash\{P\} \text { tal que } V_{Q} \cap V_{P^{\prime}} \neq \varnothing \text {. }
$$

Caso contrário, tome $\mathcal{P}^{\prime \prime}:=\mathcal{P}-P+P^{\prime}$. Note que, como $\mathcal{P}$ é uma $S$-coleção ótima, então $\mathcal{P}^{\prime \prime}$ também o é, e $P^{\prime}$ é um pseudocaminho solto. Mas então $\left(v_{\ell}^{\prime}, \gamma\left(P^{\prime}\right)\right) \in B\left(\mathcal{P}^{\prime}\right) \backslash B(\mathcal{P})$ e $B(\mathcal{P})-(u, \alpha)+\left(v_{\ell}^{\prime}, \gamma\left(P^{\prime}\right)\right)=B\left(\mathcal{P}^{\prime \prime}\right) \in \mathcal{B}$, contradizendo (7.2). Essa contradição conclui a prova de (7.6).

Seja $i$ mínimo tal que $v_{i}^{\prime} \in V_{Q}$ para algum pseudocaminho $Q \in \mathcal{P} \backslash\{P\}$. Suponha que $Q=\left\langle x_{0}, b_{1}, x_{1}, \ldots, b_{m}^{\prime}, x_{m}\right\rangle$ e que $v_{i}^{\prime}=x_{j}$. Note que $Q$ pode ser um pseudocaminho solto ou um $S$-caminho. A seguir, consideramos essas duas possibilidades separadamente. 
Primeiro suponha que $Q$ é um pseudocaminho solto. Podemos supor que $x_{0} \in S$. Seja $P_{1}$ o $S$-caminho que vai de $v_{0}^{\prime}$ a $x_{0}$ em $P^{\prime} \cup Q$ e $P_{2}$ o pseudocaminho que vai de $v_{0}^{\prime}$ a $x_{m}$ em $P^{\prime} \cup Q$. Como $\mathcal{P}$ é uma $S$-coleção ótima, então $\gamma\left(P_{1}\right)=0$. Portanto, $\gamma\left(P^{\prime}\left[v_{0}^{\prime}, v_{i}^{\prime}\right]\right)=\gamma\left(Q\left[x_{0}, x_{j}\right]\right)$, de modo que $\gamma\left(P_{2}\right)=\gamma(Q)$. Seja $Q^{\prime}$ o pseudocaminho formado unicamente pelo vértice $x_{0}$. Tome a $S$-coleção $\mathcal{P}^{\prime \prime}-P-Q+P_{2}+Q^{\prime}$. É claro que $\mathcal{P}^{\prime \prime}$ é uma $S$-coleção ótima. Como $\gamma\left(P_{2}\right)=\gamma(Q)$, então $B(\mathcal{P}) \backslash B\left(\mathcal{P}^{\prime \prime}\right)=\{(u, \alpha)\}$. Porém, $P_{1}$ é um $S$-caminho que tem peso nulo, de modo que $P^{\prime} \neq P_{1}$, já que $\mathcal{P}^{\prime}$ é uma $S$-coleção. Isso implica que existe um arco de $Q\left[x_{0}, x_{j}\right]$ que não está em $A\left(\mathcal{P}^{\prime \prime}\right)$. Mas então $\left|A\left(\mathcal{P}^{\prime \prime}\right) \backslash A\left(\mathcal{P}^{\prime}\right)\right|<\left|A(\mathcal{P}) \backslash A\left(\mathcal{P}^{\prime}\right)\right|$, contradizendo a afirmação (7.4).

Concluímos que $Q$ é um $S$-caminho. Seja $P_{1}$ o pseudocaminho que vai de $v_{0}^{\prime}$ a $x_{0} \mathrm{em}$ $P^{\prime}\left[v_{0}^{\prime}, v_{i}^{\prime}\right] \cup Q\left[x_{0}, x_{j}\right]$. Seja $P_{2}$ o pseudocaminho que vai de $v_{0}^{\prime}$ a $x_{m}$ em $P^{\prime}\left[v_{0}^{\prime}, v_{i}^{\prime}\right] \cup Q\left[x_{j}, x_{m}\right]$. Denote por $P_{2}^{-1}$ o reverso do pseudocaminho $P_{2}$, ou seja, o pseudocaminho em $P_{2}$ que vai de $x_{m}$ a $v_{0}^{\prime}$. Observe que $\gamma\left(P_{1}\right)+\gamma(Q)+\gamma\left(P_{2}^{-1}\right)=0$ e $\gamma(Q) \neq 0$, de modo que ou $\gamma\left(P_{1}\right) \neq 0$ ou $\gamma\left(P_{2}\right) \neq 0$. Podemos supor que

$$
\gamma\left(P_{2}\right) \neq 0 \text { e } P_{1} \neq P^{\prime}
$$

De fato, se $\gamma\left(P_{1}\right) \neq 0$ e $P_{2} \neq P^{\prime}$, basta trocarmos $P_{1}$ e $P_{2}$ e revertermos o pseudocaminho $Q$ para que ele vá de $x_{m}$ a $x_{0}$. Se $\gamma\left(P_{1}\right)=0$, então $\gamma\left(P_{2}\right) \neq 0$. Além disso, $P^{\prime}$ ou é um pseudocaminho solto ou um $S$-caminho tal que $\gamma\left(P^{\prime}\right) \neq 0$, de modo que $P_{1} \neq P^{\prime}$. Já se $P_{2}=P^{\prime}$, então $P_{1} \neq P^{\prime}$ e $\gamma\left(P_{2}\right) \neq 0$, pois $P^{\prime}$ está na $S$-coleção $\mathcal{P}^{\prime}$.

Tome $\mathcal{P}^{\prime \prime}:=\mathcal{P}-P-Q+P_{2}+Q^{\prime}$, onde $Q^{\prime}$ é o pseudocaminho formado unicamente pelo vértice $x_{0}$. Note que $\mathcal{P}^{\prime \prime}$ é uma $S$-coleção ótima e que $B(\mathcal{P}) \backslash B\left(\mathcal{P}^{\prime \prime}\right)=\{(u, \alpha)\}$. Além disso, como $P_{1} \neq P^{\prime}$, então existe um arco de $Q\left[x_{0}, x_{j}\right]$ que não está em $A\left(\mathcal{P}^{\prime \prime}\right)$, o que implica que $\left|A\left(\mathcal{P}^{\prime \prime}\right) \backslash A\left(\mathcal{P}^{\prime}\right)\right|<\left|A(\mathcal{P}) \backslash A\left(\mathcal{P}^{\prime}\right)\right|$. Isso contradiz a afirmação (7.4).

Essa contradição prova que a afirmação (7.2) é falsa, e portanto a coleção $\mathcal{B}$ satisfaz a condição (1.16)(ii). Agora o lema segue do teorema 1.11.

\subsection{Triplas críticas}

Seja $M=(E, \mathcal{I})$ um matróide. Lembre-se de que um laço é um elemento de $E$ que não está em nenhuma base de $M$. Seja $E^{\prime}$ o conjunto de todos os elementos de $E$ que não são laços. Dados $e, f \in E^{\prime}$ distintos, dizemos que e é paralelo a $f$ se nenhuma base contém ambos $e$ e $f$. Pela proposição 1.13, a relação "é paralelo a" é transitiva.

Seja $D=(V, A)$ um grafo orientado, $\Gamma$ um grupo e $\gamma: A \rightarrow \Gamma$ uma função peso. Seja $S \subseteq V$. Denote por $A_{0}(S, \gamma)$ o conjunto dos arcos de peso nulo com ambas as pontas em $S$. Dizemos que a tripla $(D, S, \gamma)$ é crítica se as seguintes condições são satisfeitas:

(i) $D$ é fracamente conexo,

(ii) $\nu(D-v, S \backslash\{v\}, \gamma)=\nu(D, S, \gamma)$ para todo $v \in V$,

(iii) $\nu\left(D, S \cup\{v\}, \gamma^{\prime}\right)>\nu(D, S, \gamma)$ para todo $v \in V \backslash S$ e toda função peso $\gamma^{\prime} \neq \gamma$ que é $S$-equivalente a $\gamma$,

(iv) $A_{0}(S, \gamma)=\varnothing$. 
O seguinte lema é uma extensão de um resultado de GALLAI [1961]:

\section{Lema $7.2 \beta$}

Seja $(D, S, \gamma)$ uma tripla crítica. Então $\operatorname{def}(D, S, \gamma)=1$.

Demonstração. Suponha que $D=(V, A)$. Defina os conjuntos $\Gamma^{\prime}, E, E^{\prime}$ e a coleção $\mathcal{B}$ como na seção 7.2. Pelo lema $7.2 \alpha$, a coleção $\mathcal{B}$ é a coleção de bases de um matróide. Seja $M=(E, \mathcal{I})$ tal matróide. A prova consiste de uma série de afirmações.

Seja $a \in A$ um arco com pontas $u, v \in V$ e sejam $(u, \alpha),(v, \beta) \in E^{\prime}$.

Se $\alpha+\gamma_{a}-\beta \neq 0$, então $(u, \alpha)$ é paralelo a $(v, \beta)$.

Vamos provar a contrapositiva. Seja $\mathcal{P}$ uma $S$-coleção ótima tal que $(u, \alpha),(v, \beta) \in B(\mathcal{P})$. Sejam $P_{u}$ e $P_{v}$ os pseudocaminhos de $\mathcal{P}$ que passam por $u$ e por $v$, respectivamente. Note que $\left(P_{u} \cup P_{v}\right)+a$ contém um $S$-caminho $P$ que vai da extremidade inicial de $P_{u}$ à extremidade inicial de $P_{v}$. Mas como $\mathcal{P}$ é ótimo, então $\gamma(P)=\alpha+\gamma_{a}-\beta=0$. Isso conclui a prova da afirmação (7.9).

$$
\text { Para cada } s \in S \text {, temos que }(s, 0) \in E^{\prime} \text {. }
$$

De fato, por (7.8)(ii), vale que $\nu(D-s, S \backslash\{s\}, \gamma)=\nu(D, S, \gamma)$, ou seja, existe uma coleção $\mathcal{P}$ de $\nu(D, S, \gamma) S$-caminhos não-nulos disjuntos nos vértices tal que nenhum pseudocaminho de $\mathcal{P}$ passa por $s$. Adicionando pseudocaminhos formados por um único vértice de $S$ a $\mathcal{P}$, obtemos uma $S$-coleção ótima $\mathcal{P}^{\prime}$ tal que $(s, 0) \in B\left(\mathcal{P}^{\prime}\right)$. Isso conclui a prova de (7.10).

Para qualquer $v \in V \backslash S$, existem elementos distintos $(v, \alpha),\left(v, \alpha^{\prime}\right) \in E^{\prime}$.

De fato, seja $\beta \in \Gamma$. Desloque a função peso $\gamma$ de $\beta$ em $v$ e seja $\gamma^{\prime}$ a função peso resultante. Tome $S^{\prime}:=S \cup\{v\}$. Por (7.8)(iii), vale que $\nu\left(D, S^{\prime}, \gamma^{\prime}\right)=\nu(D, S, \gamma)+1$. Seja $\mathcal{P}$ uma $S^{\prime}$-coleção ótima com relação a $\gamma^{\prime}$. Note que $v$ é uma extremidade de algum $S^{\prime}$-caminho de $\mathcal{P}$. Seja $P \in \mathcal{P}$ tal $S^{\prime}$-caminho. Podemos supor que $v$ é a extremidade final de $P$. Então $\mathcal{P}$ é uma $S$-coleção ótima com $\gamma^{\prime}(P)=\gamma(P)+\beta \neq 0$. Note que $(v, \gamma(P)) \in E^{\prime}$ e $\gamma(P) \neq-\beta$. Como $\beta \in \Gamma$ foi escolhido arbitrariamente, segue que devem existir elementos distintos $(v, \alpha),\left(v, \alpha^{\prime}\right) \in E^{\prime}$. Isso conclui a prova da afirmação (7.11).

Seja $a=u v \in A$. Então existem $\left(u, \alpha_{u}\right),\left(v, \alpha_{v}\right) \in E^{\prime}$ tais que $\left(u, \alpha_{u}\right)$ é paralelo a $\left(v, \alpha_{v}\right)$.

Primeiro suponha que $u, v \in S$. Tome $\alpha_{u}:=\alpha_{v}:=0$. Pela condição (7.8)(iv), temos que $0 \neq \gamma_{a}=\alpha_{u}+\gamma_{a}-\alpha_{v}$. Agora a afirmação segue de (7.9). Podemos supor então que $u \notin S$ ou $v \notin S$. Vamos supor que $v \notin S$, já que o outro caso é análogo. Pelas afirmações (7.10) e (7.11), existe $\alpha_{u} \in \Gamma$ tal que $\left(u, \alpha_{u}\right) \in E^{\prime}$. Pela afirmação (7.11), existe $\alpha_{v} \in \Gamma$ tal que $\left(v, \alpha_{v}\right) \in E^{\prime}$ e $\alpha_{v} \neq \alpha_{u}+\gamma_{a}$. Logo, pela afirmação (7.9), temos que $\left(u, \alpha_{u}\right)$ é paralelo a $\left(v, \alpha_{v}\right)$. Isso conclui a prova da afirmação (7.12). 
Podemos finalmente provar o lema.

Para cada $v \in V$, abrevie $E_{v}^{\prime}:=\left\{(u, \alpha) \in E^{\prime}: u=v\right\}$. Seja $\mathcal{P}$ uma $S$-coleção ótima. Como no máximo um pseudocaminho de $\mathcal{P}$ tem $v$ como extremidade, então $\left|B(\mathcal{P}) \cap E_{v}^{\prime}\right| \leq 1$, de modo que qualquer elemento de $E_{v}^{\prime}$ é paralelo a qualquer outro elemento de $E_{v}^{\prime}$. Agora, usando a afirmação (7.12) e a condição (7.8)(i), obtemos que cada elemento de $E^{\prime}$ é paralelo a qualquer outro elemento de $E^{\prime}$. Portanto, se $\mathcal{P}$ é uma $S$-coleção ótima, então $|B(\mathcal{P})|=1$, de modo que $\operatorname{def}(D, S, \gamma)=1$, como queríamos.

\subsection{Uma relação min-max para $S$-caminhos não-nulos}

Nesta seção vamos provar uma relação min-max para o número máximo de $S$-caminhos não-nulos disjuntos nos vértices.

Dado um grafo orientado $D$, denote por $\mathcal{K}(D)$ o conjunto dos componentes fracos de $D$.

\section{Teorema 7.2}

Seja $D=(V, A)$ um grafo orientado, $\Gamma$ um grupo e $\gamma: A \rightarrow \Gamma$ uma função peso. Seja $S \subseteq V$. Então existe uma função peso $\gamma^{\prime}$ que é $S$-equivalente a $\gamma$ e existem subconjuntos $X, S^{\prime} \subseteq V$ tais que $S \backslash X \subseteq S^{\prime} \subseteq V \backslash X$ e

$$
\nu(D, S, \gamma)=|X|+\sum\left\{\left\lfloor\frac{1}{2}\left|S^{\prime} \cap K\right|\right\rfloor: K \in \mathcal{K}\left(D-X-A_{0}\left(S^{\prime}, \gamma^{\prime}\right)\right)\right\} .
$$

Demonstração. Seja $X \subseteq V$ maximal tal que $\nu(D-X, S \backslash X, \gamma)=\nu(D, S, \gamma)-|X|$. Dentre todos os pares $\left(S^{\prime}, \gamma^{\prime}\right)$ tais que

(i) $S \backslash X \subseteq S^{\prime} \subseteq V \backslash X$,

(ii) $\gamma^{\prime}$ é $S$-equivalente a $\gamma$, e

(iii) $\nu\left(D-X, S^{\prime}, \gamma^{\prime}\right)=\nu(D-X, S \backslash X, \gamma)$,

escolha um par $\left(S^{\prime}, \gamma^{\prime}\right)$ que maximiza $\left|S^{\prime}\right|$.

Tome $D^{\prime}:=D-X-A_{0}\left(S^{\prime}, \gamma^{\prime}\right)$. Abrevie $\mathcal{K}:=\mathcal{K}\left(D^{\prime}\right)$. Para cada $K \in \mathcal{K}$, abrevie $S_{K}^{\prime}:=S^{\prime} \cap K$. Note que

$$
\nu(D, S, \gamma)=\nu\left(D, S, \gamma^{\prime}\right)=|X|+\sum_{K \in \mathcal{K}} \nu\left(K, S_{K}^{\prime}, \gamma^{\prime}\right)
$$

Observe ainda que, para cada $K \in \mathcal{K}$, a tripla $\left(K, S_{K}^{\prime}, \gamma^{\prime}\right)$ é crítica. Para ver isso, fixe $K \in \mathcal{K}$. As condições (7.8)(i) e (7.8)(iv) são obviamente satisfeitas. As condições (7.8)(ii) e (7.8)(iii) são satisfeitas devido à maximalidade de $X$ e $S^{\prime}$, respectivamente.

Seja $K \in \mathcal{K}$. Pelo lema $7.2 \beta$, temos que $\operatorname{def}\left(K, S_{K}^{\prime}, \gamma^{\prime}\right)=1$, de modo que

$$
\nu\left(K, S_{K}^{\prime}, \gamma^{\prime}\right)=\left\lfloor\frac{1}{2}\left|S_{K}^{\prime}\right|\right\rfloor .
$$

Mas então

$$
\nu(D, S, \gamma)=|X|+\sum_{K \in \mathcal{K}} \nu\left(K, S_{K}^{\prime}, \gamma^{\prime}\right)=|X|+\sum_{K \in \mathcal{K}}\left\lfloor\frac{1}{2}\left|S_{K}^{\prime}\right|\right\rfloor,
$$

como queríamos. 
Agora é fácil derivar a relação min-max para empacotamento de $S$-caminhos não-nulos obtida por Chudnovsky, Geelen, Gerards, Goddyn, Lohman e Seymour [2006]:

\section{Corolário 7.2.1}

Seja $D=(V, A)$ um grafo orientado, $\Gamma$ um grupo e $\gamma: A \rightarrow \Gamma$ uma função peso. Seja $S \subseteq V$. Então

$$
\left.\nu(D, S, \gamma)=\min \left\{|X|+\sum\left\{\left|\frac{1}{2}\right|\left(S \cup V_{B}\right) \cap K \mid\right\rfloor: K \in \mathcal{K}(D-X-B)\right\}\right\}
$$

onde o mínimo é tomado sobre todos os subconjuntos $X \subseteq V$ e todos os subconjuntos de $\operatorname{arcos} B \subseteq A$ que são $S$-balanceados.

Demonstração. Primeiro vamos mostrar que $\nu:=\nu(D, S, \gamma)$ não excede o mínimo. Seja $X \subseteq V$ e $B \subseteq A$ um conjunto $S$-balanceado. Pela proposição 7.1, existe uma função peso $\gamma^{\prime}$ que é $S$-equivalente a $\gamma$ e tal que $\gamma_{a}^{\prime}=0$ para todo $a \in B$. Sejam $P_{1}, \ldots, P_{\nu} S$-caminhos não-nulos com relação a $\gamma^{\prime}$ e disjuntos nos vértices. É evidente que no máximo $|X|$ desses $S$-caminhos intersectam $X$. Os demais são $(S \backslash X)$-caminhos em $D-X$. Logo,

$$
\begin{aligned}
\nu\left(D, S, \gamma^{\prime}\right) & \leq|X|+\nu\left(D-X, S \backslash X, \gamma^{\prime}\right) \\
& \leq|X|+\nu\left(D-X,\left(S \cup V_{B}\right) \backslash X, \gamma^{\prime}\right) \\
& =|X|+\nu\left(D-X-B,\left(S \cup V_{B}\right) \backslash X, \gamma^{\prime}\right) \\
& =|X|+\sum\left\{\nu\left(K,\left(S \cup V_{B}\right) \cap K, \gamma^{\prime}\right): K \in \mathcal{K}(D-X-B)\right\} \\
& \leq|X|+\sum\left\{\left\lfloor\frac{1}{2}\left|\left(S \cup V_{B}\right) \cap K\right|\right\rfloor: K \in \mathcal{K}(D-X-B)\right\} .
\end{aligned}
$$

Como $\nu(D, S, \gamma)=\nu\left(D, S, \gamma^{\prime}\right)$, então $\nu$ não excede o mínimo.

Agora vamos provar que existem $X \subseteq V$ e $B \subseteq A$ tais que $B$ é $S$-balanceado e que satisfazem a relação min-max (7.15).

Pelo teorema 7.2, existe uma função peso $\gamma^{\prime}$ que é $S$-equivalente a $\gamma$ e existem subconjuntos $X, S^{\prime} \subseteq V$ tais que $S \backslash X \subseteq S^{\prime} \subseteq V \backslash X$ e que satisfazem (7.13). Então $B:=A_{0}\left(S^{\prime}, \gamma^{\prime}\right)$ é $S$-balanceado pela proposição 7.1. Além disso, é claro que $\left(S \cup V_{B}\right) \backslash X \subseteq S^{\prime}$. Mas então

$$
\begin{aligned}
&|X|+\sum\left\{\left\lfloor\frac{1}{2}\left|\left(S \cup V_{B}\right) \cap K\right|\right\rfloor: K\right.\in \mathcal{K}(D-X-B)\} \leq \\
&\left.|X|+\sum\left\{\left|\frac{1}{2}\right| S^{\prime} \cap K \mid\right\rfloor: K \in \mathcal{K}(D-X-B)\right\}=\nu(D, S, \gamma),
\end{aligned}
$$

como queríamos. 


\subsection{Conseqüências}

Nesta seção vamos ver algumas aplicações da relação min-max do corolário 7.2.1.

Seja $G=(V, E)$ um grafo e $D=(V, A)$ uma orientação arbitrária de $G$.

Vamos relacionar agora o corolário 7.2.1 à fórmula 6.1.3 de Tutte-Berge. Defina uma função peso $\gamma: A \rightarrow \mathbb{Z}_{2}$ tomando $\gamma(a):=1$ para todo $a \in A$. É óbvio que $\nu(D, V, \gamma)$ é igual ao tamanho máximo de um emparelhamento em $G$. É fácil ver que, neste caso, o corolário 7.2.1 é equivalente à fórmula 6.1.3 de Tutte-Berge: basta notar que, para qualquer $X \subseteq V$,

$$
\frac{1}{2}\left(|V|+|X|-q_{V}(G-X)\right)=|X|+\sum\left\{\left\lfloor\frac{1}{2}|K|\right\rfloor: K \in \mathcal{K}(G-X)\right\},
$$

onde $\mathcal{K}(H)$ denota o conjunto dos componentes do grafo $H$.

Vejamos agora uma das versões do teorema de Menger. Seja $S \subseteq V$ e $\{U, W\}$ uma partição de $S$. Um caminho de $G$ é um $(U, W)$-caminho se tem uma extremidade em $U$ e outra em $W$. Tome $\Gamma:=\mathbb{Z}_{2}$. Para cada arco $a$, atribua $\gamma_{a}:=1$ se $a$ possui exatamente uma ponta em $U$ e $\gamma_{a}:=0$ caso contrário. Então é evidente que um $S$-caminho é não-nulo se, e somente se, seu grafo subjacente é um $(U, W)$-caminho. Assim, o corolário 7.2.1 fornece uma relação min-max para o número máximo de $(U, W)$-caminhos disjuntos nos vértices. Note que uma das versões do teorema de Menger já fornecia uma relação min-max para esse problema.

Vamos ver agora que uma relação min-max de MADER [1978b] é um caso especial do corolário 7.2.1. Seja $S \subseteq V$ e $\mathcal{S}$ uma partição de $S$. Um caminho em $G$ é um $\mathcal{S}$-caminho se suas extremidades estão em partes distintas de $\mathcal{S}$. Mader provou a seguinte relação min-max:

\section{Corolário 7.2.2 (Teorema dos $\mathcal{S}$-caminhos disjuntos de Mader)}

Seja $G=(V, E)$ um grafo, $S \subseteq V$ e $\mathcal{S}$ uma partição de $S$. Então o número máximo de $\mathcal{S}$-caminhos disjuntos nos vértices é igual ao valor mínimo de

$$
|X|+\sum_{i=1}^{k}\left\lfloor\frac{1}{2}\left|\partial U_{i}\right|\right\rfloor,
$$

sendo que o mínimo é tomado sobre todas as partições $\left\{X, U_{1}, \ldots, U_{k}\right\}$ de $V$ tais que todo $\mathcal{S}$-caminho ou passa por $X$ ou então por alguma aresta induzida por algum $U_{i}$. A parte $X$ é considerada especial, pois pode ser vazia. Para cada $i$, denotamos por $\partial U_{i}$ o conjunto de vértices de $U_{i}$ que estão em $S$ ou que têm algum vizinho em $V \backslash\left(X \cup U_{i}\right)$.

Demonstração. Seja $D=(V, A)$ uma orientação arbitrária de $G$. Para $i=1,2$, seja $\Gamma_{i}$ um grupo e $\gamma^{i}: A \rightarrow \Gamma_{i}$ uma função peso. Defina o grupo $\Gamma:=\Gamma_{1} \times \Gamma_{2}$ da maneira usual e tome $\gamma_{a}:=\left(\gamma_{a}^{1}, \gamma_{a}^{2}\right)$ para cada $a \in A$. É evidente que um pseudocaminho é não-nulo com relação a $\gamma$ se, e somente se, é não-nulo com relação a $\gamma^{1}$ ou com relação a $\gamma^{2}$.

Para cada parte $T \in \mathcal{S}$, crie uma função peso $\gamma^{T}: A \rightarrow \mathbb{Z}_{2}$ tal que um $S$-caminho é não-nulo com relação a $\gamma^{T}$ se, e somente se, é um $(T, S \backslash T)$-caminho. Usando a construção do parágrafo anterior, é fácil criar uma função peso $\gamma: A \rightarrow \mathbb{Z}_{2}^{|\mathcal{S}|}$ tal que um $S$-caminho é não-nulo com relação a $\gamma$ se, e somente se, seu grafo subjacente é um $\mathcal{S}$-caminho.

Agora o resultado segue facilmente do corolário 7.2.1. 
O corolário 7.2.1 também fornece uma relação min-max para um problema resolvido por GALlai [1961], que descrevemos a seguir.

Seja $T \subseteq V$. Um caminho em $G$ é um $T$-caminho se suas extremidades são vértices distintos de $T$. Seja $\Gamma$ o grupo livre gerado por $\left\{\gamma_{a}: a \in A\right\}$. Então todo $T$-caminho em $G$ é um $T$-caminho não-nulo em $D$, e portanto o corolário 7.2.1 fornece uma relação min-max para o número máximo de $T$-caminhos disjuntos nos vértices. Uma relação min-max para esse problema já havia sido obtida por Gallai, usando a fórmula 6.1.3 de Tutte-Berge:

\section{Teorema 7.3 (Teorema dos $T$-caminhos disjuntos de Gallai)}

Seja $G=(V, E)$ um grafo e $T \subseteq V$. Então o número máximo de $T$-caminhos disjuntos nos vértices é igual a

$$
\min _{X \subseteq V}\left\{|X|+\sum\left\{\left\lfloor\frac{1}{2}|K \cap T|\right\rfloor: K \in \mathcal{K}(G-X)\right\}\right\}
$$

onde $\mathcal{K}(H)$ denota o conjunto dos componentes do grafo $H$.

O corolário 7.2.1 também tem como caso especial uma relação min-max para o número máximo de $T$-caminhos ímpares disjuntos nos vértices: basta tomar $\Gamma:=\mathbb{Z}_{2}$ e $\gamma_{a}:=1$ para todo $a \in A$.

\section{Resultados relacionados}

Chudnovsky, Cunningham e Geelen [2004] obtiveram um algoritmo polinomial para a relação min-max do corolário 7.2.1, onde cada operação de grupo é tratada como uma operação elementar. Esse é um algoritmo estrutural da mesma forma como o algoritmo de Lovász e Plummer [1986] e o algoritmo de SeBŐ [1986] mencionados nas notas no final do capítulo 6, ou seja, eles se baseiam num teorema estrutural do tipo Gallai-Edmonds. SEBŐ E SzEGŐ [2004] também obtiveram um teorema estrutural para o problema de $\mathcal{S}$-caminhos de Mader.

PAP [2005a, 2005b] obteve uma generalização da relação min-max do corolário 7.2.1, para a qual ele forneceu também um algoritmo polinomial.

O único outro algoritmo polinomial conhecido para o problema de $\mathcal{S}$-caminhos de Mader baseia-se numa complicada redução ao problema conhecido como linear matroid matching, para o qual Lovász obteve uma relação min-max e um algoritmo polinomial (veja os artigos de LovÁsz [1980b, 1980a, 1981]).

MADER [1978a, 1978b] também obteve relações min-max para o empacotamento máximo de $\mathcal{S}$-caminhos disjuntos nas arestas e internamente disjuntos. Este último caso é equivalente ao teorema 7.2.2 dos $\mathcal{S}$-caminhos disjuntos de Mader. Veja SchriJver [2003].

SCHRIJVER [2001] obteve uma prova curta do teorema 7.2.2 dos $\mathcal{S}$-caminhos disjuntos de Mader, reduzindo-o ao teorema 7.3 dos $T$-caminhos disjuntos de Gallai, que por sua vez é reduzido à fórmula 6.1.3 de Tutte-Berge.

Veja também Keijsper, Pendavingh e Stougie [2006] para uma formulação linear da versão disjunta nas arestas do problema de $\mathcal{S}$-caminhos de Mader. 


\section{Considerações finais}

Comprovamos nos capítulos anteriores que relações min-max são objetos fundamentais em otimização combinatória e fazem parte de uma rica e elegante teoria.

Apresentamos alguns teoremas bastante gerais, como por exemplo os arcabouços de fluxos submodulares e de $S$-caminhos não-nulos, e o poderoso teorema de Frank, Tardos e Sebö. Porém, e o que provavelmente é mais importante, vimos que existem várias técnicas de prova que foram usadas com sucesso para diversos problemas, como técnicas da combinatória poliédrica, o uso de funções submodulares, matróides, etc. Isso contrasta com o caráter ad hoc das provas originais das primeiras relações min-max, que dificilmente funcionariam para provar outros resultados.

Para ilustrar como as diversas relações min-max estão interligadas, exibimos na página seguinte um grafo orientado de relações min-max, onde um arco entre duas relações indica que a ponta final pode ser derivada a partir da ponta inicial. Arcos cheios indicam derivações presentes neste texto, enquanto arcos tracejados significam que a derivação pode ser encontrada na literatura, ou até mesmo obtida facilmente por alguma construção elementar. Relações min-max apresentadas no mesmo capítulo aparecem com a mesma cor. Próximo a cada vértice colocamos o número do teorema ou corolário a que o vértice se refere.

Pode-se constatar desse grafo que diversas derivações não foram incluídas neste texto. Claramente, mais arcos tracejados poderiam ser acrescentados ao grafo que exibimos. Não colocamos vários arcos implicados pela transitividade, e também não nos preocupamos em fazer um estudo exaustivo dessas inter-relações. O intuito aqui foi dar uma visão geral dos resultados e mostrar a abordagem que seguimos neste texto: provamos alguns poucos teoremas e a partir destes derivamos várias relações min-max. 


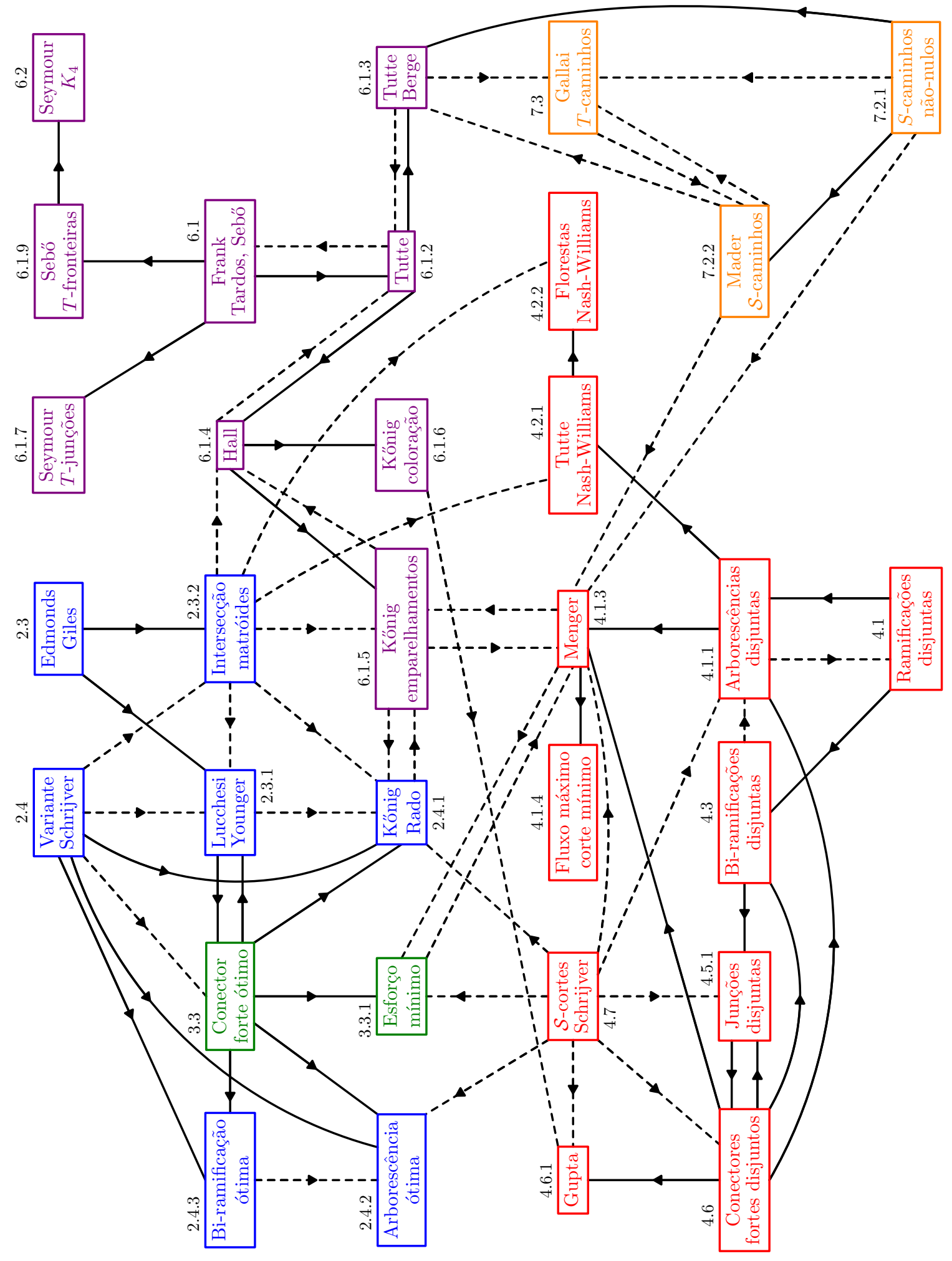




\section{Referências bibliográficas}

[1971] I. Anderson: Perfect matchings of a graph, Journal of Combinatorial Theory, Series $B 10$ (1971), pag. 183-186. [7, 105]

[1985] R. P. AnsteE: An algorithmic proof of Tutte's f-factor theorem, Journal of Algorithms 6 (1985), pag. 112-131. [104]

[1990] F. Barahona: Planar multicommodity flows, max cut, and the Chinese postman problem, in: Polyhedral Combinatorics (Proceedings DIMACS Workshop, Morristown, New Jersey, 1989; W. Cook, P. D. Seymour, editors), volume 1 de DIMACS Series in Discrete Mathematics and Theoretical Computer Science, American Mathematical Society, Providence, Rhode Island, 1990, pag. 189-202. [105]

[1994] F. Barahona, J. Fonlupt E A. R. Mahjoub: Compositions of graphs and polyhedra IV: Acyclic spanning subgraphs, SIAM Journal on Discrete Mathematics 7 (1994), pag. 390-402. [89]

[1958] C. Berge: Sur le couplage maximum d'un graphe, Comptes Rendus Hebdomadaires des Séances de l'Académie des Sciences [Paris] 247 (1958), pag. 258-259. [96]

[1971] F. Bock: An algorithm to construct a minimum directed spanning tree in a directed network, in: Developments in Operations Research, Volume 1 (Proceedings of the Third Annual Israel Conference on Operations Research, Tel Aviv, 1969; B. AviItzhak, editor), 1971, Gordon and Breach, New York, pag. 29-44. [32]

[1998] B. BollobÁs: Modern Graph Theory, volume 184 de Graduate Texts in Mathematics, Springer-Verlag, New York, 1998. [73]

[1995] A. Bouchet E W. H. Cunningham: Delta-matroids, jump systems, and bisubmodular polyhedra, SIAM Journal on Discrete Mathematics 8 (1995), pag. 17-32. [104]

[2004] M. Chudnovsky, W. H. Cunningham e J. Geelen: An algorithm for packing non-zero $A$-paths in group-labeled graphs. Submetido para publicação, 2004. [116]

[2006] M. Chudnovsky, J. Geelen, B. Gerards, L. Goddyn, M. Lohman e P. SeyMOUR: Packing non-zero $A$-paths in group-labeled graphs, Combinatorica 26 (2006), pag. 521-532. [105, 108, 114] 
[1996] P. Codato, M. Conforti e C. Serafini: Packing T-joins, Journal of Graph Theory 22 (1996), pag. 293-296. [105]

[1986] W. Cook, J. Fonlupt e A. Schrijver: An integer analogue of Carathéodory's theorem, Journal of Combinatorial Theory, Series B 40 (1986), pag. 63-70. [8, 19]

[2001] T. H. Cormen, C. E. Leiserson, R. L. Rivest e C. Stein: Introduction to Algorithms, second edition, MIT Press, Cambridge, Massachusetts, 2001. [9, 63]

[2002] G. Cornú́Jols E B. Guenin: On dijoins, Discrete Mathematics 243 (2002), pag. 213-216. [74, 75]

[2000] G. Cornuéjols, B. Guenin e F. Margot: The packing property, Mathematical Programming 89 (2000), pag. 113-126. [76]

[1984] W. H. Cunningham: Testing membership in matroid polyhedra, Journal of Combinatorial Theory, Series B 36 (1984), pag. 161-188. [34]

[1985] W. H. Cunningham: On submodular function minimization, Combinatorica 5 (1985), pag. 185-192. [34]

[2002] W. H. Cunningham: Matching, matroids, and extensions, Mathematical Programming 91 (2002), pag. 515-542. [104]

[1985] W. H. Cunningham e A. Frank: A primal-dual algorithm for submodular flows, Mathematics of Operations Research 10 (1985), pag. 251-262. [33]

[1997] W. H. Cunningham e J. F. Geelen: The optimal path-matching problem, Combinatorica 17 (1997), pag. 315-337. [104]

[1978] W. H. Cunningham E A. B. Marsh III: A primal algorithm for optimum matching, Mathematical Programming Study 8 (1978), pag. 50-72. Polyhedral Combinatorics - Dedicated to the Memory of D. R. Fulkerson (M. L. Balinski, A. J. Hoffman, editores). [103]

[1956] G. B. Dantzig E D. R. Fulkerson: On the max-flow min-cut theorem of networks, in: Linear Inequalities and Related Systems (H. W. Kuhn e A. W. Tucker, editores), Annals of Mathematics Studies 38, 1956, Princeton University Press, Princeton, New Jersey, pag. 215-221. [52]

[2005] R. Diestel: Graph Theory, third edition, volume 173 de Graduate Texts in Mathematics, Springer-Verlag, Berlin, 2005. [9, 14, 73, 81]

[1970] E. A. Dinits: Algoritm resheniya zadachi o maksimal'nom potoke v seti so stepennoŭ otsenkoŭ, Doklady Akademii Nauk SSSR 194 (1970), pag. 754-757. Em russo [Tradução para o inglês: Algorithm for solution of a problem of maximum flow in a network with power estimation]. [63] 
[2002] J. Donadelli e Y. Kohayakawa: A density result for random sparse oriented graphs and its relation to a conjecture of Woodall, Electronic Journal of Combinatorics 9 (2002). Research Paper 45, 10 pag. (electronic). [72, 73]

[1965a] J. Edmonds: The Chinese postman's problem, Bulletin of the Operations Research Society of America 13 (1965), pag. B-73. [105]

[1965b] J. Edmonds: Lehman's switching game and a theorem of Tutte and Nash-Williams, Journal of Research National Bureau of Standards Section B 69 (1965), pag. 73-77. $[34]$

[1965c] J. Edmonds: Maximum matching and a polyhedron with 0,1-vertices, Journal of Research National Bureau of Standards Section B 69 (1965), pag. 125-130. [103]

[1965d] J. EDmonds: Minimum partition of a matroid into independent subsets, Journal of Research National Bureau of Standards Section B 69 (1965), pag. 67-72. [55]

[1965e] J. Edmonds: Paths, trees, and flowers, Canadian Journal of Mathematics 17 (1965), pag. 449-467. [102, 103, 104]

[1967] J. Edmonds: Optimum branchings, Journal of Research National Bureau of Standards Section B 71 (1967), pag. 233-240. [32]

[1970] J. EDmonds: Submodular functions, matroids, and certain polyhedra, in: Combinatorial Structures and Their Applications (Proceedings Calgary International Conference on Combinatorial Structures and Their Applications, Calgary, Alberta, 1969; R. Guy, H. Hanani, N. Sauer e J. Schönheim, editores), 1970, Gordon and Breach, New York, pag. 69-87. [29]

[1973] J. Edmonds: Edge-disjoint branchings, in: Combinatorial Algorithms (Courant Computer Science Symposium 9, Monterey, California, 1972; R. Rustin, editor), 1973, Algorithmics Press, New York, pag. 91-96. [50]

[1977] J. Edmonds E R. Giles: A min-max relation for submodular functions on graphs, in: Studies in Integer Programming (Proceedings Workshop on Integer Programming, Bonn, 1975; P. L. Hammer, E. L. Johnson, B. H. Korte e G. L. Nemhauser, editores), volume 1 de Annals of Discrete Mathematics, 1977, North-Holland, Amsterdam, pag. 185-204. [18, 26, 27, 28, 66]

[1972] J. Edmonds E R. M. KARP: Theoretical improvements in algorithmic efficiency for network flow problems, Journal of the Association for Computing Machinery 19 (1972), pag. 248-264. [33, 63]

[1983] P. Feofiloff: Disjoint Transversals of Directed Coboundaries, tese de doutorado, Department of Combinatorics and Optimization, University of Waterloo, Waterloo, Ontario, 1983. [63] 
[1987] P. Feofiloff E D. H. Younger: Directed cut transversal packing for source-sink connected graphs, Combinatorica 7 (1987), pag. 255-263. [58, 62]

[1957] L. R. Ford JR. E D. R. Fulkerson: A simple algorithm for finding maximal network flows and an application to the Hitchcock problem, Canadian Journal of Mathematics 9 (1957), pag. 210-218. [63]

[1979a] A. Frank: Covering branchings, Acta Scientiarum Mathematicarum [Szeged] 41 (1979), pag. 77-81. [63]

[1979b] A. Frank: Kernel systems of directed graphs, Acta Scientiarum Mathematicarum [Szeged] $4 \mathbf{1}$ (1979), pag. 63-76. [30, 58]

[1980] A. Frank: On the orientation of graphs, Journal of Combinatorial Theory, Series B 28 (1980), pag. 251-261. [33]

[1981a] A. Frank: How to make a digraph strongly connected, Combinatorica 1 (1981), pag. $145-153 .[29,41]$

[1981b] A. Frank: On disjoint trees and arborescences, in: Algebraic Methods in Graph Theory, Vol. I (Colloquium Szeged, 1978; L. Lovász e V. T. Sós, editores), volume 25 de Colloquia Mathematica Societatis János Bolyai, North-Holland, Amsterdam, 1981, pag. 159-169. [63]

[1981c] A. Frank: A weighted matroid intersection algorithm, Journal of Algorithms 2 (1981), pag. 328-336. [47]

[1982] A. Frank: An algorithm for submodular functions on graphs, in: Bonn Workshop on Combinatorial Optimization (Bonn, 1980; A. Bachem, M. Grötschel e B. Korte, editores), volume 16 de Annals of Discrete Mathematics, North-Holland, Amsterdam, 1982, pag. 97-120. [29, 33, 41, 47]

[1984a] A. Frank: Finding feasible vectors of Edmonds-Giles polyhedra, Journal of Combinatorial Theory, Series B 36 (1984), pag. 221-239. [33]

[1984b] A. Frank: Generalized polymatroids, in: Finite and Infinite Sets, Vol. I (Proceedings Sixth Hungarian Combinatorial Colloquium, Eger, 1981; A. Hajnal, L. Lovász e V. T. Sós, editores), volume 37 de Colloquia Mathematica Societatis János Bolyai, North-Holland, Amsterdam, 1984, pag. 285-294. [30]

[1993] A. Frank: Submodular functions in graph theory, Discrete Mathematics 111 (1993), pag. 231-243. Graph Theory and Combinatorics (Marseille-Luminy, 1990; C. Berge, editor). [53]

[1996] A. Frank: A survey on $T$-joins, $T$-cuts, and conservative weightings, in: Combinatorics, Paul Erdős is Eighty, Volume 2 (D. Miklós, V. T. Sós, T. Szőni, editores), volume 2 de Bolyai Society Mathematical Studies, János Bolyai Mathematical Society, Budapest, 1996, pag. 213-252. [105] 
[1999a] A. Frank: Finding minimum generators of path systems, Journal of Combinatorial Theory, Series B $\mathbf{7 5}$ (1999), pag. 237-244. [34]

[1999b] A. Frank: Increasing the rooted-connectivity of a digraph by one, Mathematical Programming 84 (1999), pag. 565-576. [33]

[1995] A. Frank e T. Jordán: Minimal edge-coverings of pairs of sets, Journal of Combinatorial Theory, Series B 65 (1995), pag. 73-110. [34]

[2002] A. Frank E L. Szegö: Note on the path-matching formula, Journal of Graph Theory 41 (2002), pag. 110-119. [104]

[1994] A. Frank E Z. Szigeti: On packing T-cuts, Journal of Combinatorial Theory, Series B 61 (1994), pag. 263-271. [93, 98, 99]

[1984] A. Frank E É. Tardos: Matroids from crossing families, in: Finite and Infinite Sets, Vol. I (Proceedings Sixth Hungarian Combinatorial Colloquium, Eger, 1981; A. Hajnal, L. Lovász e V. T. Sós, editores), volume 37 de Colloq. Math. Soc. János Bolyai, North-Holland, Amsterdam, 1984, pag. 295-304. [47]

[1987] A. Frank E É. TARdos: An application of simultaneous diophantine approximation in combinatorial optimization, Combinatorica 7 (1987), pag. 49-65. [33]

[1988] A. Frank E É. TARdos: Generalized polymatroids and submodular flows, Mathematical Programming 42 (1988), pag. 489-563. [30]

[1989] A. FrAnk E É. TARdos: An application of submodular flows, Linear Algebra and Its Applications 114/115 (1989), pag. 329-348. [33]

[1984] A. Frank, É. Tardos E A. Sebő: Covering directed and odd cuts, Mathematical Programming Study 22 (1984), pag. 99-112. Mathematical Programming at Oberwolfach, II (Oberwolfach, 1983; B. Korte and K. Ritter, editores). [93]

[1987] J. Friedman E N. Pippenger: Expanding graphs contain all small trees, Combinatorica 7 (1987), pag. 71-76. [63]

[1986] S. FuJISHIGE: A capacity-rounding algorithm for the minimum-cost circulation problem: a dual framework of the Tardos algorithm, Mathematical Programming 35 (1986), pag. 298-308. [33]

[2005] S. FujISHIGE: Submodular Functions and Optimization, second edition, volume 58 de Annals of Discrete Mathematics, Elsevier, Amsterdam, 2005. [34]

[1989] S. Fujishige, H. Röck E U. Zimmermann: A strongly polynomial algorithm for minimum cost submodular flow problems, Mathematics of Operations Research 14 (1989), pag. 60-69. [33] 
[1971] D. R. FulKerson: Blocking and anti-blocking pairs of polyhedra, Mathematical Programming 1 (1971), pag. 168-194. [17]

[1974] D. R. Fulkerson: Packing rooted directed cuts in a weighted directed graph, Mathematical Programming 6 (1974), pag. 1-13. [32]

[1958a] T. Gallai: Gráfokkal kapcsolatos maximum-minimum tételek (II. rész), A Magyar Tudományos Akadémia Matematikai és Fizikai Tudományok Osztályának Közleményei 8 (1958), pag. 1-40. Em húngaro [Tradução para o inglês: Maximumminimum theorems for networks. (part II)]. [31]

[1958b] T. Gallai: Maximum-minimum Sätze über Graphen, Acta Mathematica Academiae Scientiarum Hungaricae 9 (1958), pag. 395-434. [31]

[1961] T. Gallai: Maximum-minimum Sätze und verallgemeinerte Faktoren von Graphen, Acta Mathematica Academiae Scientiarum Hungaricae 12 (1961), pag. 131-173. [112, $116]$

[1963] T. Gallai: Kritische Graphen II, A Magyar Tudományos Akadémia - Matematikai Kutató Intézetének Közleményei 8 (1963), pag. 373-395. [103]

[1964] T. Gallai: Maximale Systeme unabhängiger Kanten, A Magyar Tudományos Akadémia - Matematikai Kutató Intézetének Közleményei 9 (1964), pag. 401-413. [103]

[2000] J. F. GeELen: An algebraic matching algorithm, Combinatorica 20 (2000), pag. 6170. $[103]$

[2005] J. GeElen E S. Iwata: Matroid matching via mixed skew-symmetric matrices, Combinatorica 25 (2005), pag. 187-215. [105]

[2003] J. F. Geelen, S. Iwata e K. Murota: The linear delta-matroid parity problem, Journal of Combinatorial Theory, Series B 88 (2003), pag. 377-398. [105]

[1987] A. V. Goldberg: Efficient Graph Algorithms for Sequential and Parallel Computers, tese de doutorado, Laboratory for Computer Science, Massachusetts Institute of Technology, Cambridge, Massachusetts, 1987. Technical Report MIT/LCS/TR-374. $[63]$

[1988] A. V. Goldberg E R. E. TARJAn: A new approach to the maximum-flow problem, Journal of the Association for Computing Machinery 35 (1988), pag. 921-940. [63]

[1982] H. Gröflin E A. J. Hoffman: Lattice polyhedra II: Generalization, constructions and examples, in: Algebraic and Geometric Combinatorics (E. Mendelsohn, editor), volume 15 de Annals of Discrete Mathematics, North-Holland, Amsterdam, 1982, pag. 189-203. [30]

[1981] M. Grötschel, L. Lovász E A. SchriJver: The ellipsoid method and its consequences in combinatorial optimization, Combinatorica 1 (1981), pag. 169-197. [33] 
[1988] M. Grötschel, L. Lovász e A. SchriJver: Geometric Algorithms and Combinatorial Optimization, volume 2 de Algorithms and Combinatorics: Study and Research Texts, Springer-Verlag, Berlin, 1988. [33]

[1960] M.-G. GuAn: Graphic programming using odd or even points, Acta Mathematica Sinica 10 (1960), pag. 263-266. Em chinês. [92]

[2002] B. Guenin: A short proof of Seymour's characterization of the matroids with the max-flow min-cut property, Journal of Combinatorial Theory, Series B 86 (2002), pag. 273-279. [105]

[1967] R. P. Gupta: A decomposition theorem for bipartite graphs, in: Theory of Graphs - International Symposium - Théorie des graphes - Journées internationales d'étude (Rome, 1966; P. Rosenstiehl, editor), Gordon and Breach, New York, 1967, pag. 135-138. [63]

[1978] R. P. Gupta: An edge-coloration theorem for bipartite graphs with applications, Discrete Mathematics 23 (1978), pag. 229-233. [63]

[1984] E. GYŐRI: A minimax theorem on intervals, Journal of Combinatorial Theory, Series $B 37$ (1984), pag. 1-9. [34]

[1975] F. HADLOCK: Finding a maximum cut of a planar graph in polynomial time, SIAM Journal on Computing 4 (1975), pag. 221-225. [105]

[1935] P. Hall: On representatives of subsets, The Journal of the London Mathematical Society 10 (1935), pag. 26-30. [97]

[2006] N. J. A. HARVEY: Algebraic structures and algorithms for matching and matroid problems, in: Proceedings 47th Annual Symposium on Foundations of Computer Science (47th FOCS, Berkeley, California, 2006), 2006, IEEE Computer Society Press, Los Alamitos, California, pag. 531-542. [103]

[1982] R. Hassin: Minimum cost flow with set-constraints, Networks 12 (1982), pag. 1-21. [30]

[1976] A. J. Hoffman: On Lattice Polyhedra II: Construction and Examples. Report RC 6268, IBM Research Division, San Jose, California, 1976. [30]

[1978] A. J. Hoffman: On lattice polyhedra III: Blockers and anti-blockers of lattice clutters, Mathematical Programming Study (1978), pag. 197-207. Polyhedral combinatorics - Dedicated to the Memory of D. R. Fulkerson (M. L. Balinski e A. J. Hoffman, editores). [30]

[1978] A. J. Hoffman E D. E. Schwartz: On lattice polyhedra, in: Combinatorics Vol. I (Proceedings Fifth Hungarian Colloquium on Combinatorics, Keszthely, 1976; A. Hajnal e V. T. Sós, editores), volume 18 de Colloquia Mathematica Societatis János Bolyai, North-Holland, Amsterdam, 1978, pag. 593-598. [30] 
[2001] S. Iwata, L. Fleischer E S. Fujishige: A combinatorial strongly polynomial algorithm for minimizing submodular functions, Journal of the Association for Computing Machinery 48 (2001), pag. 761-777. [34]

[2005] S. Iwata, S. T. McCormick e M. Shigeno: A strongly polynomial cut canceling algorithm for minimum cost submodular flow, SIAM Journal on Discrete Mathematics 19 (2005), pag. 304-320 (electronic). [34]

[1975] E. L. Johnson: On cut-set integer polyhedra, Cahiers du Centre d'Études de Recherche Opérationnelle 17 (1975), pag. 235-251. [30]

[2006] J. C. M. Keijsper, R. A. Pendavingh e L. Stougie: A linear programming formulation of Mader's edge-disjoint paths problem, Journal of Combinatorial Theory, Series B 96 (2006), pag. 159-163. [116]

[1997] Y. Kohayakawa: Szemerédi's regularity lemma for sparse graphs, in: Foundations of Computational Mathematics (Rio de Janeiro, 1997; F. Cucker, M. Shub, editores), Springer-Verlag, Berlin, 1997, pag. 216-230. [73]

[1916] D. KöNIG: Graphok és alkalmazásuk a determinánsok és a halmazok elméletére, Mathematikai és Természettudományi Értesitő 34 (1916), pag. 104-119. Em húngaro. $[97]$

[1931] D. KöNIG: Graphok és matrixok, Matematikai és Fizikai Lapok 38 (1931), pag. 116119. Em húngaro [Tradução para o inglês: Graphs and matrices]. [97]

[1930] K. Kuratowski: Sur le problème des courbes gauches en topologie, Fundamenta Mathematicae 15 (1930), pag. 271-283. [13]

[1976] E. L. LAWLER: Combinatorial optimization: networks and matroids, Holt, Rinehart and Winston, New York, 1976. [105]

[1982a] E. L. LAwler E C. U. MARTel: Computing maximal "polymatroidal" network flows, Mathematics of Operations Research 7 (1982), pag. 334-347. [30, 33]

[1982b] E. L. LAWLER E C. U. MARTEL: Flow network formulations of polymatroid optimization problems, in: Bonn Workshop on Combinatorial Optimization (Bonn, 1980; A. Bachem, M. Grötschel e B. Korte, editores), volume 16 de Annals of Discrete Mathematics, North-Holland, Amsterdam, 1982, pag. 189-200. [30]

[2006] O. LeE: Comunicação pessoal, 2006. [88]

[2001] O. Lee E Y. WakABAyashi: Note on a min-max conjecture of Woodall, Journal of Graph Theory 38 (2001), pag. 36-41. [83]

[2006] O. Lee E A. Williams: Packing dicycle covers in planar graphs with no $K_{5}-e$ minor, in: Proceedings 7th Latin American Theoretical Informatics Symposium (7th LATIN, Valdivia, Chile, 2006), volume 3887 de Lecture Notes in Computer Science, Springer-Verlag, Berlin, 2006, pag. 677-688. [81, 84] 
[1982] A. K. Lenstra, H. W. Lenstra JR. E L. LovÁsz: Factoring polynomials with rational coefficients, Mathematische Annalen 261 (1982), pag. 515-534. [33]

[1975] L. LovÁsz: 2-matchings and 2-covers of hypergraphs, Acta Mathematica Academiae Scientiarum Hungaricae 26 (1975), pag. 433-444. [98]

[1976] L. Lovász: On two minimax theorems in graph, Journal of Combinatorial Theory, Series B 21 (1976), pag. 96-103. [34, 50, 63]

[1979] L. LovÁsz: On determinants, matchings, and random algorithms, in: Fundamentals of Computation Theory FCT 'r9 Proceedings of the Conference on Algebraic, Arithmetic, and Categorical Methods in Computation Theory (Berlin, 1979, L. Budach, editor), Akademie-Verlag, Berlin, 1979, pag. 565-574. [103]

[1980a] L. LovÁsz: Matroid matching and some applications, Journal of Combinatorial Theory, Series B 28 (1980), pag. 208-236. [105, 116]

[1980b] L. LovÁsz: Selecting independent lines from a family of lines in a space, Acta Scientiarum Mathematicarum [Szeged] 42 (1980), pag. 121-131. [105, 116]

[1981] L. LovÁsz: The matroid matching problem, in: Algebraic Methods in Graph Theory, Vol. II (Colloquium Szeged, 1978; L. Lovász, V. T. Sós, editores), volume 25 de Colloquia Mathematica Societatis János Bolyai, North-Holland, Amsterdam, 1981, pag. 495-517. [105, 116]

[1997] L. Lovász: The membership problem in jump systems, Journal of Combinatorial Theory, Series B $\mathbf{7 0}$ (1997), pag. 45-66. [104]

[1986] L. Lovász E M. D. Plummer: Matching Theory, volume 121 de North-Holland Mathematics Studies, North-Holland, Amsterdam, 1986. [102, 104, 116]

[1991] A. Lubiw: A weighted minimax relation for intervals, Journal of Combinatorial Theory, Series B 53 (1991), pag. 151-172. [34]

[1976] C. L. Lucchesi: A Minimax Equality for Directed Graphs, tese de doutorado, University of Waterloo, Ontario, 1976. [47]

[1978] C. L. Lucchesi E D. H. Younger: A minimax theorem for directed graphs, The Journal of the London Mathematical Society (2) 17 (1978), pag. 369-374. [29]

[1978a] W. Mader: Über die Maximalzahl kantendisjunkter A-Wege, Archiv der Mathematik (Basel) 30 (1978), pag. 325-336. [116]

[1978b] W. Mader: Über die Maximalzahl kreuzungsfreier $H$-Wege, Archiv der Mathematik (Basel) 31 (1978), pag. 387-402. [115, 116]

[1927] K. Menger: Zur allgemeinen Kurventheorie, Fundamenta Mathematicae 10 (1927), pag. $96-115$. [52] 
[2001] B. Mohar e C. Thomassen: Graphs on surfaces, Johns Hopkins Studies in the Mathematical Sciences, Johns Hopkins University Press, Baltimore, Maryland, 2001. $[9]$

[1960] C. St. J. A. Nash-Williams: On orientations, connectivity and odd-vertexpairings in finite graphs, Canadian Journal of Mathematics 12 (1960), pag. 555-567. $[33]$

[1961] C. St. J. A. Nash-Williams: Edge-disjoint spanning trees of finite graphs, The Journal of the London Mathematical Society 36 (1961), pag. 445-450. [54]

[1964] C. St. J. A. Nash-Williams: Decomposition of finite graphs into forests, The Journal of the London Mathematical Society 39 (1964), pag. 12. [55]

[1981] H. Okamura e P. D. Seymour: Multicommodity flows in planar graphs, Journal of Combinatorial Theory, Series B 31 (1981), pag. 75-81. [63]

[1992] J. G. OxLEY: Matroid theory, Oxford Science Publications, Oxford University Press, Oxford, 1992. [9]

[1982] M. W. Padberg e M. R. RaO: Odd minimum cut-sets and b-matchings, Mathematics of Operations Research 7 (1982), pag. 67-80. [105]

[2004] G. PAP: Alternating paths revisited I: even factors, Relatório técnico TR-2004-18, Egerváry Research Group, Budapest, 2004. [104]

[2005a] G. PAP: Packing non-returning A-paths, Relatório técnico TR-2005-12, Egerváry Research Group, Budapest, 2005. [116]

[2005b] G. PAP: Packing non-returning A-paths algorithmically, in: Proceedings 2005 European Conference on Combinatorics, Graph Theory and Applications (EuroComb 2005, Berlin; S. Felsner, editor), S. Felsner, editor, volume AE de DMTCS Proceedings, Discrete Mathematics and Theoretical Computer Science, 2005, pag. 139-144. $[116]$

[2004] G. PAP E L. Szegö: On the maximum even factor in weakly symmetric graphs, Journal of Combinatorial Theory, Series B 91 (2004), pag. 201-213. [104]

[1933] R. RADO: Bemerkungen zur Kombinatorik im Anschluß an Untersuchungen von Herrn D. König, Sitzungsberichte der Berliner Mathematischen Gesellschaft 32 (1933), pag. 60-75. [31]

[1999] M. L. REY: T-junções, T-cortes e funções conservativas, dissertação de mestrado, Universidade de São Paulo, São Paulo, Brasil, 1999. [105]

[1998] R. RIzzI: Kőnig's edge coloring theorem without augmenting paths, Journal of Graph Theory 29 (1998), pag. 87. [102] 
[2000] R. Rizzi: A short proof of König's matching theorem, Journal of Graph Theory 33 (2000), pag. 138-139. [102]

[1939] H. E. RobBins: A theorem on graphs, with an application to a problem of traffic control, The American Mathematical Monthly 46 (1939), pag. 281-283. [71]

[1995] N. Robertson, P. D. Seymour e R. Thomas: Sachs' linkless embedding conjecture, Journal of Combinatorial Theory, Series B 64 (1995), pag. 185-227. [89]

[1980] H. Röck: Scaling techniques for minimal cost network flows, in: Discrete Structures and Algorithms (Proceedings Workshop WG79 5th Conference on Graph-theoretic Concepts in Computer Science, 1979; U. Pape, editor), Carl Hanser, München, 1980, pag. 181-191. [33]

[1980] P. Schönsleben: Ganzzahlige Polymatroid-Intersektions-Algorithmen, tese de doutorado, Eidgenössische Technische Hochschule Zürich, 1980. [33]

[1980] A. SchriJver: A counterexample to a conjecture of Edmonds and Giles, Discrete Mathematics 32 (1980), pag. 213-215. [66]

[1982] A. SchriJver: Min-max relations for directed graphs, in: Bonn Workshop on Combinatorial Optimization (Bonn, 1980; A. Bachem, M. Grötschel, B. Korte, editores), volume 16 de Annals of Discrete Mathematics, North-Holland, Amsterdam, 1982, pag. 261-280. [30, 32, 45, 56, 58, 62, 70]

[1983a] A. SchriJver: Min-max results in combinatorial optimization, in: Mathematical Programming - The State of the Art (Bonn, 1982; A. Bachem, M. Grötschel, B. Korte, editores), Springer, Berlin, 1983, pag. 439-500. [103]

[1983b] A. SchriJver: Packing and covering of crossing families of cuts, Journal of Combinatorial Theory, Series B 35 (1983), pag. 104-128. [64]

[1984a] A. SchriJVER: Proving total dual integrality with cross-free families - a general framework, Mathematical Programming 29 (1984), pag. 15-27. [30]

[1984b] A. SchriJver: Total dual integrality from directed graphs, crossing families, and sub- and supermodular functions, in: Progress in Combinatorial Optimization (Proceedings Conference, Waterloo, Ontario, 1982; W. R. Pulleyblank, editor), Academic Press, Toronto, Ontario, 1984, pag. 315-361. [30]

[1985] A. SchriJver: Supermodular colourings, in: Matroid Theory (Proceedings Colloquium on Matroid Theory, Szeged, 1982; L. Lovász, A. Recski, editores), volume 40 de Colloquia Mathematica Societatis János Bolyai, North-Holland, Amsterdam, 1985, pag. 327-343. [64]

[1986] A. SchriJver: Theory of Linear and Integer Programming, Wiley, Chichester, 1986. $[9,19,33]$ 
[2000] A. SchriJver: A combinatorial algorithm minimizing submodular functions in strongly polynomial time, Journal of Combinatorial Theory, Series B 80 (2000), pag. 346-355. [33]

[2001] A. SCHRIJVER: A short proof of Mader's $\mathscr{S}$-paths theorem, Journal of Combinatorial Theory, Series B $\mathbf{8 2}$ (2001), pag. 319-321. [116]

[2003] A. SchriJver: Combinatorial Optimization. Polyhedra and Efficiency, volume 24 de Algorithms and Combinatorics, Springer-Verlag, Berlin, 2003. [9, 47, 55, 59, 63, $71,77,80,102,116]$

[1986] A. SEBŐ: Finding the $t$-join structure of graphs, Mathematical Programming 36 (1986), pag. 123-134. [105, 116]

[1987] A. SЕвŐ: A quick proof of Seymour's theorem on $t$-joins, Discrete Mathematics 64 (1987), pag. 101-103. [93]

[1988] A. SEBö: The Schrijver system of odd join polyhedra, Combinatorica 8 (1988), pag. 103-116. [98, 99]

[1990] A. SEBö: Undirected distances and the postman-structure of graphs, Journal of Combinatorial Theory, Series B 49 (1990), pag. 10-39. [105]

[2004] A. Sebő E L. Szegő: The path-packing structure of graphs, in: Integer Programming and Combinatorial Optimization (Proceedings 10th IPCO Conference, New York, 2004; D. Bienstock, G. Nemhauser, editores), volume 3064 de Lecture Notes in Computer Science, Springer, Berlin, 2004, pag. 256-270. [104, 116]

[1977] P. D. Seymour: The matroids with the max-flow min-cut property, Journal of Combinatorial Theory, Series B 23 (1977), pag. 189-222. [99, 102, 105]

[1980] P. D. Seymour: Decomposition of regular matroids, Journal of Combinatorial Theory, Series B 28 (1980), pag. 305-359. [33]

[1981] P. D. Seymour: On odd cuts and plane multicommodity flows, Proceedings of the London Mathematical Society. Third Series 42 (1981), pag. 178-192. [98, 105]

[1996] P. D. Seymour: Packing circuits in Eulerian digraphs, Combinatorica 16 (1996), pag. 223-231. [89]

[1993] P. D. Seymour E R. Thomas: Graph searching and a min-max theorem for treewidth, Journal of Combinatorial Theory, Series B 58 (1993), pag. 22-33. [8]

[2005] F. B. Shepherd E A. Vetta: Visualizing, finding and packing dijoins, in: Graph Theory and Combinatorial Optimization, volume 8 de GERAD 25th Anniv. Ser., Springer-Verlag, New York, 2005, pag. 219-254. [77] 
[2004] B. Spille E L. Szegő: A Gallai-Edmonds-type structure theorem for pathmatchings, Journal of Graph Theory 46 (2004), pag. 93-102. [104]

[2002] B. Spille E R. Weismantel: A generalization of Edmonds' matching and matroid intersection algorithms, in: Integer Programming and Combinatorial Optimization (Proceedings 9th IPCO Conference, Cambridge, Massachusetts, 2002; W. Cook, A. S. Schulz, editores), volume 2337 de Lecture Notes in Computer Science, Springer, Berlin, 2002, pag. 9-20. [104]

[1978] E. SzemerÉd: Regular partitions of graphs, in: Problèmes Combinatoires et Théorie des Graphes (Colloques Internationaux, CNRS, Univ. Orsay, Orsay, 1976), CNRS, Paris, 1978, pag. 399-401. [73]

[2004] Z. Szigeti: On a min-max theorem on bipartite graphs, Discrete Mathematics 276 (2004), pag. 353-361. [7, 105]

[1985] É. TARDos: A strongly polynomial minimum cost circulation algorithm, Combinatorica 5 (1985), pag. 247-255. [33]

[1980] C. Thomassen: Planarity and duality of finite and infinite graphs, Journal of Combinatorial Theory, Series B 29 (1980), pag. 244-271. [81]

[1992] K. Truemper: Matroid Decomposition, Academic Press, San Diego, California, 1992. [33]

[1947] W. T. TutTE: The factorization of linear graphs, The Journal of the London Mathematical Society 22 (1947), pag. 107-111. [96, 103]

[1952] W. T. TutTE: The factors of graphs, Canadian Journal of Mathematics 4 (1952), pag. 314-328. [104]

[1961a] W. T. TUTTE: On the problem of decomposing a graph into $n$ connected factors, The Journal of the London Mathematical Society 36 (1961), pag. 221-230. [54]

[1961b] W. T. TutTe: A theory of 3-connected graphs, Indagationes Mathematicae [= Proceedings of the Koninklijke Nederlandse Akademie van Wetenschappen] 23 (1961), pag. 441-455. [81]

[1965] W. T. TutTE: Lectures on matroids, Journal of Research National Bureau of Standards Section B 69 (1965), pag. 1-47. [24]

[1974] W. T. TutTe: Spanning subgraphs with specified valencies, Discrete Mathematics 9 (1974), pag. 97-108. [104]

[1981] W. T. TutTe: Graph factors, Combinatorica 1 (1981), pag. 79-97. [104]

[1937a] K. WaGneR: Über eine Eigenschaft der ebenen Komplexe, Mathematische Annalen 114 (1937), pag. 570-590. [13] 
[1937b] K. Wagner: Über eine Erweiterung eines Satzes von Kuratowski, Deutsche Mathematik 2 (1937), pag. 280-285. [89]

[1960] K. Wagner: Bemerkungen zu Hadwigers Vermutung, Mathematische Annalen 141 (1960), pag. 433-451. [81]

[1976] D. J. A. Welsh: Matroid theory, Academic Press, London, 1976. [9]

[1932] H. Whitney: Congruent graphs and the connectivity of graphs, American Journal of Mathematics 54 (1932), pag. 150-168. [14]

[2004] A. M. Williams: Packing directed joins, dissertação de mestrado, University of Waterloo, Ontario, 2004. [75, 76]

[1978] D. R. Woodall: Menger and König systems, in: Theory and Applications of Graphs (Proceedings Third International Conference Kalamazoo, Michigan, 1976; Y. Alavi, D. R. Lick, editores), volume 642 de Lecture Notes in Mathematics, Springer, Berlin, 1978, pag. 620-635. [69] 


\section{Índice remissivo}

$\geq 0,10$

0, 17

1, 17

$\infty, 17$

$a^{*}, 16$

$A_{0}(S, \gamma), 111$

$A(D), 14$

$A_{D}, 14$

algoritmo

caminhos aumentadores, 63

corte orientado de capacidade mínima, 69

de Frank, 29, 38, 41

de Lucchesi, 47

empacotamento máximo de

$S$-caminhos não-nulos, 116

emparelhamento

de peso máximo, 103

emparelhamento máximo, 102

estrutural, 104, 105, 116

estrutural de Chudnovsky, Cunningham e

Geelen, 116

estrutural de Lovász e Plummer, 104

estrutural de Sebő, 105

fluxo submodular de peso máximo, 29, $33,35,41,47$

intersecção de matróides com pesos, 47

junção de custo mínimo, 44

minimização de funções

submodulares, 33

primal-dual, 35, 38, 41, 103

push-relabel, 63

$(r, s)$-corte de capacidade mínima, 52, 63,

69
T-corte de capacidade mínima, 105

Anderson, 7, 105

Anstee, 104

aproximação diofantina simultânea, 33

arborescência, 16

arcabouço, 23, 27, 30, 32, 35, 45, 49, 62, 64, 107

$\operatorname{arco}$

artificial, 39, 53

contração, 15

crítico, 85

de transferência, 68

direto, 15

entra em $S, 15$

incide, 14

incidente, 14

novo, 10

pontas, 14

ponta final, 14

ponta inicial, 14

reverso, 15

sai de $S, 15$

transitivo, 15, 69

vai de $u$ a $v, 14$

arcos, 14

correspondentes, 68

induzidos, 14

paralelos, 14

aresta

contração, 13

incide, 11

incidente, 11

liga $u$ e $v, 11$

negativa, 94 
nova, 10

pontas, 11

subdivisão, 95

arestas, 11

paralelas, 12

árvore, 12

geradora, 12

orientada, 14

$A_{D}[S], 14$

Barahona, 89, 105

base, 20

de $F, 21$

Berge, 96, 103, 104, 115, 116

blossoms, 102

Bock, 32

Bollobás, 73

Bouchet, 104

$B(\mathcal{P}), 109$

caminho, 12, 15

aumentador, 33

comprimento, 12

de aumento, 102, 104

extremidades, 12

liga $u$ a $v, 12$

vai de $u$ a $v, 12$

capacidade, 10

$\chi^{S}, 10$

Chudnovsky, 105, 107, 108, 114, 116

circuito, 12, 15, 22

axial, 40

comprimento, 12

de custo negativo, 44

ímpar, 12, 102

par, 12

clutter, 16

bloqueador, 16

co-ramificação, 56

cobertura

para $\mathcal{S}, 64$

por arestas, 31, 63

por vértices, 97 cobertura bisupermodulra, 34

Codato, 105

colagem, 33, 56

coleção

disjunta, 10

fechada sob cruzamentos, 27

coloração de arestas, 97

colorações supermodulares, 64

combinatória poliédrica, 4

componente, 12

forte, 15

fraco, 15

conector, 49

forte, $32,45,62$

Conforti, 105

conjectura

Edmonds-Giles, 3, 6, 7, 58, 65-77, 81

planar de Edmonds-Giles, 72, 73, 81

planar de Woodall, 72, 73

Woodall, 3, 7, 65, 69-72

conjunto

compatível, 92

contração, 13, 15

estável, 31

$S$-balanceado, 108

T-ímpar, 93

$T$-par, 93

tamanho, 10

conjuntos

diferença simétrica, 10

que se cruzam, 25

contra-exemplo

de Cornuéjols e Guenin, 74, 75

de Schrijver, 66, 72, 75

de Thomassen, 72

família infinita de Schrijver, 67

contração, 13, 15

Cook, 8, 19

Cormen, 9, 63

Cornuéjols, 65, 74-76

corte, 49

enraizado, 32

induzido por $\mathcal{S}, 64$ 
orientado, 16

$c_{p}(u v), 38$

cruzamento, 25

$\mathcal{C}_{T, \pi}, 25$

Cunningham, 33, 34, 103, 104, 116

custo, 10

reduzido, 38

cut-set polyhedra, 30

$D^{*}, 16$

$D-B, 15$

$D-S, 14$

$D / S, 15$

$D_{0}$-corte, 45,62

Dantzig, 52

decomposição, 33, 81, 89

em orelhas, 70

$\operatorname{def}(D, S, \gamma), 109$

deficiência, 109

delta-matróide, 105

$\Delta(G), 12$

$\delta(G), 12$

$\vec{\delta}(\mathcal{N}), 36$

$\delta(\mathcal{P}), 53$

$\delta_{F}(S), 12$

$\delta_{G}(S), 12$

$\vec{\delta}_{B}(S), 15$

$\overrightarrow{\delta_{D}}(S), 15$

$\overleftarrow{\delta}_{D}(S), 15$

$\overleftarrow{\delta}_{B}(S), 15$

descruzamento, $5,23,34$

deslocamento, 83, 108

$\operatorname{det}(M), 11$

diagrama de Venn, 25

Diestel, 9, 14, 73, 81

Dinits, 63

distributive lattices, 30

$D^{\prime}(J, p), 42$

$D(J, p), 39$

Donadelli, 72, 73

$D[S], 14$

$\vec{d}_{B}(S), 15$

$\overleftarrow{d}_{B}(S), 15$
$d_{F}(S), 12$

$d_{G}(S), 12$

dual, $72,81,88$

dualidade no plano, $72,81,88$

Edmonds, 2, 3, 5-7, 18, 23, 26-34, 45, 49, 50, 52-59, 62, 63, 65-77, 81, 91, 102-105, 116

$E(G), 11$

$E_{G}, 11$

$\mathcal{E}_{\mathcal{H}}, 16$

eliminação gaussiana, 103

elipsóides, 33

empacotamento, 49

de arborescências, 63

de árvores geradoras, 53

de cortes orientados, 35

de junções, 65

de $T$-cortes, 91

empacotamento fracionário, 76

semi-integral, 76

valor, 76

emparelhamento, 13

extensão, 104, 107

perfeito, 96

quase-perfeito, 103

satura $S, 96$

satura $v, 96$

envelope, 81, 88

$E_{G}[S], 12$

extensão transitiva, 70

$f$-fator, 104

família, 11

bidisjunta, 98

c-disjunta, 11

$f$-disjunta, 11

laminar, 25

livre de cruzamentos, 25, 28

representação arbórea, 25, 26, 30

representação arbórea enraizada, 25

tamanho, 11

w-disjunta, 11 
Feofiloff, 49, 58, 62, 63, 65, 70, 76, 80, 88

Fleischer, 33

floresta, 12

fluxo submodular, 27, 35

Fonlupt, 8, 19, 89

fonte, 15

Ford, 63

fórmula de Tutte-Berge, 96, 103, 104, 115, 116

Frank, 3, 7, 29, 30, 33-35, 38, 39, 41, 44, 45, $47,53,58,63,71,78,91,93,95,98$, 99, 104, 105, 117

Friedman, 63

Fujishige, 33, 34

Fulkerson, 17, 32, 47, 52, 63

função

capacidade, 10

conservativa, 92, 105

custo, 10

peso, 10

submodular, 10

submodular em cruzamentos, 27

supermodular, 10

supermodular em cruzamentos, 30

função peso $S$-equivalente, 108

função submodular minimização, 33,45

$G^{*}, 13$

$G-F, 12$

$G-S, 12$

$G / S, 13$

Gallai, 31, 103-105, 112, 116

$\gamma(a, v), 108$

Geelen, 103-105, 107, 108, 114, 116

generalized polymatroids, 30

Gerards, 105, 107, 108, 114

G/F, 13

Giles, 3, 6, 7, 18, 23, 26-31, 33, 58, 65-77, 81

Goddyn, 105, 107, 108, 114

Goldberg, 63

grafo, 11

acíclico, 12

bicrítico, 100 bipartição, 13

bipartido, 13

completo, 13

conexo, 12

conjunto de arestas, 11

conjunto de vértices, 11

cúbico, 12

densidade, 12

de fronteiras, 100

floresta, 12

grau máximo, 12

grau mínimo, 12

isomorfo, 13

$k$-aresta-conexo, 12

$k$-conexo, 12

$k$-regular, 12

linklessly embeddable, 89

livre de $H$ como menor, 13

matriz de incidência, 13, 24

menor, 13

orientação, 14

planar, 13

regular, 12

simples, 12

sobre $V, 11$

subgrafo, 12

subgrafo gerador, 12

subgrafo induzido, 12

subjacente, 14

supergrafo, 12

(U,W)-bipartido, 13

grafo bipartido

classes de cor, 13

grafo orientado, 14

acíclico, 15

conjunto de arcos, 14

conjunto de vértices, 14

densidade, 14

euleriano, 89

fonte-sorvedouro-conexo, 15

fortemente conexo, 15

fracamente conexo, 15

grafo subjacente, 14 
livre de $H$ como menor, 14

matriz de incidência, 16, 24

menor, 14

planar, 16

simples, 14

subgrafo, 14

grafo orientado planar

dual, 16

grafo planar

dual, 13

face, 13

graft, 101

menor, 101

Gröflin, 30

Grötschel, 33

grupo, 108

abeliano, 108

livre, 116

$G[S], 12$

Guan, 92, 93

Guenin, 65, 74-76, 105

$G \cup H, 13$

Gupta, 63

$g_{F}(v), 12$

$g_{G}(v), 12$

$G \times H, 13$

Győri, 34

Hadlock, 105

Hall, 7, 97, 105

Harvey, 103

Hassin, 30

hiper-arestas, 16

hipergrafo, 16, 37

componente, 16

conexo, 16

conjunto de hiper-arestas, 16

sobre $V, 16$

hipo-emparelhável, 103

Hoffman, 30

$\infty, 17$

Iwata, 33, 34, 105
Johnson, 30

Jordán, 34

jump system, 104, 105

junção, 16 adequada, 70 admissível, 70

admissível, 78

redutora, 78

$k$-junção, 59

$K_{3,3}, 13,72,89$

$K_{4}, 83$

$K_{5}, 13,89$

$K_{5}-e, 81$

Karp, 33, 63

Keijsper, 116

kernel systems, 30

$K_{m, n}, 13$

$\mathcal{K}(N), 37$

$K_{n}, 13$

Kohayakawa, 72, 73

König, 2, 8, 31, 47, 91, 97, 102

$\kappa(S), 36$

Kuratowski, 13

$K_{U, W}, 13$

$K_{V}, 13$

laço, 12, 14, 22

lattice polyhedra, 30

Lawler, 30, 33, 105

Lee, $6,65,81,83,84,88$

Leiserson, 9, 63

lema

descruzamento, 27, 28, 31

forte da remoção, 84, 86

fraco da remoção, 84, 85

Guan, 92, 93

regularidade, 73

Lenstra, 33

$\mathcal{L}(N), 36$

Lohman, 105, 107, 108, 114

Lovász, 33, 34, 50, 63, 98, 102-105, 116

Lubiw, 34 
Lucchesi, 3, 8, 23, 27, 29, 32-35, 44-47, 58, NP-difícil, 45, 69, 105

$62,65,66,72,76,78,89$

Mader, 107, 115, 116

Mahjoub, 89

Margot, 76

Marsh, 103

Martel, 30, 33

matriz

anti-simétrica, 103

de rede, 33

determinante, 11

de incidência, 13, 16, 24

de rede, 24,70

de Tutte, 103, 105

sobre $U \times W, 10$

totalmente unimodular, $11,24,26,28,30$, $31,33,70,91$

matróide, 5, 20, 29, 33, 91, 99, 110

base, 20

circuito, 22

conjunto-base, 20

conjunto dependente, 20

conjunto independente, 20

elementos paralelos, 22

função posto, 21, 29

laço, 22

linear, 105

sobre $E, 20$

matroid matching, 105, 116, 139

matroid parity, 105, 139

McCormick, 34

Menger, 52, 62, 115

menor, 13, 14, 101

proibido, 5, 13, 81, 83, 84, 88, 89, 102

método dos elipsóides, 33

Mohar, 9

multiplicidade, 11

Murota, 105

Nash-Williams, 5, 33, 49, 53-55

$N_{\min }(v), 37,44$

novo, 10
$N_{G}(S), 11$

núcleo, 36

fechado, 37

folga, 40

justo, 36

$N_{\min }(v), 37,44$

partição justa, 37

perigoso, 36

$\nu(D, S, \gamma), 108$

$\nu(D, w), 81$

Okamura, 63

operação de redução, 75

orelha, 70

Oxley, 9

Padberg, 105

Pap, 104, 116

par, 10

parte, 10

partição, 10

path-matching, 104

Pendavingh, 116

peso, 10

Pippenger, 63

Plummer, 102, 104, 116

polar, 49, 65

poliedro, 17

integral, 17

vértice, 17

politopo dos emparelhamentos, 103

polymatroidal network flows, 30

posto de $F, 21$

potencial, 38

pré-ordem, 80

prisma, 81

triangular, 81

problema

aumento de conexidade, 27, 34

aumento de conexidade enraizada, 33

caminho de custo mínimo, 105

chinês do carteiro, 91, 105 
circulação de custo mínimo, 27, 33, 44

conector forte de custo mínimo, 45

corte máximo, 91, 105

empacotamento máximo de cortes orientados, 35

empacotamento máximo de junções, 69

emparelhamento de peso máximo, 103

emparelhamento máximo, 102

emparelhamento perfeito

de peso mínimo, 105

fluxo submodular de peso máximo, 29, $33,35,41,47$

junção de custo mínimo, 35

linear matroid matching, 105, 116

matroid matching, 105, 116

matroid parity, 105

multifluxos, 91, 105

orientação, 27, 33

programação linear, 17

$(r, s)$-corte mínimo, 49, 63

$\mathcal{S}$-caminhos de Mader, 107, 116

$T$-junção de peso mínimo, 105

programação linear, 17

programa linear, 17

dual, 17

primal, 17

solução ótima, 17

vetor viável, 17

propriedade

de troca, 5, 20, 56, 57

fluxo-máximo corte-mínimo, 91, 99

pseudocaminho, 15

arco direto, 15

arco reverso, 15

comprimento, 15

extremidade, 15

extremidade final, 15

extremidade inicial, 15

liga $u$ a $v, 15$

não-nulo, 108

peso, 108

solto, 109

vai de $u$ a $v, 15$ pseudocaminhos

consecutivos, 68

do tipo 1 ou 2,68

pseudocircuito, 15

arco direto, 15

arco reverso, 15

comprimento, 15

não-nulo, 108

peso, 108

$\mathcal{P}(V), 10$

$\mathbb{Q}, 10$

$q_{T}(H), 93$

quebra-circuitos, 72

adequado, 83

admissível, 83

$\mathbb{R}, 10$

$r$-arborescência, 16, 32

$R$-co-ramificação, 56

$r$-corte, 32,52

$R$-ramificação, 16

Rado, 31, 47

ramificação, 16

conjunto de raízes, 16

raiz, 16

Rao, 105

representação arbórea, 25, 26, 30 enraizada, 25

reticulado, 80

Rey, 105

$\varrho^{*}(w, U), 83$

$\varrho(w, U), 83$

Rivest, 9, 63

Rizzi, 102

Robbins, 71

Robertson, 89

Röck, 33

roda, 81

Rödl, 73

$(R, S)$-bicorte, 32,56

$(R, S)$-bi-ramificação, 32,56

$(r, s)$-caminho, 15 
$(r, s)$-corte, 15

$\mathbb{R}^{V}, 10$

$S$-caminho, 108

$\mathcal{S}$-caminho, 115

$S$-coleção, 109

ótima, 109

$S_{a}, 25$

scaling, 33

Schönsleben, 33

Schrijver, 3, 5, 8, 9, 19, 23, 30-33, 35, 45-47, $49,55,56,58-68,70-72,74-77,80$,

Schwartz, 30 $88,102,103,107,116$

Sebő, 7, 91-93, 95, 98, 99, 101, 104, 105, 116, 117

Serafini, 105

Seymour, 6, 8, 33, 63, 89, 91, 98, 99, 102, 105, 107, 108, 114

Shepherd, 77

Shigeno, 34

sistema de inequações lineares, 17

box-TDI, 18, 28, 31

totalmente dual integral, 18

sorvedouro, 15

Spille, 104

Stein, 9, 63

Stougie, 116

subdivisão, 95

subgrafo, 12, 14

gerador, 12

induzido, 12, 14

T-ímpar, 93

$T$-par, 93

submodularidade, 4, 10, 23, 34, 36, 51, 55

super-fonte, 76

super-sorvedouro, 76

supergrafo, 12

supermodularidade, 10

Szegö, 104, 116

Szemerédi, 73

Szigeti, 7, 91, 93, 98, 99, 105

T-caminho, 116
$T$-corte, 93

$T$-fronteira, 98

bicrítica, 100

valor, 98

T-junção, 92 mínima, 92

$T$-partição, 98

valor, 98

tamanho, 10

Tardos, 7, 30, 33, 47, 91, 93, 95, 98, 99, 105, 117

Tarjan, 63

$\tau(D, w), 81$

teorema

arborescências disjuntas de

Edmonds, 52-54, 58, 59, 62, 63

arborescência ótima de Fulkerson, 32, 47

árvores geradoras disjuntas de Tutte e

Nash-Williams, 5, 49, 54, 55

bi-ramificação ótima de Schrijver, 32, 47, 56

bi-ramificações disjuntas de Schrijver, 5, $49,56,58-61,63,64$

capacitado de Lucchesi-Younger, 44-47,

$58,62,78$

casamento de Hall, 7, 97, 105

cobertura por arestas de Konnig-Rado, 31, 47

coloração de arestas de König, 97, 102

conectores fortes disjuntos de Schrijver,

$49,62-64$

conector forte ótimo de Schrijver, 32, 46, 47,62

decomposição, 33, 81, 89

Edmonds-Giles, 23, 28-31, 33

emparelhamentos de Kőnig, 91, 97, 102

emparelhamentos de König, 97

emparelhamentos perfeitos de Tutte, 96,

97, 100, 105

estrutural de Gallai-Edmonds, 103, 104

estrutural do tipo

Gallai-Edmonds, 104, 105, 116

fluxo máximo e corte mínimo, 52 
Frank, Tardos e Sebő, 7, 91, 93, 95, 98, 99, 105, 117

Frank, Tardos e Sebő, 93

intersecção de matróides de Edmonds, 23, $29,32,33,45,104$

intersecção de polimatróides de

Edmonds, 27, 29

junções disjuntas de Feofiloff-Younger e Schrijver, 62, 63, 70, 76, 80, 88

Lee e Williams, 6, 81, 84, 88

Lucchesi-Younger, 3, 8, 23, 27, 29, 32-35, $44,47,65,66,72,76$

Menger, 52, 62, 115

orientação de Nash-Williams, 33

partição em florestas de Nash-Williams, 49,55

planar de Lucchesi-Younger, 72, 89

potencial máximo e esforço mínimo, 47

ramificações disjuntas de Edmonds, 5, 49, $50,52,56,57,63$

$\mathcal{S}$-caminhos disjuntos de Mader, 107, 115, 116

T-caminhos disjuntos de Gallai, 116

Thomas, 8, 89

Thomassen, 9, 72, 81

torneio, 14

total dual integralidade, 18

tree projection, 33

tripla crítica, 111

Truemper, 33

Tutte, 5, 24, 49, 53-55, 81, 91, 96, 97, 100, 103-105, 115, 116

$\mathbf{1}, 17$

uncrossing, 23

unimodularidade total, 11, 24, 26, 28, 30, 31, $\mathbb{Z}^{V}, 10$ $33,70,91$

$\bigcup \mathcal{S}, 10$

variante de Schrijver, 31, 32

$V(D), 14$

$V_{D}, 14$

vértice acessa, 12, 15

acessível, 12, 15

emparelhado, 96

grau, 12

isolado, 12

novo, 10

vizinho, 11

vértices, 11, 14, 16

vetor, 10

componente, 10

incidência, 10

indexado por $V, 10$

integral, 10

suporte, 10

Vetta, 77

$V(G), 11$

$V_{G}, 11$

Wagner, 13, 81, 85, 89

Wakabayashi, 83

Weismantel, 104

Welsh, 9

Whitney, 14

Williams, 6, 65, 75, 76, 81, 84, 88

$w^{J}, 92$

$\overleftarrow{w}_{\min }(U), 83$

Woodall, 3, 7, 65, 69-73

Younger, 3, 8, 23, 27, 29, 32-35, 44-47, 49, $58,62,63,65,66,70,72,76,78,80$, 88,89

$\mathbb{Z}, 10$

$\mathbb{Z}_{\geq 0}, 10$

$\mathbf{0}, 17$

Zimmerman, 33 